\title{
Yaw Dynamics of Horizontal Axis Wind Turbines
}

\section{Final Report}

JUN 11 1992.

: COLORADO BOWOH.30\%

A.C. Hansen

University of Utah

Salt Lake City, Utah

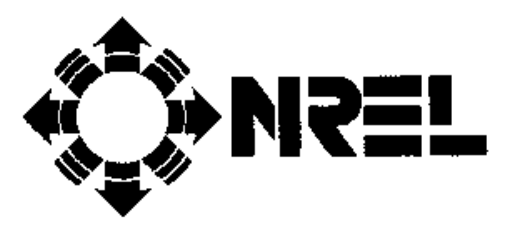

n 'tional Renewable Energy Laboratory Jivision of Midwest Research Institute erated for the U.S. Department of Energy der Contract No. DE-AC02-83CH10093 


\title{
Yaw Dynamics of Horizontal Axis Wind Turbines
}

\section{Final Report}

\author{
A.C. Hansen \\ University of Utah \\ Salt Lake City, Utah
}

NREL Technical Monitor: Alan Wright

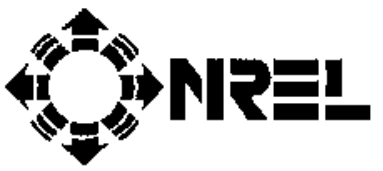

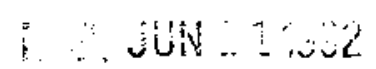

National Renewable Energy Laboratory (formerly the Solar Energy Research Institute) 1617 Cole Boulevard Golden, Colorado 80401-3393

A Division of Midwest Research Institute Operated for the U.S. Department of Energy under Contract No. DE-AC02-83CH10093

Prepared under subcontract no: XL-6-05078-2

May 1992 
On September 16, 1991 , the Solar Energy Research Institute was designated a natlonal laboratory, and its name was changed
to the National Renewable Energy Laboratory.

\section{NOTICE}

This report was prepared as an account of work sponsored by an agency of the United States government. Neither the United States government nor any agency thereof, nor any of their employees, makes any warranty, express or implied, or assumes any legal liability or responsibility for the accuracy, completeness, or usefulness of any information, apparatus, product, or process disclosed. or represents that its use wouid not infringe privately owned rights. Reference herein to any specific commercial product, process, or service by trade name, irademark, manuiacturer, or otherwise does not necessarily constitute or imply its endorsement, recommendation, or favoring by the United States government or any agency thereof. The views and opinions of authors expressed herein do not necessarily state or reffect those of the United States government or any agency thereof.

Printed in the United States of America

Available from:

National Technical Information Service

U.S. Department of Commerce

52.85 Port Royal Road

Springfield, VA 22161

Price: Microtiche A01

Printed Copy A09

Codes are used for pricing all publications. The code is determined by the number of pages in the publication. Information pertaining to the pricing codes can be found in the current issue of the following publications which are generally available in most libraries: Energy Research Abstracts (ERA); Govern. ment Reports Announcements and Index (GRA and I); Scientific and Technical Abstract Reports (STAR); and publication NTIS-PR-360 available from NTIS at the above address. 


\section{Notice}

This report was prepared as an account of work sponsored by the Solar Energy Research Institute, a Division of Midwest Research Institute, in support of its Contract No. DE-AC02-83-CH10093 with the United States Department of Energy. Neither the Solar Energy Research Institute, the United States Government, nor the United States Department of Energy, nor any of their employees, nor any of their contractors, subcontractors, or their employees, makes any warranty, express or implied, or assumes any legal liability or responsibility for the accuracy, completeness or usefulness of any information, apparatus, product or process disclosed, or represents that its use would not infringe privately owned rights. 
This Final Report is a summary of work that has been performed at the University of Utah over the past five years. Several graduate students contributed to the research: Xudong Cui, Norman Siedschlag, Robert Schnepp, and Todd Dorius each assisted in the analysis, programming, validating and debugging. Without their help this work would not have been possible. Papers, reports and theses which resulted from their efforts are listed at the end of the list of references.

Work of this type cannot be completed without the efforts and cooperation of many individuals. The SERI technical monitor, contract officials, test personnel and management have all approached this task with the goal of getting a job done in the most straightforward, cost-effective and reasonable manner. And many individuals at the University of Utah have shared that approach and attitude. That spirit is gratefully and warmly acknowledged.

Much of the material of Section 2.0 was originally commissioned by the Wind Turbine Company, Inc. Their permission to include this material in this report is gratefully acknowledged. 


\section{Table of Contents}

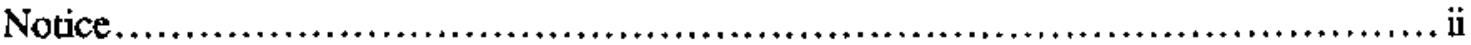

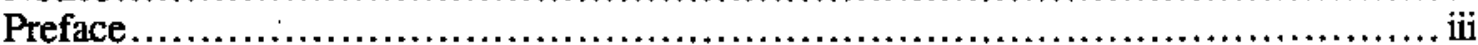

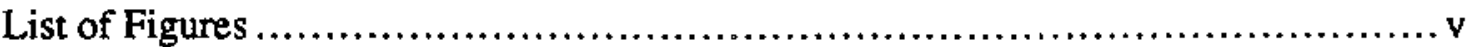

List of Tables ............................................................................ viii

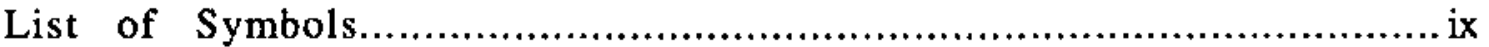

1.0 Introduction ....................................................................... 1

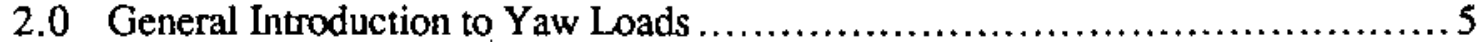

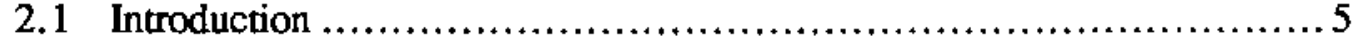

2.2 Hub forces and moments of a single blade ............................. 5

2.3 Hub forces and moments from multiple blades ......................... 7

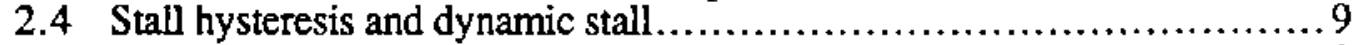

2.5 Wind disturbances which cause yaw loads............................ 9

2.6 Other causes of yaw loads ......................................... 15

2.7 Yaw dynamics of the teetering rotor........................................... 16

3.0 Theoretical Foundation................................................................ 17

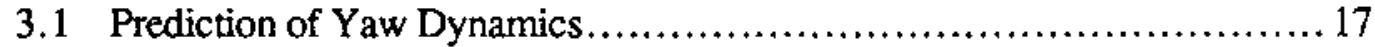

3.2 Relation between yaw and flap moments for a rigid rotor .................22

3.3 Equations of motion of the teetering rotor ............................ 24

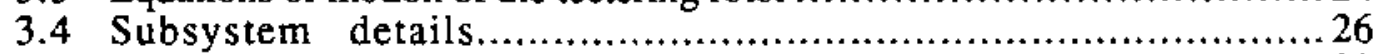

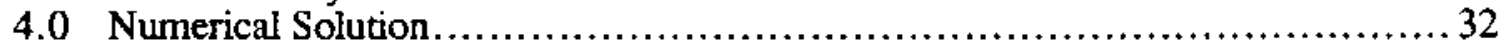

4.1 Numerical integration .............................................. 32

4.2 Initial conditions and trim solution ........................................ 32

4.3 Program structure and flow chart.......................................... 33

4.4 Computer requirements ............................................... 33

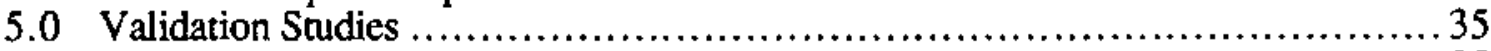

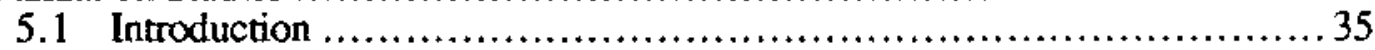

5.2 Mod-2 Wind Tunnel Test Comparisons .................................. 35

5.3 SERI Combined Experiment and FLAP Prediction Comparisons........... 38

5.4 Free-yaw predictions and measurements from the Combined Experiment

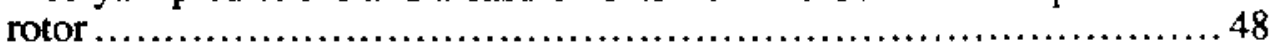

5.5 Teeter Predictions by YawDyn and STRAP...................................49

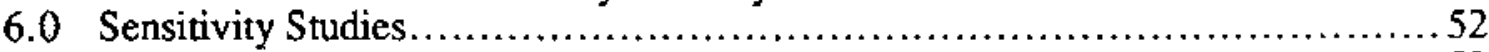

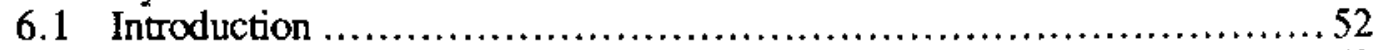

6.2 Rigid-hub configuration ............................................ 52

6.3 Teetering rotor configuration ...................................... 61

6.4 A Comparison of the Free-Yaw Behavior of Rigid and Teetering Rotors . . 64

7.0 Conclusions and Recommendations..............................................67

7.1 The YawDyn Model....................................................... 67

7.2 Yaw Loads on a Rigid Rotor ........................................ 68

7.3 Yaw Loads on a Teetering Rotor .......................................... 68

7.4 Yaw Motions of Rigid and Teetering Rotors..............................6 69

7.5 Recommendations for Additional Research ...........................69

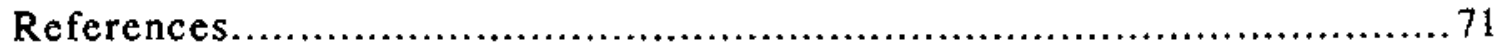

Appendix A Derivations of the Equations of Motion............................. A 1

Appendix B Characteristics of the Wind Turbines............................................ B1

Appendix C User's Guide to the YawDyn program......................................... 1 


\section{List of Figures}

Figure 2.1 Schematic view of the wind turbine showing the forces exerted

by one blade upon the hub.

Figure 2.2 Stall hysteresis for the SERI Combined Experiment calculated by

YawDyn. Wind speed $30 \mathrm{ft} / \mathrm{s}$, yaw angle $30^{\circ}$, no wind shear or

tower shadow.

Figure 2.3 Angle of attack of blades at two azimuth positions when the rotor operates with a yaw error.

Figure 2.4 Effect of vertical wind component on the angles of attack............... 13

Figure 2.5 Effect of horizontal wind shear on the angles of attack. ................ 14

Figure 3.1 Schematic of the rotor showing the primary blade variables. The view on the right is looking into the wind........................... 18

Figure 3.2 Hinged blade with torsional spring.

Figure 3.3 Comparison of the measured yaw moment (data points) with the moment calculated using the measured blade flap moment and the 1 p rotor mass imbalance.

Figure 3.4 Sketch of the teetering rotor, showing key parameters. Free teetering is permitted until the spring and damper are contacted by the hub (in the position shown).

Figure 3.5 Stall hysteresis loop measured at the $80 \%$ span on the Combined Experiment rotor.

Figure 3.6 Linear wind shear models. Horizontal shear in left sketch, vertical shear in right sketch. (Vertical shear can also be a powerlaw profile.)

Figure 4.1 Summary flow chart of the YawDyn computer program. 34

Figure 5.1 Comparison of predicted and measured mean yaw moments for the wind-tunnel model of the rigid-rotor Mod-2.

Figure 5.2 Comparison of predicted and measured $2 p$ yaw moments for the wind-tunnel model of the rigid-rotor Mod-2.

Figure 5.3 Comparison of YawDyn and FLAP predictions for a simple wind shear and tower shadow flow. All tower shadows have the same centerline deficit and the same width at the $75 \%$ span station.

Figure 5.4 Comparison of flap moments for the Combined Experiment data set $901-3$

Figure 5.5 Including dynamic stall in the YawDyn predictions makes a slight improvement in the accuracy of the cyclic loads. The data are the same as presented in Figure 5.4. 
Figure 5.6 Comparison of measured and predicted flap moments for

Combined Experiment data set $901-1$

Figure 5.7 An expanded view of Figure 5.6, concentrating on the first three seconds of the test.

Figure 5.8 Comparison of flap moments from Combined Experiment data set 901-2

Figure 5.9 Comparison of predicted and measured yaw moments. YawDyn predictions include a $180 \mathrm{ft}-1 \mathrm{~b}, 1 \mathrm{p}$ moment due to mass imbalance. Combined Experiment data set CE901-1.

Figure 5.10 Expanded view of Figure 5.9 showing the first three revolutions of the rotor. Combined Experiment data set CE901-1 45

Figure 5.11 Comparison of predicted and measured yaw moments. YawDyn predictions include a $180 \mathrm{ft}-1 \mathrm{~b}, 1 \mathrm{p}$ moment due to mass imbalance. Combined Experiment data set CE901-2.

Figure 5.12 Comparison of predicted and measured yaw moments. YawDyn predictions include a $180 \mathrm{ft}-1 \mathrm{~b}, 1 \mathrm{p}$ moment due to mass imbalance. Combined Experiment data set CE901-3.

Figure 5.13 Comparison of predicted and measured yaw moments showing the influence of stall hysteresis. YawDyn predictions include a $180 \mathrm{ft}-\mathrm{lb}, 1 \mathrm{p}$ moment due to mass imbalance. Combined Experiment data set CE901-3.

Figure 5.14 Angle of attack time history for data set 901-3. Note the characteristic $1 \mathrm{p}$ variation with a sharp dip caused by tower shadow

Figure 5.15 Comparison of stall hysteresis as measured at the $80 \%$ station and predicted at the $75 \%$ station for data set $901-3$.

Figure 5.16 Comparison of predicted and measured free-yaw response of the Combined Experiment rotor. At time $=0$ the rotor was released from rest at a yaw angle of $-32^{\circ}$. Data set number GS 144 .

Figure 5.17 Teeter amplitudes predicted by YawDyn and STRAP. 50

Figure 6.1 Variation of the predicted power output and mean flap moment of the Combined Experiment rotor. Wind speed $37 \mathrm{ft} / \mathrm{s}$, yaw angles from $+60^{\circ}$ to $-60^{\circ}$.

Figure 6.2 Variation of the predicted mean yaw moment of the Combined Experiment rotor. Wind speed $37 \mathrm{ft} / \mathrm{s}$, yaw angles from $+60^{\circ}$ to $60^{\circ}$

Figure 6.3 Variation of the predicted mean flap and yaw moments of the Combined Experiment rotor with hub-height wind speed. Yaw angle $0^{\circ}$ 
Figure 6.4 Variation of the predicted mean rotor power and angle of attack at the $75 \%$ station of the Combined Experiment rotor. Yaw angle $0^{\circ}$

Figure 6.5 Variation of angle of attack and axial induction factor at the 75\% station in the baseline configuration.

Figure 6.6 Yaw and root flap moments in the baseline configuration. 57

Figure 6.7 Stall hysteresis at the $75 \%$ station in the baseline configuration.

Figure 6.8 Yaw and flap moments with the rotor yawing against an effective stiffness of $4 \times 10^{5} \mathrm{ft}-\mathrm{lb} / \mathrm{rad}$. All other parameters match the baseline conditions of Table 6.1

Figure 6.8 Flap angle and angle of attack history for the baseline teetering rotor case. The teeter angle is the difference between the flap angle and the precone angle of $7^{\circ}$

Figure 6.9 Yaw and root flap moment for the baseline teetering rotor case

Figure 6.10 Free-yaw time history of the two rotors afrer release from rest at a $\pm 20^{\circ}$ yaw angle. Wind speed, $33.5 \mathrm{ft} / \mathrm{s}$; Vertical wind shear, 0.14 power law; Tower shadow, $10 \%$; Rigid- 80 blade stiffness, $2.5 \mathrm{p}$ (rotating)

Figure 6.11 Free-yaw time history of four rotors after release from rest at a $20^{\circ}$ yaw angle. Wind speed, $33.5 \mathrm{ft} / \mathrm{s}$; Vertical wind shear, 0.14 power law; Tower shadow, $10 \%$. 66

Figure 6.12 Effect of horizontal wind shear on the equilibrium yaw angle. Wind speed, $33.5 \mathrm{ft} / \mathrm{s}$; Vertical wind shear, 0.14 power law; Tower shadow, $10 \%$ 66 


\section{List of Tables}

Table 5.1 Parameters used in the analysis of the Mod-2 wind-tunnel model.

Table 5.2 Parameters used in the analysis of the Combined Experiment Rotor.

Table 5.3 Wind characteristics from the Combined Experiment data sets.

Values are Mean \pm Standard Deviation.

Table 5.4 Parameters used in the YawDyn and STRAP analyses of the

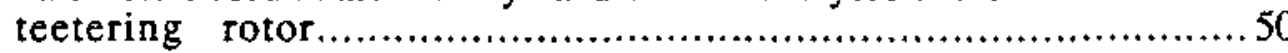

Table 6. I Baseline Conditions for the Rigid-Hub Sensitivity Studies.....................56

Table 6.2 Sensitivity of yaw moments to changes in machine and wind parameters

Table 6.3 Sensitivity of blade root flap moments to changes in machine and wind parameters.

Table 6.4 Baseline Conditions for the Teetered-Hub Sensitivity Studies 62

Table 6.5 Results of the teetered-hub sensitivity studies for the yaw moment. ESI-80 turbine operated at the conditions of Table 6.4, except as noted 


\section{List of Symbols}

$A=$ area

$a=$ axial induction factor $=\frac{V_{i}}{\bar{V}_{z}}$

$\mathrm{a}_{\mathrm{f}}=$ yaw friction moment

$a_{v}=$ coefficient to describe mechanical yaw damping system

$B=$ number of rotor blades

$\mathrm{C}_{\mathrm{L}}=$ section lift coefficient of blade airfoil

$C_{D}=$ drag coefficient of blade airfoil

$\mathrm{c}=$ blade chord length

$\mathrm{D}=$ drag force

$\mathrm{EI}=$ blade flap stiffness

$\hat{\mathrm{e}}_{\mathrm{x}}=$ unit vector in the $\mathrm{x}$ direction

$\mathrm{F}_{\mathrm{n}}=$ normal force component on blade element due to aerodynamic lift and drag

$\mathrm{F}_{\mathrm{t}}=$ tangential force component on blade element due to lift and drag

$\mathrm{g}=$ acceleration due to gravity, $32.174 \mathrm{ft} / \mathrm{sec}^{2}$

$\mathrm{h}=$ step size, in units of time, used to obtain numerical solution

$\mathrm{I}_{\mathrm{b}}=$ moment of inertia of blade about its flap axis

$\mathrm{I}_{\mathrm{L}}=$ moment of inertia of blade about its lag axis

$\mathrm{I}_{0}=$ moment of inertia of blade about its pitch axis

$I_{n}, I_{s}, I_{y}=$ moment of inertia of nacelle, rotor shaft and rotor hub about yaw axis

$I_{\text {yaw }}=$ total yaw moment of inertia less blade contribution, $I_{n}+I_{s}+I_{y}$

$\overline{\mathrm{I}}_{1}, \overline{\mathrm{I}}_{2}, \overline{\mathrm{I}}_{3}=$ principle mornents of inertia of the blade with respect to its mass center

$\mathrm{k}_{\mathrm{B}}=\mathrm{k}=$ blade hinge spring constant

$\mathrm{K}_{1}=$ constant used in the Gormont dynamic stall model, equations 3.17 and 3.18

$\mathbf{L}=$ lift force

$\mathrm{L}_{\mathrm{s}}=$ distance from rotor hub to yaw axis, approximately equivalent to the shaft length

$\mathbf{M}_{\mathrm{d} \gamma}=$ damping moment on yaw column

Medge=edgewise moment exerted by the blade upon the hub (by both blades for a teetering rotor)

$\mathrm{M}_{\text {flap }}$ = flapping moment on blade due to aerodynamic forces

$\mathrm{M}_{\text {hub }}=$ =moment exerted by teeter dampers and springs upon the teetering hub

$\mathrm{M}_{\text {yaw }}=$ yaw moment contribution from aerodynamic forces on blade

$\mathrm{M} \gamma=$ sum of aerodynamic, friction and damping moments about the yaw axis 
$\mathrm{m}_{\mathrm{b}}=$ blade mass (one blade)

$\mathrm{R}=$ rotor radius

$R_{h}=$ rotor hub radius

$\overline{\mathrm{R}}=$ distance from hinge to blade mass center

$\overrightarrow{\mathbf{r}}=$ position vector

$\mathbf{s}=$ teetered rotor undersling as shown in Figure 3.4

$\operatorname{Sign}(x)=$ Signum function $(+1$ if $x>0 ;-1$ if $x<0)$

$\mathrm{T}_{s}(\psi)=$ tower shadow function

$\mathrm{t}=$ time, blade thickness

$\overrightarrow{\mathrm{V}}=$ velocity vector

$\mathrm{V}_{\infty}=$ free stream wind velocity at hub height

$V_{i}=$ induced velocity due to air flow over blade

$\mathrm{V}_{\mathrm{n}}=$ normal component of relative velocity to blade

$V_{t}=$ tangential component of relative velocity to blade

$\bar{V}_{\mathrm{z}}=$ average axial wind speed, assumed equal to mean wind speed

$W=$ magnitude of the relative wind velocity with respect to the blade, $\sqrt{V_{n}^{2}+V_{l}^{2}}$

$\mathbf{X}, \mathbf{y}, \mathbf{z}=$ inertial reference frame (origin at tower top)

$X^{\prime}, Y^{\prime}, Z^{\prime}=$ yawed reference system (origin at tower top)

$\hat{X} \hat{Y} \hat{Z}=$ spinning reference system (origin at rotor center)

$\mathrm{x}, \mathrm{y}, \mathrm{z}=$ coned reference system (origin at blade hinge)

$\alpha=$ angle of attack

$\alpha_{b}=$ actual angle of attack used in the Gormont dynamic stall model, equation 3.17

$\alpha_{b} 0=$ zero-lift angle of attack

$\alpha_{m}=$ effective angle of attack used in the Gormont dynamic stall model

$\beta=$ flapping angle of blade

$\beta_{0}=$ precone angle of blade

$\Delta \mathrm{V}_{s}=$ tower shadow velocity deficit fraction

$\delta=$ wind direction angle

$\gamma=$ yaw angle

$\psi=$ blade azimuth angle, defined such that $\psi=0^{\circ}$ when blade is down (six o'clock position)

$\psi_{0}=$ half-angle of tower shadow region

$\Omega=$ blade rotation rate

$\theta=$ blade pitch angle, measured from chord line to plane of rotation

$\rho=$ air density, blade material density

$\tau=$ tilt angle of the low-speed shaft 
$\mathrm{T}=$ teeter angle

$\phi=$ inflow angle of relative velocity vector, $\tan ^{-1}\left(\frac{V_{n}}{V_{t}}\right)$

$\omega_{h}=$ natural flapping frequency of the hinged blade, $\sqrt{\frac{\mathrm{k}_{\beta}}{\mathrm{I}_{b}}}$

$\omega_{n}=$ ratio of blade natural flapping frequency to blade rotation frequency, $\sqrt{\frac{\omega_{h}^{2}}{\Omega^{2}}}$

Special Symbols

$\left({ }^{\circ}\right)$ = derivative with respect to time

()$^{\prime}=$ derivative with respect to azimuth angle $\psi$ 


\subsection{Introduction}

Wind turbines have been found cost-effective and efficient producers of electrical power. The increase in use of wind turbines has been phenomenal in the past several years. But progress and public and private support have been hindered by technical problems. One of the more serious problems has been yaw behavior of free-yaw rotors and yaw loads on controlled-yaw rotors. It has been reported that yaw-related problems are the second-leading cause of failures in the wind farms in California [Lynette, 1988].

\subsection{Background and Objectives}

Designers of a horizontal axis wind turbine yaw mechanism are faced with a difficult decision. They know that if they elect to use a yaw-controlled rotor then the system will suffer increased initial cost and increased inherent maintenance and reliability problems. On the other hand, if they elect to allow the rotor to freely yaw they know they will have to account for unknown and random, though bounded, yaw rates. They will have a higher-risk design to trade-off against the potential for cost savings and reliability improvement. The risk of a free-yaw system could be minimized if methods were available for analyzing and understanding yaw behavior. But the complexity of yaw behavior has, until recently, discouraged engineers from developing a complete yaw analysis method.

The history of wind energy development is rife with accounts of yaw related problems. The most serious problems are structural failures due to excessive yaw rates or yaw loads. This problem has been highlighted by failures of a number of rigid, three-blade systems in the Altamont and San Gorgonio Pass areas of California. Structural failures have also resulted from upwind operation of a nominal downwind rotor with a flexible blade root. In this situation thrust loads can drive the blades into the tower and cause major damage. Yaw-driven systems have had many instances of excessive yaw loads damaging the yaw drive mechanism. Apparently yaw loads are generally underpredicted by current design methods. Of lesser importance is loss of energy yield due to poor yaw tracking. Many free-yaw rotors consistently operate at small $\left(5^{\circ}-15^{\circ}\right)$ yaw errors and occasionally operate for extended periods at larger yaw angles. This problem can significantly impair energy yield and customer perception of the quality of the turbine.

With this motivation the following objectives were set for the current work:

Short term (first year): Provide a fundamental understanding of free-yaw mechanics and the design concepts most effective at eliminating yaw problems.

Longer term (five year): Provide tested design tools and guidelines for use by free-yaw wind system manufacturers.

The emphasis here is upon developing practical and sufficiently accurate design methods. It was desired that at the end of this research the wind turbine designer would be able to specify with confidence the yaw configuration needed to achieve benign and acceptable yaw behavior. The difficulty and complexity of the problem as well as the need to assist with near term development problems dictated the need for both a short - and long-term goal. The research described in this report has largely achieved both of these goals. A design model named YawDyn is now available for use in free-yaw and fixed-yaw analysis. It has been shown to be accurate in comparisons 
with test data and other analyses. However, the model relies upon empirical methods which are not proven for the wide range of rotors and wind conditions which can be conceived.

Yaw dynamics have been investigated by a number of researchers. The most comprehensive work was done by Swift [Swift, 1981]. He developed a model which was used as the starting point for the work reported herein. He used bladeelement/momentum aerodynamics and was the first wind turbine analyst to use the skewed wake effects as developed for helicopter analysis [Coleman, Feingold et al., 1945; Gaonkar and Peters, 1986; Pitt and Peters, 1981]. As will be shown later, these effects are crucial to correct estimation of aerodynamic yaw moments. He also considered induction lag, or the time delay between a change in thrust loading of a rotor and the induced velocity field of that rotor. His model uses linear aerodynamics and idealized twist and chord distributions to simplify the aerodynamics analysis.

de Vries [de Vries, 1985] discussed the inability of simple blade-element/momentum theory to adequately predict aerodynamic yaw moments on a rotor. He showed that a simple adjustment to the induced velocity field exhibited the correct qualitative influence on the moments. Swift, mentioned above, however was first to show a quantitative method based in physical principles for performing the adjustment.

Chaiyapinunt and Wilson [Chaiyapinunt and Wilson, 1983] showed that blade stiffness influences yaw motion by affecting the phase angle between an aerodynamic input such as tower shadow and the structural response. They showed that tower shadow will cause a steady yaw tracking error when rotor blades are not infinitely stiff. They also stated that blade element/momentum methods predict very small aerodynamic yaw. moments on a rotor unless the rotor has preconing.

Bundas and Dugundii [Bundas and Dugundji, 1981] performed wind tunnel tests and Miller [Miller, 1979] demonstrated theoretically that upwind rotors can be stable in the free-yaw upwind configuration. Such stable upwind operation of nominal downwind rotors has also been observed by the author and others in full-scale systems operating in the natural wind.

Stoddard [Stoddard, 1978; Stoddard, 1988] has developed analytical methods for examining the aerodynamics and stability of rotors. His methods linearize the equations of motion to achieve analytic solutions which are useful for examining dominant effects and trends in yaw behavior.

\subsection{Approach}

The work and experience just described set the requirements for the current model. It must be capable of the following:

1) The aerodynarnic model must include skewed wake effects and not rely solely upon blade-element/momentum methods.

2) Blade root flexibility must be considered in at least the flapping degree of freedom. This flexibility influences the response phase angle which in turn determines whether horizontal or vertical wind input asymmetry will cause a yaw response.

3) Both vertical and horizontal wind shear must be considered. Tower shadow must also be included. 
4) Response to time-rates-of-change in wind conditions must be determined. Changes in both wind speed and wind direction must be analyzed.

5) Use of the resulting methods as design tools strongly suggests calculations and outputs in the time domain. Theoretical operations in the frequency domain offer some advantages but are more difficult to understand and less likely to instill confidence in the user.

As the research progressed it was found that this list must be expanded to include the following:

6) Dynamic stall or stall hysteresis has a dramatic effect on yaw loads. Hysteresis in the airfoil characteristics contributes greatly to asymmetry which increases yaw loads.

7) A vertical component of the wind vector approaching the rotor will place a blade at the three o'clock or nine o'clock position in an advancing or retreating flow. This asymmetry will also contribute to yaw loads.

8) Similarly, tilt of the rotor axis will contribute to yaw loads. This results from the advancing and retreating blade loads and the component of the lowspeed shaft torque in the yaw direction.

9) Yaw-drive-train stiffness of a controlled yaw system can amplify or attenuate yaw loads depending upon the natural frequencies of the yaw drive and the rotor.

These requirements are imposed by the need to incorporate the essential physical mechanisms of the problem. Another requirement is imposed by the need to achieve practical, understandable and cost-effective solutions: The need for the simplest model possible. The philosophy of the development effort then has been to model each of the effects listed above in the simplest possible manner.

For example, the blade flexibility is modelled using an ideal root hinge/spring with a rigid blade. This permits the essential flap motion but avoids the details of modelling the actual mode shapes. As another example, the skewed wake induced velocity correction is a simple linear adjustment across the rotor disc. This adjustment permits the physical effect to be modelled without the confusion of higher order corrections. For a final example, the horizontal wind shear is modelled as linear shear. This is obviously an oversimplification of wind shear and large scale turbulence effects, but it permits investigation of the importance of wind shear.

Of course, a theoretical model cannot be depended upon until it has been thoroughly tested against turbine operating experience and other models. This is particularly true of models in the form of complex computer codes. It should not be surprising that more time has been devoted to testing the YawDyn code than to creating it. This report will present results from the comparison of prediction with wind-tunnel tests of a $1 / 20$ scale, rigid rotor, Mod-2 and the full-scale SERI Combined Experiment rotor. Such comparisons between actual turbine response and predicted response are the ultimate and necessary test of the physical validity of the model. However, the method of solution and details of the model algorithms are more readily tested through comparison amongst theoretical models. Thus some validation of the method has been accomplished via comparisons with the SERI FLAP code [Wright, Buhl et al., 1987; Wright, Thresher et al., 1991]. 
The remainder of this report is organized as follows: Section 2 will present a general discussion of the various sources of yaw loads. It is written as a qualitative description and avoids details and equations in the interest of providing an overview. Section 3 presents the governing equations of motion. The equations are derived in Appendices that can be skipped by the reader without loss of continuity. The governing equations are derived in their general form and then simplified to the forms which are available as options in YawDyn. Section 4 briefly discusses the numerical method of solution of the equations and the structure of the computer program. Section 5 presents results of sample calculations and comparisons with test data and other models for purposes of validation. Section 6 discusses the sensitivity of predicted yaw response to a variety of wind and turbine conditions. Section 7 provides conclusions and recommendations for additional research. Appendices also provide a listing and User's Guide to YawDyn as well as the equations of motion mentioned earlier. 


\subsection{General Introduction to Yaw Loads}

\subsection{Introduction}

This section is an introduction to the causes of wind turbine yaw loads and motions. It is intended to help the reader understand the fundamental mechanisms of yaw dynamics without becoming mired in the details and equations. The section concentrates on a qualitative discussion and is written for the engineer experienced in the analysis, design and terminology of wind turbine loads and dynamic response. Quantitative details are provided in later sections.

The focus of the discussion is the effect of the rotor on the yaw loads. Both rigid and teetering-hub rotors will be discussed. Though other factors may influence yaw, such as aerodynamic loads on the nacelle or a tail vane, or tower-top lateral vibration or tilt, they will not be discussed in this introduction. Emphasis is placed on the important factors influencing yaw. As mentioned, the discussion will be qualitative, with a minimum of equations or numerical results.

The discussion will begin with a description of how the blade root loads influence yaw loads. It will be seen that blade root flapping moments are the dominant cause of yaw loads for many wind turbines. Next a variety of wind conditions which can influence those root flapping moments will be described. Forces acting on the hub can also play a role in determining yaw loads. For a teetering rotor these forces are the dominant cause of yaw because the root flap moments have no load path into the rotor (or lowspeed) shaft. After a description of these hub forces, some inertial effects are described which can, in a mildly imbalanced system, be a very important source of undesirable yaw loads. Most of the above discussion will be for rigid hubs. A description will be given of the differences which result when a teetering rotor is used. These differences are very important. The report will conclude with the aforementioned references to where additional information can be found.

\subsection{Hub forces and moments of a single blade}

Figure 2.1 shows a simplified sketch of a downwind wind turbine with the blades removed. The forces and moments that one of the blades applies to the hub are shown. A coordinate system and two dimensions are also shown for use in the paragraphs that follow. The yaw moment is the moment about the tower longitudinal axis (parallel to the $\mathrm{X}$ axis). The sign convention in this report uses the right hand rule to define directions of positive moments and angles. Thus a positive yaw moment is one which would cause a clockwise rotation of the nacelle when viewed from above, looking down. The rotor rotates about the positive $\mathrm{Z}$ axis (clockwise when looking downwind).

The hub radius $\left(R_{h}\right)$, for purposes of this discussion, is the point at which the blade root flange is located. If there is no root flange it is the point at which the blade spar attaches to the hub, usually with some rapid change in the flapping stiffness. The yaw axis offset $\left(\mathrm{L}_{\mathrm{S}}\right)$ is the horizontal distance from the yaw axis to the vertex of the cone of revolution created by the undeflected, rotating blades (the center of the hub).

Concentrate for now on the effect of a single blade. Each of the three forces and the moment shown in Figure 2.1 can cause a yaw moment during some portion of a revolution of the blade. The flap moment (more properly called the out-of-plane bending moment for this discussion) has a component in the yaw direction except when 
the blade is vertical. Thus one contribution to the yaw moment will be the flap moment times the sine of the blade azimuth angle $\psi$ (defined such that azimuth is zero when the blade is pointed downward, in the six o'clock position). It is important to note this portion of the yaw moment is independent of the rotor offset from the yaw axis and of the hub radius.

The blade pitching and lead-lag root moments are generally unimportant when considering yaw loads. The blade pitching moment certainly contributes to the total yaw moment. But the magnitude of this moment is usually very small compared with other loads, and it is ignored. The lead-lag (or in-plane) moment will not contribute to the yaw moment if the rotor axis is horizontal. However, if the rotor axis is tilted (many wind turbines use a few degrees of tilt) then the lead-lag moment will contribute. In that case the vertical component of the low-speed shaft torque will be a contributor to the yaw moment.

Each of the three hub forces can be important sources of yaw loads. The out-of-plane (O-P) force can act through the hub radius moment arm to cause a yaw load. This yaw moment depends upon the horizontal component of hub radius as shown in the equation in Figure 2.1. The in-plane (I-P) force has a horizontal component which acts through the moment arm $\mathrm{L}_{\mathrm{S}}$ to create a yaw moment. Typically the moment arm for the I-P force is greater than that for the O-P force. Finally, the horizontal component of the tension force acts through the moment $\operatorname{arm} \mathrm{L}_{\mathrm{S}}$ to add a fourth term to the yaw moment equation. This blade root tension is generally a combination of centrifugal and gravity forces rather than aerodynamic forces. One can easily imagine that if the rotor had only one blade, and no counterbalance mass, the yaw moment caused by the mass imbalance would be extreme. Of course, with two or three blades the yaw moment will be a result of the imbalance in the masses and centers of gravity of the blades. This portion of the moment is directly proportional to $\mathrm{L}_{\mathrm{s}}$. Thus, if the designers wish to minimize the adverse effects of mass imbalance in the rotor, they should minimize $L_{S}$.

Recent research has shown that the flap moment term is the dominant source of yaw moments for well-balanced rigid rotor systems. In fact, it has been shown for at least two machines (a Howden 330 and the SERI Combined Experiment) that the yaw moment can be determined to a high degree of accuracy by simply performing vector addition of the contributions from the root flap moment of each of the three blades. This means that for these rotors the flap moment is so dominant that the yaw moment from the three hub forces can be neglected. This is quite remarkable, and in many ways fortunate (at least for the analyst). It means that the distance $\mathrm{L}_{\mathrm{S}}$ is not directly a factor in determining the yaw moment. This is counter-intuitive, but of great importance to the designer of the rigid rotor. 


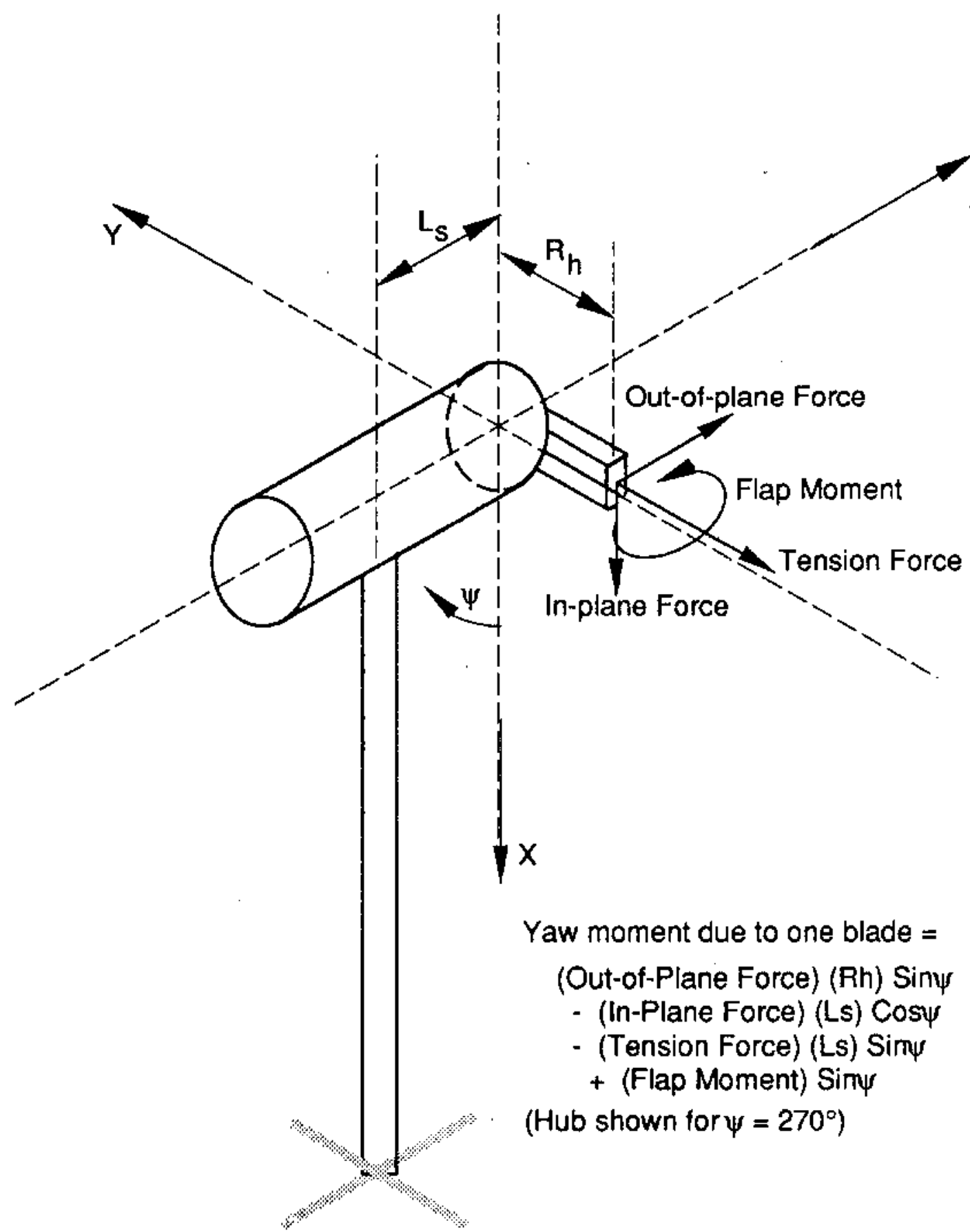

Figure 2.1. Schematic view of the wind turbine showing the forces exerted by one blade upon the hub.

\subsection{Hub forces and moments from multiple blades}

The system yaw loads are simply due to the contributions from each of the individual blade loads. This is easiest to visualize for a two-bladed rotor but is equally true for any number of blades. Imagine for a moment a perfectly balanced and matched set of blades on a two-bladed rotor. If the rotor axis is perfectly aligned with a perfectly uniform wind, then the loads on each of the blades will be identical and independent of blade azimuth. In this ideal case there will be no yaw moment. Any disturbance, whether aerodynamic or inertial, will upset this balance and cause some yaw load. 
When there is a disturbance the yaw loads will depend, generally in some complicated manner, upon differences in the loading on each of the blades. That is, the yaw loads depend upon differences in blade loads (or a vector sum) rather than an algebraic sum of the loads. Quite often the yaw loads result from small differences in large flap moments. On the SERI Combined Experiment rotor it is not uncommon to have mean flap moments of the order of $1000 \mathrm{ft}-1 \mathrm{bs}$ while the mean yaw moments are of the order of $100 \mathrm{ft}-1 \mathrm{bs}$. This complicates the understanding of yaw behavior because relatively small disturbances can create large differences in yaw loads.

Aerodynamic loads on blades of a high tip speed ratio rotor vary primarily as a result of variations in the angle of attack of the blade. Though the magnitude of the relative wind vector does change with changing wind conditions, most rotors operate such that the changes in angle of attack are more important to the loading than are changes in dynamic pressure. Thus to understand difference in aerodynamic loads on the two blades of a simple rotor it is necessary to look at the differences in angle of attack profiles along the two blades. The wide variety of wind disturbances which can cause yaw moments will be detailed in later sections. For now it is more useful to concentrate on the concept that differences in angle of attack on one blade compared with other blades will cause an imbalance of the forces and moments acting on the hub. This imbalance will in turn cause a yaw moment.

The time history of the disturbance will clearly affect the time history of the yaw load. If a disturbance repeats once per revolution of the blade (a frequency of 1p) then the result will be a steady (mean) yaw moment. For example, if every blade sees an increase in aerodynamic loading when it is at 9 o'clock and a decrease when it is at 3 o'clock then the disturbance to the blade will occur at $1 p$. But the system will see this disturbance as a steady load attempting to yaw the blade at 9 o'clock downwind. It is less clear intuitively but can easily be shown mathematically that the rotor will also see a $2 p$ disturbance. This is the well-known shift of $\pm 1 p$ as one transfers loads from the rotating (blade) reference frame to the stationary (nacelle) reference frame. That is, a $1 p$ disturbance on the blade is seen as a $0 \mathrm{p}$ (mean) and $2 \mathrm{p}$ disturbance at the yaw axis.

The number of blades on the rotor will determine which of the harmonics of an aerodynamic disturbance will be felt and which will be cancelled as loads are summed at the hub. An n-bladed rotor will experience mean yaw loads and cyclic yaw loads at. multiples of np. Thus a two-bladed rotor will have yaw loads at $0 \mathrm{p}, 2 \mathrm{p}, 4 \mathrm{p}, \ldots$ and a three-bladed rotor will have yaw loads at $0 \mathrm{p}, 3 \mathrm{p}, 6 \mathrm{p}, \ldots$ The $\mathrm{kp}$ yaw load harmonic will result from $(k+1) p$ and $(k-1) p$ blade loads. If a $3 p$ yaw moment hamonic is observed on the rotor it will have resulted from either $2 p$ or $4 p$ blade loads. The mean yaw moment is always caused by lp blade loads. If lp yaw moments are observed then there is a mass or pitch imbalance such that the blades are not all experiencing the same load history.

In the remaining discussion the examples will all use a two-bladed rotor. This is done for ease in visualizing the load summation and for direct applicability to the teetering rotor. However, all principles discussed using the two-bladed rotor examples will apply as well to the three-bladed rotor (with the appropriate changes in frequencies as noted above). The three-bladed rotor requires greater care in computing the vector summation of hub loads. But otherwise all of the discussion and operations are unchanged by the number of blades. 


\subsection{Stall hysteresis_and dynamic stall}

Stall hysteresis or dynamic stall has been shown to be very important in the determination of yaw behavior. Stall hysteresis is a dynamic effect which occurs on airfoils if the angle of attack of the airfoil changes more rapidly than the air flow around the blade can adjust. The result is auffoil lift and drag coefficients which depend not only on the instantaneous angle of attack (the usual quasi-steady aerodynamics assumption) but also on the recent angle of attack history. In particular, the lift and drag of the airfoil depend upon the angle of attack and the time rate of change of angle of attack.

Figure 2.2 shows estimated lift coefficients for the SERI Combined Experiment rotor when the rotor is yawed. The conditions for these calculations were: wind speed 30 $\mathrm{ft} / \mathrm{s}, 30^{\circ}$ yaw error, no wind shear or tower shadow. These values were generated using the YawDyn computer program, but test data show similar behavior. The top plot shows the characteristic 1p variation in angle of attack as the blade completes one revolution. The middle plot shows the lift coefficient with a large hysteresis loop which is typical of stall hysteresis or dynamic stall. The polar plot shows the $\mathrm{CL}$ variation vs. blade azimuth.

Though the dynamic stall phenomenon has long been known for helicopter rotors, it has been only recently that the existence and importance of dynamic stall has been demonstrated for wind turbines. Fortunately, we now have test data and simple theoretical models to help us understand unsteady aerodynamic effects.

The importance of dynamic stall to the yaw loads can be illustrated qualitatively with a simple comparison. Compare the lift coefficient of Figure 2.2 when the blade azimuth is between $180^{\circ}$ and $360^{\circ}$ with the $C_{L}$ when the blade is between $0^{\circ}$ and $180^{\circ}$. The average lift of the former is much greater than the latter. This imbalance of the lift from one side of the rotor to the other causes a yaw moment which is much larger than the load that would be seen if the hysteresis were not present in the lift coefficient. Without stall hysteresis the CL curve of Figure 2.2 would travel up and down along the top half of the loop shown. This shows that if the blade angle of attack varies around the revolution then the presence of stall hysteresis will exaggerate the imbalance of blade forces from one side of the rotor to the other. This enhanced imbalance will result in an increased yaw load over that which would be experienced without stall hysteresis. The reasons for cyclic variations in angle of attack will be discussed in the sections that follow.

\subsection{Wind disturbances which cause yaw loads}

Cross-wind (or yaw angle): The best known cause of yaw motion or loads is misalignment of the approaching mean wind vector with the rotor axis. With such a cross flow there will typically (though not always) be a yaw moment acting in an attempt to realign the rotor with the wind direction. Though the existence of such a yaw moment is intuitively apparent, it is less clear what the actual mechanism or cause of the moment might be. Figure 2.3 illustrates the situation and the mechanism which causes the yaw moment. With a cross-flow in the positive $Y$ direction a blade which is passing through the twelve $0^{\prime}$ clock $\left(\psi=180^{\circ}\right)$ position will be advancing into the crosswind. As shown in the figure, this will result in a decrease in the angle of attack at any given blade section. The blade will experience an increased angle of attack as it retreats from the crosswind when it is at the six o'clock position $\left(\psi=0^{\circ}\right)$. This situation is identical to that calculated in Figure 2.2. This means the blade experiences 
a once-per-revolution (lp) oscillation in angle of attack when the rotor operates at a yaw angle (in the absence of other disturbances).

Figure 2.2 shows that the average $C_{L}$ is highest for blade positions between $180^{\circ}$ and $360^{\circ}$. A blade which is not perfectly rigid will have a phase lag between the time the maximum aerodynamic load is applied and the time of maximum response (which is the time the blade root load is maximum). The amount of phase lag depends upon the stiffness and aerodynamic or mechanical damping of the blade. There is zero lag for an infinitely stiff blade and $90^{\circ} \mathrm{lag}$ for a very soft blade. This means the maximum root flap moment occurs for a blade angle between $180^{\circ}$ and $360^{\circ}$, just after the peak $\mathrm{CL}_{\mathrm{L}}$ is observed. At the time one blade sees the maximum root moment, the opposite blade sees the minimum moment. This is when the yaw moment due to the difference in root flap moments peaks.

The in-plane (edgewise) forces on the two blades also reach their maximum difference at this same blade angle. The net result is a yaw moment which would be in the negative $\mathrm{X}$ direction for the situation of Figure 2.2. If allowed to yaw under the influence of this yaw moment the rotor would tend to align with the wind direction.

Vertical wind or rotor tilt: A vertical wind through the rotor has much the same effect as a horizontal crosswind, with one important difference. Vertical wind causes the blade to be advancing or retreating when it is horizontal. This is the time at which an increased load will feed directly into a yaw moment, so long as the phase lag due to blade structural softness is not large. The concept is illustrated in Figure 2.4. This means that, all else equal on a rigid rotor, a vertical wind will create a higher yaw moment than a horizontal component of crosswind of the same magnitude. Fortunately, vertical winds tend to be much smaller than crosswinds. It would be unusual to have the approaching wind vector tilt $30^{\circ}$, while it is not uncommon at all to have a yaw error of the same magnitude.

Tilt of the rotor shaft has the same effect on the rotor aerodynamics as tilt of the approaching wind vector. Thus shaft tilt can be used to create a yaw moment beyond that mentioned earlier, namely the vertical component of the low-speed shaft torque.

Vertical shear of the approach wind: Vertical shear is the variation in approaching wind speed from the top to the bottom of the rotor. It is usually a result of the rotor being fully immersed in the planetary boundary layer, but vertical shear can also result from upwind obstructions such as other wind turbines or the wake of the tower of the rotor in question. Vertical shear causes a cyclic, $1 \mathrm{p}$, variation in angle of attack. In the typical case the wind at the top of the rotor, and hence the angle of attack and blade load, is higher than when the blade is pointed down. This imbalance of load from the top to the bottom of the rotor will not cause a large yaw moment unless there is a significant phase shift in the blade response (due to structural "softness").

Dynamic stall can increase the importance of vertical wind shear. If the wind speed is high enough that vertical shear causes the rotor to move in and out of stall, then the hysteresis in the CL curve can result in variations in blade root loads when the blades are horizontal. This is the fundamental requirement to generate an appreciable yaw load.

In summary, for rigid rotors vertical shear is not usually a major source of yaw loads. This is not the case for horizontal shear, the subject of the next section. 

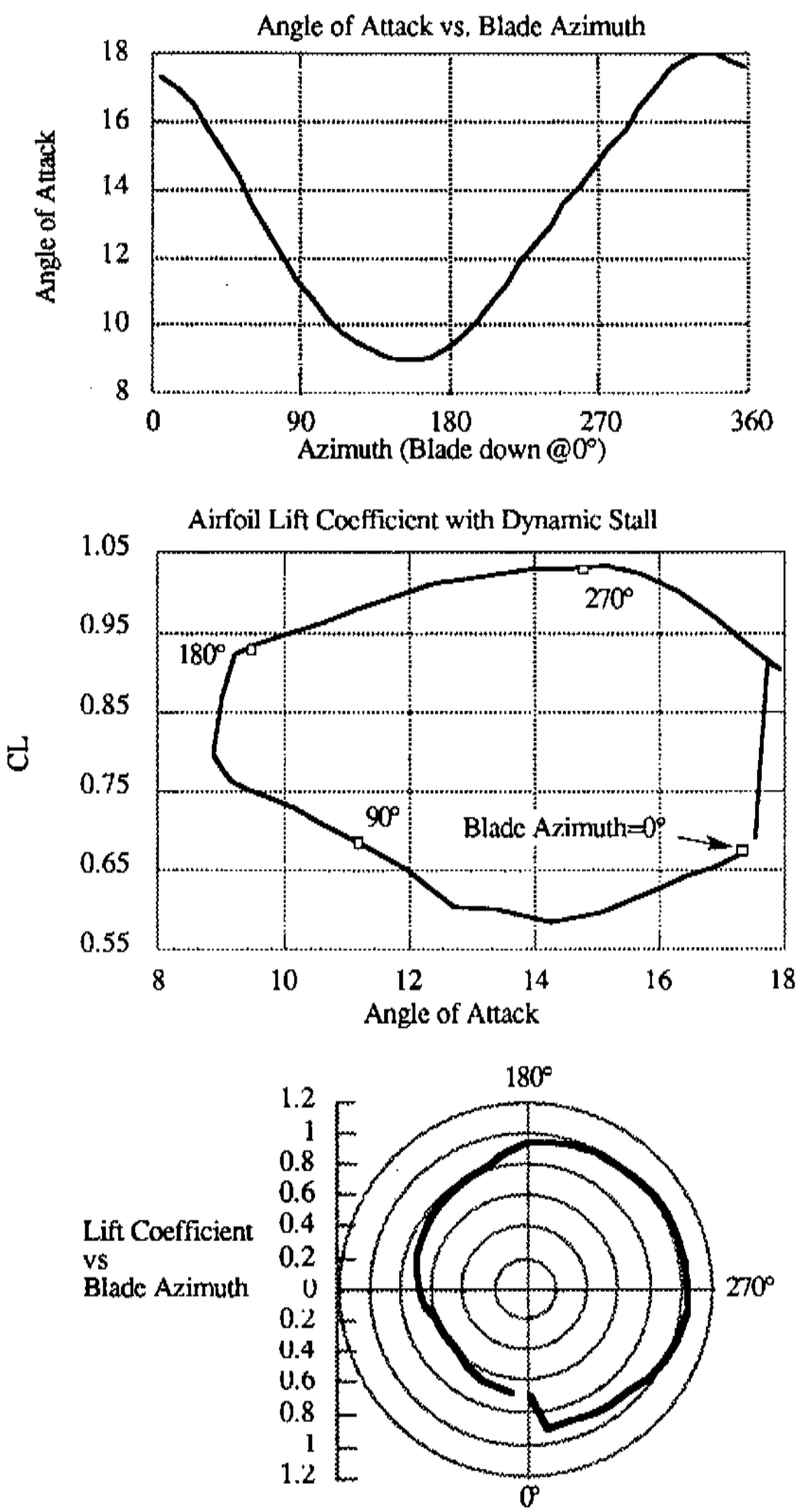

Figure 2.2. Stall hysteresis at the 75\% station of the SERI Combined Experiment as calculated by YawDyn. Wind speed $30 \mathrm{ft} / \mathrm{s}$, yaw angle $30^{\circ}$, no wind shear or tower shadow. 


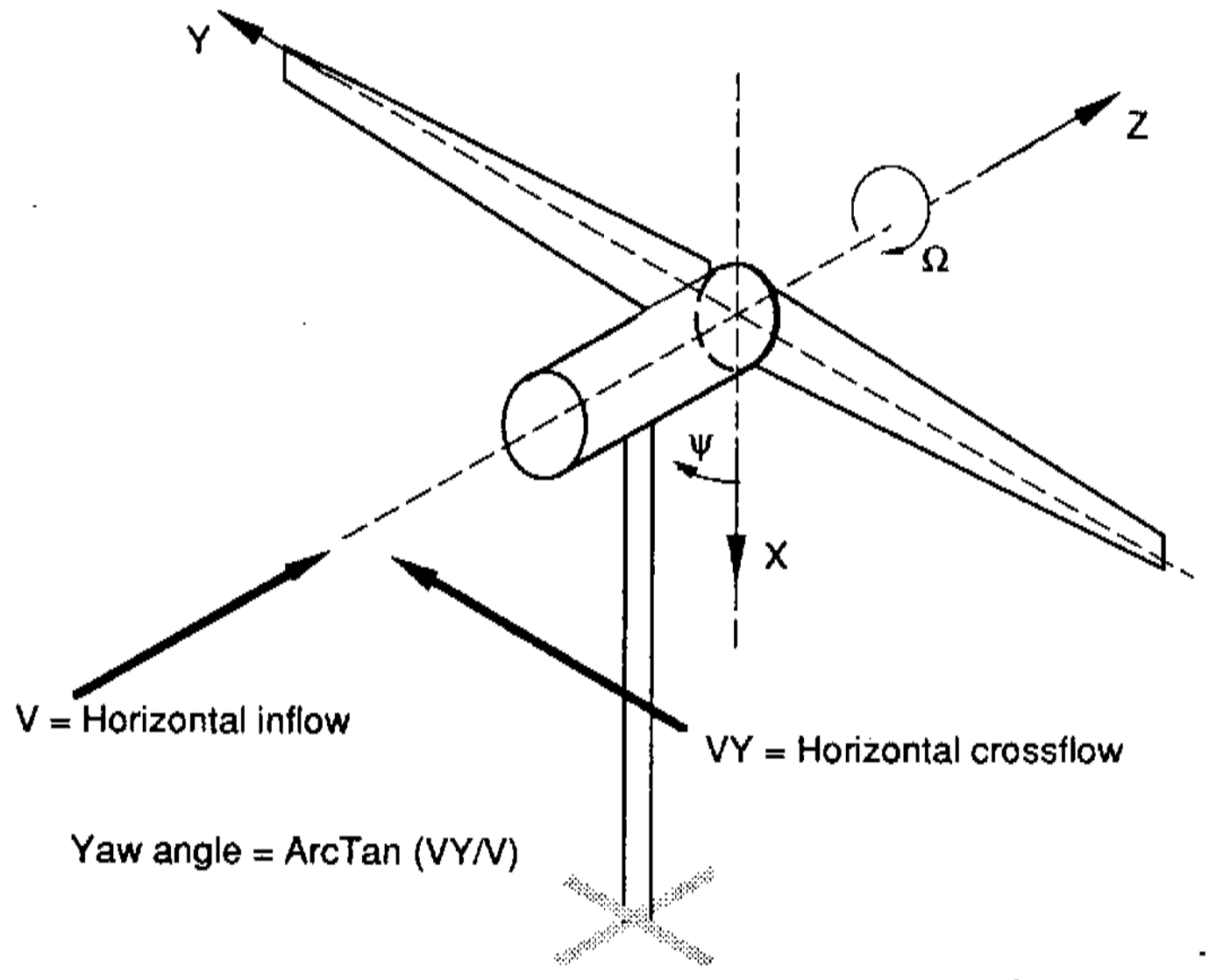

Advancing blade at $\psi=180^{\circ}$

Retreating blade at $\psi=0^{\circ}$
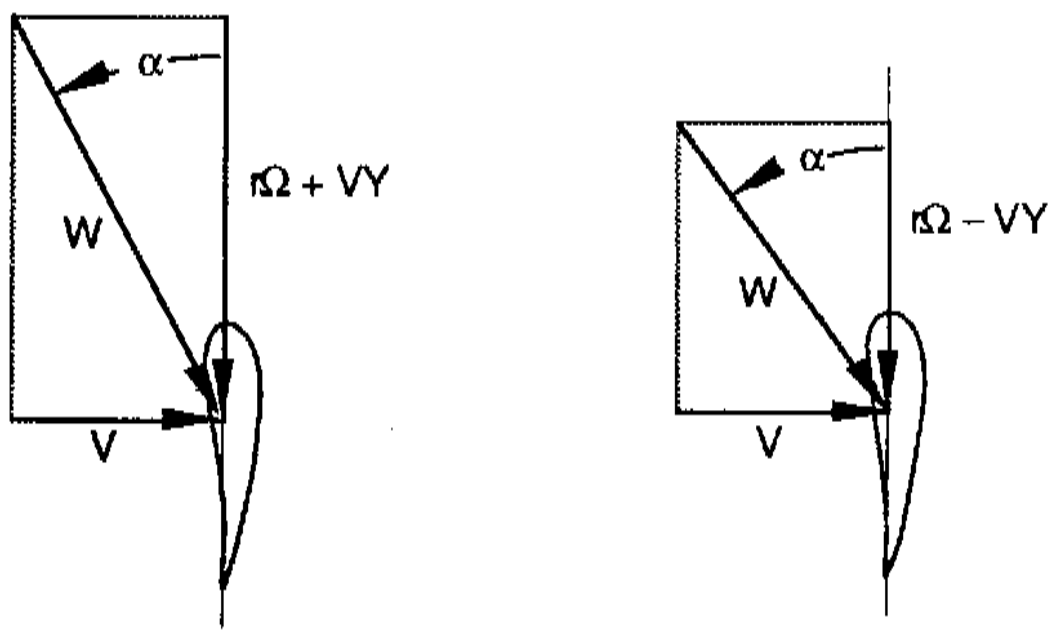

Positive horizontal crossflow (VY) decreases the angle of attack on the blade at $180^{\circ}$ azimuth. This decreased aerodynamic load causes little yaw moment unless there is a phase lag in the blade flap response (a sott blade) or stall effects such as stall hysteresis

Figure 2.3. Angle of attack of blades at two azimuth positions when the rotor operates with a yaw error. 


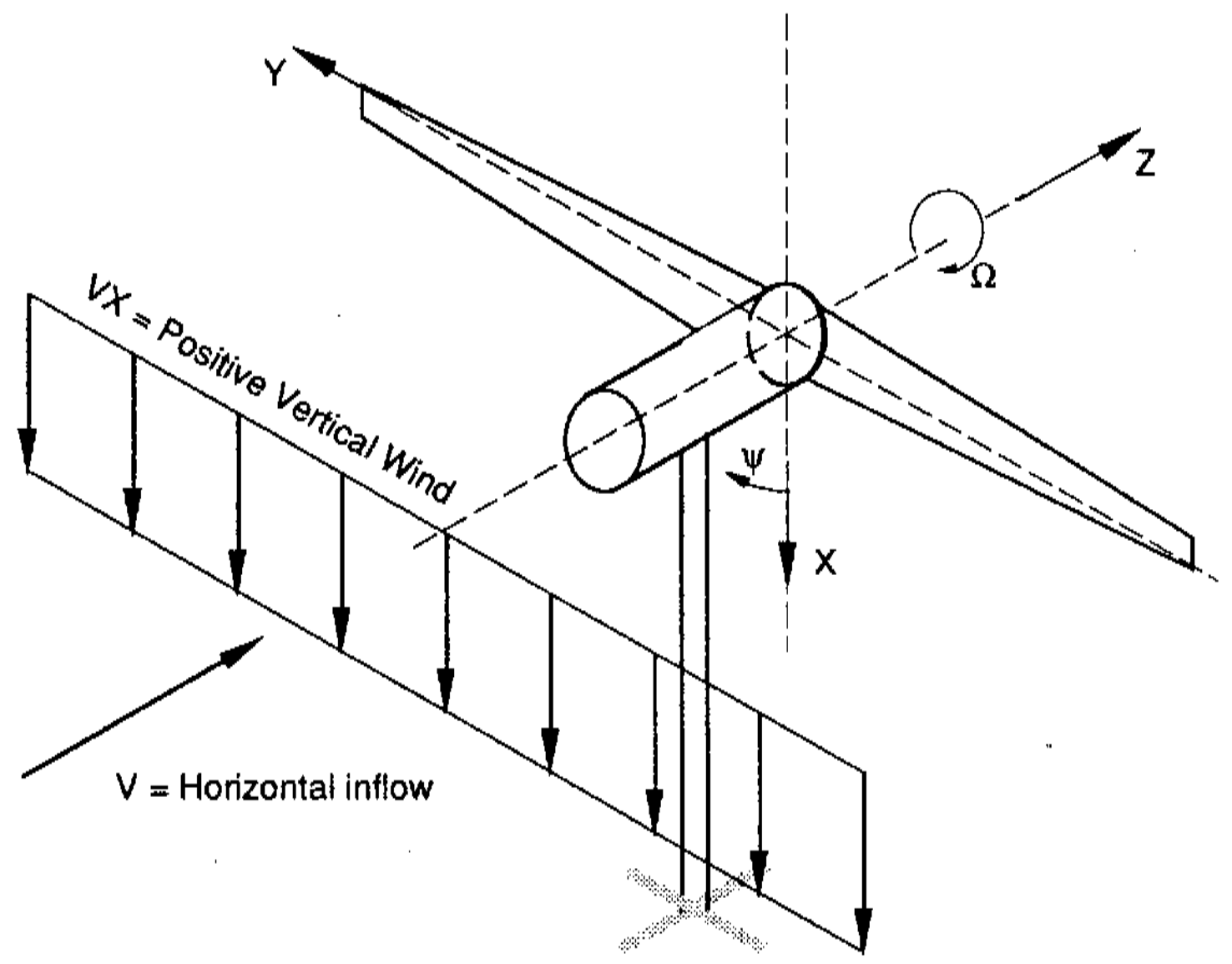

Advancing blade at $\psi=90^{\circ}$

Retreating blade at $\psi=270^{\circ}$
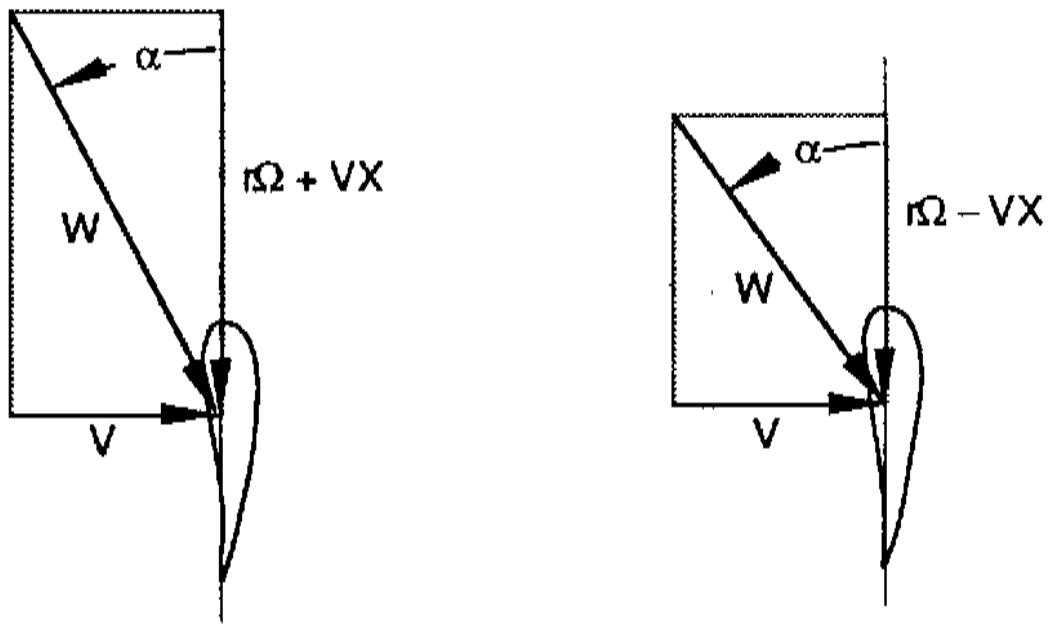

Positive (downward) vertical velocity decreases the angle of attack on the blade at $90^{\circ}$ azimuth. This decreased aerodynamic load causes a negative ( $\mathrm{X}$ direction) yaw moment

Figure 2.4. Effect of vertical wind component on the angles of attack. 


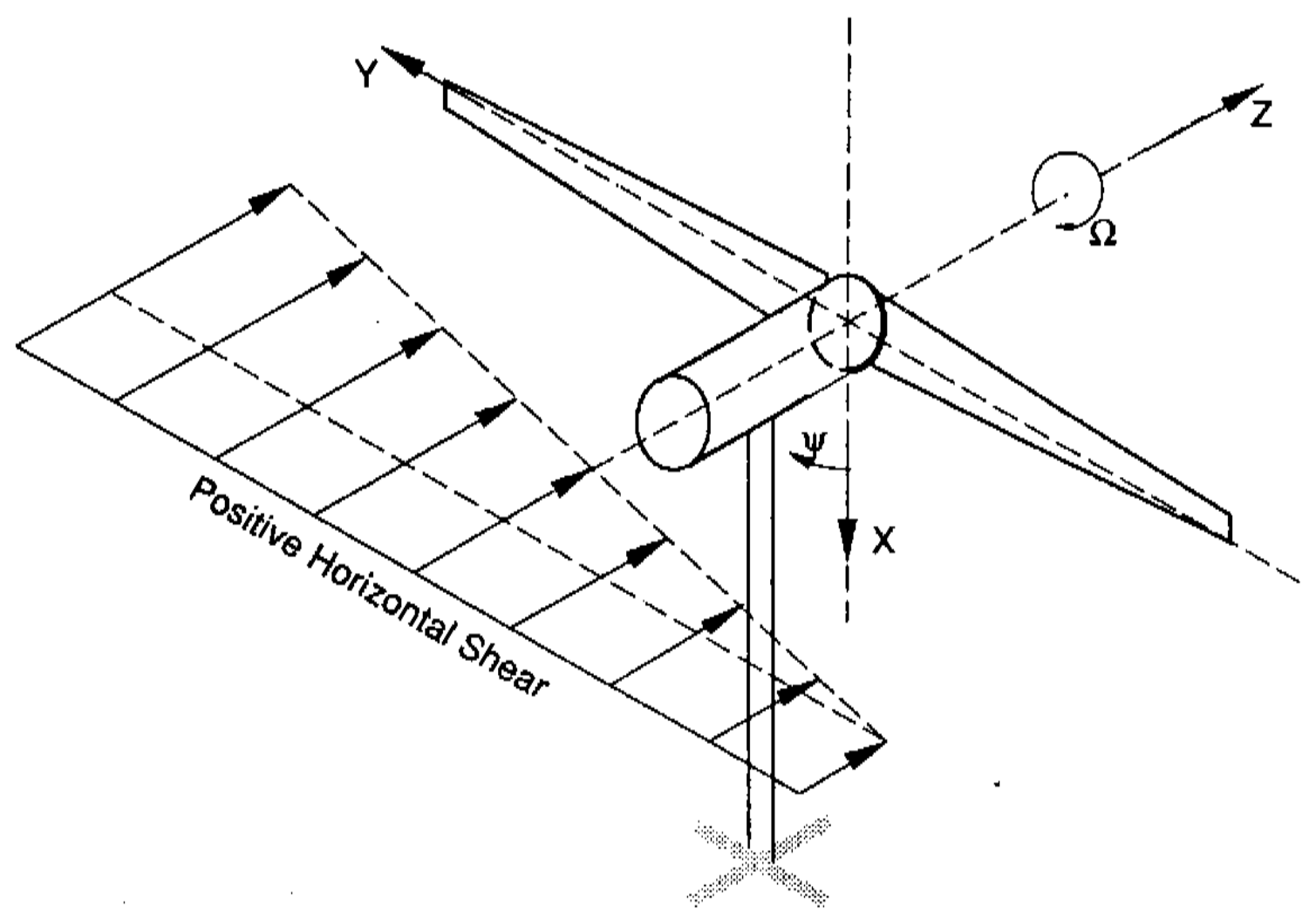

Blade at $\psi=90^{\circ}$

Blade at $\psi=270^{\circ}$
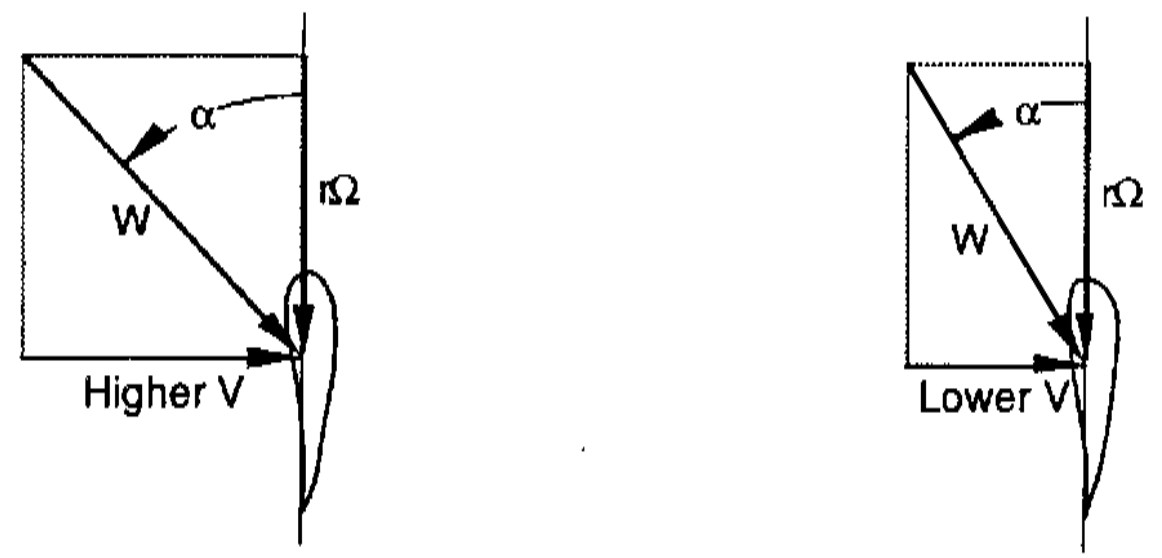

Positive horizontal shear increases the angle of attack on the blade at $90^{\circ}$ azimuth.

This increased aerodynamic load causes a positive (+ $X$ direction) yaw moment

Figure 2.5. Effect of horizontal wind shear on the angles of attack.

Horizontal shear of the approaching wind: Upwind obstructions such as other wind turbines or complex terrain can create strong, persistent variations in wind speed from one side of a rotor disc to the other. This horizontal shear of the wind speed also occurs in short, random periods as a result of turbulence. Horizontal shear has an effect much like vertical shear with one very important difference. Horizontal shear causes a maximum difference in blade flap loads when the blades are horizontal. Thus it acts when the blade is positioned to generate a yaw moment (if the rotor is rigid). This is illustrated in Figure 2.5. 
Measurements on a US Windpower rotor and the SERI Combined Experiment have shown that horizontal shear is usually the leading cause of yaw loads for these rigidrotor machines.

Turbulence: Turbulence is nothing more than a composite of all of the above wind conditions which is varying randomly in time. Thus at any instant the rotor is subjected to wind components in vertical, longitudinal and lateral directions. The wind speeds also vary across the rotor disk in an irregular manner. Turbulence changes the understanding of yaw mechanisms very little. Unfortunately, turbulence does add complexity to the interpretation of test data and the prediction of yaw behavior. These subjects are outside the scope of this introductory discussion and will not be pursued further.

\subsection{Other causes of yaw loads}

An ideal rotor is subjected to the sources of yaw loads discussed above. A real rotor is also subjected to other sources, and these sources can often dominate the cyclic yaw moment history of a rotor. Mass, center of gravity and pitch differences among the blades of a rotor are the sources of greatest interest.

Mass imbalance: If the blades of a rotor are not perfectly matched then centrifugal forces will cause a 1 p yaw moment. This can best be seen by picturing the effect of a mass imbalance as an offset of the c.g. of the rotor from the rotor axis. The centrifugal force at the mass center will be an in-plane force which rotates at the rotor speed. The resulting 1p yaw moment will be directly proportional to the mass offset, the distance $\mathrm{L}_{\mathrm{S}}$, and the square of the rotor speed.

Recent experiments at SERI showed that the mass imbalance was the the largest source of cyclic yaw moments on the Combined Experiment, even though considerable care was taken to add mass to the blades to achieve near balance. One organization in the San Gorgonio area has started offering a rotor balancing service. Apparently the rates of failures of the yaw drives have decreased on those systems which have been carefully mass balanced in the field. The message is clear: Great care should be taken in balancing the rotor in its operating configuration. Cyclic yaw loads are very sensitive to mass imbalance.

Pitch imbalance: If $1 \mathrm{p}$ yaw motion or loads are observed on a rotor which is carefully mass balanced, the likely cause is an aerodynamic imbalance. Differences in blade pitch or twist distribution are the common cause of aerodynamic imbalance. If one blade is set at a different pitch angle than the other, then a cyclic yaw load will be generated at the rotor speed. An aerodynamics analysis code such as PROP can be used to estimate the change in flap moment which is caused by an incremental change in pitch. This will be the amplitude of the cyclic yaw moment which results. Typically, blade pitch angles should be set to match within much less than one degree if aerodynamic imbalance is to be avoided.

Cyclic pitch: Another source of yaw loads which results from imperfect construction of the rotor is cyclic pitch. A rotor with pitch controls or a teetering rotor with pitch/flap coupling (delta-three) may have unplanned cyclic variations in blade pitch. If there is some play in the mechanical pitch linkage or torsional flexibility in the pitch control system, then cyclic variations in blade loads can cause variations in blade pitch. On the helicopter, cyclic blade pitch is used to control the direction of flight. The cyclic pitch causes a mean yaw moment (in wind turbine terminology) on the rotor which rotates the tip-path plane and hence the helicopter. The same thing can occur on the wind turbine if gravity or aerodynamic forces can cause changes in pitch angle. 
The teetering rotor with pitch/flap coupling may experience cyclic pitch resulting from teeter motion. This may be acceptable or even desirable in normal operation, but it can cause problems when a rotor is starting or stopping and there is little centrifugal stiffening or aerodynamic damping.

\subsection{Yaw dynamics of the teetering rotor}

The above discussion of yaw behavior has concentrated on the rigid rotor. When a teetering rotor is of interest there is a very important change in the behavior. Unless the teetering rotor strikes the teeter stops (or dampers and/or springs), it has no load path to carry the blade root flap moments into the low-speed shaft or the yaw system. Recall that the root flap loads are the dominant cause of yaw loads of the rigid rotor. Thus, the dominant cause of yaw has been eliminated by the teetering rotor. This means that yaw loads and/or yaw rates will generally be lower for the teetering rotor. Of course, a teeter stop impact can transmit a large bending moment into the shaft and this in tum will cause a large yaw load if the blade is not vertical at the time of impact.

If root flap moments can no longer be important to the yaw behavior, then the rotor forces will become the dominant cause of yaw. Rotor forces fluctuate as a result of changes in angle of attack as discussed at length above. To interpret the effect of a given wind input, evaluate its effect on the angle of attack (and blade force) and determine where in its rotation the blade will be most affected. Thus the basic discussion is still applicable, but one must exercise care in interpreting the relative importance of the different inputs to the rotor. For example, the distance $\mathrm{L}_{\mathrm{S}}$ will become more important, because yaw moments will be directly proportional to the hubyaw axis offset. This is quite different from the situation where flap moments dominate and make $\mathrm{L}_{\mathrm{S}}$ relatively unimportant.

The sections that follow in this report quantify this discussion and present a computer model which makes it possible to explore these phenomena in detail for a variety of rotor configurations. 


\subsection{Theoretical Foundation}

\subsection{Prediction of Yaw Dynamics}

Modeling of yaw dynamics is complicated by the variety of machine and wind characteristics which are important to yaw response. The goal of the current modeling is to develop the simplest model possible while not neglecting significant effects. Previous research indicated the following features must be included in the model;

- Blade root flexibility and flap motion

- Aerodynamic model must include stall effects

- Correction for effects of skewed wake aerodynamics on the induced velocities

- Vertical and horizontal wind shear and tower shadow

- Mechanical yaw damping and friction

- Arbitrarily large yaw angles but small flap angles

- Both free- and fixed-yaw behavior

During the course of this research additional factors were found to be important:

- Dynamic stall

- Vertical component of wind speed

- Stiffness of the yaw restraint of a "fixed" yaw rotor

- Tilt of the low speed shaft

These effects and features are implemented in the current models. The result is a set of equations for yaw and flap motion in the time domain. Simple blade element/momentum aerodynamics are used but corrections are made that account for the interdependence of induced velocities at neighboring blade elements when the wake is skewed with respect to the rotor. Two-dimensional airfoil tables are used to represent the blade lift and drag coefficients and the NASA synthesization method is used to obtain static airfoil characteristics in deep stall [Viterna and Corrigan, 1981]. The Gormont model is used to represent stall hysteresis. The contributions to the yaw moment of all blade flap and lead-lag forces and moments are included in the calculations. Thus the " $\mathrm{H}$ " force of helicopter terminology is included (though the leadlag vibratory degree of freedom is not considered).

Some details of the model are presented in the paragraphs that follow. Appendix A contains a derivation and discussion of the equations of motion which are presented below. The algebraic manipulations required for the derivations were all performed using the Mathematica $@$ symbolic manipulation program [Wolfram, 1991]. Appendix A contains the Mathematica files used in the derivations.

\section{HAWT Representation}

A simplified model for a two blade HAWT is shown schematically in Figure 3.1. Only yaw motion, $\gamma$, and blade flapping motion, $\beta$, have been used in the development of the equations of motion. A teetering degree-of-freedom can be substituted for the flap motion. When teetering is modeled, the blade is completely rigid. Additional degrees of freedom, such as blade pitch and lag motion, are not considered to be as important to yaw response and have been ignored. 


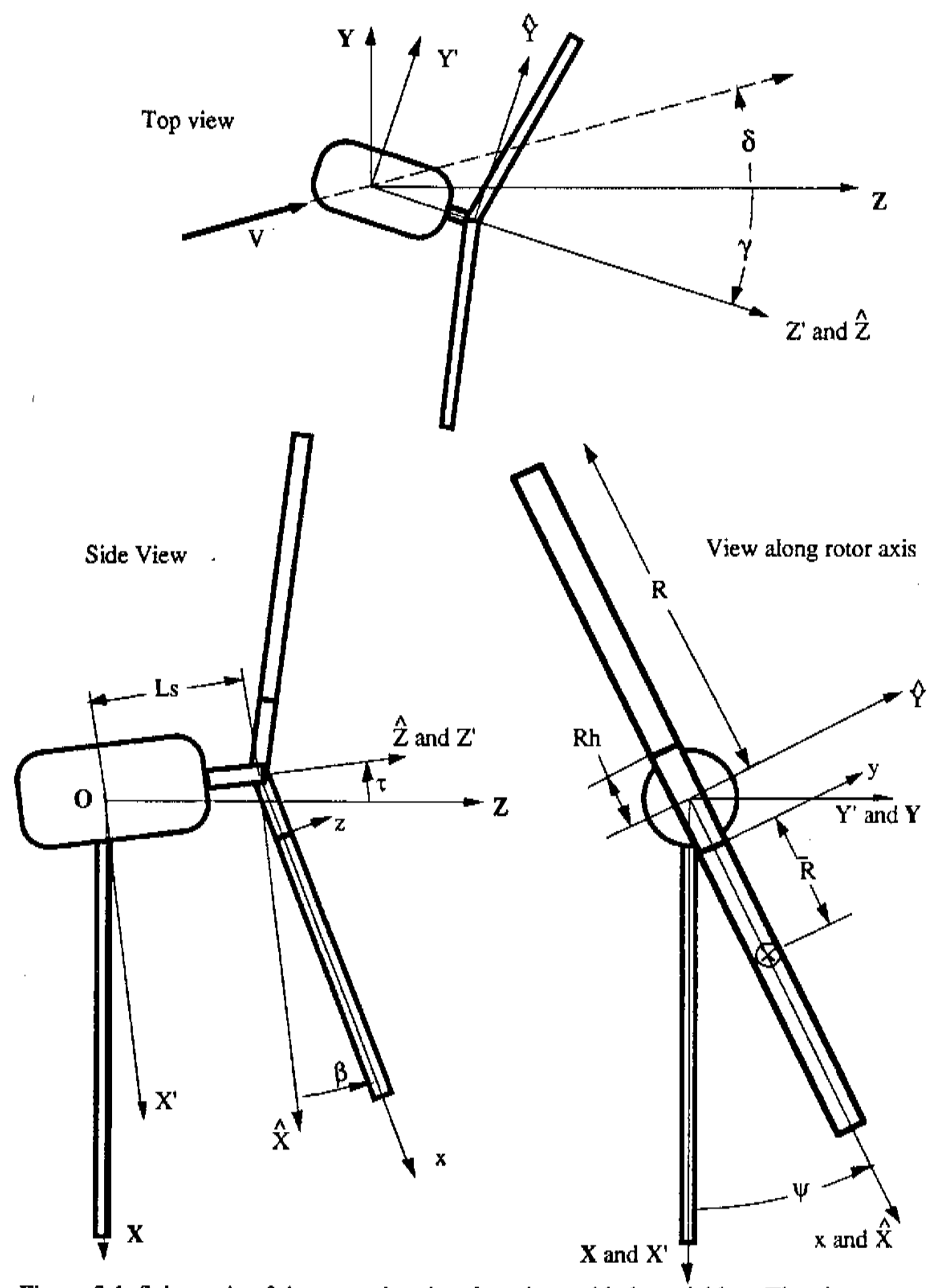

Figure 3.1 Schematic of the rotor showing the primary blade variables. The view on the right is looking into the wind. 


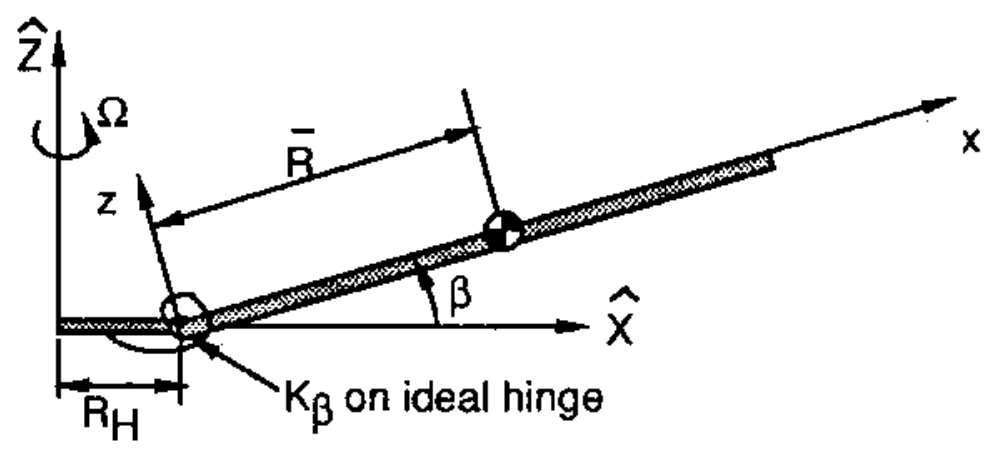

Figure 3.2 Hinged blade with torsional spring

A rigid tower (no top deflection or rotation) is used in the model. This permits the inertial reference frame $X Y Z$ to be located with its origin (point $O$ in Figure 3.1) at the intersection of the yaw column and the rotor shaft. $X^{\prime} Y^{\prime} Z^{\prime}, \hat{X} \hat{Y} \hat{Z}$ and $x y z$ designate the nacelle, rotor (hub) and blade reference frames used in developing the equations of motion. The distance from yaw axis to the rotor, the approximate shaft length, is designated by $\mathrm{L}_{\mathrm{s}}$.

The rotor shaft is tilted at angle $\tau$ above the horizontal. (All angles in Figure 3.1 are shown in their positive sense.) The flap angle $\beta$ is measured with respect to the plane perpendicular to the axis of rotation. When the rotor is teetered then the flap angles of the two blades are related by the simple equation:

$$
\beta_{2}=2 \beta_{0}-\beta_{1}
$$

Here $\beta_{0}$ is the precone angle and $\beta_{i}$ is the flap angle of blade $i$.

The rotor rotation rate, $\Omega$, is considered to be constant for each specified operating condition. The blade azimuth position, $\psi$, is defined with respect to the six o'clock position of the rotor disk. This azimuth angle will be used to describe the positions of all the rotor blades.

The nacelle, rotor shaft and blades are treated as rigid bodies. The blades are connected to the rotor hub by frictionless hinges which permit only out-of-plane flapping motion. Torsional springs with stiffness $\mathrm{k}_{\beta}$ are attached as shown in Figure 3.2. This models the elastic deflections of each wind turbine blade. Typically the spring stiffness, $\mathrm{k}_{\beta}$, is selected to match the flap natural frequency to the first flap bending mode of the actual blade.

The blade flap angle is a function of the instantaneous wind velocity as seen by the blade as it rotates about the shaft. This will cause the $\mathrm{i}^{\text {th }}$ blade to assume its own motion, described by $\beta_{\mathrm{i}}$, independent of each other blade. This gives the model $\mathrm{B}+1$ degrees of freedom where $B$ is the number of blades. There is one exception to this general statement. If the teetering rotor is modeled then the blades do not flap independently and the model has only one flap degree of freedom and the yaw degree of freedom. 
The governing equations were derived from Euler's equation and the more general equations of three-dimensional, rigid body motion. The resulting equation for the flap motion is:

$$
\begin{aligned}
\beta^{\prime \prime}= & -\beta-\frac{k}{I_{b} \Omega^{2}}\left(\beta-\beta_{0}\right)+\frac{M_{f l a p}}{I_{b} \Omega^{2}}-\beta \frac{m_{b} \bar{R} R_{h}}{I_{b}}-\frac{m_{b} g \bar{R}}{I_{b} \Omega^{2}}(\tau+\beta \cos \psi) \\
& -2 \gamma^{\prime} \cos \psi\left[1+\frac{m_{b} \bar{R} R_{h}}{I_{b}}\right] \\
& +\gamma^{\prime}=\left[\frac{m_{b} L_{s} \bar{R}}{I_{b}}+\tau \cos \psi\left(1+\frac{m_{b} \bar{R} R_{h}}{I_{b}}\right)+\beta\left(\cos ^{2} \psi-\frac{m_{b} \bar{R} R_{h}}{I_{b}} \sin ^{2} \psi\right)\right] \\
& -\gamma^{\prime \prime} \sin \psi\left[1+\frac{m_{b} \bar{R} R_{h}}{I_{b}}+\frac{m_{b} L_{s} \bar{R}}{I_{b}} \beta\right]
\end{aligned}
$$


The equation for yaw angle is (when the flap degree of freedom is included) is:

$$
\begin{aligned}
& \gamma^{\prime \prime}\left[\begin{array}{l}
I_{y a w}+B m_{b} L_{s}^{2}+m_{b} \bar{R} L_{s} \sum_{i=1}^{B} \beta_{i}\left(1+\cos ^{2} \psi_{i}\right) \\
-\frac{m_{b}^{2} \bar{R}^{2} R_{h}}{I_{b}}\left(L_{s} \sum_{i=1}^{B} \beta_{i} \sin ^{2} \psi_{i}+R_{h} \sum_{i=1}^{B} \sin ^{2} \psi_{i}\right)+m_{b} R_{h}^{2} \sum_{i=1}^{B} \sin ^{2} \psi_{i}
\end{array}\right]= \\
& -\frac{1}{\Omega^{2}} \sum_{i=1}^{B} M_{e^{d g e_{i}}}\left(\tau+\beta_{i} \cos \psi_{i}\right)+\frac{m_{b} \bar{R} R_{h}}{I_{b} \Omega^{2}} \sum_{i=1}^{B} M_{\operatorname{lap}_{i}} \sin \psi_{i} \\
& +\frac{1}{\Omega^{2}} \sum_{i=1}^{B}\left(L_{s} \beta_{i}+R_{h}\right) F_{N_{i}} \sin \psi_{i}-\frac{1}{\Omega^{2}} \sum_{i=1}^{B}\left(L_{s} F_{T_{i}} \cos \psi_{i}+R_{h} \tau F_{T_{i}}\right) \\
& +\left(m_{b} \bar{R} R_{h}+\frac{k}{\Omega^{2}}\right)\left(1+\frac{m_{b} \bar{R} R_{h}}{I_{b}}\right) \sum_{i=1}^{B} \beta_{i} \sin \psi_{i} \\
& +\frac{m_{b} g \bar{R}}{\Omega^{2}}\left(1+\frac{m_{b} \bar{R} R_{h}}{I_{b}}\right) \sum_{i=1}^{B} \beta_{i} \cos \psi_{i} \sin \psi_{i} \\
& -2 \gamma^{\prime}\left[m_{b} R_{h}^{2}\left(1-\frac{m_{b} \bar{R}^{2}}{\Omega^{2}}\right) \sum_{i=1}^{B} \cos \psi_{i} \sin \psi_{i}+m_{b} L_{s} \bar{R} \sum_{i=1}^{B} \beta_{i}^{\prime}\right] \\
& +\gamma^{\prime 2}\left[\begin{array}{l}
\tau\left(-I_{b}-m_{b} \bar{R} R_{h}+m_{b} R_{h}^{2}-\frac{m_{b}^{2} \bar{R}^{2} R_{h}^{2}}{I_{b}}\right) \sum_{i=1}^{B} \cos \psi_{i} \sin \psi_{i} \\
-m_{b} \bar{R} R_{h} \sum_{i=1}^{B} \beta_{i} \sin \psi_{i}-I_{b} \sum_{i=1}^{B} \beta_{i} \cos ^{2} \psi_{i} \sin \psi_{i} \\
-m_{b} \bar{R} R_{h} \tau \sum_{i=1}^{B} \cos \psi_{i} \sin \psi_{i}+\frac{m_{b}^{2} \bar{R}^{2} R_{h}^{2}}{I_{b}} \sum_{i=1}^{B} \beta_{i} \sin ^{3} \psi_{i} \\
-I_{b} \sum_{i=1}^{B} \beta_{i} \cos ^{2} \psi_{i} \sin \psi_{i}
\end{array}\right]
\end{aligned}
$$

For a rigid rotor $\left(\beta_{i}^{\prime}=\beta_{i}{ }^{\prime \prime}=0, \beta_{i}=\beta_{0}\right)$, the HAWT model possesses only a single (yaw) degree-of-freedom with the equation of motion becoming:

$$
\begin{array}{r}
\gamma^{\prime \prime}\left[I_{\text {yaw }}+B m_{b}\left(L_{s}^{2}+2 \bar{R} L_{s} \beta_{0}\right)+\left(I_{b}+\right.\right. \\
\left.\left.m_{b} R_{h}^{2}+2 m_{b} \bar{R} R_{h}\right) \sum_{i=1}^{B} \sin ^{2} \psi_{i}\right] \\
+\frac{a_{y}}{\Omega} \gamma^{\prime}+\frac{a_{f}}{\Omega^{2}} \operatorname{sgn}\left(\gamma^{\prime}\right)=\frac{M_{\text {yaw }}}{\Omega^{2}}
\end{array}
$$

In this equation $\mathrm{M}_{\mathrm{yaw}}$ is the net aerodynamic yaw moment acting on the rotor. 
Equations 3.2 and 3.3 are the complete set of equations implemented in the program YawDyn for the rigid hub rotor. Equation 3.4 is the governing equation solved when the blade flap DOF is ignored. This equation can be solved more quickly and can be used for comparison with other rigid blade analyses.

Readers who are familiar with annual reports from this project may notice the yaw equation (with flap) differs from that published earlier. There was an error in the earlier derivation using Lagrange's method that was discovered only recently. The previous equation from the earlier reports should be ignored, as should earlier releases of YawDyn implementing that equation. The error affected only those results with coupled free-yaw and flap motion. This was a small fraction of the earlier work published. All results pertaining to fixed yaw and yaw motion without flap are believed to be correct.

\section{Relation between yaw and flap moments for a rigid rotor}

Yaw loads on a wind turbine result from aerodynamic and dynamic forces on the rotor and the nacelle. The current analysis considers only the rotor loads and neglects the nacelle aerodynamic moment. The author considers this acceptable since the nacelle area is small in comparison with the rotor area and the aerodynamic yaw moments will likewise be small unless a tail or other yaw vane has been added to the nacelle. The loads on the rotor can be divided into two categories of interest in yaw behavior: 1) the net horizontal force on the hub, often called the $\mathrm{H}$-force in helicopter analysis and 2) the summation of the blade flap moments, resolved in the yaw direction. Shaft tilt, which provides a component of low-speed shaft torque in the yaw direction, is also. considered in the present model.

Consider a three-blade, rigid rotor wind turbine operating in steady-state conditions. In this situation all blades will experience the same flap moments, each lagging the previous by $120 \mathrm{deg}$. The flap moment for a single blade can be expressed in a Fourier series as follows:

$$
M_{f}=\sum_{n=0}^{\infty}\left[f_{n_{c}} \cos (n \psi)+f_{n_{s}} \sin (n \psi)\right]
$$

In this equation the coefficients $f_{n}$ are constants which represent the sine and cosine components of each harmonic. $\psi$ is the blade azimuth angle, equal to 0 deg when the blade is at the six o'clock position. The yaw moment is the sum of the three flap moments resolved in the yaw direction:

$$
\mathrm{M}_{\gamma}(\psi)=\sum_{\mathrm{i}=0}^{2} \mathrm{M}_{\mathrm{f}}\left(\psi+\mathrm{i} \frac{2}{3} \pi\right) \sin \left(\psi+\mathrm{i} \frac{2}{3} \pi\right)
$$

This yaw moment can also be expressed in terms of a Fourier series:

$$
\mathbf{M}_{\gamma}=\sum_{n=0}^{\infty}\left[\gamma_{n_{c}} \cos (n \psi)+\gamma_{n_{s}} \sin (n \psi)\right]
$$

When equation (3.5) is substituted in (3.6) and the coefficients of the various sine and cosine harmonics are equated the following equations result.

$$
\gamma_{0}=\text { mean yaw moment }=\frac{3 f_{1}}{2}
$$




$$
\begin{aligned}
& \gamma_{n_{c}}=\left\{\begin{array}{rr}
\frac{3}{2}\left[f_{(n-1)}-f_{(n+1)}\right] & n=3,6, \ldots \\
0 & n \neq 3,6, \ldots
\end{array}\right\} \\
& \gamma_{n_{s}}=\left\{\begin{array}{rr}
\frac{3}{2}\left[f_{(n-1)}-f_{(n+1)}\right] & n=3,6, \ldots \\
0 & n \neq 3,6, \ldots
\end{array}\right\}
\end{aligned}
$$

These results show the following important features for yaw moments resulting from blade flap loads.

1) The mean yaw moment results from the once-per-revolution (lp) sine flap moment only.

2) The only cyclic yaw moments due to balanced rotor loads on a three-blade rotor are moments with a frequency of $3 p$ or integer multiples of 3p. The 3p yaw moments result from $2 p$ and $4 p$ flap moments. This shift of plus or minus $1 p$ from the rotor frame of reference to the fixed (yaw) frame is well known.

Another important source of yaw moments is mass or aerodynamic imbalance of the rotor. If the rotor is not mass balanced then the centrifugal force due to the rotor mass will produce a yaw moment which depends upon the distance from the yaw axis to the hub $\left(L_{S}\right)$, the mass offset $(\overline{\mathrm{r}})$, the rotor mass $(\mathrm{m})$, and the rotor angular veiocity $(\Omega)$ :

$$
\mathrm{M}_{\gamma}=-\mathrm{L}_{\mathrm{s}} \mathrm{m} \tilde{\mathrm{r}} \Omega^{2} \sin \psi
$$

Note this is a $1 \mathrm{p}$ yaw moment. A similar $1 \mathrm{p}$ yaw moment will result if the blades are not aerodynamically balanced. Thus, if $1 \mathrm{p}$ yaw moments are observed during testing, there is probably a mass or aerodynamic (most likely blade pitch) imbalance. Observing the 1p yaw moments can be a very useful diagnostic tool for reducing cyclic yaw moments and/or balancing a rotor after installation. Imbalance is discussed in greater detail by Young, et al [Young, Hansen et al., 1988].

Figure 3.3 shows the result of a simple test to determine the importance of the root flap moments in determining the yaw moment for a rigid rotor. A ten-revolution (approximately 8 second) segment of data was selected from test records from the SERI Combined Experiment rotor. Both blade flap moment and yaw moment measurements were available. The blade flap data were azimuth averaged and then decomposed into a Fourier series to determine the coefficients $f_{n}$. Then equations $3.8,3.9$ and 3.10 were used to determine the coefficients $\gamma_{n}$. Next the $1 p$ yaw moment due to mass imbalance was determined from the known offset of the rotor center of gravity using equation (3.11). Finally, the yaw moment was computed from the resulting Fourier series for the flap moment and compared with the directly measured yaw moment. 


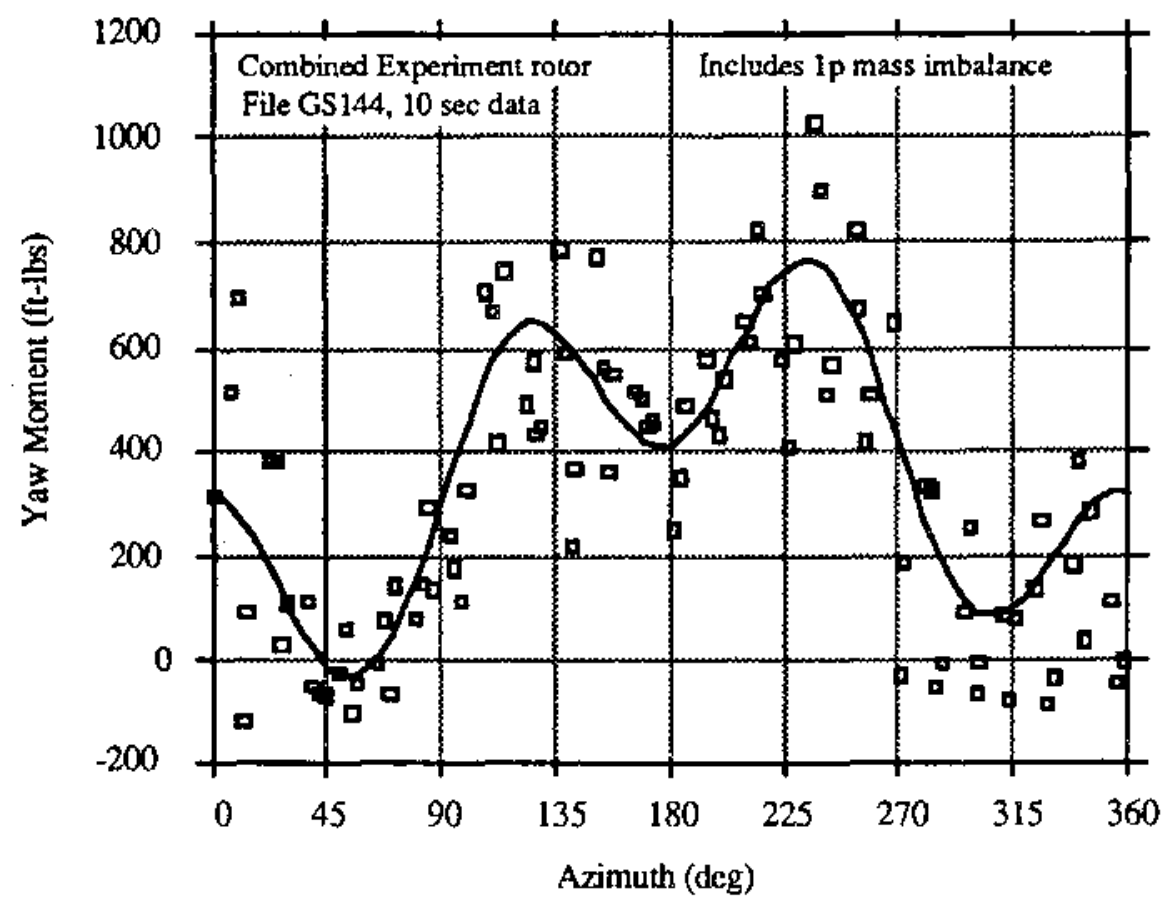

Figure 3.3. Comparison of the measured yaw moment (data points) with the moment calculated using the measured blade flap moment and the $1 \mathrm{p}$ rotor mass imbalance.

In Figure 3.3 the data points show the measured yaw moment and the solid line shows the yaw moment computed from the flap moments. It is clear that the reconstructed yaw moment agrees favorably with the actual moment. This implies that the horizontal $\mathrm{H}$-force is unimportant for this rigid rotor and that the nacelle aerodynamic moment is also negligible. It is also interesting to note the $1 \mathrm{p}$ harmonic is the largest contributor to the cyclic moment. Thus the mass imbalance is important to the overall yaw load spectrum of this particular rotor.

\subsection{Equations of motion of the teetering rotor}

The teetering rotor differs sufficiently from the rigid rotor that separate equations of motion are required. The derivation of the equations follows the same basic method presented for the rigid rotor. Details of the derivation are provided in Appendix A. Figure 3.4 shows the geometry and essential parameters of the teetering rotor. The equation governing the teetering motion is given by:

$$
\begin{array}{r}
\left(1-\frac{\mathrm{m}_{\mathrm{b}} \mathrm{s}^{2}}{\mathrm{I}_{\mathrm{b}}}\right) \mathrm{T}^{\prime \prime}=-\mathrm{T}-\frac{\mathrm{M}_{\text {aero }}+\mathrm{M}_{\text {hub }}}{2 \mathrm{I}_{\mathrm{b}} \Omega^{2}}-\frac{\mathrm{m}_{\mathrm{b}} g s \cos \psi}{\mathrm{I}_{\mathrm{b}} \Omega^{2}}-2 \gamma^{\prime} \cos \psi \\
-\gamma^{\prime \prime} \sin \psi\left(1+\frac{\mathrm{m}_{\mathrm{b}} \mathrm{L}_{\mathrm{s}} \mathrm{s}}{\mathrm{I}_{\mathrm{b}}}+\frac{\mathrm{m}_{\mathrm{b}} \mathrm{s}^{2}}{\mathrm{I}_{\mathrm{b}}}\right)
\end{array}
$$


The yaw motion of the teetering rotor is governed by the following equation:

$$
\begin{array}{r}
\gamma^{\prime \prime}\left[\begin{array}{l}
I_{\text {yaw }}\left(1-\frac{m_{b} s^{2}}{I_{b}}\right)+2 m_{b} L_{s}^{2}+4 m_{b} L_{s} s \\
-\cos ^{2} \psi\left\{2 m_{b} L_{s} s\left(2+\frac{m_{b} L_{s} s}{I_{b}}\right)+2 m_{b} s^{2}\left(1-\frac{m_{b} s^{2}}{I_{b}}\right)\right\}
\end{array}\right]= \\
-\frac{M_{\text {edgc }}}{\Omega^{2}}\left[\left(1-\frac{m_{b} s^{2}}{I_{b}}\right)(\tau+T \cos \psi)\right]-2 m_{b} L_{s} s T \sin \psi \\
-M_{\text {aero }} \frac{m_{b} L_{s} s}{I_{b} \Omega^{2}} \sin \psi+\frac{F_{z} L_{s} T}{\Omega^{2}} \sin \psi\left(1-\frac{m_{b} s^{2}}{I_{b}}\right) \\
-\frac{2 m_{b} g s \cos \psi \sin \psi}{\Omega^{2}}\left(1-\frac{m_{b} s^{2}}{I_{b}}+\frac{m_{b} L_{s} s}{I_{b}}\right) \\
+\frac{M_{\text {hub }} \sin \psi}{\Omega^{2}}\left(1-\frac{m_{b} s^{2}}{I_{b}}-\frac{m_{b} L_{s} s}{I_{b}}\right)-4 \gamma^{\prime} m_{b} L_{s} s \cos \psi \sin \psi \\
+4 T^{\prime} m_{b} s \cos \psi\left(L_{s}-s\right)\left(1-\frac{m_{b} s^{2}}{I_{b}}\right)
\end{array}
$$

In this equation $\mathrm{M}_{\text {hub }}$ is the net moment exerted on the hub by the teeter springs and dampers, $\mathrm{M}_{\mathrm{edge}}$ is the net aerodynamic torque on the rotor, $\mathrm{T}$ is the teeter angle and $\mathrm{s}$ is the undersling as shown in Figure 3.4.

The model contains provisions for simple teeter dampers and springs as shown in schematic form in Figure 3.4. The damper is a linear system which exerts a teeter moment proportional to the teeter rate (and opposing the teeter motion) for all teeter angles greater than the contact angle. The teeter spring is a nonlinear spring such that the teeter moment is a restoring moment given by equation 3.14 .

$$
M=k_{1} \varepsilon+k_{2} \varepsilon^{2}
$$

Here $\varepsilon$ is the deflection of the spring and $k_{1}$ and $k_{2}$ are constants which are input to the model. The deflection of the spring, $\varepsilon$, is the teeter angle minus a constant angle which is also input to the model (the free-teeter angle). Thus the rotor can teeter without mechanical restraint until the teeter angle exceeds a preselected value. 


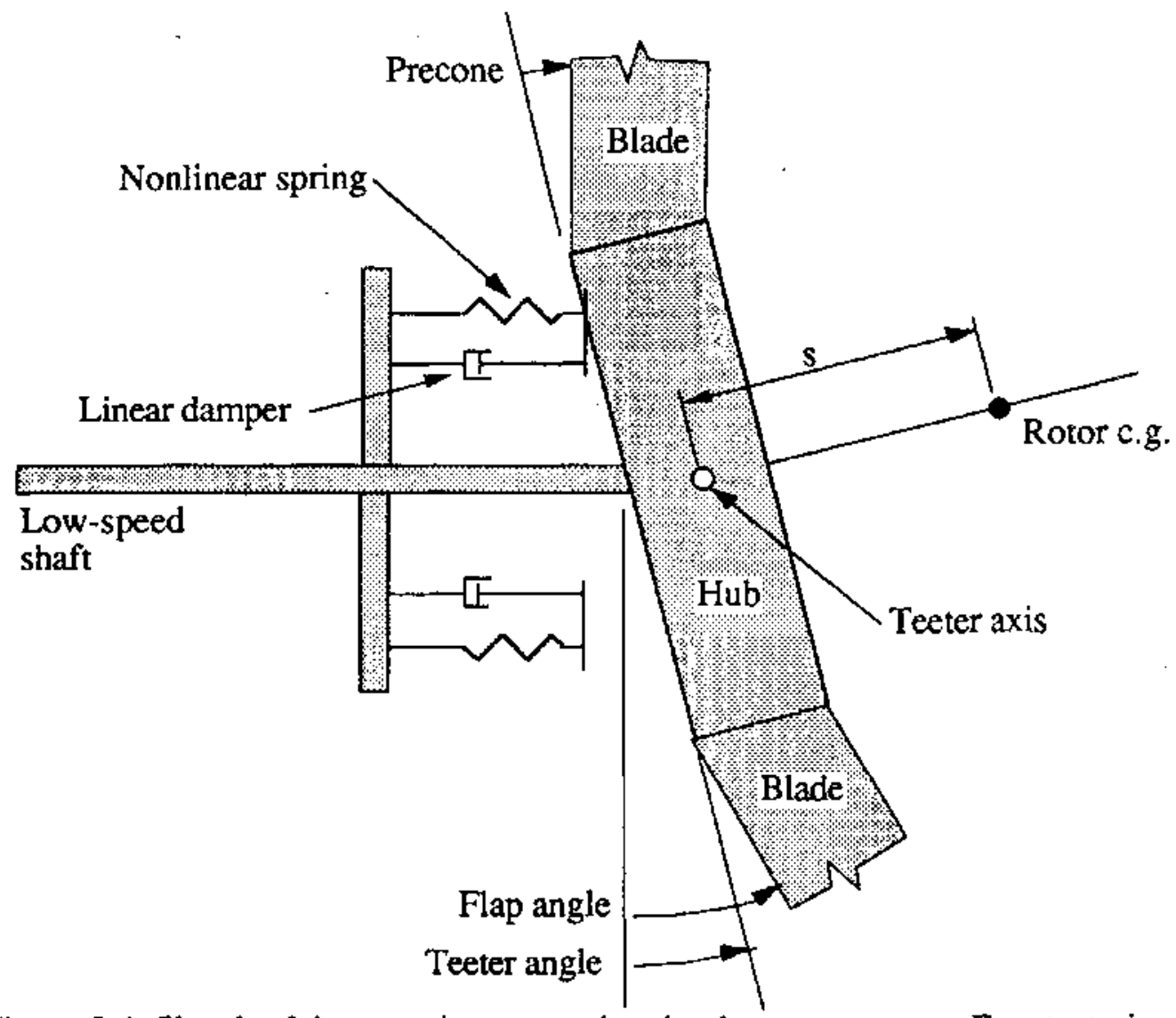

Figure 3.4 Sketch of the teetering rotor, showing key parameters. Free teetering is permitted until the spring and damper are contacted by the hub (in the position shown).

\subsection{Subsystem details}

\section{Yaw column}

The yaw column supports the nacelle and provides a bearing surface about which the nacelle is permitted to yaw. A constant applied moment (i.e., drag from yaw bearings) and a viscous drag moment proportional to the yaw rate are included in the model and provide the following damping moment

$$
\mathrm{M}_{\mathrm{d} \gamma}=-\mathrm{a}_{\mathrm{v}} \dot{\gamma}-\mathrm{a}_{\mathrm{f}} \operatorname{sign}(\dot{\gamma})
$$

where $\mathrm{a}_{\mathrm{v}}=$ viscous damping coefficient $\left(\mathrm{ft}_{\mathrm{f}}-\mathrm{lb}_{\mathrm{r}}-\mathrm{sec} / \mathrm{rad}\right)$

$\mathrm{a}_{\mathrm{f}}=$ moment to overcome bearing friction

The dry friction moment $a_{\mathrm{f}}$ exists only in the case where yaw motion exists. That is, there is no applied moment to the yaw column when the yaw rate is zero. This moment also always resists the motion, hence the signum function. No static friction is included in this model as yaw motions are assumed to be always present in the freeyaw machine even if they are exceedingly small.

A yaw stiffness is also provided in the model for those situations where the nacelle is nominally held fixed in yaw. The stiffness represents the effective torsional stiffness of the connection from the mainframe to ground. Thus it includes the yaw drive, yaw 
column and tower stiffness. The yaw moment exerted by this "spring" is simply the yaw deflection (from the initial yaw angle) multiplied by the yaw stiffness.

\section{Rotor Aerodynamics}

The blade element/momentum method has been the most useful form of aerodynamics analysis for wind turbine designers. The method offers accuracy, simplicity and ease of intuitive understanding. This is the method selected for the yaw analysis, for the same reasons. The basic method and equations used in this analysis are virtually identical to those detailed by Wilson and Lissaman [Wilson and Lissaman, 1974]. The method includes the static stall model for very high angles of attack developed by Viterna [Viterna and Corrigan, 1981].

However, previous investigators (notably in wind turbine work, [de Vries, 1985]) have shown that simple blade element/momentum methods will predict yaw moments which are less than actual moments and insufficient to cause the yaw stability that is observed on many turbines. Helicopter analysts have noted the same shortcoming in studies of roll and pitch stability in forward flight [Prouty, 1986]. Fortunately, a significant amount of work has been done on this problem by helicopter aerodynamicists. We can apply this work directly to the wind turbine rotor in yaw.

Coleman, et al [Coleman, Feingold et al., 1945] first noted that a skewed wake will perturb the induced velocity field from that which would be expected from blade element theory. They calculated the magnitude of the perturbation and found that induced velocities would be reduced at the upstream edge of the rotor and increased at the downwind edge of the rotor. They also noted a nearly linear variation of induced velocity along the axis aligned with the flight direction (or along a horizontal line through the rotor hub for a yawed wind turbine). Blade element theory is not completely accurate because it assumes independence of all the elements. That is, it assumes that the induced velocity at a particular blade element depends only upon the force on that single element. In fact, the induced velocity field depends upon the distribution of vorticity in the entire wake. This effect becomes important when the wake is no longer symmetric. In a skewed wake the blade elements on the downwind side of the rotor are closer to the wake centerline than are the elements on the upwind side of the rotor. Hence the induced velocities are higher on the downwind side than on the upwind side.

Pitt and Peters [Pitt and Peters, 1981] introduced a method for calculating this effect which is self-consistent in both forward and vertical flight. Gaonkar and Peters [Gaonkar and Peters, 1986] have provided a recent survey of this topic and comparisons with test data that show the method of Pitt and Peters is valid. This is the method that was first applied by Swift [Swift, 1981] to the wind turbine. Swift used an actuator-disc analysis with the skewed wake correction being a linear variation superimposed upon a constant induced velocity. 
The method used in the current work is aciapted to a blade element analysis and uses the following equation to adjust the axial induction factor, " $a$ ".

$$
\mathrm{a}=\mathrm{a}_{0}\left[1+\frac{15 \pi}{32} \sqrt{\frac{1-\cos \gamma}{1+\cos \gamma}} \frac{\mathrm{r}}{\mathrm{R}} \mathrm{K} \sin \psi\right]
$$

Where $\quad \mathbf{a}=$ axial induction factor used to determine actual induced velocity

$\mathrm{a}_{0}=$ axial induction factor calculated using simple blade

element/momentum theory

Note the dependence upon yaw angle, $\gamma$, and radial position. When there is zero yaw, no correction is applied. The variation along a horizontal line $\left(\psi=90^{\circ}\right)$ is linear with radius. For small yaw angles the yaw dependence is approximately linearly proportional to $\gamma$. The factor $\mathrm{K}$ is included only for sensitivity studies in the computer programs. The theory of Pitt and Peters predicts $K=1$. This is the value used in the final version of YawDyn.

\section{Dynamic Stall}

The Combined Experiment rotor has the capability of measuring the angle of attack using a small vane and measuring the lift coefficient using a chordwise distribution of pressure transducers. Figure 3.5 presents such data taken at the $80 \%$ span station. The data represents ten revolutions of the rotor, with approximately eight samples measured per revolution (10 Hz sampling frequency). Sequential samples are connected using solid lines to show the hysteresis loop present in the $\mathrm{CL}$ curve. The hysteresis loop progresses clockwise around the figure as time increases. Two-dimensional wind tunnel measurements of steady $\mathrm{CL}_{\mathrm{L}}$ values are shown for comparison. Notice the $\mathrm{CL}_{\mathrm{L}}$ decreases below static test values as the angle of attack rapidly decreases from its maximum value during yawed operation.

Attempts to predict yaw loads on the Combined Experiment using YawDyn were unsuccessful when this stall hysteresis was not included in the model. This prompted efforts to incorporate a dynamic stall model in YawDyn. The model selected is the stall hysteresis analysis proposed by Gormont [Gormont, 1973].

Gormont developed a method for treating dynamic stall in helicopter analysis. Sandia National Laboratories has adopted this method for analysis of vertical axis wind turbine dynamic stall [Berg, 1983]. (The complete Gormont model includes dynamic inflow. This portion of the model was not implemented in the present work.) The Gormont model calculates a lift coefficient based upon static two-dimensional wind tunnel values and the time rate of change of angle of attack. A modified blade angle of attack, $\alpha_{m}$, is 


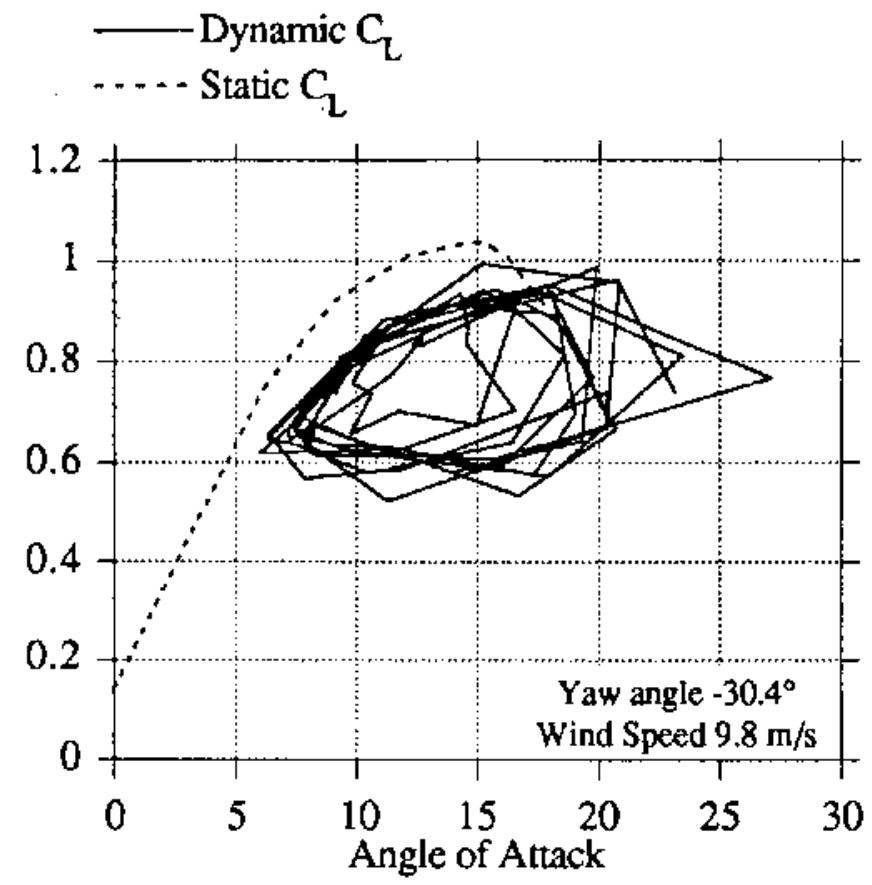

Figure 3.5. Stall hysteresis loop measured at the $80 \%$ span on the Combined Experiment rotor.

used which depends upon the actual angle of attack, $\alpha_{b}$, and $d \alpha_{b} / d t$ as follows:

$$
\alpha_{\mathrm{m}}=\alpha_{\mathrm{b}}-\mathrm{K}_{\mathrm{J}}\left[1.4-6.0\left(0.06-\frac{\mathrm{l}}{\mathrm{c}}\right)\right]\left[\frac{\mathrm{c} \dot{\alpha}}{2 \mathrm{U}_{\mathrm{r}}}\right]^{\frac{1}{2}} \operatorname{Sign}(\dot{\alpha})
$$

In this equation $c$ is the blade chord, $t$ is the blade thickness, and $U_{r}$ is the local relative wind speed. $\mathrm{K}_{1}$ is a constant which assumes either of two values depending upon the sign of $d \alpha_{b} / d t$ in the following manner.

$$
\mathrm{K}_{1}=\left\{\begin{array}{ll}
\mathrm{A} & \alpha_{\mathrm{b}} \frac{\mathrm{d} \alpha_{\mathrm{b}}}{\mathrm{dt}}>0 \\
\mathrm{~B} & \alpha_{\mathrm{b}} \frac{\mathrm{d} \alpha_{\mathrm{b}}}{\mathrm{dt}}<0
\end{array}\right\}
$$

The standard Gormont model assigns values of $A=1$ and $B=0.5$. Parameter $A$ determines the amount of increase in maximum $\mathrm{CL}_{\mathrm{L}}$. $\mathrm{B}$ affects the size of the hysteresis loop. The Combined Experiment data of Figure 3.5 demonstrate no increase in maximum $C_{L}$. Therefore $A=0$ was used in most of the calculations which will be presented. 
Once $\alpha_{m}$ is determined the effective $\mathrm{C}_{\mathrm{L}}$ is calculated using the following equation:

$$
C_{L}=\frac{\alpha_{b}-\alpha_{b 0}}{\alpha_{m}-\alpha_{b 0}} C_{L}\left(\alpha_{m}\right)
$$

Where $\alpha_{b 0}$ is the zero-lift angle of attack and $\mathrm{Cl}_{\mathrm{L}}\left(\alpha_{\mathrm{m}}\right)$ is the two-dimensional wind tunnel static value of $\mathrm{CL}$ at angle $\alpha_{\mathrm{m}}$.

To summarize, the induced velocity field is calculated in a three-step process. First the axial induction factor, a0, is calculated using the blade element/momentum method. Second, the induction factor is adjusted using equation 3.16 to account for the skewed shape of the wake. Third, dynamic stall theory is used to adjust the section lift coefficient and then the blade forces are calculated using the blade element method and the new value of axial induction factor.

\section{Wind Shears}

The wind is represented as a velocity varying in magnitude and direction. Temporal variations in the wind vector can be analyzed by reading a wind data file. Spatial variations in the wind speeds are represented by wind shear coefficients which can also vary in time. The wind direction is assumed constant over the rotor disc (i.e. there are no wind direction shears considered in the model). The spatial variations are caused by complex terrain or arrays of turbines upwind of the HAWT (persistent, long-term shear) and also by atmospheric turbulence ("instantaneous" shear). This model treats the shears as linear variations in the wind speed in both the horizontal and vertical directions as shown in Figure 3.6. An option is also available to use power-law wind shear in the vertical direction. A vertical component of wind velocity is also included in the model. This value is a constant across the entire rotor disc (note that a positive vertical wind blows toward the ground with the present coordinate system). The hub height wind input consists of an instantaneous horizontal speed $V_{\infty}$ and direction $\delta$ with respect to the $\mathbf{z}$ axis. The angle $\delta$ can vary independent of the yaw angle $\gamma$. The yaw error, or misalignment of the rotor from the wind direction is $\gamma+\delta$. Both $\gamma$ and $\delta$ are shown in the positive direction in Figure 3.6.

\section{Tower Shadow}

The downwind HAWT has a region, represented by a sector centered at the blade six o'clock position, through which each rotor blade will encounter the wake of the tower. Within this region, the so-called tower shadow, the wind velocity is altered by turbulence and vorticity. The total effect this has on the aerodynamic loading on the blade is quite complicated. To determine the importance of the tower shadow, it is modeled by assuming the velocity normal to the rotor disc within this region is reduced by a factor that is a function of the blade azimuth angle. Experimental measurements [Hoffman, 1977; Savino and Wagner, 1976] within the near wake region of the tower indicate this velocity deficit has a magnitude which can be $30 \%$ to $50 \%$ of the undisturbed flow. Values of 5-10\% are more typical if the blade is in the far wake. 

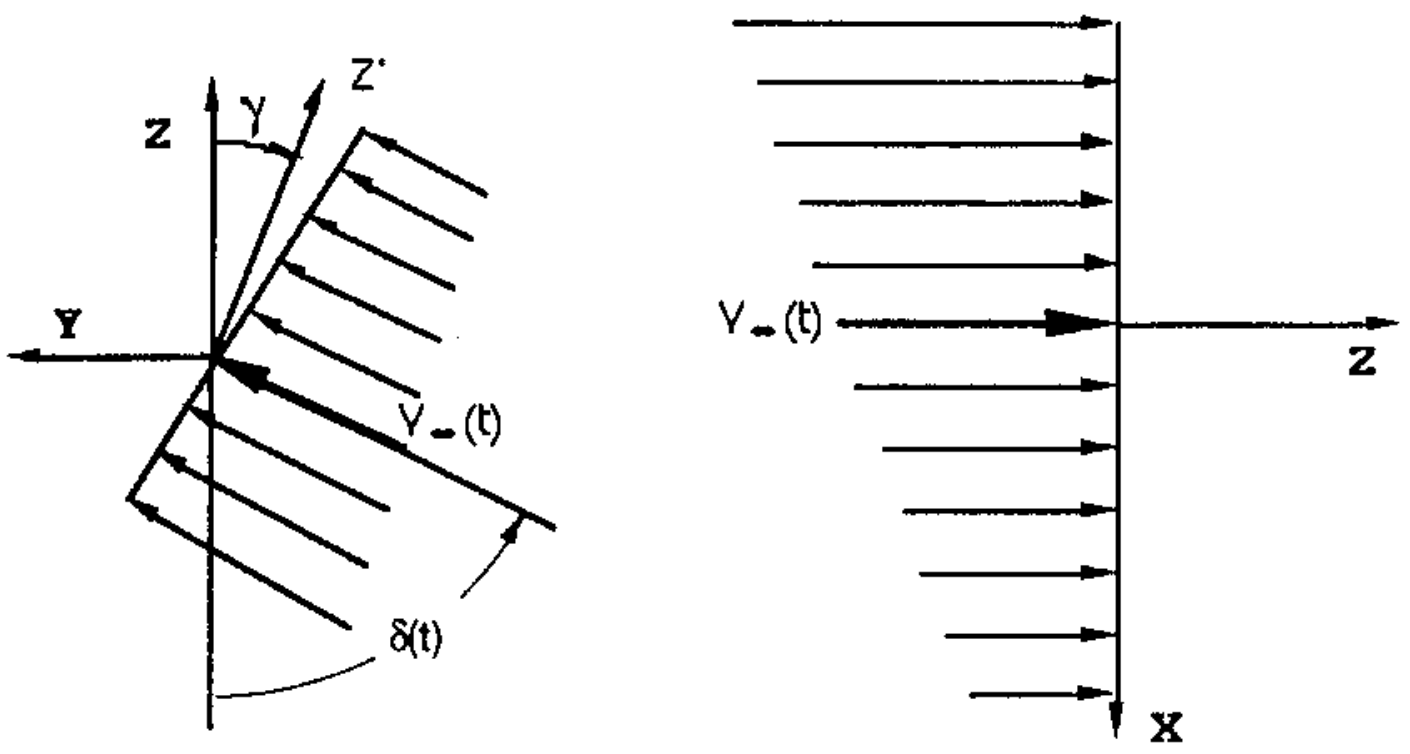

Figure 3.6 Linear wind shear models. Horizontal shear in left sketch, vertical shear in right sketch. (Vertical shear can also be a power-law profile.)

The following expression gives the normal wind speed in terms of the speed which would be present if there were no tower shadow:

$$
\mathrm{V}_{\mathrm{n}}(\mathrm{x}, \mathrm{y}, \mathrm{z}, \psi)=\mathrm{V}_{\mathrm{n}}(\mathrm{x}, \mathrm{y}, \mathrm{z})\left[1-\mathrm{T}_{\mathrm{s}}(\psi)\right]
$$

The tower shadow shape function is:

$$
\begin{aligned}
& \mathrm{T}_{\mathrm{s}}(\psi)=\left\{\begin{array}{cc}
\frac{\Delta \mathrm{V}_{s}}{2}\left[1+\cos \left(\pi \frac{\psi}{\psi_{0}}\right)\right] & \text { if }-\psi_{0} \leq \psi \leq \psi_{0} \\
0 & \text { elsewhere }
\end{array}\right\} \\
& \Delta \mathrm{V}_{\mathrm{s}}=\text { velocity deficit ratio at the tower shadow centerline } \\
& \Psi_{0}=\text { half-angle of tower shadow sector }
\end{aligned}
$$

In the final version of YawDyn the sector containing the tower shadow has an included angle of $2 \psi_{0}=30^{\circ}$. Notice this "wedge shape" wake is not representative of all tower shadows, and the model always centers the wake at $\psi=0^{\circ}$, regardless of the yaw angle. In fact, at large yaw angles, the blade may not enter the wake at all. Nonetheless, the simple model is useful for approximating the importance of the shadow. Future models will implement a more refined model of the tower shadow. 


\subsection{Numerical Solution}

The coupled, nonlinear ordinary differential equations governing yaw and flap motion are far too complex to be solved analytically. Instead, numerical integration is performed for the equations. This approach is less than ideal because it makes completion of sensitivity studies and identification of trends tedious. But it offers the overwhelming advantage that it is possible to consider all of the important nonlinear effects (such as static and dynamic stall) and the details of the turbine (such as blade twist and taper, nonlinear teeter-stop springs, etc.).

\subsection{Numerical integration}

The primary method of integration is the Modified Adams-Bashforth PredictorCorrector. This method was selected for speed, stability and accuracy and is commonly used in problems of this type. The ordinary differential equations are first arranged as a series of " $n$ " first order differential equations.

The Adams-Bashforth algorithm requires four sets of starting points $\left\{x_{\mathrm{x}, \mathrm{j}}\right\},\left\{\mathrm{x}_{\mathrm{k}, \mathrm{i}-\mathrm{i}}\right\}_{\mathrm{r}}$ $\left\{x_{k, i-2}\right\}$, and $\left\{x_{k, j-3}\right\}$ at times $t_{i}, t_{i-1}, t_{i-2}$, and $t_{i-3}$ where $k=1,2, \ldots n, t_{j}=t_{j-1}+h$ and $h$ is the fixed step size. In addition, the function values $\left\{F_{k, i}\right\},\left\{F_{k, i-1}\right\},\left\{F_{k, i-2}\right\}$, and $\left\{F_{k, i-3}\right\}$ are required, where $F_{j, i}$ is the jth element of the function vector, evaluated at time $\mathrm{t}_{\mathrm{i}}$ and using vector $\left\{\mathrm{x}_{\mathrm{k}, \mathrm{i}}\right\}$. Once these starting points are obtained, by a simple predictor-corrector method, perform the following:

Step 1. Find $\mathrm{pk}_{\mathrm{k}}=\mathrm{x}_{\mathrm{k}, \mathrm{i}}+\mathrm{h} / 24\left(55 \mathrm{~F}_{\mathrm{k}, \mathrm{i}}-59 \mathrm{~F}_{\mathrm{k}, \mathrm{i}-1}+37 \mathrm{~F}_{\mathrm{k}, \mathrm{i}-2}-9 \mathrm{~F}_{\mathrm{k}, \mathrm{i}-3}\right)$

Step 2. Find $c_{k}=x_{k, i}+h / 24\left(9 F p_{k, i+1}+19 F_{k, i}-5 F_{k, i-1}+F_{k, i-2}\right)$

Step 3. Find $x_{k}, i+1=1 / 270\left(251 c_{k}+19 p_{k}\right)$

In these equations $\mathrm{F}$ is the function on the right-hand side of the first-order differential equations and $F^{P}, i+1$ is the $k$ th element of $F$, evaluated by replacing $x_{i+1}$ in the argument by $\mathrm{pk}_{\mathrm{k}}$. This solution method requires evaluation of the right-hand side only once per time step. This is the reason the solution is faster than many other methods, such as the Runge-Kutta techniques.

The dynamic stall model requires evaluation of the time-rate-of-change of the blade element angle of attack. Numerical differentiation is inherently "noisy" and the iteration to determine the induced velocities is highly dependent upon the airfoil lift coefficient. Early attempts to implement the Gormont dynamic stall model were unsuccessful because of numerical instabilities in the angle of attack estimation. This problem was solved by smoothing the angle of attack time history before calculating the time derivative. Smoothing was accomplished using a Sine-Butterworth digital low pass filter. The filtered angle of attack is used only for estimating the time derivative.

\section{4,2_Initial conditions_and trim solution}

The computer program starts with the initial conditions specified by the user and integrates through two complete revolutions of the rotor while wind and yaw conditions remain constant. It then compares the blade motions for the two revolutions. If the root-mean-square difference between the flap angles for the two revolutions are below a 
specified tolerance for each blade the "trim" solution has been found. If the trim solution has not been found another revolution is calculated. This process continues until the two most recent revolutions yield the same flap motion (within the tolerance). After the trim solution is found the program allows the simulated time to reset to zero and the integration proceeds with the actual time-varying yaw angle and wind conditions.

The integration requires an initial condition for each of the degrees of freedom of the system. In the general case the flap angle and flap rate for each blade and the yaw angle and yaw rate of the system must be specified by the user of the program. The selection of these initial conditions will have a large influence on the time required to find the trim solution for the rotor. If the flap initial conditions are too different from the conditions that would actually exist on the rotor the program may not be able to converge to a trim solution. This is particularly true for blades which have a low stiffness and are therefore experiencing large deflections. Thus it is important that the user understand the rotor dynamics and have some idea of the blade flap motion that would be expected under the selected operating conditions.

If the blade stiffness is quite high, say greater than $3 p$, it is often sufficient to use initial conditions for each blade which set the flap angles equal to the precone angle and the flap rates to zero. For "soft" blades it may be necessary to specify different angles and rates for each blade. This can almost always be achieved by a few trial and error runs. The program informs the user of the trim solution initial conditions to help guide the selection of appropriate values. In the most difficult cases it may be necessary to vary operating conditions slowly from a known solution to the desired, difficult condition. For example, if the yaw angle is very large it may be difficult to guess a set of initial conditions which will converge to a trim solution. In that case it may be necessary to run the program in the fixed-yaw mode for a series of progressively increasing yaw angles (starting from low values where the trim is easy) and observe the trend in the trim solution initial conditions. This trend can be used to extrapolate the initial conditions for a new yaw angle.

\subsection{Program structure and flow chart}

Figure 4.1 shows a summary flow chart of YawDyn. More details are found in the User's Guide in Appendix C.

\subsection{Computer requirements}

The program was developed with the intent that it would be suitable for tradeoffs and sensitivity studies. This meant it had to run quickly on relatively simple and low-cost computers. Though the computing requirements have grown as more physical phenomena were added to the program, the personal computer capabilities have grown at an even faster rate. Thus the program is still quite suitable for design and tradeoff calculations using hardware available to any small business.

Most of the development of YawDyn has been done using Macintosh II $^{\mathrm{TM}}$ computers using Absoft ${ }^{\mathrm{TM}}$ MacFortran II. The program will run on these systems with $2 \mathrm{MB}$ of RAM. Other versions of Fortran 77 will run on the Macintosh with 1 MB RAM. Typical run times on a Macintosh IIci system are one to five minutes. The program has also been tested under VAX VMS and IBM PCs (clones) using Lahey F77L ${ }^{\mathrm{TM}}$ Fortran and NDP Fortran. System requirements are minimal though the run times are long enough that there is incentive for using a relatively fast personal computer. 


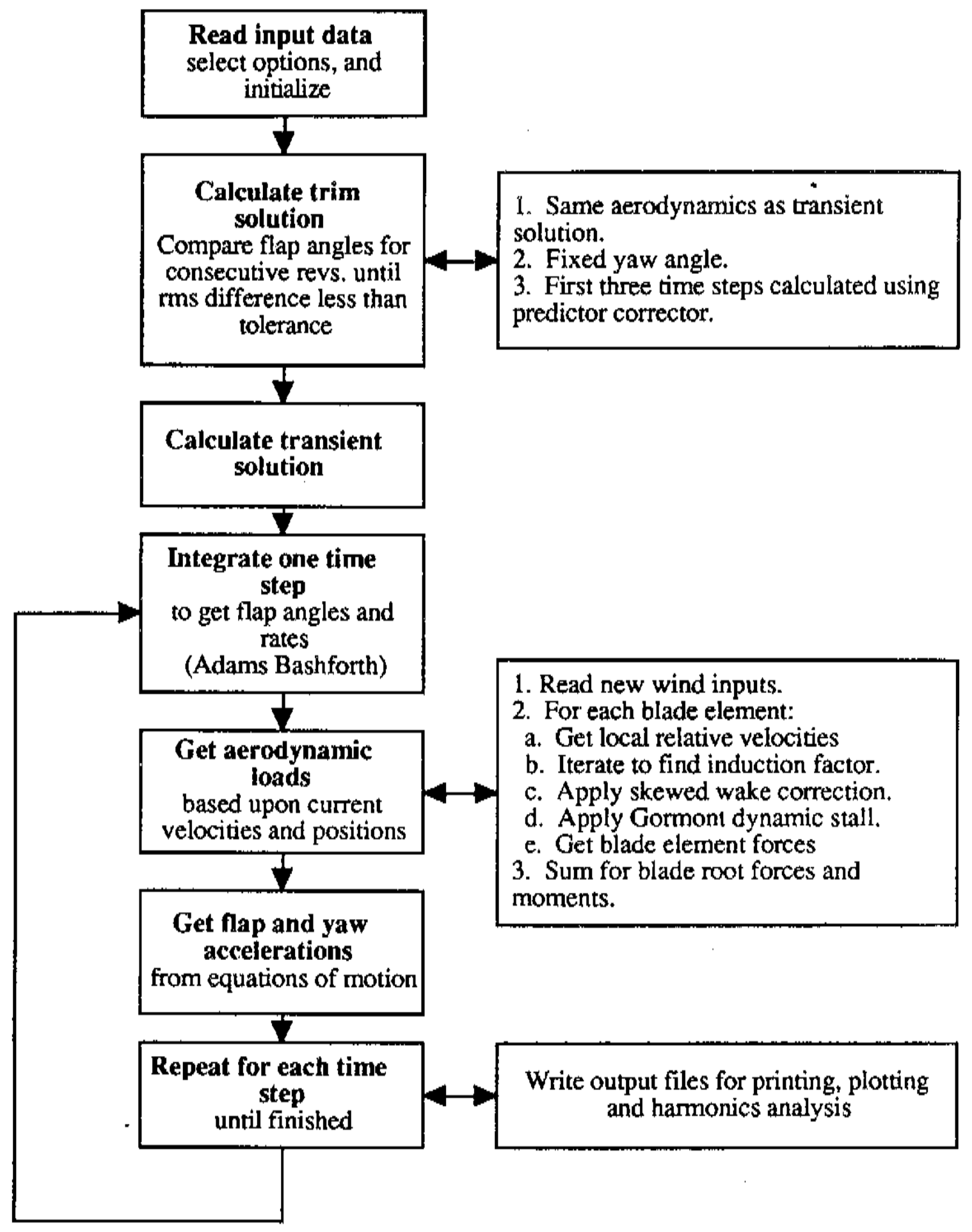

Figure 4.1 Summary flow chart of the YawDyn computer program. 


\subsection{Validation Studies}

\subsection{Introduction}

One of the most important tasks of the project was validation of the YawDyn model. Several methods were explored to find means of testing the validity of the predictions. The wind energy research field is hampered by a scarcity of complete and reliable test data (particularly for yaw loads and motions). However, two sets of very helpful data were identified and used for testing the computer predictions. Where data could not be found for testing the model, comparisons with predictions of other models were made. This offers the advantage of permitting detailed exploration of the analysis assumptions and options but has the obvious disadvantage that comparisons are made with results which are not proven accurate.

The following sections present comparisons with wind-tunnel measurements, field test data, and predictions of the SERI FLAP code. An attempt has been made to test the limits of the code and find its strengths and weaknesses. Certainly both strengths and weaknesses have been found. Testing and refinement of the computer program will continue, so this section reports a "snapshot" of the status of the method. Hopefully, this will stimulate other researchers to improve the model and provide designers with some indications of the reliability of the predictions and insights which can be obtained from the model.

\subsection{Mod-2 Wind Tunnel Test Comparisons}

Early in the development of the Mod-2 wind turbine a series of wind-tunnel tests were conducted on a 1/20 scale model [Shipley, 1978]. Two configurations were tested, one with a rigid hub and the other with a teetering hub. The rigid-hub tests provide a good data set for comparison with YawDyn. The rotor was tested at three yaw angles and three wind speeds, each with a vertical shear (but no other non-uniformity) in the approach wind. The pitch angle was adjusted at each wind speed to limit power output. The angle of attack at the tip of the blade typically averaged near $0^{\circ}$ in all test cases. Thus the data do not provide insight to deep stall operation of the rotor. Nevertheless, the availability of detailed and controlled test data was very helpful.

Early comparisons with the test data were not encouraging[Hansen and Cui, 1989; Cui, Hansen, et al, 1988]. But investigation of the poor agreement between predictions and measurements lead to the incorporation of skewed wake effects in the model. Then the predictions matched the data quite well, both qualitatively and quantitatively.

Figures 5.1 and 5.2 show comparisons of both mean and cyclic yaw moments. It can be seen that the qualitative trends are in excellent agreement. Note the yaw moment is negative at all wind speeds when the yaw angle is $0^{\circ}$. This means the rotor will not be statically stable directly upwind. The sign of the yaw moment changes with wind speed for negative yaw angles. Thus the stability will depend on the wind speed and the yaw angle. Clearly this rotor would not be satisfactory as a free-yaw, upwind system. It is also interesting to note the cyclic yaw moments are several times larger than the mean moments. This means the yaw loads are fully reversing fatigue cycles on the yaw drive and that peak loads will be much larger than mean loads.

The quantitative agreement is less satisfactory, but still quite encouraging. The mean yaw moments appear to differ by a nearly constant offset. The predicted yaw moment 
exceeds the measured value by 3 to $5 \mathrm{ft}-1 \mathrm{lbs}$ (about 20-30\% of the largest moment measured). The $2 \mathrm{p}$ moments show better agreement at $+20^{\circ}$ yaw angle than at $-20^{\circ}$. The reasons for these discrepancies are not known. This level of accuracy is in large part due to the incorporation of the skewed wake corrections to the basic bladeelement/momentum aerodynamics.

Dynarnic stall effects were included in the calculations but made little difference in the results. This is because most of the blade never stalled during these tests.

Table 5.1 lists the pertinent conditions for the predictions above. Other machine characteristics are given in Appendix B.

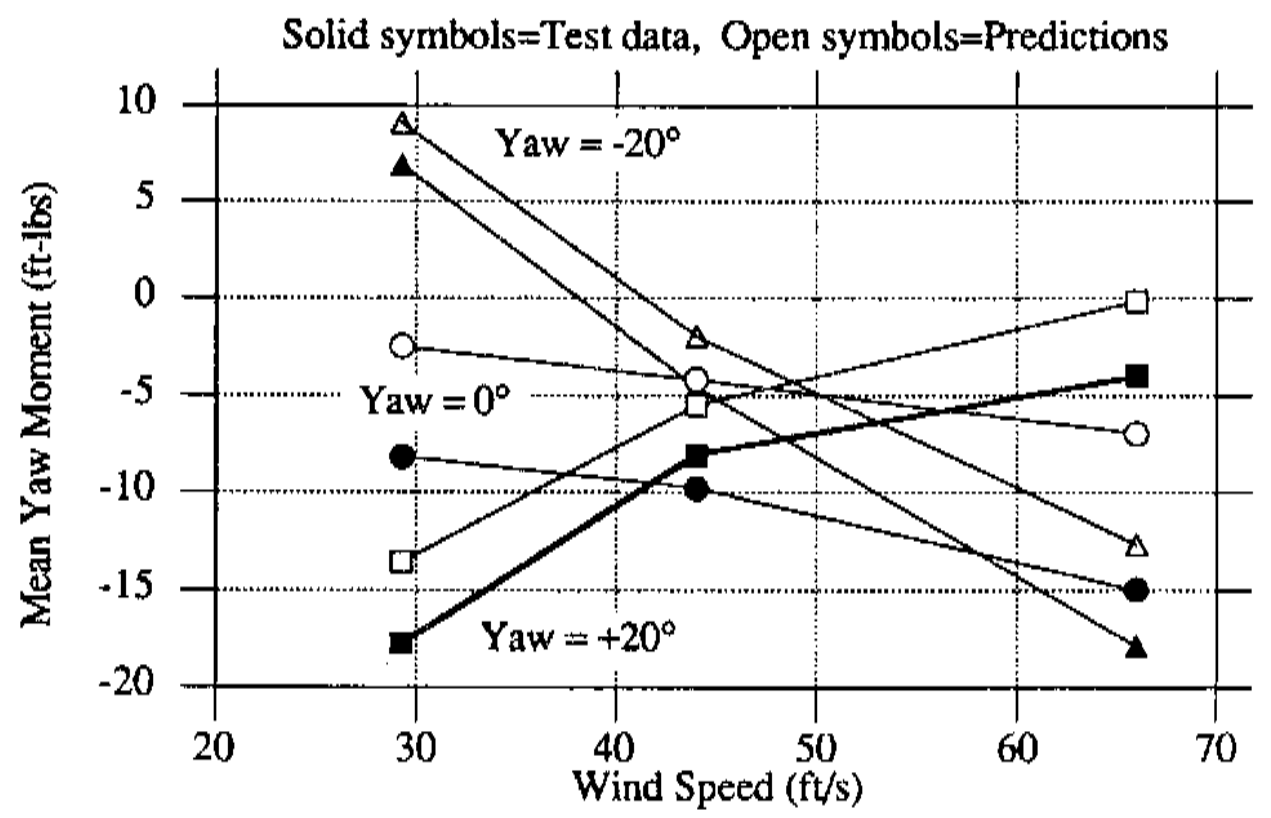

Figure 5.1. Comparison of predicted and measured mean yaw moments for the windtunnel model of the rigid-rotor Mod-2. 


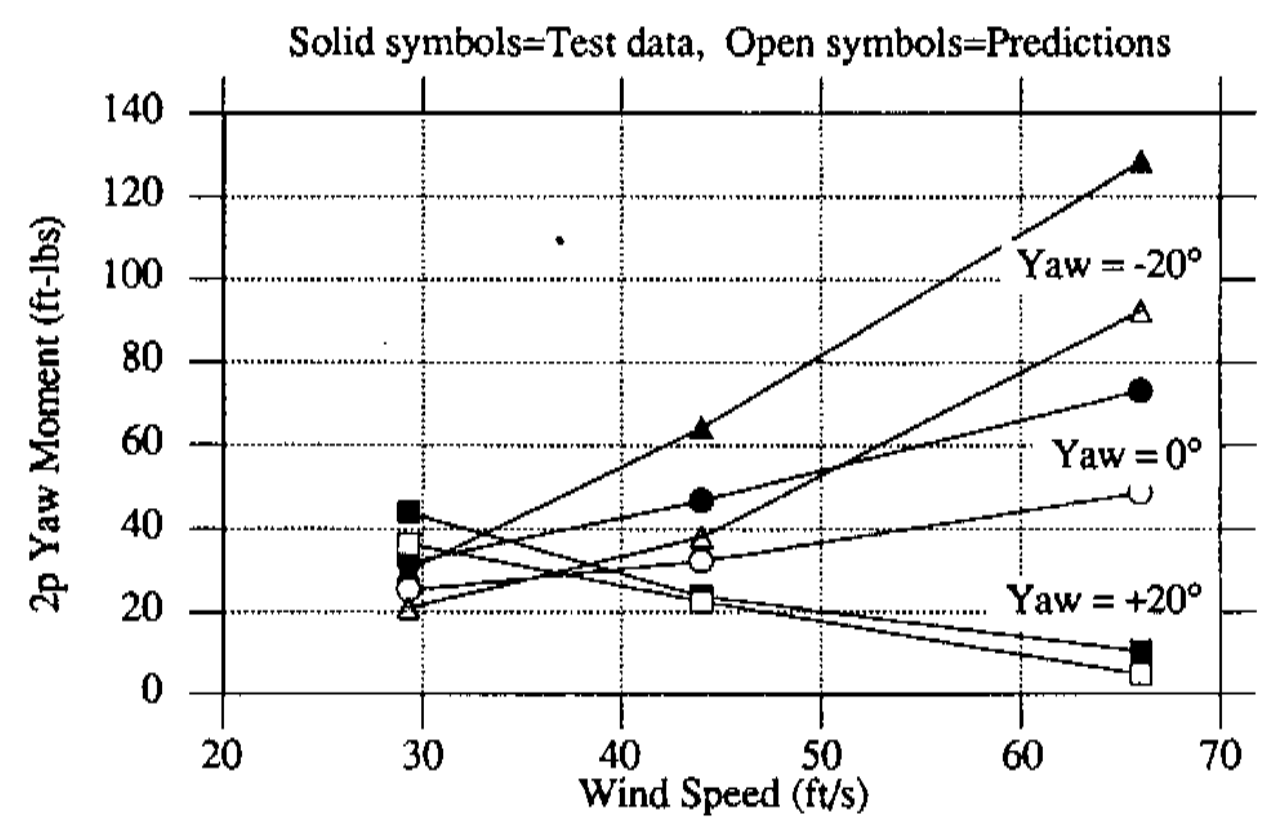

Figure 5.2. Comparison of predicted and measured 2 p yaw moments for the windtunnel model of the rigid-rotor Mod-2.

Table 5.1 Parameters used in the analysis of the Mod-2 wind-tunnel model.

\begin{tabular}{|l|l|}
\hline \multicolumn{1}{|c|}{ Parameter } & \multicolumn{1}{c|}{ Value } \\
\hline \hline Yaw angle & Fixed \\
\hline Flap DOF? & Yes \\
\hline Hub & Rigid \\
\hline Pitch angles (all blades) & $0^{\circ} @ 29.3 \mathrm{ft} / \mathrm{s}$ \\
& $9.3^{\circ} @ 44 \mathrm{ft} / \mathrm{s}$ \\
& $18^{\circ} @ 66 \mathrm{ft} / \mathrm{s}$ \\
\hline Dynamic stall & $\mathrm{A}=0.5, \mathrm{~B}=0.5$ \\
& Filter 2 stages at $20 \mathrm{p}$ \\
\hline Number of sectors & 200 \\
\hline Convergence tolerance & 0.01 \\
\hline Vertical shear & 0.14 power law \\
\hline Horizontal shear & 0.0 \\
\hline Vertical wind component & 0.0 \\
\hline
\end{tabular}




\subsection{SERI Combined Experiment and FLAP Prediction Comparisons}

The Combined Experiment rotor operated by SERI[Butterfield, 1989a; Butterfield, $1989 \mathrm{~b}$ ] is perhaps the most thoroughly and carefully instrumented rotor available for understanding aerodynamic and dynamic loads on a wind turbine. It has been the main vehicle for the validation of YawDyn and the SERI FLAP code. In addition to blade, yaw column and low-speed shaft load strain gages, there are numerous pressure taps for airload measurement, angle-of-attack sensors and a vertical-plane array of anemometry upwind of the rotor. This instrumentation has made it possible to explore and document the details of the dynamic loads and dynamic stall occurring on the blades. These tests have clearly demonstrated the existence and importance of dynamic stall in the operation of a wind turbine and were the motivation for including dynamic stall effects in the YawDyn model.

Table 5.2 lists many of the parameters of the YawDyn model which were used in the predictions that follow. Other details of the Combined Experiment rotor are provided in Appendix B. The dynamic stall constants were selected to give good agreement with the data. Note that no dynamic overshoot was included in the dynamic stall but a lower hysteresis loop larger than that suggested by Gormont $(B=0.5)$ was required. One of the weaknesses of the Gormont model is its dependence upon test data for selection of the hysteresis parameters.

Table 5.2 Parameters used in the analysis of the Combined Experiment Rotor.

\begin{tabular}{|l|l|}
\hline \multicolumn{1}{|c|}{ Parameter } & \multicolumn{1}{c|}{ Value } \\
\hline Yaw angle & Fixed \\
\hline Flap DOF? & Yes \\
\hline Hub & Rigid \\
\hline Pitch angles (all blades) & $11.3^{\circ}$ for data set $901-1$ \\
& $11.2^{\circ}$ for data set $901-2$ \\
& $11.5^{\circ}$ for data set $901-3$ \\
\hline Dynamic stall & A=0.0, B=0.7 \\
& Filter 2 stages at $20 \mathrm{p}$ \\
\hline Number of sectors & 200 \\
\hline Convergence tolerance & 0.01 \\
\hline Vertical shear & Input from data file \\
\hline Horizontal shear & Input from data file \\
\hline Verical wind component & Input from data file \\
\hline
\end{tabular}

In all of the predictions, measured wind data were input to the calculations from the 'YawDyn.wnd' file. Instantaneous wind speed, wind direction, vertical wind component, and horizontal and vertical wind shear coefficients were used in the calculations. No attempt was made to lag the wind data to allow for the convection time from the vertical plane array to the rotor. Table 5.3 summarizes the conditions for the 
three data sets which were used in the validation. Note the test durations are quite short, leading to small standard deviations on all wind characteristics.

Table 5.3 Wind characteristics from the Combined Experiment data sets. Values are Mean \pm Standard Deviation.

\begin{tabular}{|l|c|c|c|}
\hline \multicolumn{1}{|c|}{ Characteristic } & Data set 901-1 & Data set 901-2 & Data set 901-3 \\
\hline \hline Test duration (sec) & 9.81 & 5.71 & 4.03 \\
\hline Wind speed (ft/s) & $36.3 \pm 1.2$ & $34.9 \pm 0.9$ & $52.0 \pm 0.9$ \\
\hline Wind Direction (deg) & $305 \pm 3.8$ & $282.4 \pm 4.1$ & $283 \pm 1.8$ \\
\hline Yaw Angle (deg) & $315.9 \pm 0.1$ & $315.6 \pm 0.1$ & $259 \pm 0.1$ \\
\hline Vertical shear & $-0.043 \pm 0.18$ & $-0.032 \pm 0.17$ & $-0.053 \pm 0.068$ \\
\hline Horiz. shear & $0.045 \pm 0.076$ & $-0.027 \pm 0.08$ & $0.054 \pm 0.076$ \\
\hline Vertical wind (ft/s) & $-0.092 \pm 0.67$ & $-0.54 \pm 1.3$ & $-0.18 \pm 0.46$ \\
\hline
\end{tabular}

Flap Moment Predictions. First examine the blade root flap moments. Since the yaw loads depend almost exclusively on the flap moments for a rigid rotor, it is important to determine the accuracy of the flap load calculations. It is best to begin with a comparison of the YawDyn and FLAP models. This permits isolation of specific phenomena and establishes a baseline for comparison with test data. Figure 5.3 compares the predictions for a simple case where tower shadow and vertical shear are the only excitation of the flap motion. When the models use equivalent tower shadow representations they produce virtually identical results. This demonstrates that YawDyn is capable of predicting flap moments as well as FLAP, with its more sophisticated model of the structural dynamics. It also demonstrates the importance of the tower shadow in determining the amplitude of the $4 p$ response [Hansen and Wright, 1991]. 


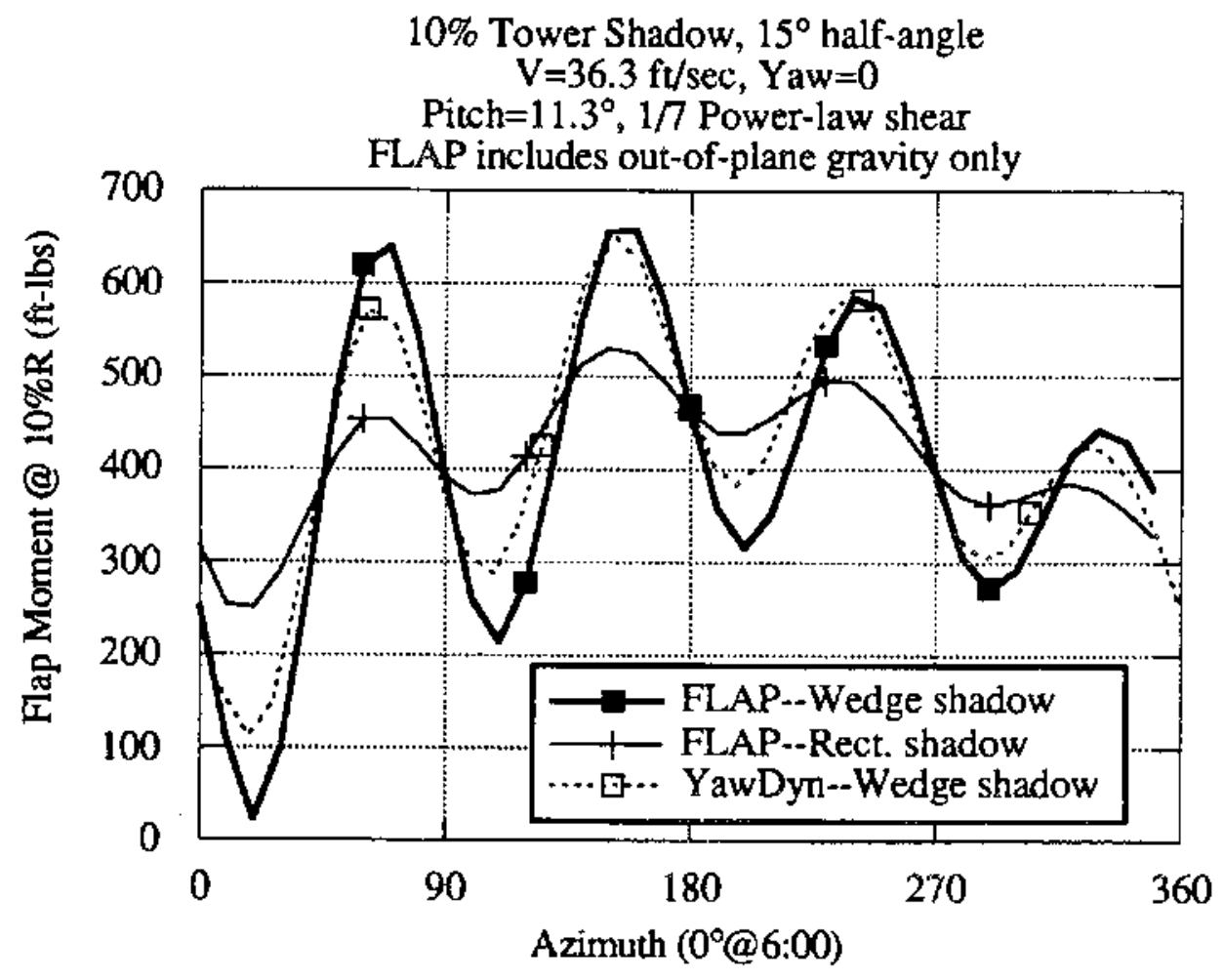

Figure 5.3 Comparison of YawDyn and FLAP predictions for a simple wind shear and tower shadow flow. All tower shadows have the same centerline deficit and the same width at the $75 \%$ span station.

Next, look at a comparison of test data and YawDyn and FLAP predictions. Figure 5.4 shows the flap moments from data set 901-3. This represents the best agreement found in any of the comparisons and shows that YawDyn can predict the flap loads with great accuracy. Figure 5.5 compares the same data set with YawDyn predictions when the dynamic stall option is turned off. It can be seen that there is a slight improvement in the agreement between data and predictions when dynamic stall is included. Figures 5.6 and 5.7 show similar comparisons for data set 901-1. Figure 5.6 shows the entire data set and makes it apparent that YawDyn generally predicts the mean flap load and the cyclic content. But there are a number of instances where the measured and predicted loads differ greatly. Figure 5.7 shows the first three seconds of the same data set to concentrate on the cyclic content. Again, the agreement is quite good but not complete. 


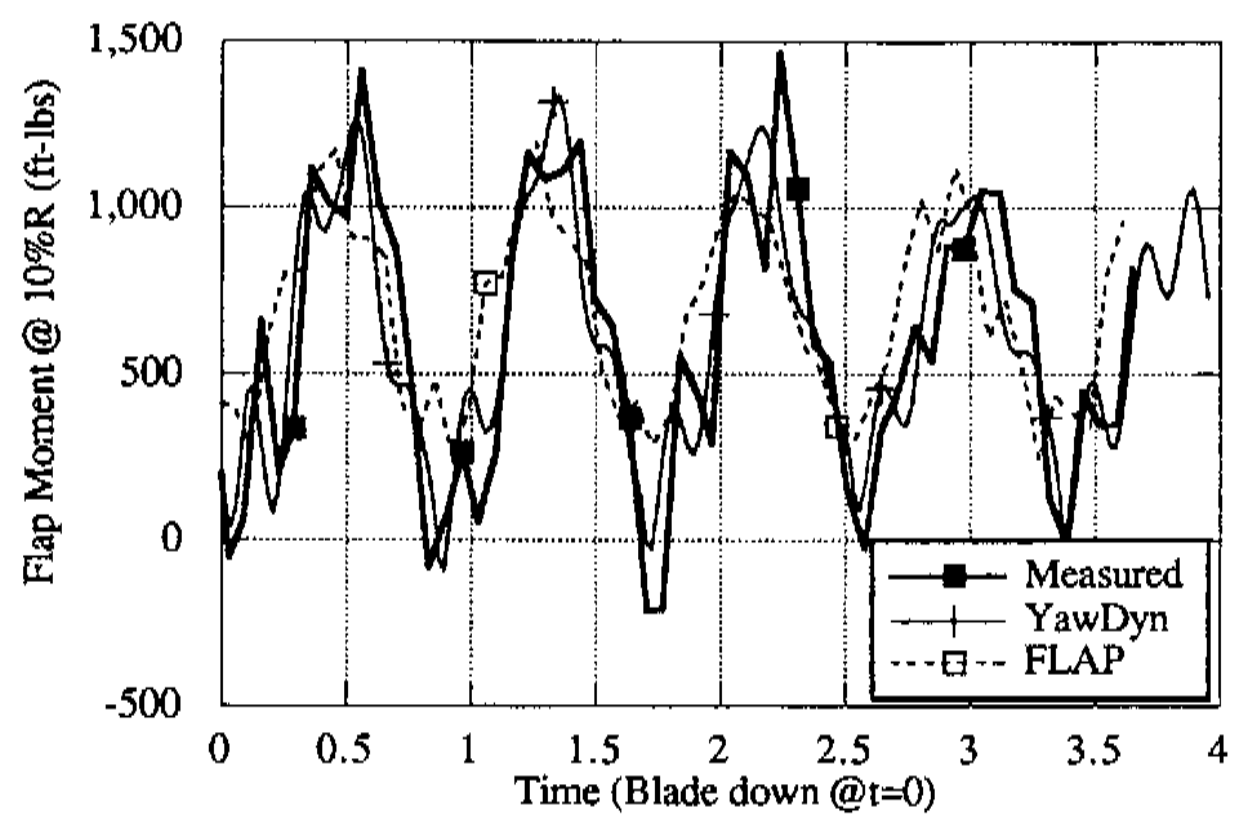

Figure 5.4 Comparison of flap moments for the Combined Experiment data set 901-3.

----a-- YawDyn (with dyn. stall) - - - - YawDyn (no dyn. stall) $\longrightarrow$ Measured

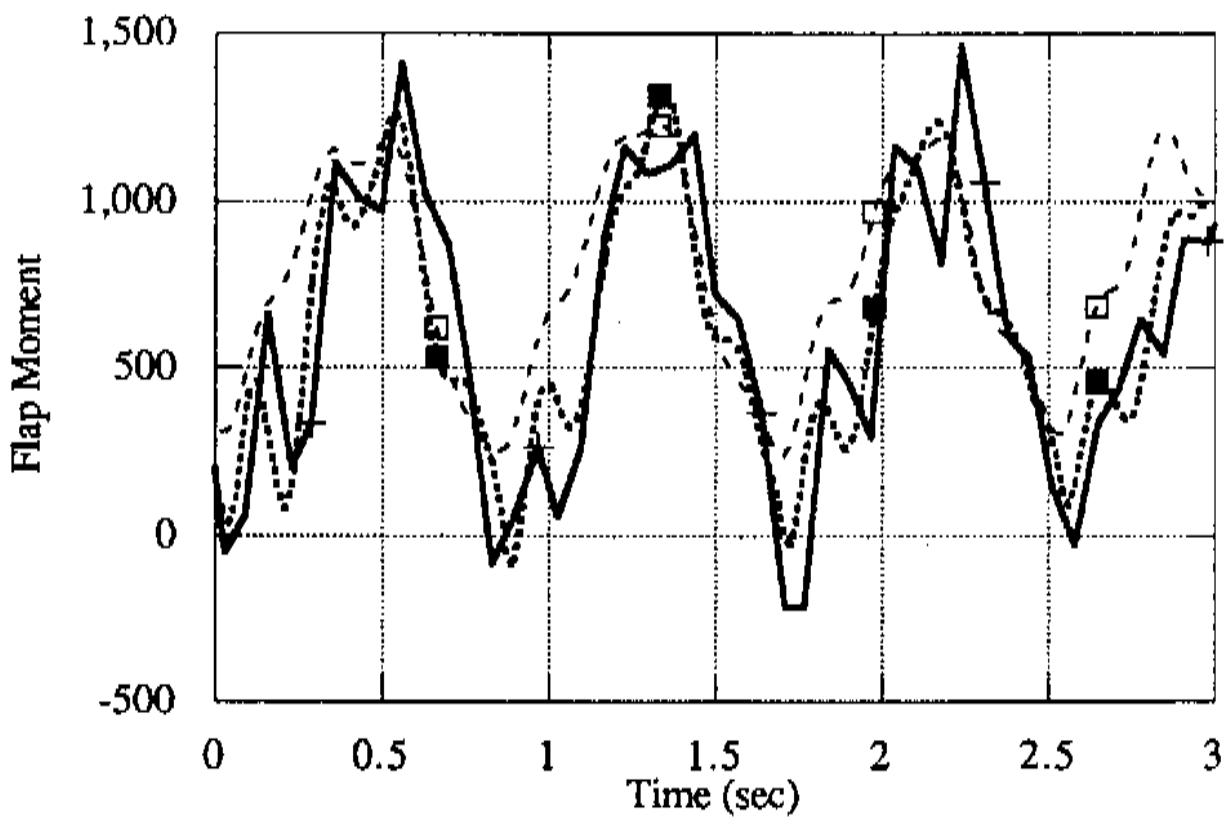

Figure 5.5 Including dynamic stall in the YawDyn predictions makes a slight improvement in the accuracy of the cyclic loads. The data are the same as presented in Figure 5.4. 


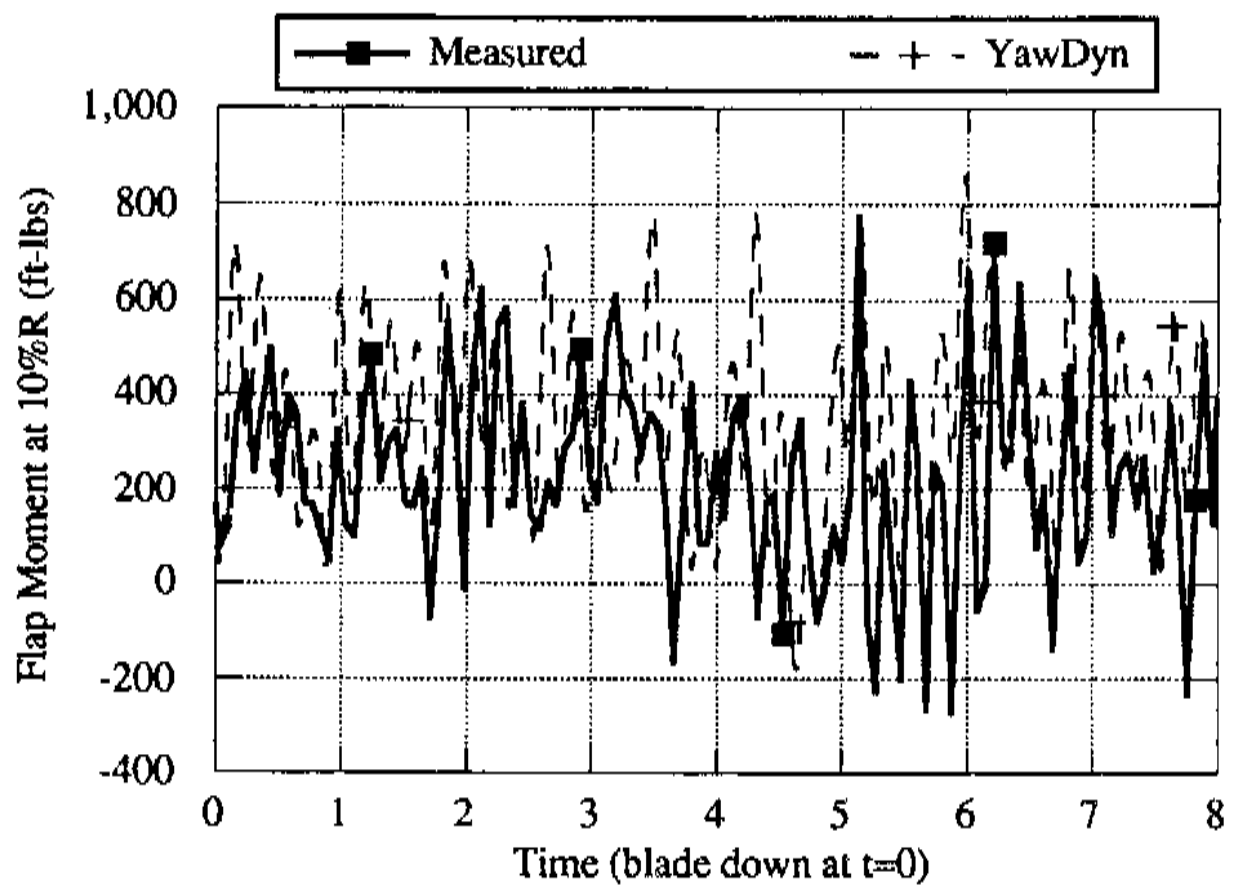

Figure 5.6 Comparison of measured and predicted flap moments for Combined Experiment data set $901-1$

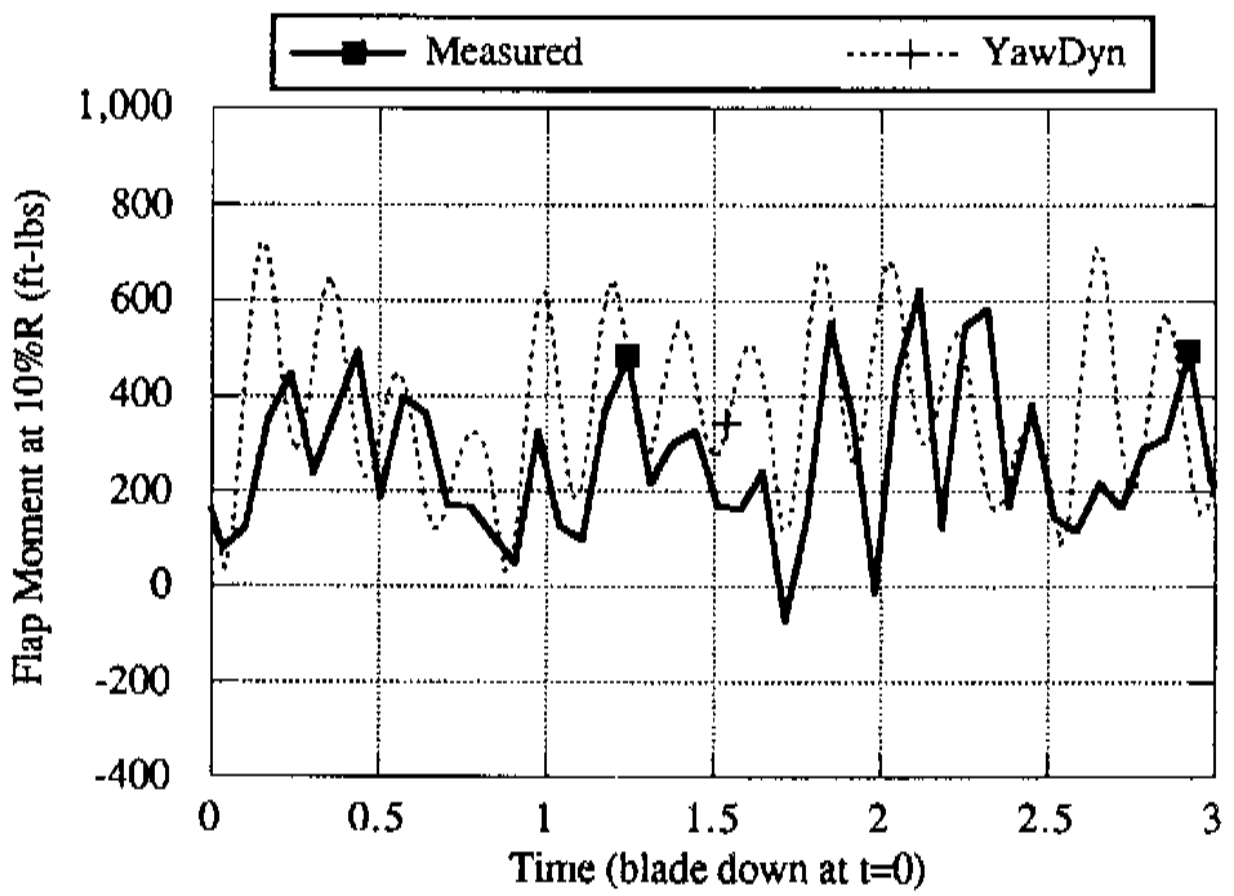

Figure 5.7 An expanded view of Figure 5.6, concentrating on the first three seconds of the test. 


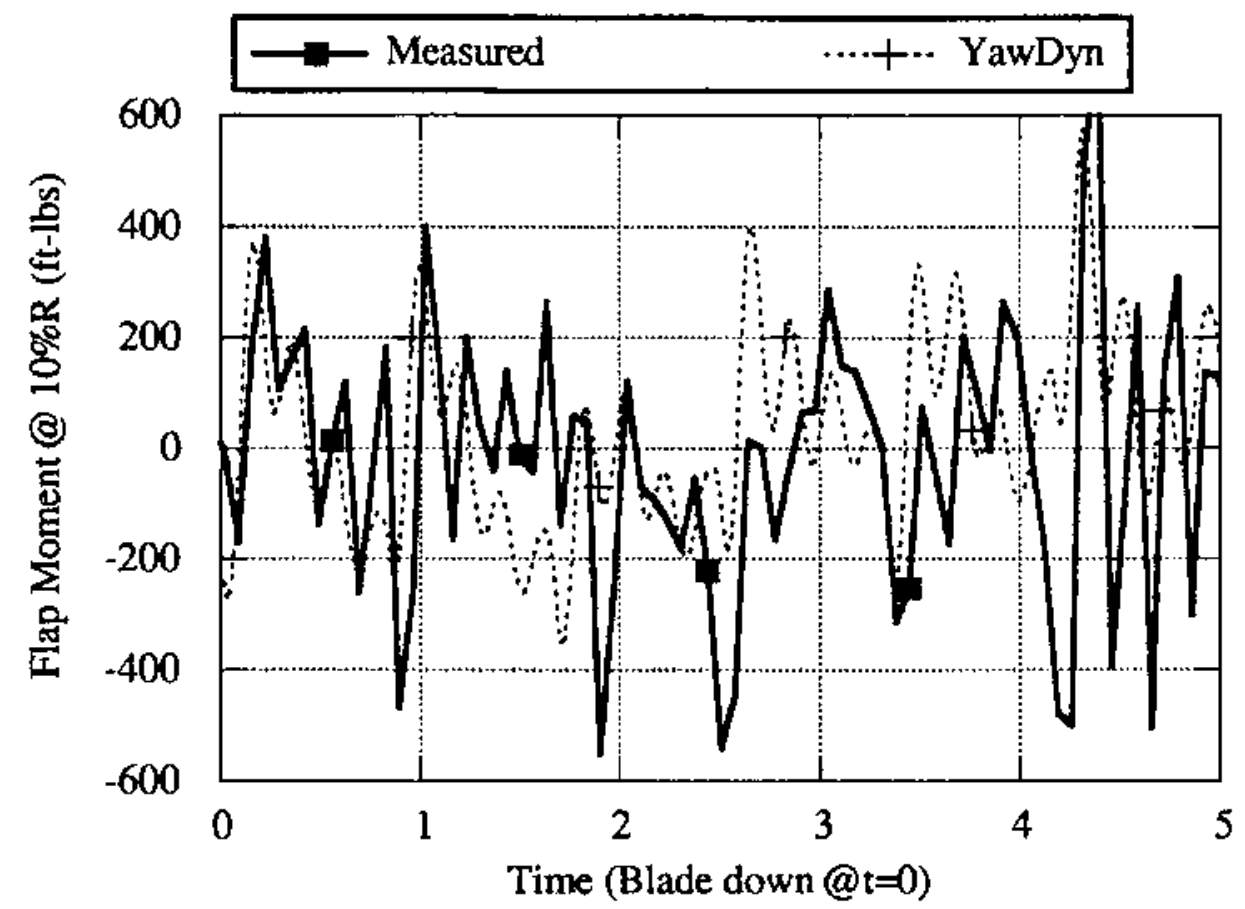

Figure 5.8 Comparison of flap moments from Combined Experiment data set 901-2.

Figure 5.8 shows the final comparison of measured and predicted flap moments. Again the trends are the same. The mean and lp cyclic moments are matched closely while the $4 \mathrm{p}$ cyclic shows greater discrepancies. This is believed to be due to the variability of the strength of the tower shadow in the actual test. In YawDyn the shadow is assumed to be of constant strength, location and width while in fact the tower wake must be meandering and may exhibit some periodic (vortex street) character.

From these three data sets one can conclude that the model is reasonably accurate in representing the flap behavior of the Combined Experiment rotor. It can also be concluded that dynamic stall has a slight effect on the flap moments and that including dynamic stall in the model improves the accuracy of the predictions. From a designer's point of view, it is likely that the model is more accurate than the knowledge of wind characteristics which must be entered into the model when seeking extreme load conditions.

Yaw Moment Predictions. The most important validation for the purposes of this project is of yaw moment predictions. Accurate prediction of yaw moments is necessary for design of yaw control systems. It is also important that the yaw loads be calculated correctly for accurate prediction of free-yaw motions. The same three data sets discussed above were used to examine the adequacy of the yaw load predictions. YawDyn was run with the same inputs as detailed above as well.

Figures 5.9 through 5.12 show comparisons of measured and calculated yaw moments for the three data sets. A $180 \mathrm{ft}-\mathrm{lb}, 1 \mathrm{p}$ cyclic yaw moment was added to all of the predictions to account for the known mass imbalance of the rotor. Since YawDyn cannot directly model the mass imbalance as it can the pitch imbalance, the lp load was added to the YawDyn outputs. Figures 5.9 and 5.10 show the same data, but Figure 
5.10 focuses on the first seconds of the simulation so that the cyclic content of the yaw moment can be more easily seen.

In all cases the mean and $1 p$ yaw moments show the best agreement while the $3 p$ cyclic moment shows less consistent agreement. As in the flap moment comparisons, the cyclic moment is over- and under-estimated at various times in the simulations. Since the $3 p$ load results primarily from $4 p$ flap oscillations, this result is expected and occurs for the same reason as the inconsistencies noted in the $4 \mathrm{p}$ flap moments.

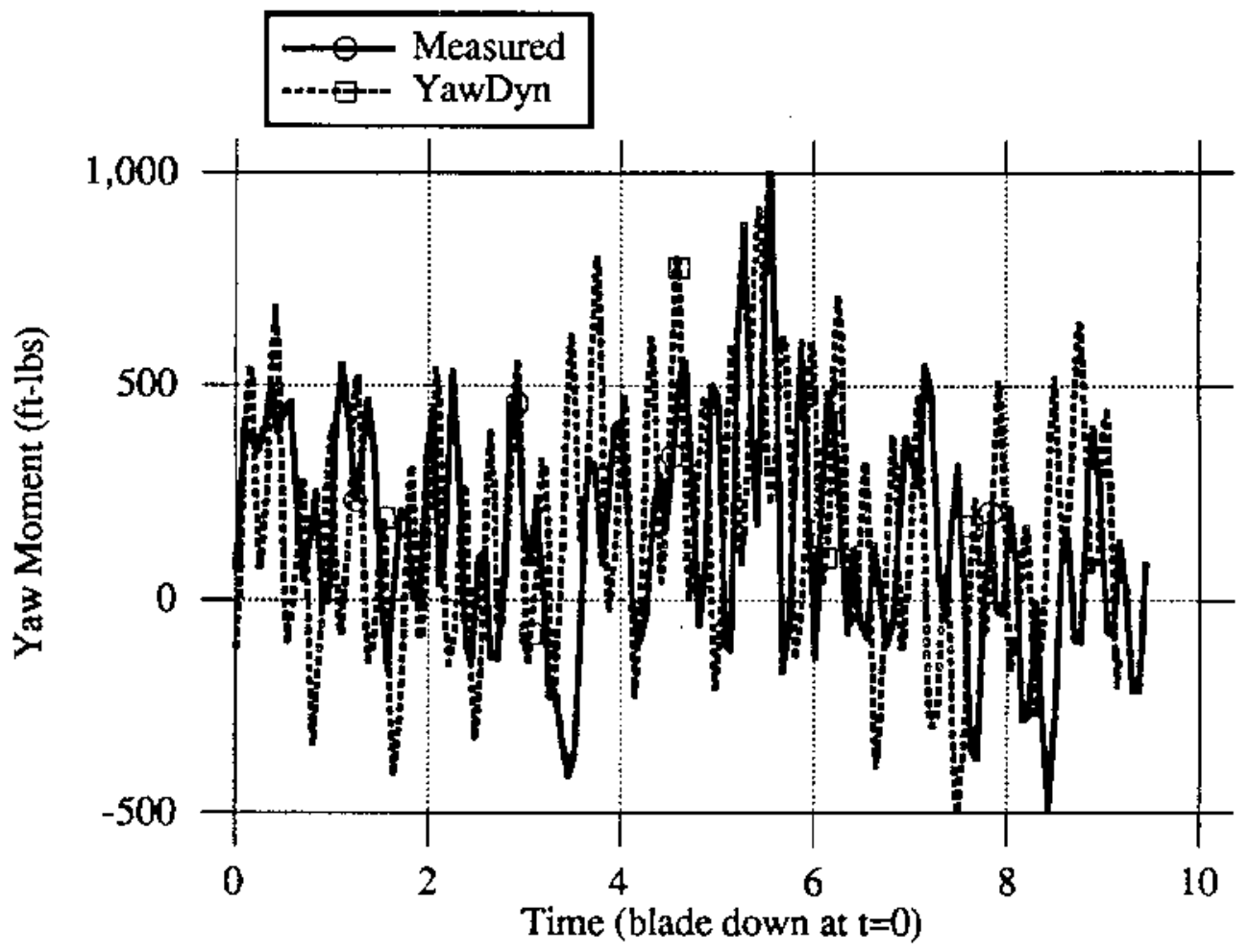

Figure 5.9 Comparison of predicted and measured yaw moments. YawDyn predictions include a $180 \mathrm{ft}-\mathrm{lb}, 1 \mathrm{p}$ moment due to mass imbalance. Combined Experiment data set CE901-1.

It will be shown in Section 6 that the dynamic stall and skewed wake correction have a very significant effect on the yaw moment predictions. Without these factors included in the analysis the agreement between measured and predicted yaw moments is much poorer. For example, Figure 5.13 shows the results from the CE901-3 data set (Figure 5.12) compared with the predictions when dynamic stall is not considered in the model. The curve labeled "YawDyn w/o DS" is the prediction with both Gormont parameters set to zero to entirely remove the stall hysteresis. It is clear that the mean yaw moment is greatly influenced and that the agreement is poorer than when the hysteresis is included. This data set had high wind speed and a large yaw angle resulting in high mean and cyclic angles of attack. Thus it is particularly affected by dynamic stall. But similar influences are seen, though to a lesser extent, in lower winds. 


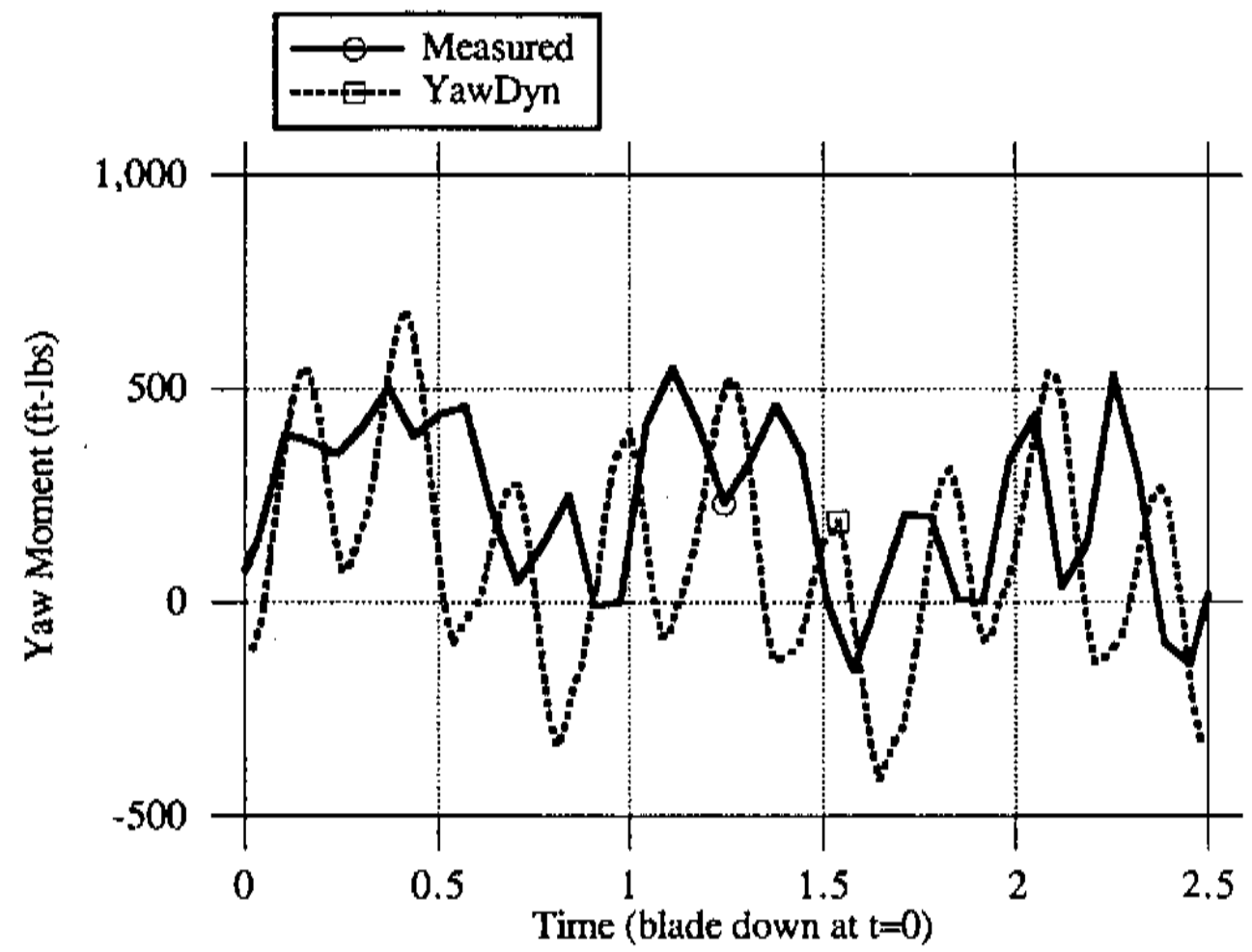

Figure 5.10 Expanded view of Figure 5.9 showing the first three revolutions of the rotor. Combined Experiment data set CE901-1.

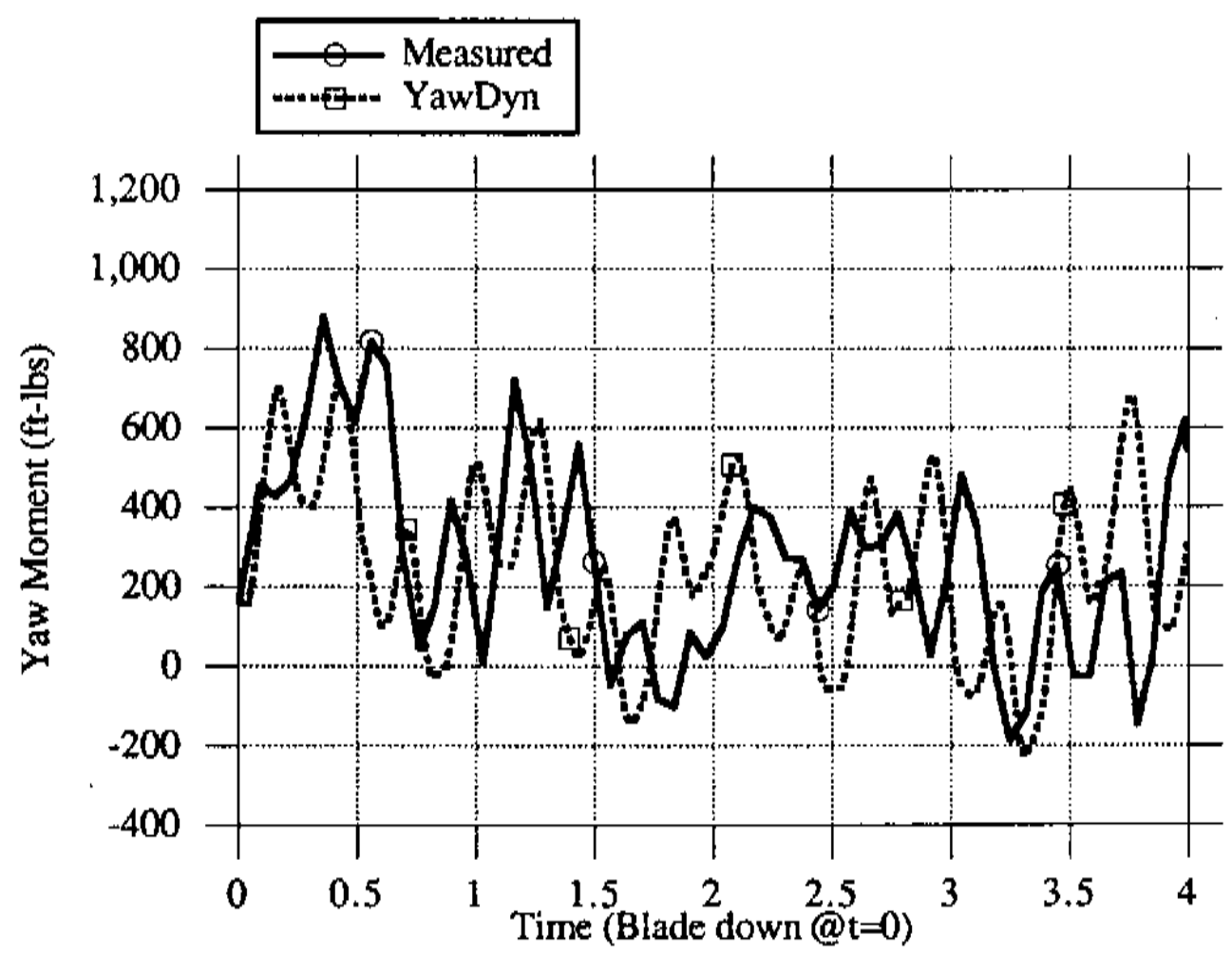

Figure 5.11 Comparison of predicted and measured yaw moments. YawDyn predictions include a $180 \mathrm{ft}-\mathrm{lb}, 1 \mathrm{p}$ moment due to mass imbalance. Combined Experiment data set CE901-2. 


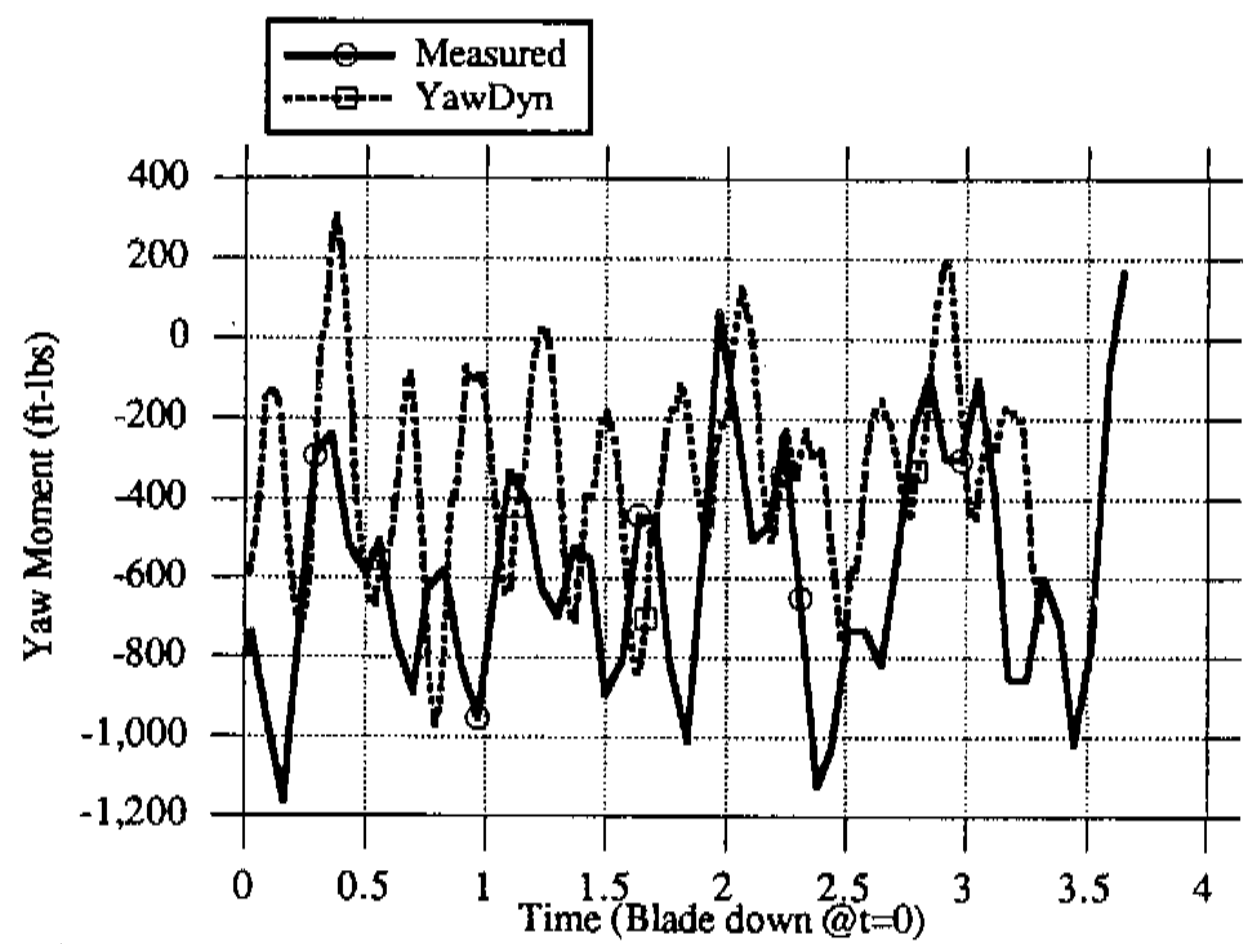

Figure 5.12 Comparison of predicted and measured yaw moments. YawDyn predictions include a $180 \mathrm{ft}-\mathrm{lb}, 1 \mathrm{p}$ moment due to mass imbalance. Combined Experiment data set CE901-3.

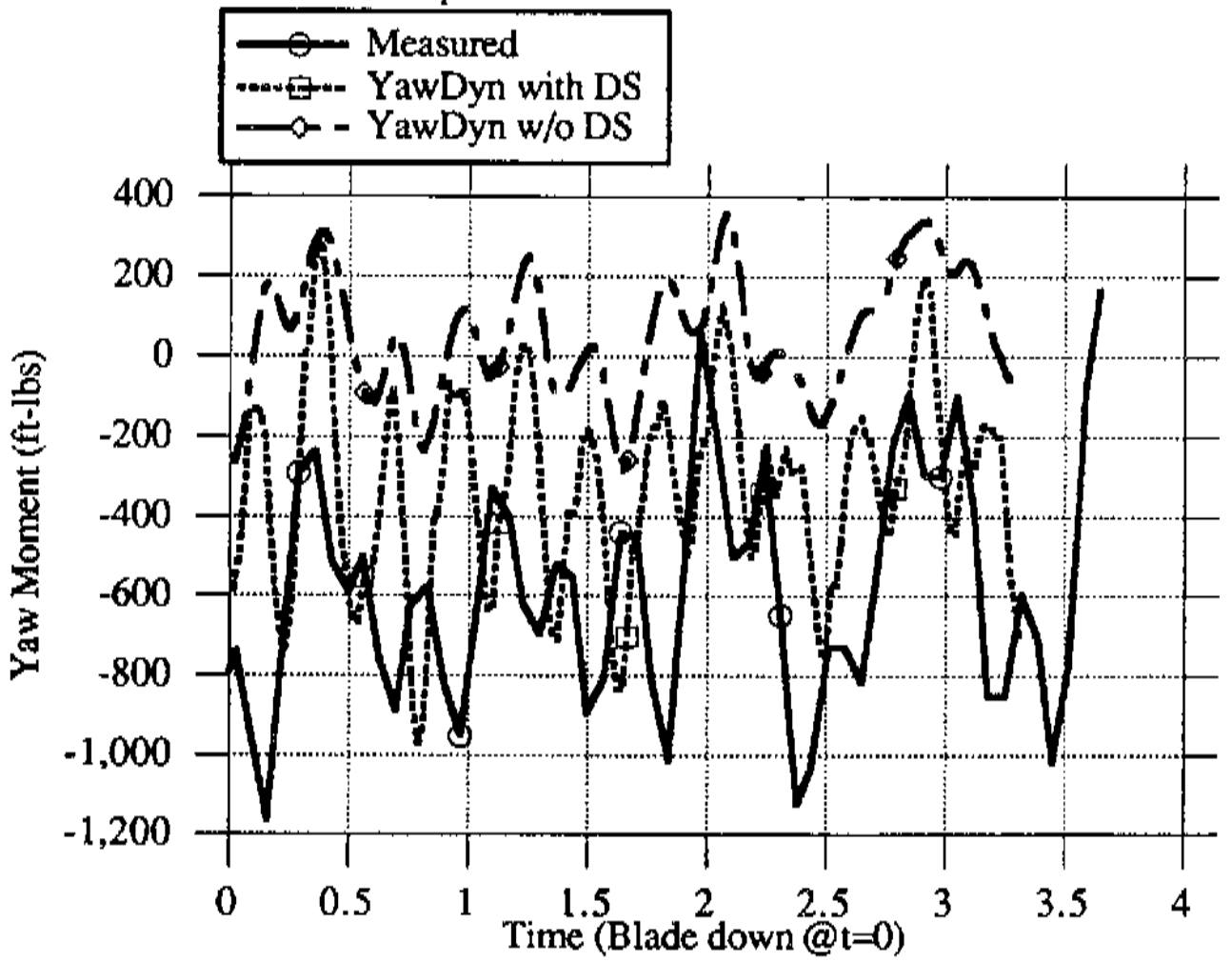

Figure 5.13 Comparison of predicted and measured yaw moments showing the influence of stall hysteresis. YawDyn predictions include a $180 \mathrm{ft}-1 \mathrm{~b}, 1 \mathrm{p}$ moment due to mass imbalance. Combined Experiment data set CE901-3. 
Dynamic Stall Model. A final point regarding the Combined Experiment validation can be made by examining the lift coefficient and angle of attack as measured and predicted. First notice in Figure 5.14 that the angle of attack varies in a cyclic manner, primarily at $1 \mathrm{p}$. The dominant cause of this $1 \mathrm{p}$ variation is the advancing and retreating blade while the rotor is yawed. There is also a brief, sharp dip in the angle of attack as the blade passes through the tower shadow. This is observed as a brief (approximately 100-200 $\mathrm{ms}$ ) episode as the angle reaches the maximum value of each revolution. Both the test data and the predictions display this same character, though it is much less regular in the test data.

Figure 5.15 demonstrates clearly that there is stall hysteresis on this blade. The measurements and predictions both demonstrate the hysteresis, though again the measurements show considerably more variability in the values. As mentioned carlier, this hysteresis results in asymmetry of the lift coefficient from one side of the rotor to the other. This is the cause of the high mean yaw moments observed in the predictions and measurements.

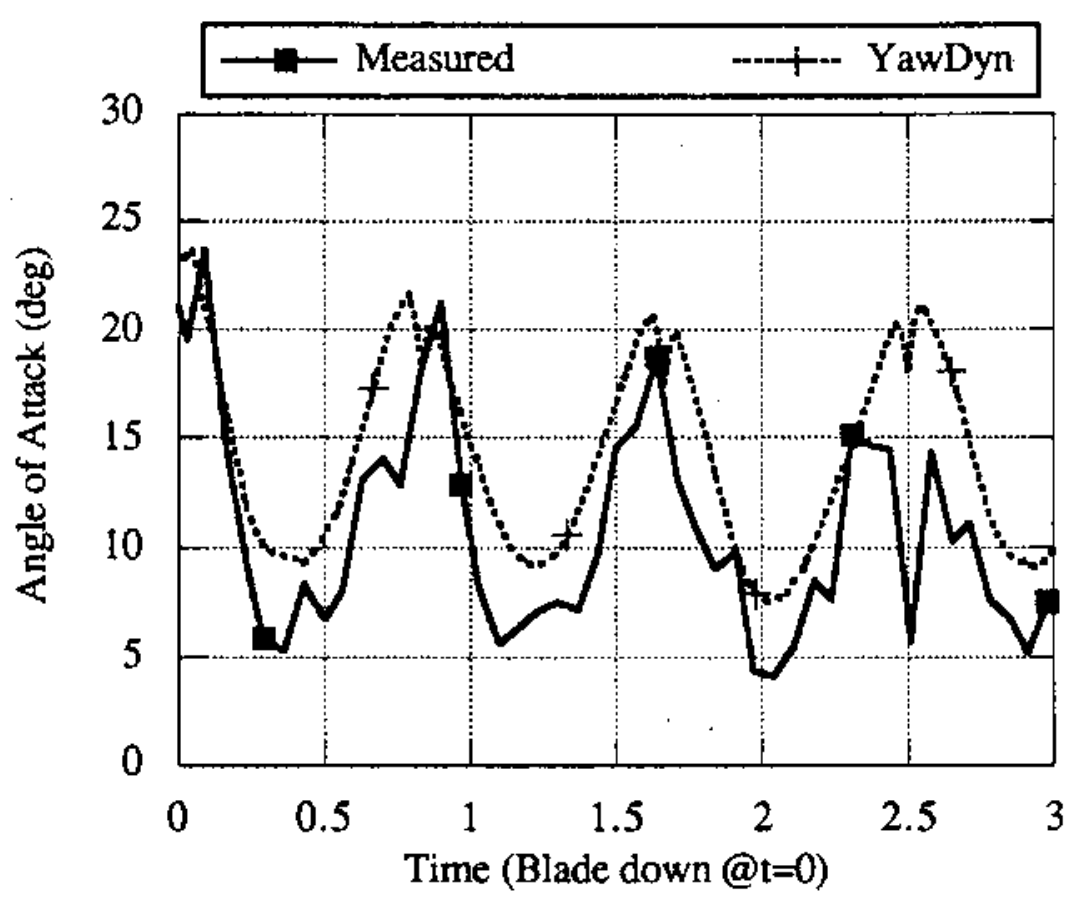

Figure 5.14 Angle of attack time history for data set 901-3. Note the characteristic 1p variation with a sharp dip caused by tower shadow. 


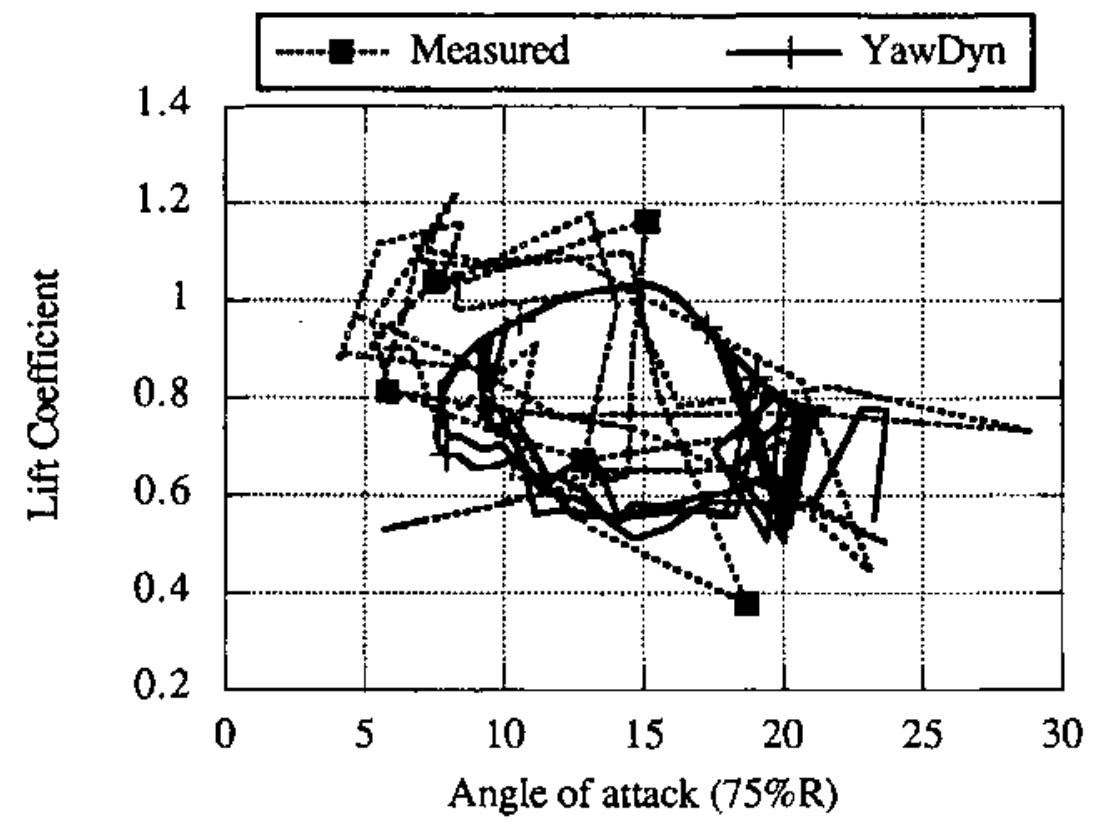

Figure 5.15 Comparison of stall hysteresis as measured at the $80 \%$ station and predicted at the $75 \%$ station for data set $901-3$.

\subsection{Free-yaw predictions and measurements from the Combined Experiment rotor}

The YawDyn program is capable of predicting yaw motions as well as yaw loads. There is little data available with sufficient resolution of the approach wind (including wind shears and vertical wind) for validation of the calculations. However, one data set was obtained from the SERI Combined Experiment. The rotor was held at a fixed yaw angle, $-32^{\circ}$ from the wind direction. It was then released (at time $=0$ ) to yaw freely for a short period before the yaw brake was once again applied. Measurements of the horizontal wind approaching the rotor were made with a vertical-plane array of vanetype anemometers. The measured wind speed, wind direction, horizontal shear and vertical shear were input to YawDyn as a function of time to simulate the same test conditions. The vertical component of wind was not available and was assumed zero.

The predicted yaw motion is compared with the measured motion in Figure 5.16 below. Both the predictions and measurements show a slight overshoot in the yaw response. The general shapes of the two curves are very similar, with both showing that the rotor does not return to zero yaw angle. The predicted yaw rate (and hence the time required to reach a yaw angle of zero) is faster than the measured value.

The predictions were made assuming a constant yaw friction of $75 \mathrm{ft}-\mathrm{lbs}$ and zero mechanical yaw damping. These values were arbitrarily selected since the actual values were not available. Lack of measured values limits the ability to completely assess the accuracy of the prediction method. The mass moment of inertia of the system about the yaw axis was estimated from the weights and locations of major elements. There is an uncertainiy of approximately $25 \%$ in this value. However, increasing the yaw inertia by $25 \%$ is not sufficient to achieve a match between the predicted and measured time to reach zero yaw angle. 
This comparison and the results of sensitivity studies lead to the conclusion that the yaw motion predictions are reasonable but of unknown absolute accuracy.

\subsection{Teeter Predictions by YawDyn_and_STRAP}

Near the end of the research project, the YawDyn program was modified to permit analysis of the teetering rotor. In this section the program is compared with the STRAP code (the SERI Teetering Rotor Analysis Program, a derivative of the FLAP code). This was done to verify basic operation of the YawDyn teetering option and should not be considered a thorough validation of the program or its underlying assumptions. Considerable work must be done in the future to determine the most useful method for analyzing the teetering rotor.

Table 5.4 lists a number of model inputs for the comparisons that follow. Though at first glance the inputs may appear inconsistent, they were selected to match the location of the rotor center of gravity relative to the teeter axis. To minimize differences in the approaches of YawDyn and STRAP, both were used to analyze an idealized rotor similar to the ESI-80. Since STRAP employs a linear aerodynamics model, both programs were given inputs to create a linear lift curve over the full range of angle of attack $\left( \pm 180^{\circ}\right)$. The drag coefficient was set to zero to eliminate differences in the approach to modeling the drag behavior. This is clearly not realistic but does ensure that differences in the predictions of the code are not due to differences in the airfoil model. The yaw angle was set to zero to eliminate effects due to skewed wake corrections. Dynamic stall, vertical component of wind speed and horizontal shear are not available in STRAP and were therefore not used in YawDyn. Delta-three is not available in the YawDyn model, necessitating use of $\delta_{3}=0$ in STRAP.

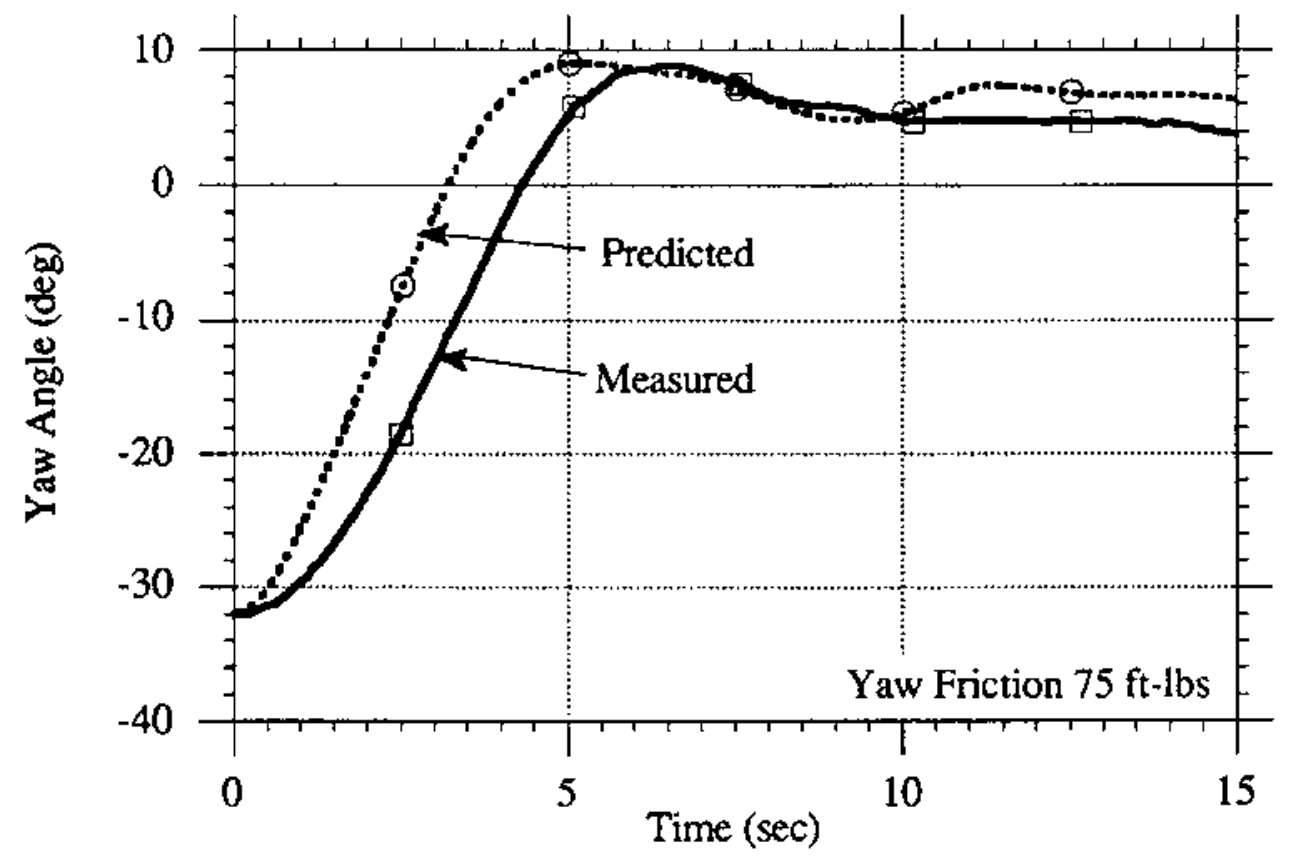

Figure 5.16 Comparison of predicted and measured free-yaw response of the Combined Experiment rotor. At time $=0$ the rotor was released from rest at a yaw angle of $-32^{\circ}$. Data set number GS144. 
Table 5.4 Parameters used in the YawDyn and STRAP analyses of the teetering rotor.

\begin{tabular}{|l|l|l|}
\hline \multicolumn{1}{|c|}{ Parameter } & \multicolumn{1}{|c|}{ YawDyn Value } & \multicolumn{1}{c|}{ STRAP Value } \\
\hline Yaw angle & $0^{\circ}$ & $0^{\circ}$ \\
\hline Flap DOF? & Rigid tecter & One mode (rigid teeter) \\
\hline Hub & Teetering, $\delta 3=0$ & Teetering, $\delta 3=0$ \\
\hline Pitch angles (all blades) & $0^{\circ}$ & $0^{\circ}$ \\
\hline Dynamic stall & None $(\mathrm{A}=0.0 . \mathrm{B}=0.0)$ & NA \\
\hline Number of sectors & 200 & NA \\
\hline Convergence tolerance & $0.01^{\circ}$ & $2 \%$ \\
\hline Horizontal shear & 0.0 & 0.0 \\
\hline Vertical wind component & 0.0 & 0.0 \\
\hline Tower shadow & None & None \\
\hline Blade mass (slugs) & 55.7 & 26.71 \\
\hline Hub mass (slugs) & 0 & 55.9 \\
\hline Undersling (feet) & 0.28 & 0.75 \\
\hline Hub distance (feet) & NA & 0.24 \\
\hline Lift curve slope & 5.2 & 5.2 \\
\hline Drag coefficient & 0.0 & 0.0 \\
\hline
\end{tabular}

- Yawdyn, 0 Coning, $0 \mathrm{shr} \longrightarrow$ Strap, 0 Coning, $0 \mathrm{shr}$ ....-... Yawdyn, 0 Coning, .14 shr ...-*... Strap, 0 Coning, $.14 \mathrm{shr}$ $\multimap$ - YawDyn, $7^{\circ}$ Coning, $14 \mathrm{shr} \longrightarrow *-$ Strap, $7^{\circ}$ Coning, .14 shr

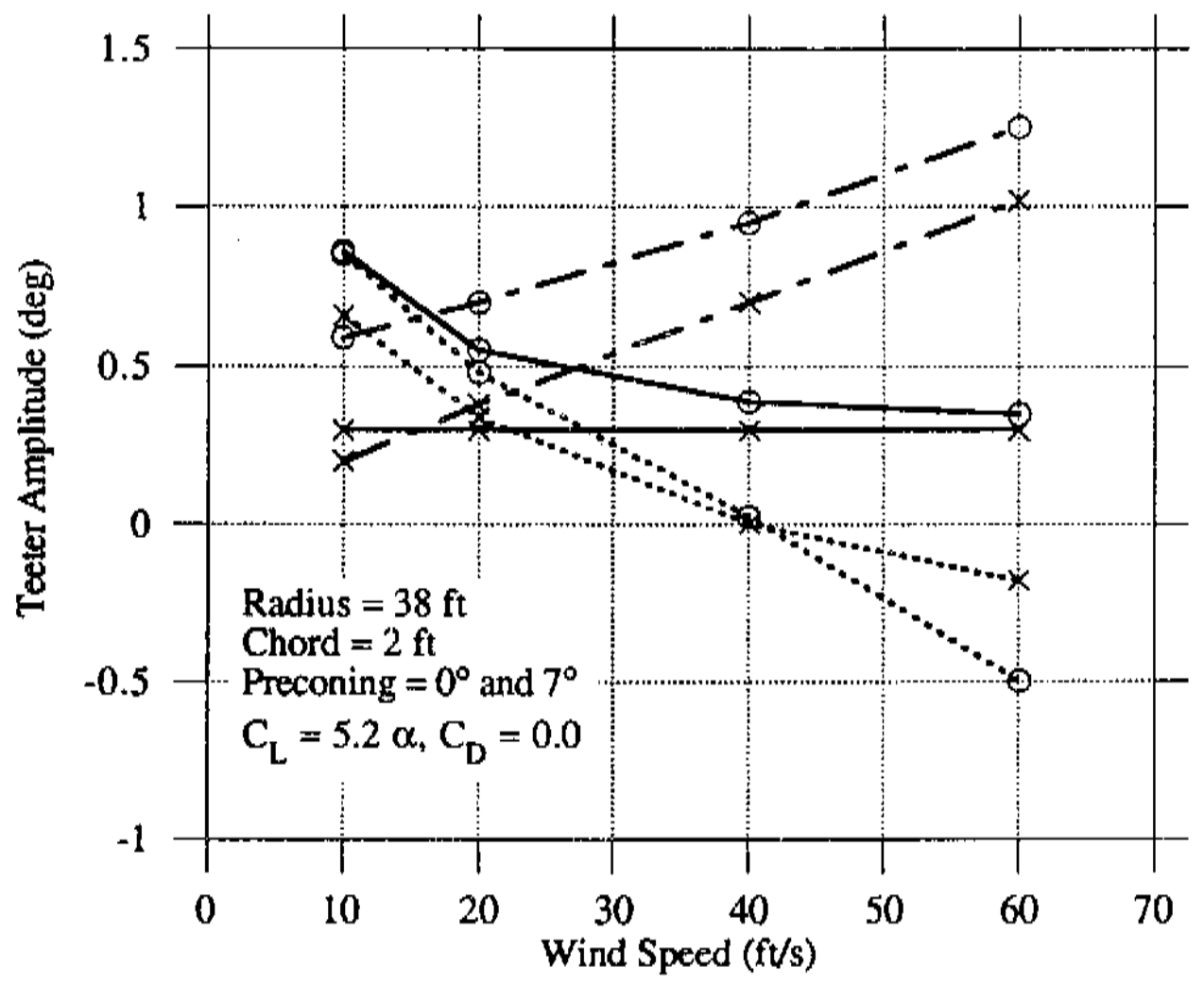

Figure 5.17 Teeter amplitudes predicted by YawDyn and STRAP. 
With these restrictions and conditions, the excitation of teeter motion was due to undersling or vertical wind shear. Both were investigated with the results shown in Figure 5.17. In all cases shown the rotor mass of fset from the teeter axis caused teetering motion. Four of the curves also show the effect of $1 / 7$ power-law vertical wind shear $(.14 \mathrm{shr})$. The two programs produce the same trend when there is vertical wind shear. With no coning and no shear, STRAP predicts a teeter amplitude independedent of wind speed while YawDyn predicts decreasing teeter amplitude as wind speed increases. This is a result of differences used in the two programs to estimate induced velocities. YawDyn iterates to find a solution to the full, nonlinear equation for the induction factor. STRAP uses small angle assumptions in conjunction with a linear lift curve to develop an approximate, explicit equation for the induced velocity. This leads to significantly reduced computation time, since iteration is not required, but also yields a result which is less accurate. The linearized, analytic solution of Stoddard [Stoddard, 1978; Stoddard, 1988] gives the same result as STRAP in the simple case where undersling is the only teeter forcing function. Since YawDyn is solving the more exact equation, it is expected that it yields a more accurate result. The two programs give the same prediction at the windspeed where they have the same induced velocity.

There is one other potentially important difference between the aerodynamic analyses of YawDyn and STRAP. YawDyn assumes the wake is always in equilibrium (quasisteady flow). STRAP assumes the wake is frozen. Thus YawDyn includes the effect of the blade flap velocity in the estimation of the induced velocity while STRAP does not. It is unclear from these simple validation tests how much this difference in assumptions may contribute to the differences in the program predictions.

From this simple validation test one can conclude that YawDyn is producing reasonable results and contains no programming errors which give results inconsistent with the underlying equations and assumptions. Much more work is required before the method can be proven to yield results which are accurate in an absolute sense. 


\subsection{Sensitivity Studies}

\subsection{Introduction}

One of the primary objectives of this research was to determine factors which have the greatest influence on the yaw loads and motions of the horizontal axis wind turbine. To accomplish this objective YawDyn was run for a wide variety of wind, machine and model parameters. Then the changes in predicted mean and cyclic loads were examined to determine which loads were most affected by the various inputs and assumptions. This process was performed continuously throughout the project for a number of rigidhub rotors. There were far too many conditions and assumptions to present in this report. A number of the findings were presented in annual progress reports and papers. In this report the results of a final series of sensitivity studies will be presented. This series was selected to highlight the most important findings from the past years' work.

All comparisons were done in a manner which was as self-consistent as possible. First a baseline rotor and operating condition was selected. The SERI Combined Experiment rotor was selected for the rigid hub studies and the ESI-80 was selected for the teetering hub cases. The baseline conditions used the model with the most accurate assumptions (e.g. dynamic stall, skewed wake corrections and the flap DOF were included) and typical wind conditions. Then a series of calculations were performed where only one parameter at a time was changed to observe the importance of that parameter.

The magnitude of change for each parameter was subjectively selected to represent a comparable and "significant" change. This rather arbitrary method was employed with the goal of identifying the important parameters in a realistic and practical manner. Other methods which are more quantitative, such as selecting a fixed percentage change in each parameter, were felt to be less useful. For instance, a $50 \%$ change in the blade stiffness would be a much more dramatic change (and more difficult to execute in the design) than a 50\% change in the vertical wind shear. And many of the parameters have a baseline value of zero, making percent changes meaningless.

It is important to note that the sensitivity results presented in this report are felt to be generally valid and applicable to a variety of wind turbines. It is clear, however, that there will be many exceptions to the general observations noted below. These comparisons are for illustrative purposes only and should not be applied literally to any other wind turbine. The only way to draw conclusions about a particular configuration is to exercise similar sensitivity studies for that turbine.

\subsection{Rigid-hub configuration}

The SERI Combined Experiment rotor was selected as the configuration for the rigidhub sensitivity studies. This was done because there is excellent data available for the rotor and airfoil and it is a configuration typical of many others. The general characteristics of the machine are presented in Appendix B. The rotor has some characteristics which are particularly important in the present study. The natural frequency of the blade in the first rotating flap mode is $4.06 \mathrm{p}$. This proximity to a rotor harmonic results in substantial flap response at $4 \mathrm{p}$. The $4 \mathrm{p}$ blade flap generates a large $3 p$ yaw moment (see section 3.2). Other rotors will generally not exhibit this large $4 p$ flap response. Also, the blades are untwisted. Thus the stall boundary moves slowly 
out along the blade as the wind speed increases rather than abruptly stalling over much of the span as it might if the blade were twisted. This means that other rotors may see more dramatic changes in loads near the stall boundary as wind or rotor conditions are varied.

Prior to conducting the sensitivity studies a number of calculations were performed to establish the basic performance of the system. The rotor was analyzed using YawDyn with all options "on" to provide the most realistic analysis. The wind speed was varied for a zero yaw angle and the yaw angle was varied for a fixed wind speed to see how the global characteristics such as power output and mean loads would change. The results are summarized in the figures below. In these figures the operating conditions are as noted in Table 6.1 except as noted on the figures.

Figure 6.1 shows the variation of mean rotor power and flap moments as a function of yaw angle. The plot uses the Cosine of the yaw angle for the $\mathrm{x}$-axis and shows leastsquares curve fits to illustrate that both the power and flap moment vary approximately as the first power of the cosine of the yaw angle. The power and loads are not identical for positive and negative yaw angles because the $1 / 7$ power-law vertical wind shear and tower shadow (with blade flexibility) introduce a slight asymmetry.

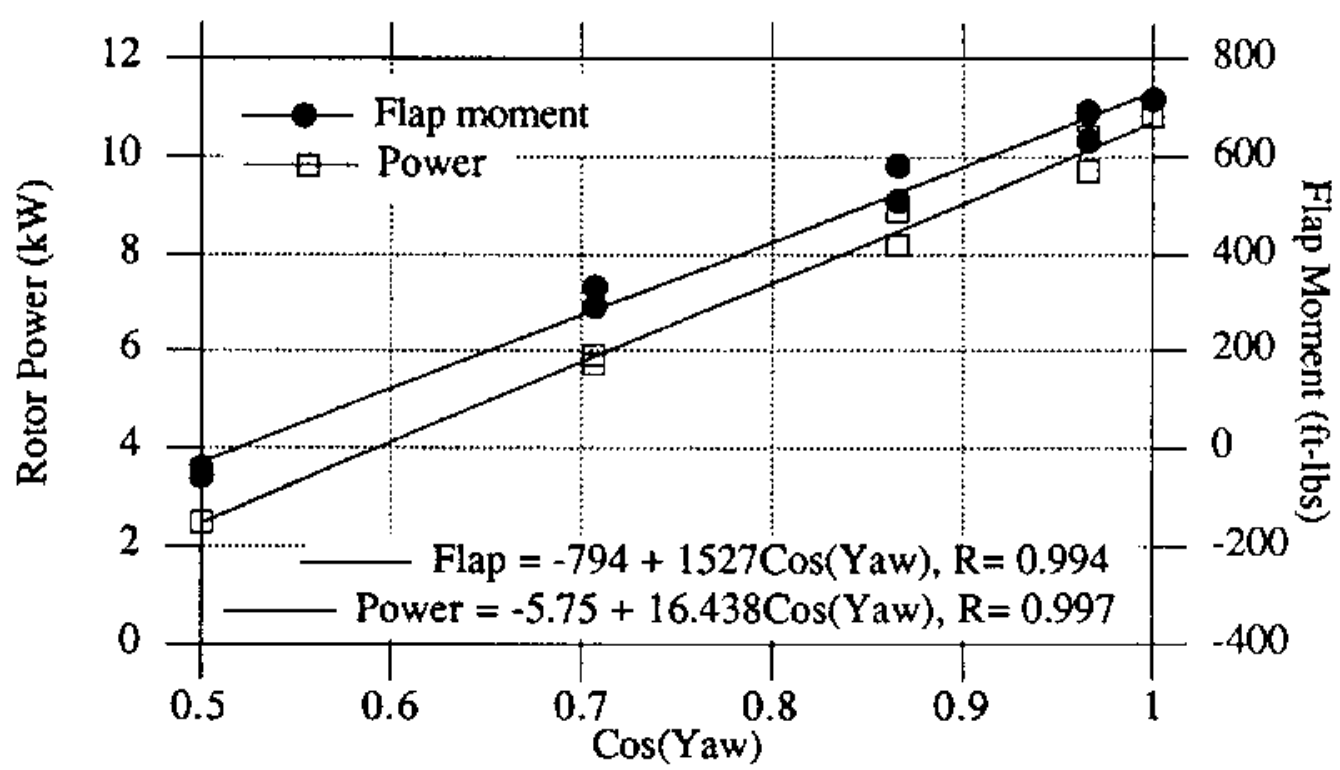

Figure 6.1 Variation of the predicted power output and mean flap moment of the Combined Experiment rotor. Wind speed $37 \mathrm{ft} / \mathrm{s}$, yaw angles from $+60^{\circ}$ to $-60^{\circ}$.

Figure 6.2 shows the variation of mean yaw moment with yaw angle for a wind speed of $37 \mathrm{ft} / \mathrm{sec}$. Note the yaw moment depends nearly linearly upon the yaw angle over a wide range of angles. It is also important to note the yaw moment is not zero at zero yaw angle. This means the rotor will tend to operate in a steady-state at a small (approximately $+3^{\circ}$ ) yaw error. This offset is caused by vertical wind shear and tower shadow and will depend upon the wind speed. The yaw moment is positive when the yaw angle is negative (and vice versa), except at very small angles. Thus the moment acts to reduce the yaw angle. This means the rotor will operate in a statically stable manner in downwind, free-yaw motion. 


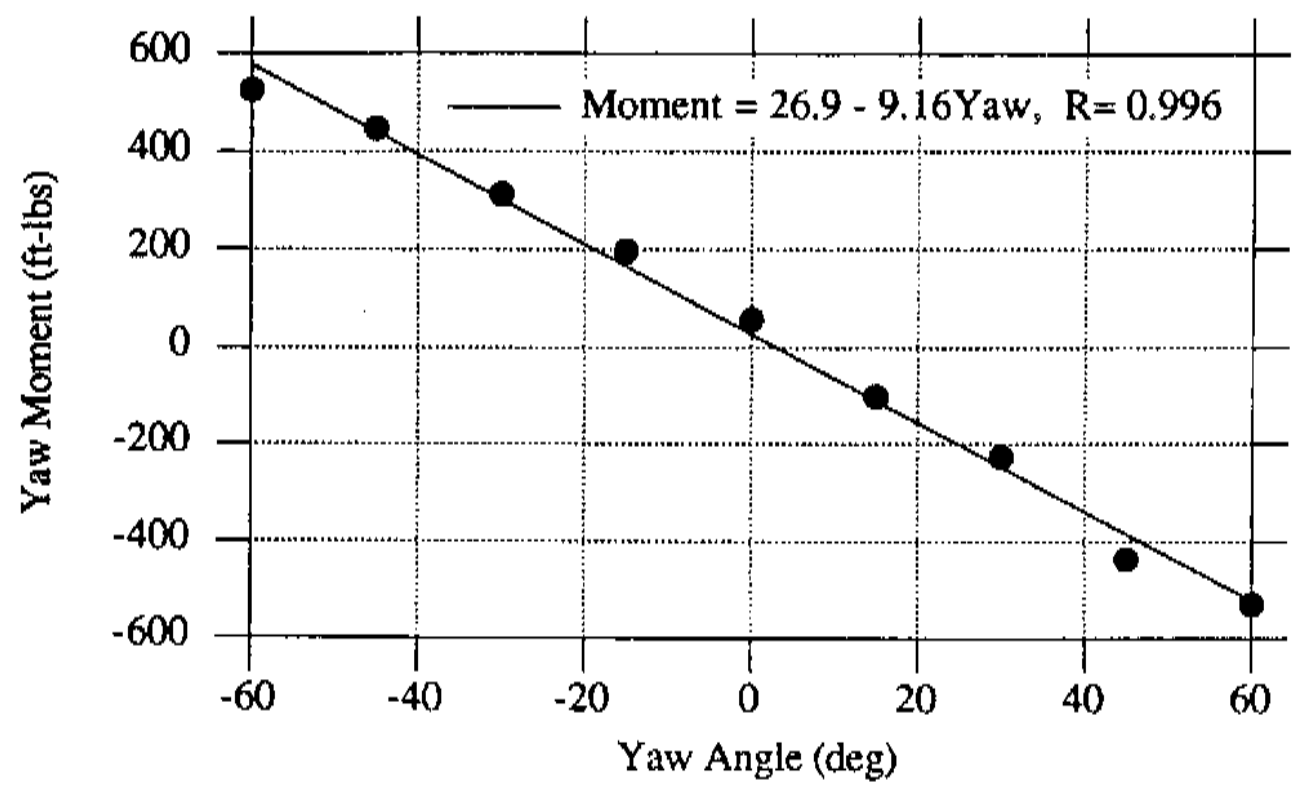

Figure 6.2 Variation of the predicted mean yaw moment of the Combined Experiment rotor. Wind speed $37 \mathrm{ft} / \mathrm{s}$, yaw angles from $+60^{\circ}$ to $-60^{\circ}$.

Figure 6.3 illustrates the variation of the mean loads with changes in wind speed. Figure 6.4 shows the variation in the mean angle of attack at the $75 \%$ radius and the mean rotor power for the same conditions.

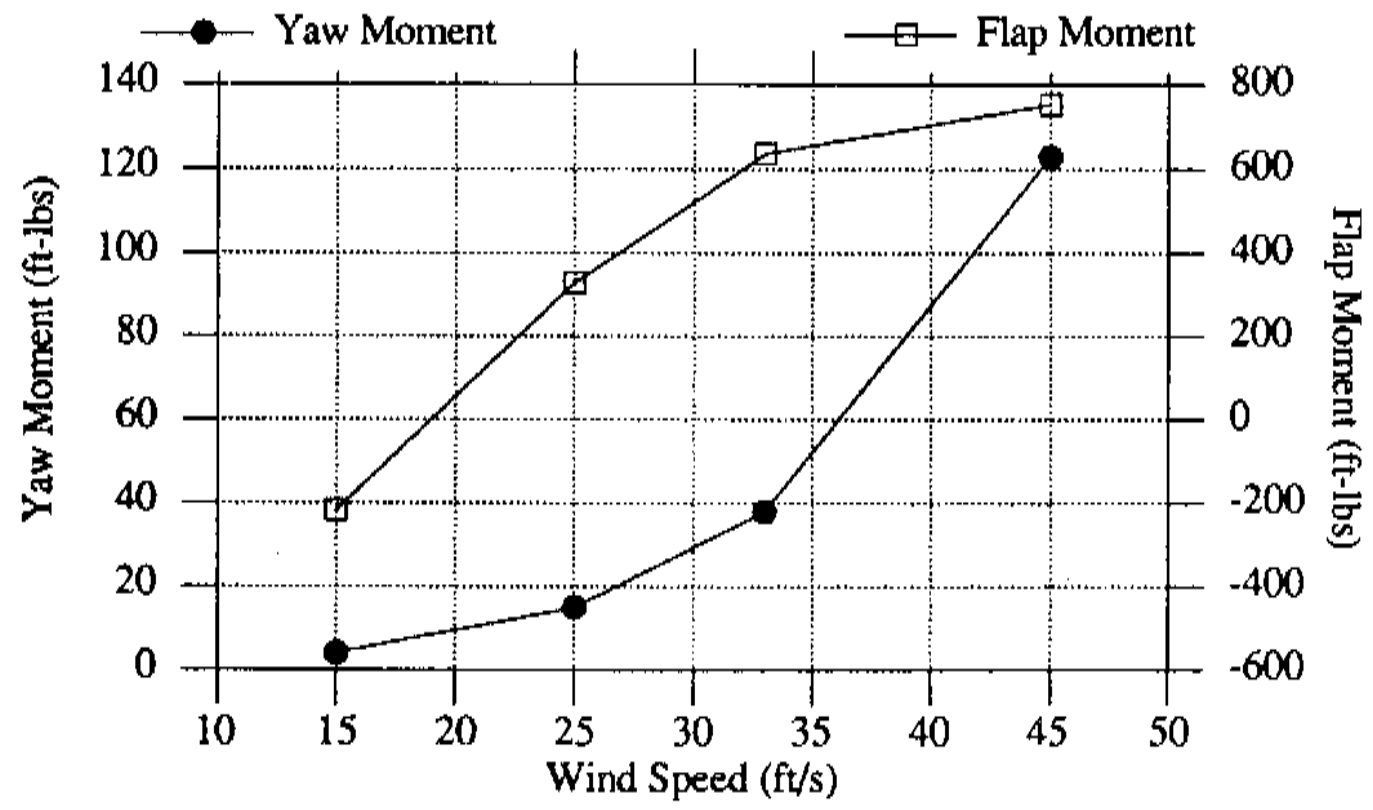

Figure 6.3 Variation of the predicted mean flap and yaw moments of the Combined Experiment rotor with hub-height wind speed. Yaw angle $0^{\circ}$. 


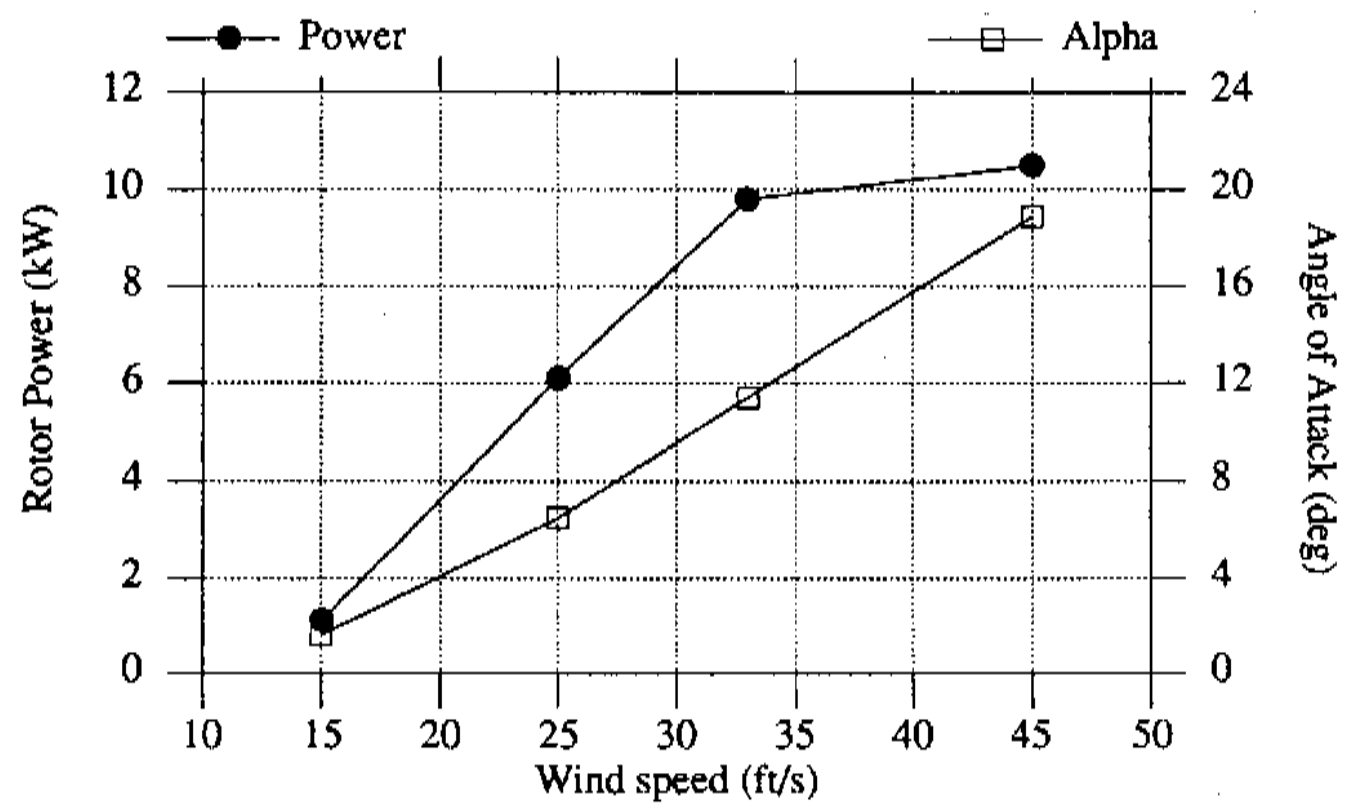

Figure 6.4 Variation of the predicted mean rotor power and angle of attack at the $75 \%$ station of the Combined Experiment rotor. Yaw angle $0^{\circ}$.

The results depicted in Figures 6.1 through 6.4 led to selection of a wind speed of 37 $\mathrm{ft} / \mathrm{sec}$ and a yaw angle of $-30^{\circ}$ for the sensitivity studies. At these conditions the $75 \%$ station is near the stall angle on average and moving in and out of stall as the blade rotates. This makes it possible to observe the effects of stall parameters in the modeling. To assist the reader in understanding the results of the sensitivity studies some figures are provided to depict the state of the rotor while operating in the baseline configuration.

Figure 6.5 shows the variation in angle of attack at the $75 \%$ station for the baseline conditions listed in Table 6.1. Note the blade is moving in and out of stall as the yaw angle causes cyclic variation in the angle of attack. For this particular rotor the $-30^{\circ}$ yaw angle and $10 \%$ tower shadow cause a $12^{\circ}$ peak-to-peak change in angle of attack. The axial induction factor indicates the blade is moderately loaded at the $75 \%$ station. 
Table 6.1. Baseline Conditions for the Rigid-Hub Sensitivity Studies

Rotor

Pitch angles

Mass balance

Wind speed

Yaw angle

Vertical wind shear

Horizontal wind shear

Tower shadow

Vertical wind speed

Dynamic stall parameters

Yaw stiffness

Blade flap frequency

Low-speed shaft length

Shaft tilt
SERI Combined Experiment

Each blade at $+5^{\circ}$

Ideal

37. $\mathrm{ft} / \mathrm{sec}$

$-300^{\circ}$

0.14 power law profile

0.0

$10 \%$

0.0

$\mathrm{A}=0.0, \mathrm{~B}=0.7$

"fixed"

$4.06 \mathrm{p}$

$5 \mathrm{ft}$

$0^{\circ}$

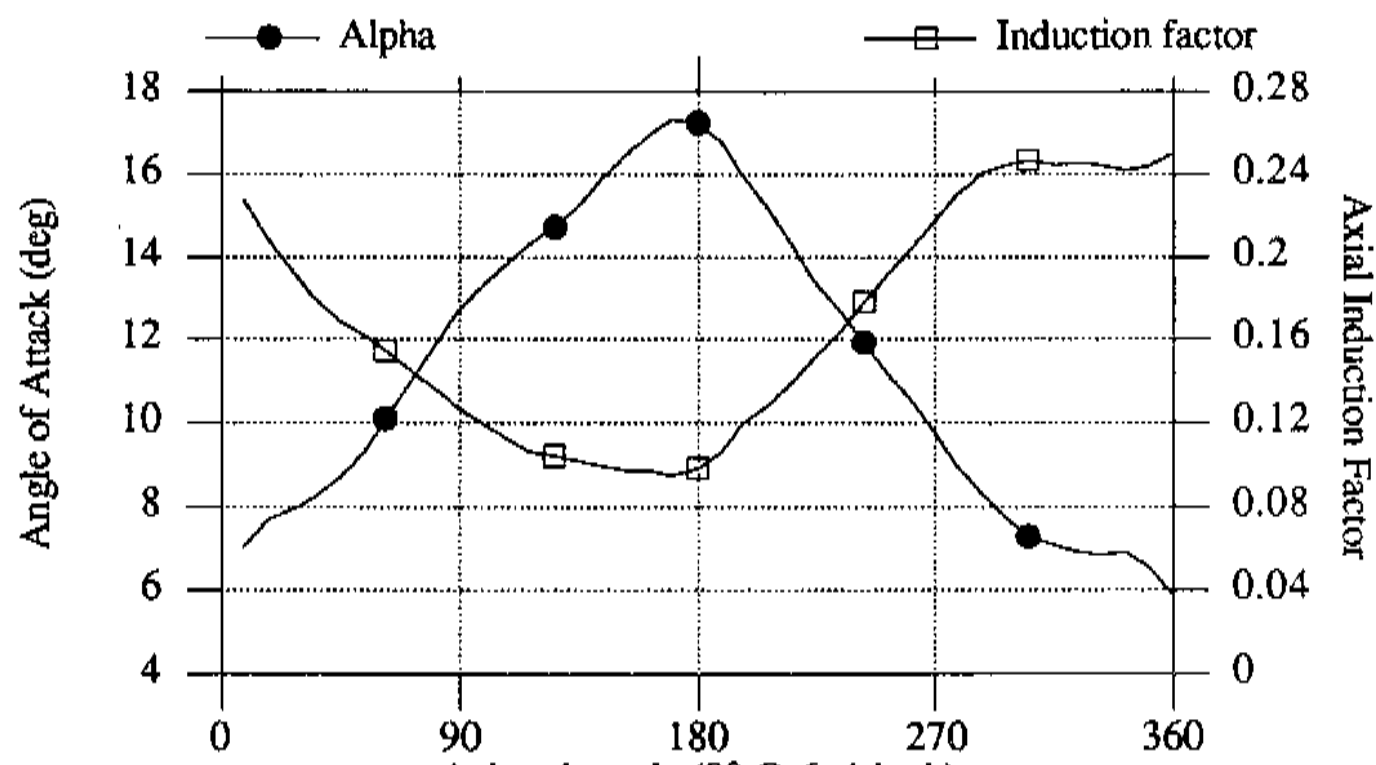

Figure 6.5 Variation of angle of attack and axial induction factor at the $75 \%$ station in the baseline configuration.

Figure 6.6 shows the cyclic nature of the root flap and yaw moments. The flap moment contains significant $1 p$ and $4 p$ cyclics caused primarily by the yaw angle and tower shadow. The blade flap natural frequency near $4 \mathrm{p}$ results in a large response at 4p. The 4p flap moment causes a significant 3p yaw moment while the mean yaw moment results from the $1 \mathrm{p}$ flap moment. Note there is virtually no $1 \mathrm{p}$ yaw moment as long as the rotor is mass and pitch balanced. 


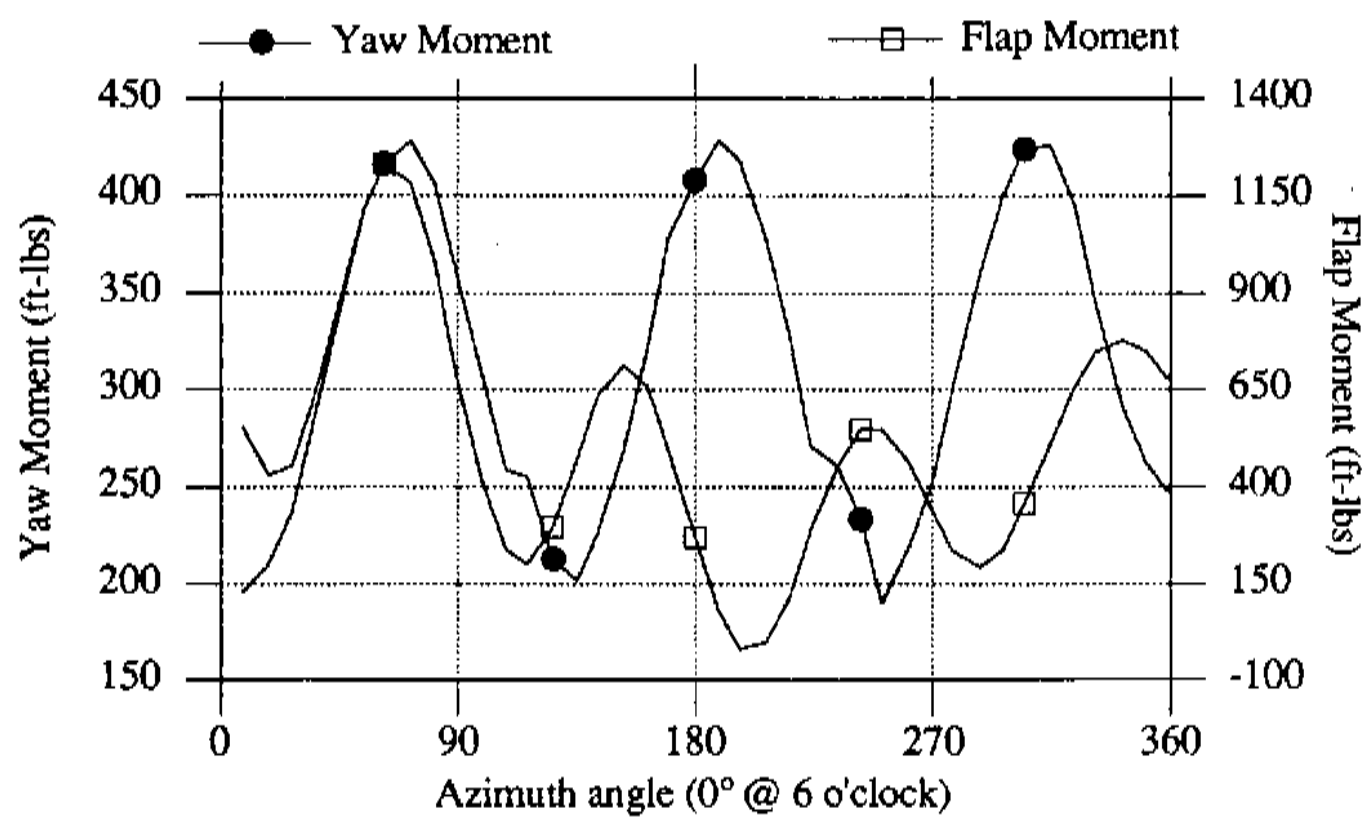

Figure 6.6 Yaw and root flap moments in the baseline configuration.

Figure 6.7 shows the stall hysteresis predicted by YawDyn when the rotor is in the baseline configuration. The upper hysteresis loop constant, $\mathrm{A}$, equals 0.0 , and the lower loop constant B equals 0.7 in this figure. Thus there is no dynamic overshoot of the $\mathrm{CL}$ but there is considerable hysteresis as the angle of attack is decreasing. The calculation is shown for azimuth angles between $8^{\circ}$ and $359^{\circ}$. The small loop observed in the lower left portion of the figure is caused by the tower shadow.

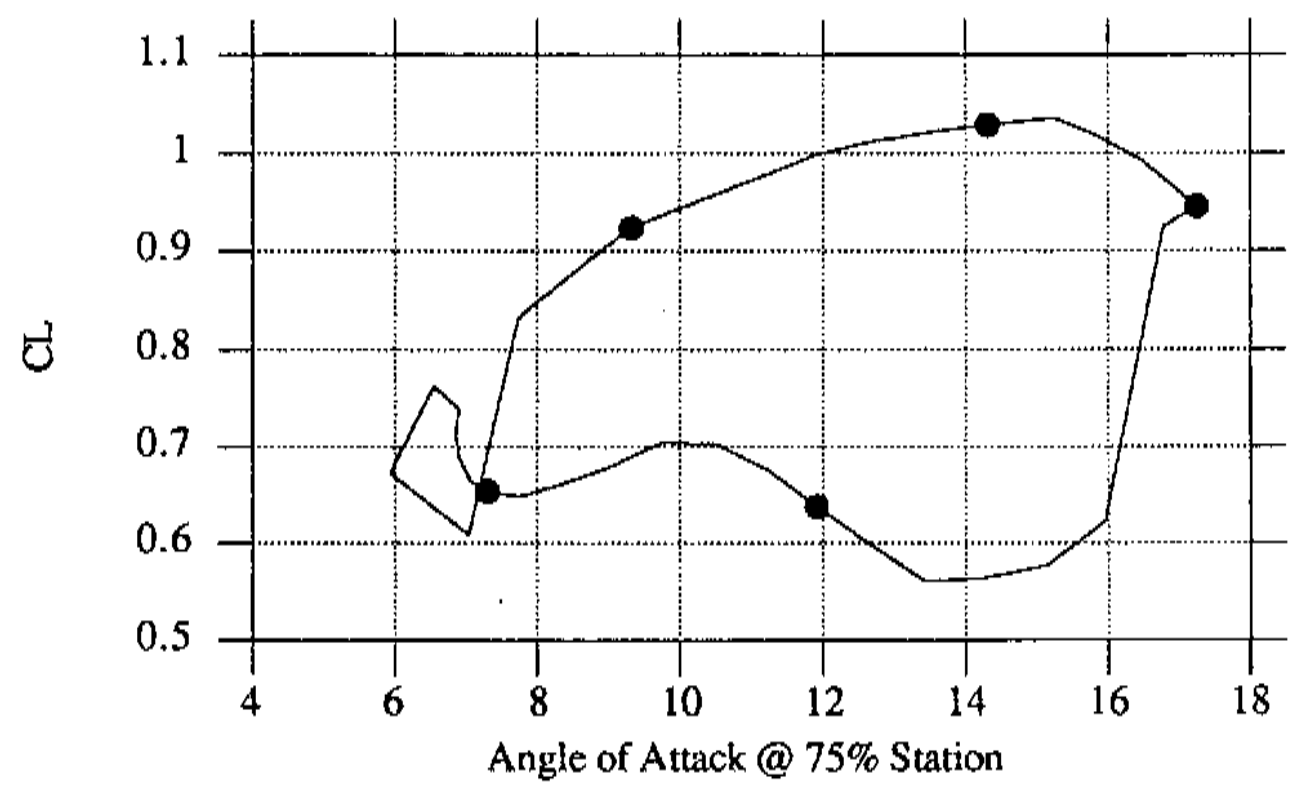

Figure 6.7 Stall hysteresis at the $75 \%$ station in the baseline configuration.

Having established the major characteristics of the baseline operation, it is useful to examine the effects of changes in some of the machine, wind and model parameters 
which can be selected or adjusted in YawDyn. Rather than present the detailed results for each condition that were presented above for the baseline condition, the key results are summarized in Tables 6.2 and 6.3 below. In all cases the basic waveforms and character of the rotor response remains the same, though there are very large variations in the loads and the relative magnitudes of the various load harmonics. (The only exceptions to this are the cases where the yaw stiffness option is employed in YawDyn. In those cases the waveforms are altered in both harmonic content and amplitude.)

In these tables the first two columns list the parameter under investigation and the new value of the parameter. In each line of the tables the parameter listed is the only change from the baseline configuration. The remaining columns tabulate the loads and the percent change in the load from the baseline result. A quick scan down any given column will show the parameters which have the greatest effect on the given load.

First examine the mean yaw load columns in Table 6.2. The largest changes in mean yaw moment are caused by horizontal wind shear, the neglect of dynamic stall or skewed wake corrections, vertical wind component, and yaw angle. A number of parameters have little influence on the mean yaw moment. Perhaps the most surprising is the "low speed shaft length" (the distance from the hub to the yaw axis). Prior to this research it was common to hear discussion of changes in this moment arm causing changes in a rotor's yaw behavior. But, as noted earlier, the dominant yaw loads from a rigid rotor are caused by differences in flap moments. These moments are essentially independent of the shaft length, hence the mean yaw moment is not greatly influenced by the shaft length.

It is important to note the importance of the dynamic stall and skewed wake corrections in the aerodynamic analysis. The mean yaw moment is over two orders of magnitude too small when classical blade-element/momentum aerodynamics methods are used to estimate the yaw loads.

Next examine the 3p yaw moments. Since these loads result primarily from the $4 p$ flap moments, any factor which influences the $4 \mathrm{p}$ flap loads can exert a strong influence on the $3 p$ yaw loads. The dynamic stall and skewed wake corrections and vertical wind component which were so important to mean yaw loads have a lesser influence on the cyclic loads. But tower shadow, blade or yaw axis natural frequency and horizontal wind shear have a striking influence on the cyclic yaw loads.

$1 \mathrm{p}$ yaw moments are not included in Table 6.2 because the only source of $1 \mathrm{p}$ yaw loads is an imbalance in the rotor. Mass or pitch imbalance can cause large 1p yaw loads as indicated in the footnotes to the table. Neither mass nor pitch imbalance cause significant flap loads or mean and 3p yaw loads unless the imbalance is sufficient to excite system natural frequencies by means of nacelle pitch or yaw motion.

Mean flap moments change less than the mean yaw moments for any condition. This emphasizes the fact that the yaw loads result from small differences in the large flap loads. Thus it is reasonable to expect that the yaw loads will be more sensitive to changes in wind or machine characteristics than the flap loads. Elimination of the flap degree of freedom changes the mean flap moment by not allowing the mean coning angle to change in response to airloads. The other parameter which has a strong influence on mean yaw loads is the yaw angle. This is expected as the yaw angle reduces the wind component normal to the rotor.

Cyclic flap moments vary more than the mean flap moments but less than the yaw loads. As noted above the blade and yaw column natural frequencies and the tower shadow and horizontal wind shear have the greatest influence on cyclic flap loads. 
Table 6.2 Sensitivity of yaw moments to changes in machine and wind parameters

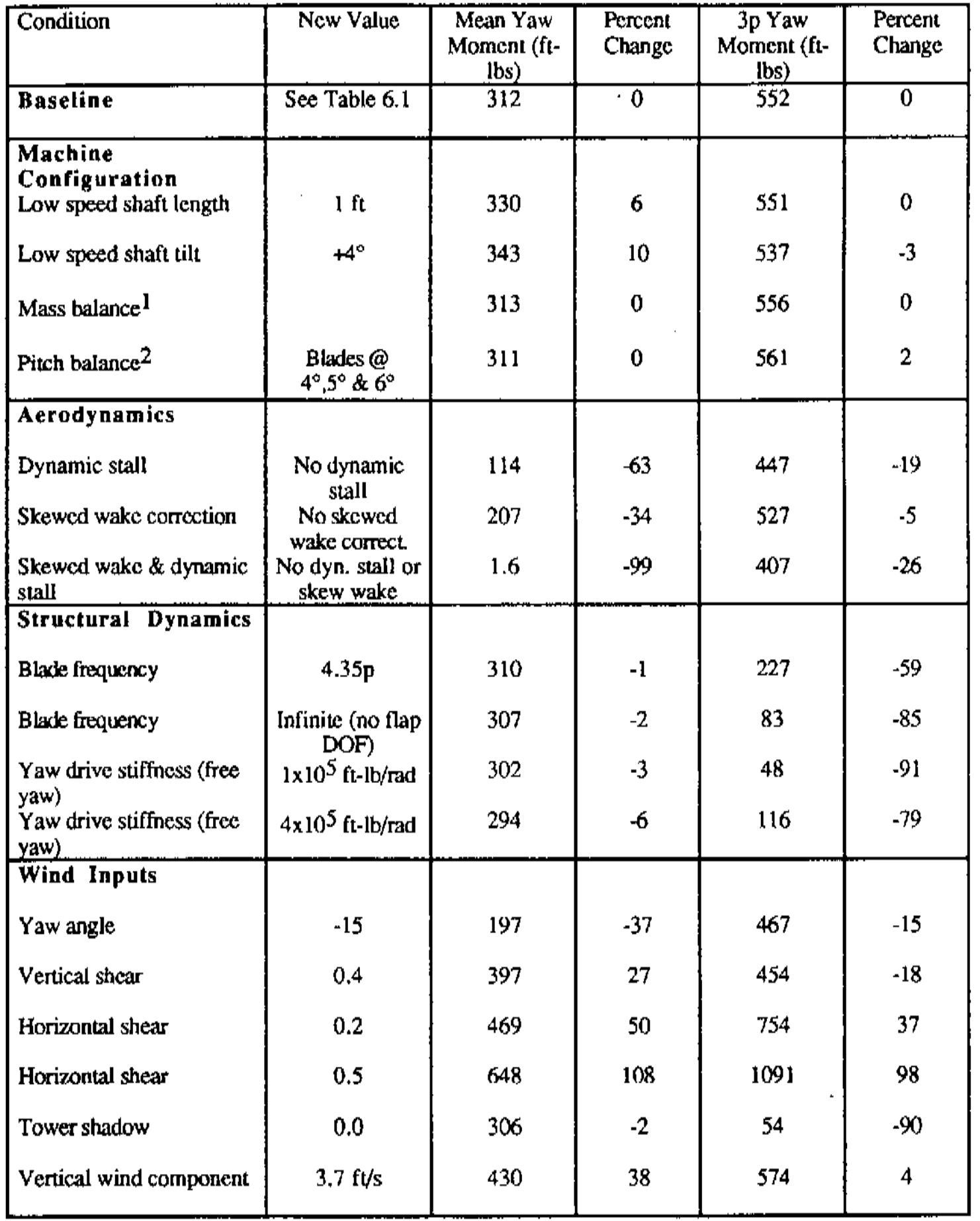

\section{Notes:}

1. Blade imbalance causes a $1 \mathrm{p}$ yaw moment of $190 \mathrm{ft}-\mathrm{lbs}$, calculated using cquation 3.11. The mass imbalance is assumed to have little effect on other harmonics. Mass imbalance is not modeled directly in YawDyn.

2. Pitch imbalance induces an $78 \mathrm{ft}-\mathrm{lb}, 1 \mathrm{p}$ yaw moment. In the baseline condition there is zero lp cyclic yaw moment. 
Table 6.3 Sensitivity of blade root flap moments to changes in machine and wind parameters.

\begin{tabular}{|c|c|c|c|c|c|c|c|}
\hline Condition & New Value & $\begin{array}{l}\text { Mean } \\
\text { Flap } \\
\text { Moment } \\
\text { (ft-lbs) }\end{array}$ & $\begin{array}{l}\text { Percent } \\
\text { Change }\end{array}$ & $\begin{array}{l}\text { lp Flap } \\
\text { Moment } \\
\text { (ft.llbs) }\end{array}$ & $\begin{array}{l}\text { Percent } \\
\text { Change }\end{array}$ & $\begin{array}{c}\text { 4p Flap } \\
\text { Moment } \\
\text { (ft.lbs }\end{array}$ & $\begin{array}{l}\text { Percent } \\
\text { Change }\end{array}$ \\
\hline Baseline & Sce Table 6.1 & 512 & 0 & 274 & 0 & 302 & 0 \\
\hline $\begin{array}{l}\text { Machine } \\
\text { Configuration } \\
\text { Low speed shaft } \\
\text { length }\end{array}$ & $1 \mathrm{ft}$ & 512 & 0 & 274 & 0 & $\begin{array}{l}302 \\
310\end{array}$ & 0 \\
\hline Low speed shatt tul & $+4^{\circ}$ & 458 & -11 & 262 & 4 & 310 & 3 \\
\hline Mass balance & & 512 & 0 & 274 & 0 & 302 & 0 \\
\hline Pitch balance & $\begin{array}{l}\text { Blades@ } \\
4^{\circ}, 5^{\circ} \& 6^{\circ} \\
\end{array}$ & 549 & 7 & 297 & 8 & 355 & 18 \\
\hline $\begin{array}{l}\text { Aerodynamics } \\
\text { Dynamic stall }\end{array}$ & $\begin{array}{l}\text { No dynamic } \\
\text { stall }\end{array}$ & 584 & 14 & 240 & -12 & 283 & -6 \\
\hline $\begin{array}{l}\text { Skewed wake } \\
\text { correction }\end{array}$ & $\begin{array}{l}\text { No skewed } \\
\text { wake correct. }\end{array}$ & 511 & 0 & 228 & -17 & 329 & 9 \\
\hline $\begin{array}{l}\text { Skewed wake \& } \\
\text { dynamic stall }\end{array}$ & $\begin{array}{l}\text { No dyn. stall } \\
\text { or skewed } \\
\text { wake correct. }\end{array}$ & 587 & 15 & 227 & -17 & 295 & -2 \\
\hline $\begin{array}{l}\text { Structural } \\
\text { Dy namics } \\
\text { Blade frequency }\end{array}$ & $4.35 p$ & 516 & 1 & 273 & 0 & 147 & -.51 \\
\hline Blade frequency & $\begin{array}{l}\text { Infinite (no } \\
\text { flap DOF) }\end{array}$ & 645 & 26 & 275 & 0 & 21 & -93 \\
\hline $\begin{array}{l}\text { Yaw drive stiffness } \\
\text { (free yaw) }\end{array}$ & $\begin{array}{l}1 \times 10^{5} \mathrm{ft}- \\
\mathrm{lb} / \mathrm{rad}\end{array}$ & 505 & -1 & 261 & -5 & 63 & -79 \\
\hline $\begin{array}{l}\text { Yaw drive stiffness } \\
\text { (free yaw) }\end{array}$ & $\begin{array}{c}4 \times 10^{5} \mathrm{ft}- \\
\mathrm{lb} / \mathrm{rad}\end{array}$ & 513 & 0 & 271 & -1 & 49 & -84 \\
\hline $\begin{array}{l}\text { Wind Inputs } \\
\text { Vertical shear }\end{array}$ & 0.4 & 449 & -12 & 268 & -2 & 238 & $-2 I$ \\
\hline Yaw angle & -15 & 632 & 23 & 151 & -45 & 288 & -5 \\
\hline Horizontal shear & 0.2 & 498 & -3 & 398 & 45 & 415 & 37 \\
\hline Horizontal shear & 0.5 & 457 & -11 & 563 & 105 & $\$ 62$ & 86 \\
\hline Tower shadow & 0.0 & 526 & 3 & 297 & 8 & 110 & -64 \\
\hline $\begin{array}{l}\text { Vertical wind } \\
\text { component }\end{array}$ & $3.7 \mathrm{ft} / \mathrm{s}$ & 511 & 0 & 297 & 8 & 328 & 9 \\
\hline
\end{tabular}

Engineers designing a new rotor or troubleshooting an existing system may find it helpful to conduct sensitivity studies such as these for their system. Only a few hours are required to run enough cases to identify the most critical parameters. This information can then be used to guide selection of more detailed testing or analysis 
conditions. Knowledge of the most critical parameters permits focus of resources on the most fruitful (or harmful) areas.

The two cases run to investigate the sensitivity to yaw stiffness deserve additional discussion. These cases were nun with the free-yaw option selected (the only time the yaw stiffness is used) and are intended to simulate the stiffness of a yaw drive. They allow interaction of the flap and yaw degrees of freedom. Figure 6.8 shows the timehistories of the yaw and flap moments for the case when the yaw drive equivalent stiffness is $4 \times 10^{5} \mathrm{ft}-1 \mathrm{~b} / \mathrm{rad}$. Notice the motions have not yet reached a steady-state condition after four revolutions of the rotor. This is because the trim solution applies only to the flap degree of freedom with the rotor fixed in yaw. The transient solution marks the beginning of the "trimming" process for the combined yaw and flap degreesof-freedom. The load harmonics given in the tables above are for the fourth revolution of the rotor when the yaw stiffness option is analyzed.

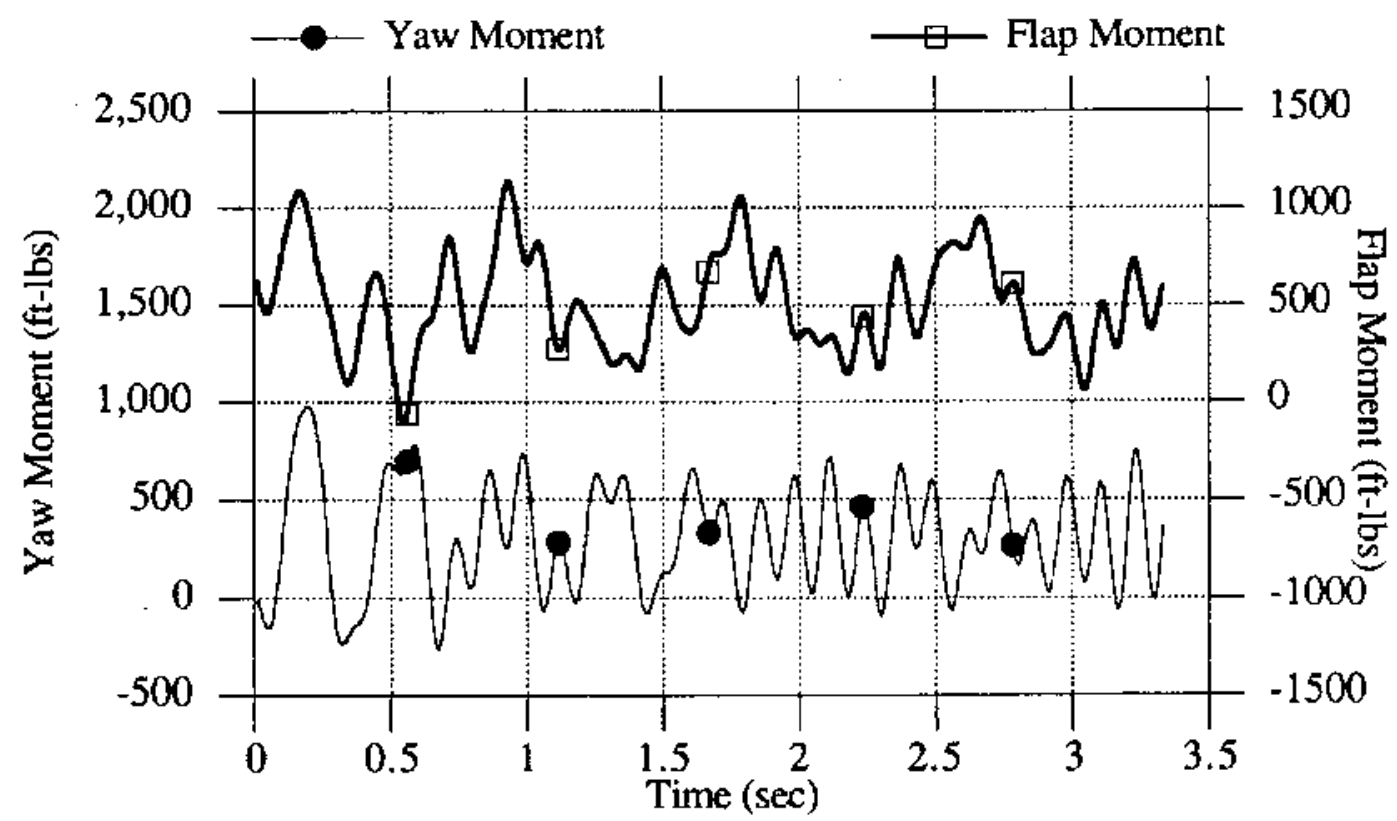

Figure 6.8. Yaw and flap moments with the rotor yawing against an effective stiffness of $4 \times 10^{5} \mathrm{ft}-\mathrm{lb} / \mathrm{rad}$. All other parameters match the baseline conditions of Table 6.1.

It is clear that selection of the yaw drive stiffness can greatly influence the yaw and flap loads. Obviously, if the stiffness is very high then loads will be the "fixed-yaw" values. If the stiffness places the yaw and flap motions in resonance then considerable amplification will result. A low stiffness can be used to reduce rotor loads if the selection is made with great care.

\subsection{Teetering rotor configuration}

The ESI-80 rotor was selected for the sensitivity studies with a teetering hub. This rotor was selected because it is one of the few teetering systems commercialized in the U.S. and it is well-known to the principal investigator (who participated in the design of the system). It is also the baseline configuration for the Advanced Wind Turbine design being conducted by R. Lynette and Associates under contract to SERI. The rotor is described in detail in Appendix B. 
Baseline conditions for the study are summarized in Table 6.4 below. Table 6.5 summarizes the results of the sensitivity study for the yaw moment. In each case the conditions were as noted in Table 6.4, except for the single condition varied as noted in the "Condition" and "New Value" columns in Table 6.5.

Table 6.4. Baseline Conditions for the Teetered-Hub Sensitivity Studies

$\begin{array}{ll}\text { Rotor } & \text { ESI-80 } \\ \text { Pitch angles } & \text { Both blades at } 0^{\circ} \\ \text { Mass balance } & \text { Ideal } \\ \text { Wind speed } & 33.5 \mathrm{ft} / \mathrm{sec} \\ \text { Yaw angle } & 200^{\circ} \\ \text { Vertical wind shear } & 0.15 \text { power law profile } \\ \text { Horizontal wind shear } & 0.0 \\ \text { Tower shadow } & 10 \% \\ \text { Vertical wind speed } & 0.0 \\ \text { Dynamic stall parameters } & \mathrm{A}=0.0, \mathrm{~B}=0.5 \\ \text { Yaw stiffness } & \text { "fixed" } \\ \text { Low-speed shaft length } & 6.79 \mathrm{ft} \\ \text { Shaft tilt } & 0^{\circ}\end{array}$

Characteristics of the rotor behavior are shown for the baseline configuration in Figures 6.8 and 6.9. The rotor teeters at the expected $1 \mathrm{p}$ frequency and the angle of attack history is also dominated by $1 p$ fluctuations. However, the angle of attack also contains $2 p$ components due to variations in local wind (including induced velocity) and a pulse at $0^{\circ}$ azimuth due to tower shadow. The yaw and flap moments exhibit $2 \mathrm{p}$ oscillations with small pulses due to tower shadow. The flap moment also contains some energy at $1 \mathrm{p}$.

Several observations are noteworthy in Table 6.5. First, notice the mean yaw moment is nearly tripled and the $2 \mathrm{p}$ moments increase nearly eightfold when the same rotor is not allowed to teeter. This illustrates the relative importance of the flap moments (which are not transmitted to the nacelle of a teetering rotor) and the horizontal force in determining the yaw moments. However, if the teeter stops are contacted (such as in the case with a $40^{\circ}$ yaw angle) then the advantage of the teetering rotor is lost and the mean and cyclic yaw moments increase dramatically.

The teetering rotor sensitivity to wind inputs is markedly different from the rigid rotor. Note the increased importance of vertical wind shear and diminished importance of horizontal shear and vertical component of wind. Dynamic stall had little effect on the mean yaw moment in the case examined here. This is quite a contrast to the importance of dynamic stall on a rigid rotor.

The distance from the hub to the yaw axis was of minor significance for rigid rotor yaw loads because of the dominance of the blade flap moments. A teetering rotor depends upon the horizontal rotor force to generate yaw moments. Thus it is not surprising that the yaw load is sensitive to the shaft length. Likewise, the yaw moment is more sensitive to shaft tilt for a teetering rotor than a rigid rotor. 


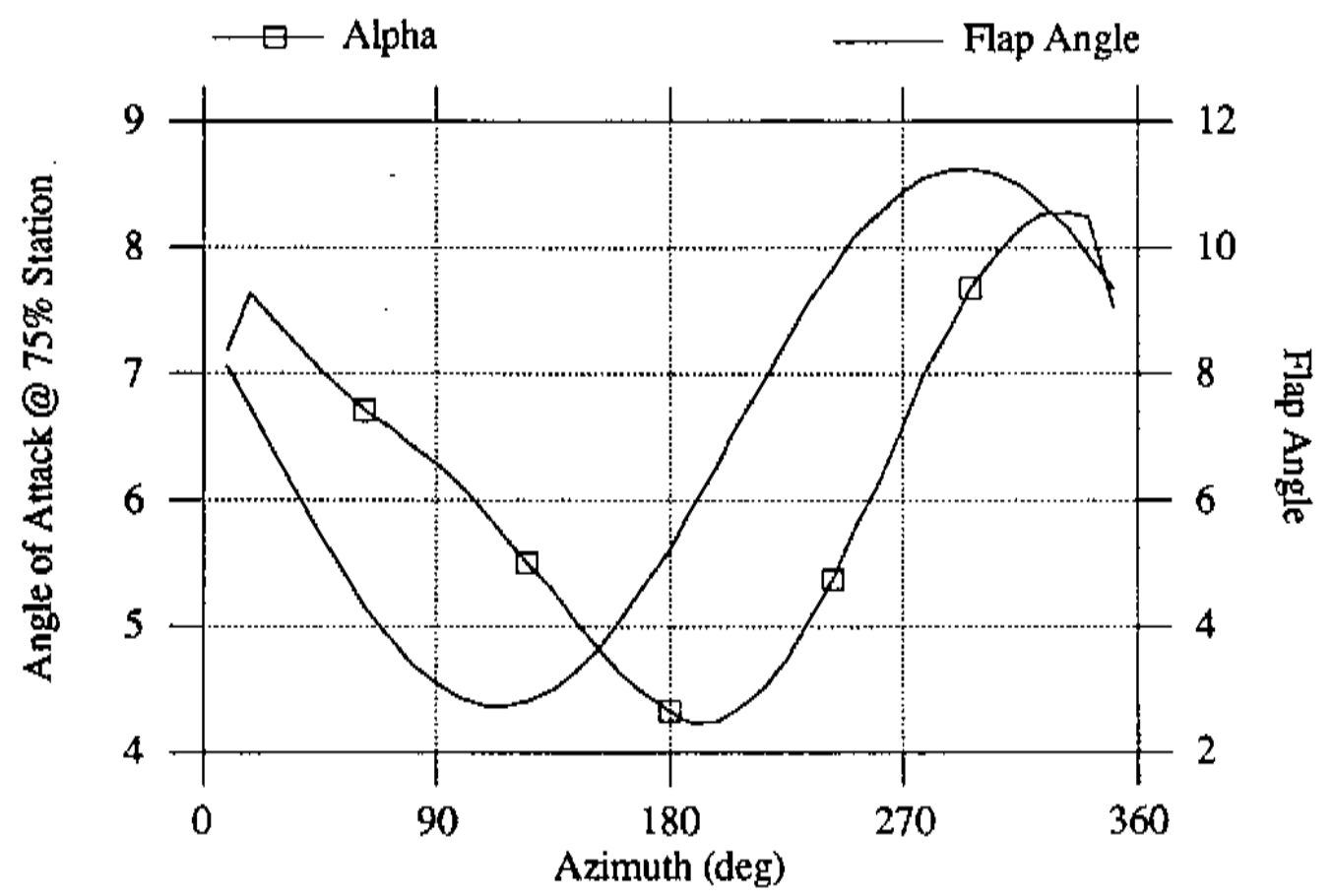

Figure 6.8 Flap angle and angle of attack history for the baseline teetering rotor case. The teeter angle is the difference between the flap angle and the precone angle of $7^{\circ}$.

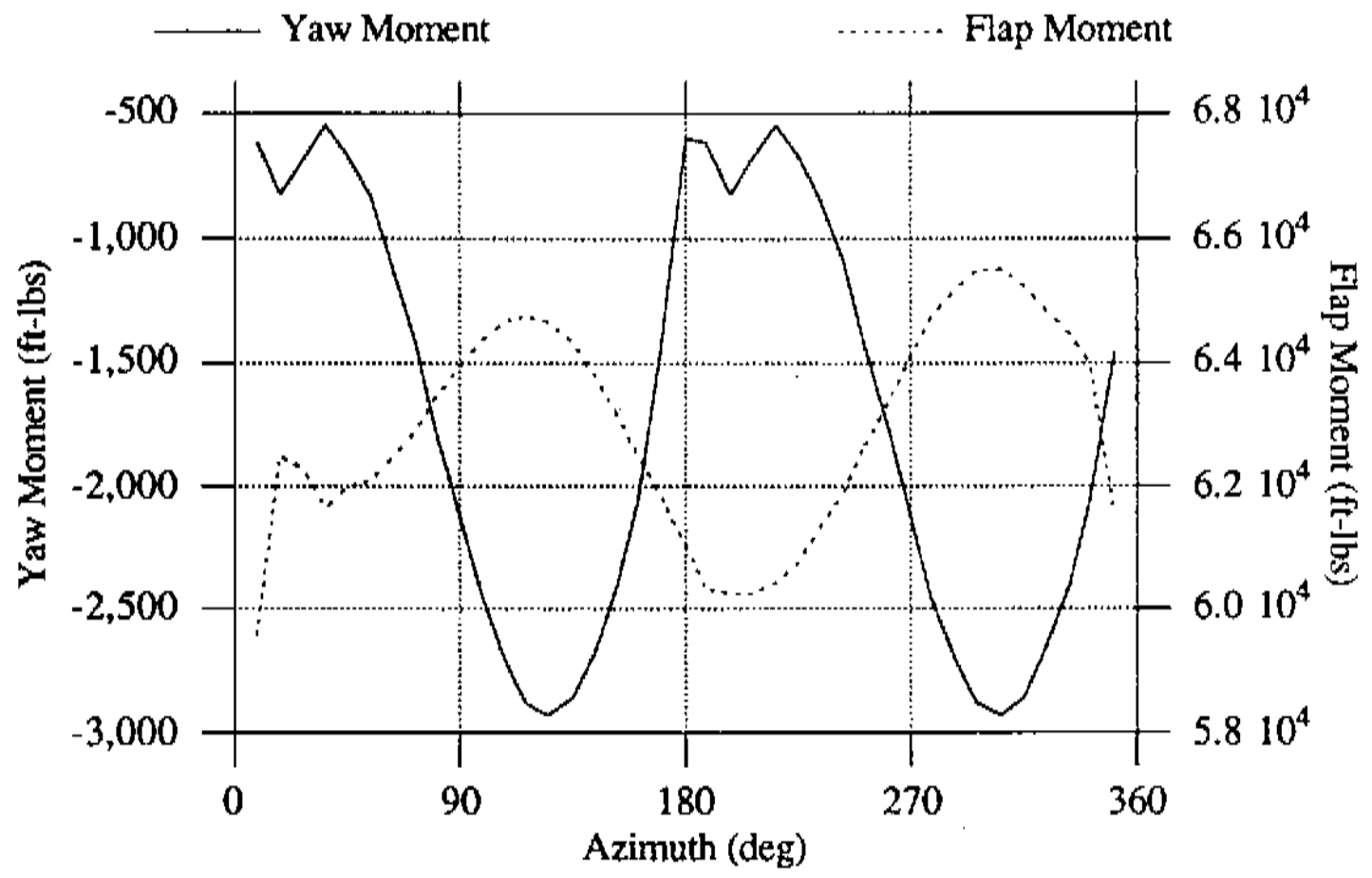

Figure 6.9 Yaw and root aerodynamic flap moment for the baseline teetering rotor case. 
Table 6.5 Results of the teetered-hub sensitivity studies for the yaw moment. ESI-80 turbine operated at the conditions of Table 6.4, except as noted.

\begin{tabular}{|l|c|c|c|c|c|}
\hline \multicolumn{1}{|c|}{ Condition } & New Value & $\begin{array}{c}\text { Mean Yaw } \\
\text { Moment (ft-lbs) }\end{array}$ & $\%$ Change & $\begin{array}{c}\text { 2p Yaw } \\
\text { Moment (ft-lbs) }\end{array}$ & \% Change \\
\hline Baseline & - & -1685 & $0 \%$ & 1219 & $0 \%$ \\
\hline Machine & & & & & \\
Configur ation & & & & & \\
Shaft Length & $1.79 \mathrm{ft}$ & -591 & $-65 \%$ & 632 & $48 \%$ \\
Shaft Tilt & $+4^{\circ}$ & -2786 & $65 \%$ & 1623 & $33 \%$ \\
Shaft Tilt & $-4^{\circ}$ & -581 & $-66 \%$ & 947 & $-22 \%$ \\
Teeter DOF & None & -4634 & $175 \%$ & 9568 & $685 \%$ \\
\hline & & & & & \\
Aerodynamics & & & & & \\
Skewed Wake & None & -1459 & $-13 \%$ & & $-22 \%$ \\
Correction & & & & & \\
Dynamic Stall & None & -1683 & $0 \%$ & 1146 & $-6 \%$ \\
\hline Wind Inputs & & & & & \\
Vertical Shear & 0.4 & -2499 & $48 \%$ & 2750 & $126 \%$ \\
Horizontal Shear & 0.5 & -706 & $-58 \%$ & 1149 & $-6 \%$ \\
Horizontal Shear & 0.2 & -1279 & $-24 \%$ & 844 & $-31 \%$ \\
Tower Shadow & 0.0 & -1646 & $-2 \%$ & 1139 & $-7 \%$ \\
Vertical Wind & $3.7 \mathrm{f} / \mathrm{sec}$ & -1770 & $5 \%$ & 1402 & $15 \%$ \\
Yaw angle & $+40^{\circ *}$ & -11620 & $590 \%$ & 19180 & $1473 \%$ \\
Yaw angle & $+30^{\circ}$ & -2022 & $20 \%$ & 1664 & $37 \%$ \\
Yaw angle & $0^{\circ}$ & -511 & $-70 \%$ & 608 & $-50 \%$ \\
Yaw angle & $-200^{\circ}$ & 614 & $-136 \%$ & 886 & $-27 \%$ \\
Yaw angle & $-30^{\circ}$ & 887 & $-153 \%$ & 1218 & $0 \%$ \\
Yaw angle & $-40^{\circ}$ & 4505 & $-367 \%$ & 7160 & $487 \%$ \\
\hline
\end{tabular}

* Significant teeter stop impacts in this condition

\subsection{A Comparison of the Free-Yaw Behavior of Rigid and Teetering Rotors}

One final subject is not a sensitivity study per se, but it examines the influence of rotor configuration upon the free-yaw behavior of a turbine. To accomplish this two rotors were analyzed. One is the ESI-80 teetering rotor analyzed earlier. For purposes of comparison a second, imaginary, rotor with a rigid bub was created in YawDyn. This rotor is called the Rigid-80. But all other features of the rotor were selected to match the ESI-80 insofar as possible. The Rigid-80 has three blades, but the radius, coning, solidity, pitch angle, airfoil and planform are identical to the ESI-80. Thus the aerodynamic loading and power output of the rotors are the same in a steady, uniform. wind. Different blade stiffnesses were analyzed for the Rigid- 80 to determine the influence of flap natural frequency on the free-yaw behavior. The blade and yaw mass moments of inertia were identical for both rotors.

Free-yaw performance was examined by simulating the release of a yaw brake on the rotor when it is initially at a $\pm 20^{\circ}$ yaw angle. The yaw motion was then calculated until the rotor reaches an equilibrium location in the steady wind conditions. Varying wind shears were employed to determine their effect on the final equilibrium yaw angle. Figure 6.10 shows a typical set of results for both rotors. Notice that the rotors move to the same equilibrium yaw angle regardless of whether they were released from $+20^{\circ}$ or $-20^{\circ}$. This is expected since the initial angle should affect only the yaw rates and the time to reach equilibrium. The example shown has a rather severe, steady, horizontal 
wind shear coefficient of 0.2 as well as vertical shear and tower shadow. Thus the rotors will not align with the wind direction (yaw angle of $0^{\circ}$ ). It is interesting to note that the rigid rotor shows greater misalignment in this case than its teetering counterpart. Notice also that the Rigid- 80 demonstrates a slight "overshoot" in its response while the ESI-80 does not. The rigid rotor also reaches its final equilibrium position much more quickly (even in the case where it yawed over $40^{\circ}$ ). This is typical of most of the cases examined and indicates the higher yaw moments which are observed on rigid rotors.

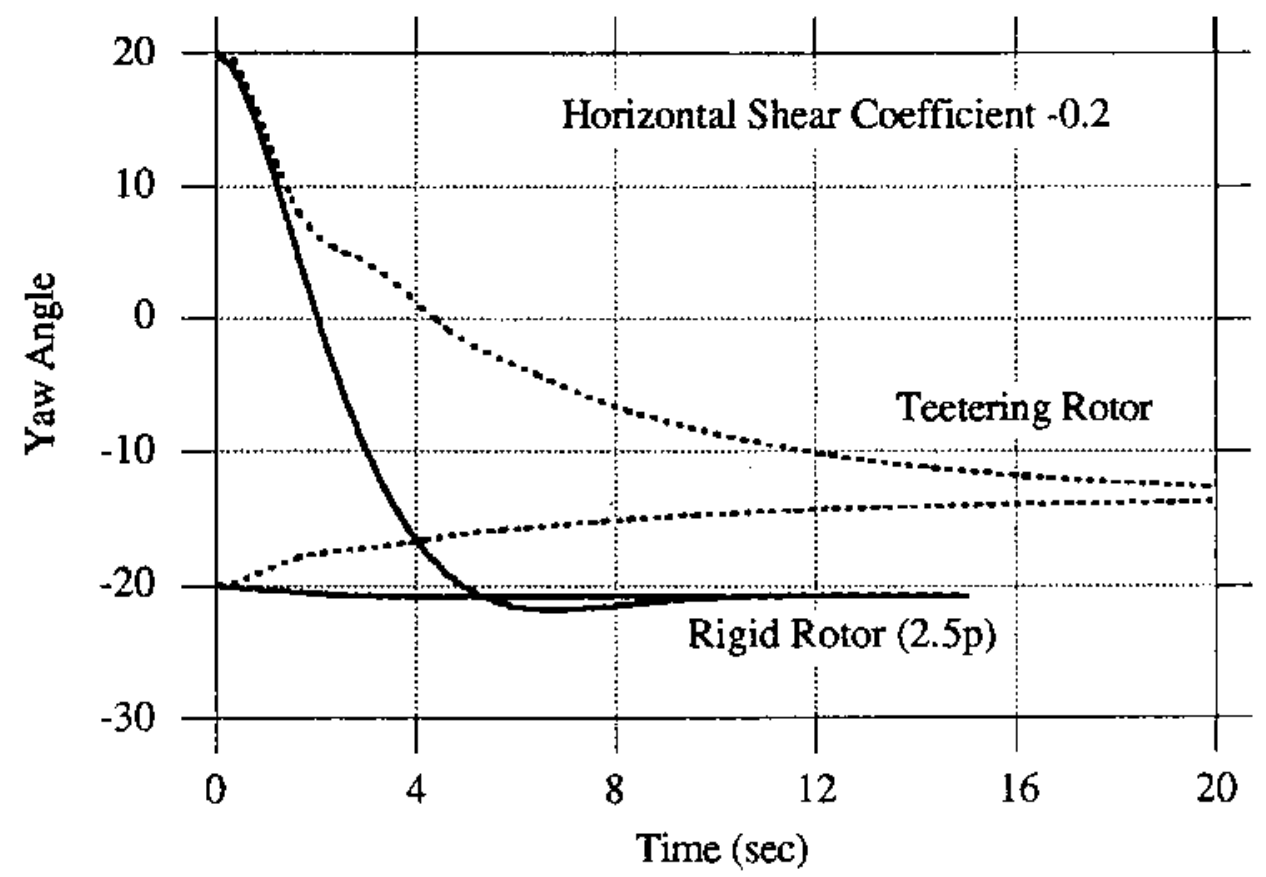

Figure 6.10 Free-yaw time history of the two rotors after release from rest at a $\pm 20^{\circ}$ yaw angle. Wind speed, $33.5 \mathrm{ft} / \mathrm{s}$; Vertical wind shear, 0.14 power law; Tower shadow, $10 \%$; Rigid- 80 blade stiffness, $2.5 p$ (rotating).

Figure 6.11 shows the influence of blade stiffness and hub articulation on the yaw response when there is no horizontal wind shear. It is clear that the blade stiffness affects the yaw rates and equilibrium position. In a case such as this where only vertical non-uniformities are acting on the rotor, the "softer" rotors show the larger yaw error. When horizontal shears are present the stiff rotors show larger yaw misalignment. This behavior results from the greater phase shift between excitation and response for the softer rotors.

Figure 6.12 shows the results of similar calculations for various blade stiffnesses and horizontal shear coefficients. Notice that the teetering rotor shows the least overall sensitivity to wind shear and the $2.5 \mathrm{p}$ rotor shows the greatest variation in equilibrium yaw angle for the same range of horizontal shears. The results for blades with higher natural frequencies ( $3.5 p$ and $4.5 p$ ) are not shown, but were found to be very similar to those for the $2.5 p$ rotor. 


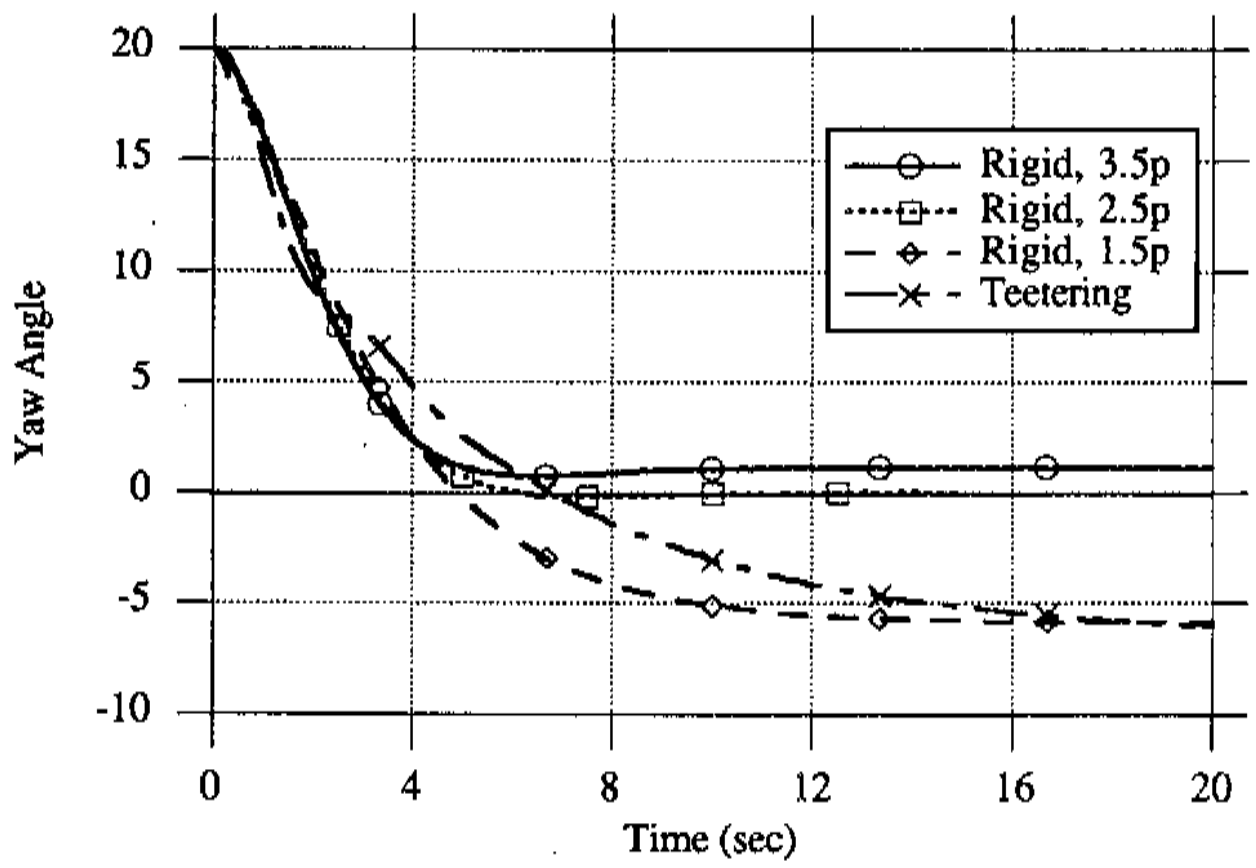

Figure 6.11 Free-yaw time history of four rotors after release from rest at a $20^{\circ}$ yaw angle. Wind speed, $33.5 \mathrm{ft} / \mathrm{s}$; Vertical wind shear, 0.14 power law; Tower shadow, $10 \%$.

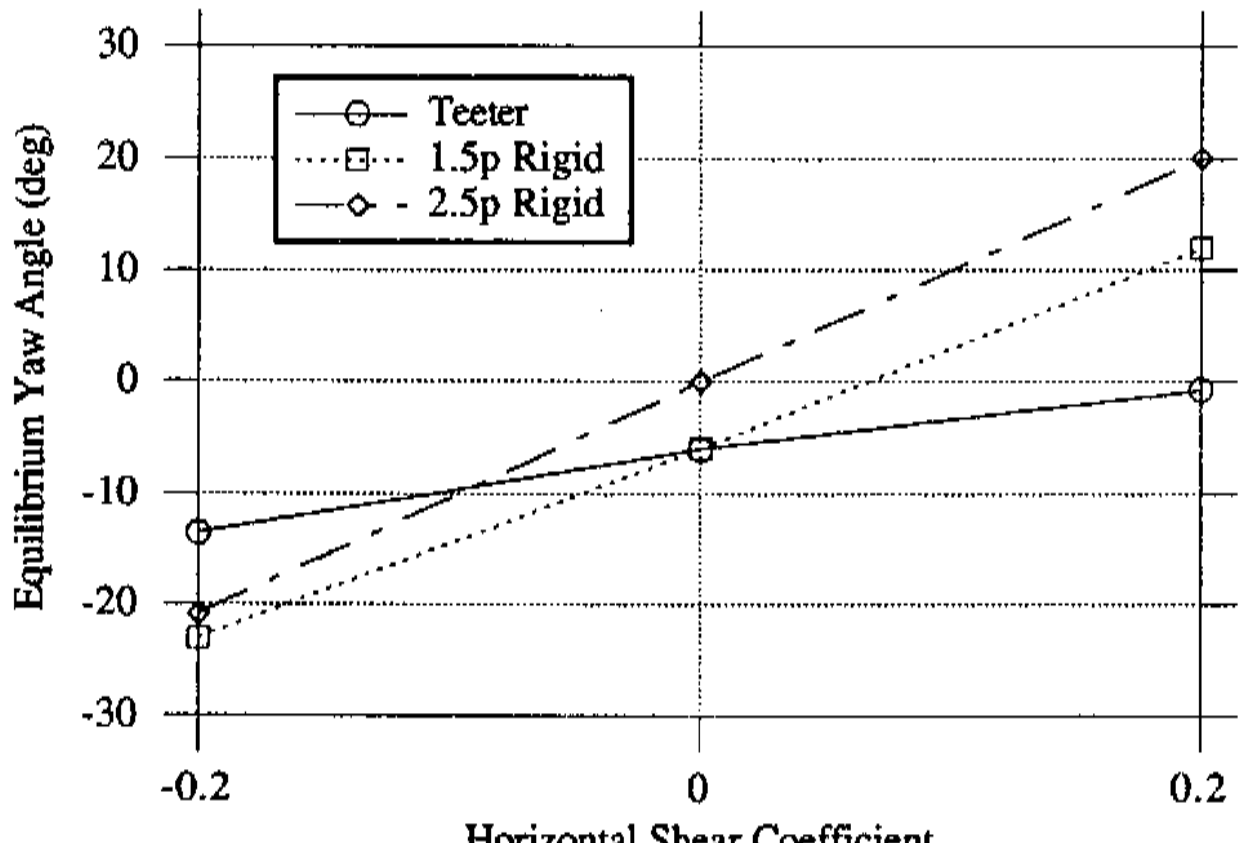

Figure 6.12 Effect of horizontal wind shear on the equilibrium yaw angle. Wind speed, $33.5 \mathrm{ft} / \mathrm{s}$; Vertical wind shear, 0.14 power law; Tower shadow, $10 \%$. 


\subsection{Conclusions and Recommendations}

\subsection{The YawDyn Model}

The YawDyn model has been developed as a basic design tool and as an aid to understanding the features of a wind turbine which are most important in influencing the system's yaw behavior. The model is applicable to fixed or free yaw motions of rigid-rotor, two- or three-blade turbines and teetering hub turbines. YawDyn calculates yaw loads and motions of a turbine subjected to time-varying wind speeds, wind directions and wind shears. Calculation of blade flap loads and motions is also accomplished by the program. The methods selected to represent each element of the turbine were as simple as possible while retaining the essential physical features.

This research has demonstrated the importance of stall hysteresis and skewed wake corrections in the aerodynamics analysis. Without these two modifications to quasisteady blade-element/momentum analysis the predicted yaw loads are much lower than those observed on a rigid rotor. In the present model the skewed wake corrections are a quasi-steady implementation of the Pitt and Peters dynamic inflow theory. The importance of the inertial lag of dynamic inflow was not explored.

The model has been tested by comparisons with the FLAP program and with test data from the SERI Combined Experiment rotor and with 1/20-scale wind tunnel tests of a rigid-hub model of the Mod-2 rotor. The comparisons are generally favorable. YawDyn consistently predicts the correct qualitative trends, even when those trends are not obvious. The quantitative accuracy is less consistent. In many cases the mean and cyclic yaw and flap moments are estimated within $20 \%$. But in other cases the loads show larger errors. It is not known how much of the observed error is due to shortcomings in the model and how much is due to incomplete data for the actual input wind and uncertainties in the mass, stiffness and friction values for the machine.

The inadequacy of input wind data and machine characteristics is an inherent problem in all design codes, particularly during the preliminary design phase. The author has concluded, after validation studies on the Combined Experiment rotor, that the YawDyn program is sufficiently accurate for design purposes. Though the calculated loads will not be completely accurate, the errors will generally be less than uncertainties in selection of the design wind inputs. For example, a vertical wind component which is $10 \%$ of the mean wind can increase the yaw loads $40 \%$ (Table 6.2). The uncertainty in selection of a design vertical wind certainly exceeds $10 \%$. This is a rather bold statement and it certainly cannot be applied to all turbine configurations. But it does hint that attention must be paid to selection of extreme wind conditions and to design of rotors which are inherently insensitive to turbulence and other variations in the wind.

The analysis neglects tower motions, drive-train dynamics and all modes of blade vibration except the first flap mode. Thus, there will be many turbines which are not amenable to thorough analysis by YawDyn. Those turbines with complex system modes of vibration near a resonance with strong excitations cannot be expected to be accurately represented in YawDyn. However, even in this situation the method can still be of some assistance in the role for which it was intended: exploration of the important influences on the yaw loads or free-yaw motion. 


\subsection{Yaw Loads on_a_Rigid_Rotor}

1) Yaw loads result almost entirely from a summation of blade root flap moments on the Combined Experiment rigid rotor. The net lateral, horizontal aerodynamic force on the rotor is negligible by comparison. It is worth noting the yaw moment results from small differences in large (by comparison) flap moments. This makes results sensitive to minor changes in the flap moment distribution. Since the yaw moment is nearly independent of the $\mathrm{H}$-force, it is nearly independent of the distance from the hub to the yaw axis. Thus changing the low speed shaft length cannot be expected to greatly influence the yaw loads of a rigid rotor. One exception to this is the case when the rotor is not mass balanced. Then the cyclic lp yaw moment will be directly proportional to the yaw axis-hub distance. The yaw inertia may be significantly altered by changes in the distance from the rotor to the yaw axis. Thus increasing this distance may reduce yaw rates of a free-yaw machine.

2) If a rotor is dynamically mass balanced and aerodynamically balanced, then the yaw moments on a three-blade system will be composed of a mean moment, three-perrevolution (3p) cyclic moments, and integer multiples of $3 p$ cyclic moments. The mean yaw moment results from the $1 \mathrm{p}$ sine cyclic flap moment. The $3 \mathrm{p}$ yaw moments result from the $2 p$ and $4 p$ flap moments. If yaw moments with a large component of $1 p$ cyclic oscillations are observed on a rotor there is strong indication of a mass or blade pitch imbalance that can be eliminated by balancing of the rotor. On one test rotor the cyclic loads due to imbalance were the largest cyclic loads from any cause. Monitoring of yaw moments or accelerations is a sensitive indicator of rotor balance.

3) Corrections for skewed wake effects must be made to the basic blade element/momentum aerodynamics method when the rotor operates at a yaw angle. A quasi-steady version of Pitt and Peters dynamic inflow model gives improved agreement between predicted and measured yaw moments.

4) Dynamic stall hysteresis is important when determining the mean yaw moments on the SERI Combined Experiment rotor. More research is needed to determine the most appropriate dynamic stall model for wind turbine blades. The Gormont model used in this work is able to predict the correct hysteresis loop when two empirical constants are known a priori.

5) Horizontal wind shear is much more damaging to yaw drive systems of rigid rotors than vertical wind shear. This can be of great importance in highly turbulent sites or in wind farm arrays. Yaw loads on a well-balanced rotor will be more influenced by horizontal shear than by any other system input with the possible exception of a vertical component of wind speed. "Soft" yaw drives or soft rotors have been suggested as possible means to mitigate the effects of wind shear.

6) Vertical wind components produce much higher loads than comparable horizontal cross-flow components. This has important implications in site selection for very rigid rotors. Though vertical wind components will always be present in atmospheric turbulence, the designer must account for a probability of persistent and strong vertical winds at some sites.

\subsection{Yaw Loads on a Teetering_Botor}

1) The teetering rotor, by eliminating the load path from the blade flap moments to the rotor shaft, has lower yaw loads. The horizontal forces, which were largely negligible for a rigid rotor, become the dominant source of yaw loads for the teetering rotor. The 
net horizontal force is the resultant small difference of relatively large in-plane forces acting on the blades.

2) The yaw moments on a teetering rotor are strongly influenced by the distance from the yaw axis to the rotor, preconing, shaft tilt, and vertical and horizontal wind shears. The teetering rotor yaw moments are more sensitive to yaw error than the rigid rotor, though the magnimdes of the yaw loads remain lower.

\subsection{Yaw_Motions of Rigid and Teetering_Rotors}

1) Rigid rotors in free yaw demonstrate many of the characteristics of a damped oscillator. Some rotors are lightly damped and exhibit "overshoot" in their response to step changes in wind direction. Others exhibit heavily damped motion with no overshoot or ringing in the response. The blade flap stiffness and mass moment of inertia appear to be the most important factors in determining the nature of the yaw response.

2) A free-yaw, rigid rotor will experience blade flap moments due to Coriolis or gyroscopic effects. These 1 p cyclic moments are proportional to the yaw rate and can easily exceed the aerodynamic moments. It is imperative that the design focus on avoiding high yaw rates. Flap flexibility, nacelle mass (to increase the moment of inertia) and mechanical yaw dampers can be employed to reduce yaw rates.

3) Teetering rotors display more benign response to yaw disturbances than their rigidhub counterparts. This is due to the lower yaw moments of the teetering configuration. In all cases modeled the teetering rotor exhibited overdamped response to step changes in wind direction.

4) If a rotor is observed operating consistently misaligned with the wind direction, it is likely responding to persistent wind shear or vertical wind. Free-yaw rotors can be expected to misalign with the wind in complex terrain or wind farm arrays. The effect of misalignment on power production will typically be small. Misalignment may reduce loads on the rotor if it is allowed to yaw freely. This is because the equilibrium yaw angle will be determined by seeking the "balance point" in the blade flap loads. For example, if there is vertical shear the rotor will operate at the yaw angle that tends to minimize the lp variations in angle of attack. Yawing will induce advancing and retreating blade effects which can partially cancel the effects of the wind shear.

5) Following the point of item 4 above, if a yaw-controlled rotor is constrained to align with the wind direction it may experience higher cyclic loads than a free yaw system which is otherwise equal. Of course, if the free-yaw system experiences high yaw rates (because it is too responsive) then any possible advantage will quickly be negated.

\subsection{Recommendations for Additional Research}

This development is just one step in a long process of fully understanding yaw dynamics and learning to design rotors which take advantage of yaw behavior instead of being punished by it. Many extensions of this modelling technique are possible and needed. The most obvious are addition of other degrees of freedom and improvement of the aerodynamics calculations. It has become clear that the yaw behavior is a result of small differences in the loads on each of the rotor blades. This makes the yaw loads more susceptible to influences which are small in any other context. Rotor (as opposed to individual blade) modes of vibration will certainly be important in some cases. 
Future wind turbine codes must be capable of modeling additional structural degrees of freedom. This will be difficult because each new turbine design is likely to have different vibratory modes which are important. Codes written for specific modes, such as YawDyn or the current FLAP program, will not be suitable for all designers even if selected additional degrees of freedom are included. On the other hand, full finite element models are not likely to be widely used because of difficulties in creating and interpreting the model, particularly in the preliminary design stage. Versatile structural dynamics codes such as ADAMS, coupied with sophisticated aerodynamics analysis may be the best solution to this problem. This is the subject of future work at the University of Utah.

The aerodynamics analysis has been found to be of great importance in estimation of yaw loads. YawDyn used simplistic representations of aerodynamic phenomena to determine whether those phenomena may be important. Unfortunately, virtually all the aerodynamic effects introduced did play an important role. This means the model must be improved to more accurately account for each of those effects (stall hysteresis, skewed wake corrections and static stall were all important). It is known that delayed static stall occurs on rotor blades, though this effect is not modelled in YawDyn. The Gormont dynamic stall model depends too heavily upon empiricism to be suitable for design of rotors with new airfoils. Thus it is important that new techniques, perhaps borrowed from the helicopter industry, be employed in the wind turbine codes. Dynamic inflow is not modeled (except in a quasi-steady form). The importance of the skewed wake corrections implies that dynamic inflow corrections will also be significant. Implementing these physical phenomena in codes which will be understood and used by designers is a major challenge to the wind industry. 


\section{References}

Berg, D. E., 1983, "Recent Improvements to the VDART3 VAWT Code." Wind/Solar Energy Conference, Kansas City, MO.

Bundas, D. and J. Dugundji, 1981, "Some Experiments on Yaw Stability of Wind Turbines with Various Coning Angles." Massachusetts Institute of Technology, NASA CR-168108.

Butterfield, C. P., 1989a, "Aerodynamic Pressure and Flow-Visualization Measurement from a Rotating Wind Turbine Blade." Eighth ASME Wind Energy Symposium, Houston, TX.

Butterfield, C. P., 1989b, "Three-Dimensional Airfoil Performance Measurements on a Rotating Wing." European Wind Energy Conference, Glasgow, Scotland.

Chaiyapinunt, S. and R. E. Wilson, 1983, "Analysis of Wind Turbines in Yaw." Rocky Flats Wind Energy Research Center, RFP-3678.

Coleman, R. P., A. M. Feingold and C. W. Stempin, 1945, "Evaluation of the Induced Velocity Field of an Idealized Helicopter Rotor." NACA, NACA WRL-126.

Cui, X., A. C. Hansen and N. Siedschlag, 1988, "Yaw Dynamics of Horizontal Axis Wind Turbines: First Annual Report." Solar Energy Research Institute, SERI Technical Report, STR-217-3309.

de Vries, O., 1985, "Comment on the Yaw Stability of a Horizontal-Axis Wind Turbine at Small Angles of Yaw." Wind Engineering. Vol. 9, No. 1. pp.

Gaonkar, G. H. and D. A. Peters, 1986, "Effectiveness of Current Dynamic-Inflow Models in Hover and Forward Flight." Journal of American Helicopter Society. Vol. 31, No. 2. pp.

Gormont, R. E., 1973, "A Mathematical Model of Unsteady Aerodynamics and Radial Flow for Application to Helicopter Rotors." U.S. Army Air Mobility Research and Development Laboratory, USAAMRDL Technical Report, 76-67.

Hansen, A. C. and X. Cui, 1989, "Analyses and Observations of Wind Turbine Yaw Dynamics." ASME Journal of Solar Energy Engineering. Vol. 111, No. 4. pp. 367371.

Hansen, A. C., C. P. Butterfield and X. Cui, 1990, "Yaw Loads and Motions of a Horizontal Axis Wind Turbine." ASME Journal of Solar Energy Engineering. Vol. 112, No. 4. pp. 310-314.

Hansen, A. C. and A. D. Wright, 1991, "A Comparison of Combined Experiment Flap Load Predictions by the FLAP and YawDyn Codes." Tenth ASME Wind Energy Symposium, Houston, TX.

Hoffman, J. A., 1977, "Coupled Dynamics Analysis of Wind Energy Systems." Paragon Pacific, NASA CR-135152. 
Lynette, R., 1988, "California Wind Farms Operational Data Collection and Analysis." Solar Energy Research Institute,

Miller, R. H., 1979, "On the Weathervaning of Wind Turbines." AIAA Journal of Energy. Vol. 3, No. 5. pp.

Pitt, D. M. and D. A. Peters, 1981, "Theoretical Predictions of Dynamic Inflow Derivatives." Vertica. Vol. 5, No. 1. pp.

Prouty, R. W., 1986, Helicopter Performance, Stability and Control. Boston, PWS Publishers.

Savino, J. M. and L. H. Wagner, 1976, "Wind Tunnel Measurements of the Tower Shadow on Models of the ERDA/NASA $100 \mathrm{~kW}$ Wind Turbine Tower." NASA LeRC, DOE/NASA Report No. 1004-77/1.

Shipley, S. A., 1978, "Wind Tunnel Test, WTS Mod-2, Full Span Control, 1/20th Scale." Boeing Aerospace Co., Unpublished report, Code IDENT No. 81205 D27710129-1.

Stoddard, F. S., 1978, "Structural Dynamics, Stability and Control of High Aspect Ratio Wind Turbines." Ph.D. Dissertation, University of Massachusetts.

Stoddard, F. S., 1988, "An Analytical Method for Calculating the Aerodynamic Yaw Moment and Evaluating the Yaw Stability of Horizontal Axis Wind Turbines." Seventh ASME Wind Energy Symposium, New Orleans, LA.

Swift, A. H. P., 1981, "The Effects of Yawed Flow on Wind Turbine Rotors." PhD. Dissertation, Washington University.

Viterna, L. A. and R. D. Corrigan, 1981, "Fixed-Pitch Rotor Performance of Large HAWTs." DOE/NASA Workshop on Large HAWTs, Cleveland, $\mathrm{OH}$.

Wilson, R. E. and P. B. S. Lissaman, 1974, "Applied Aerodynamics of Wind Power Machines." Oregon State University, NTIS PB 238594.

Wolfram, S., 1991, Mathematica: a system for doing mathematics by computer. Redwood City, CA, Addison-Wesley Publishing Co.

Wright, A. D., M. L. Buhl and R. W. Thresher, 1987, "FLAP Code Development and Validation." SERI, TR-217-3125.

Wright, A. D., R. W. Thresher and C. P. Butterfield, 1991, "Status of a Teetered Rotor Code Development and Validation Project." Tenth ASME Wind Energy Symposium, Houston, TX.

Young, J., A. C. Hansen and P. B. Anderson, 1988, "Implications of Unmatched Blade Pitch and Twist in 3-bladed Rigid Rotors." Windpower '88, Honolulu, Hawaii.

\section{Bibliography of publications resulting from this research:}

Cui, X., 1988, "Yaw Dynamics of Horizontal Axis Wind Turbines." Master of Science Thesis, University of Utah. 
Cui, X., A. C. Hansen and N. Siedschlag, 1988, "Yaw Dynamics of Horizontal Axis Wind Turbines: First Annual Report." Solar Energy Research Institute, SERI Technical Report, STR-217-3309.

Dorius, T., 1992, "Yaw Aerodynamics of Horizontal Axis Wind Turbines." Master of Science Thesis, University of Utah.

Hansen, A. C., 1988, "A Method for Analyzing Wind Turbine Dynamic Response Test Data." Journal of Solar Energy Engineering. Vol. 110, No. 4. pp. 335-339.

Hansen, A. C., C. P. Butterfield and X. Cui, 1990, "Yaw Loads and Motions of a Horizontal Axis Wind Turbine." Journal of Solar Energy Engineering. Vol. 112, No. 4. pp. 310-314.

Hansen, A. C. and X. Cui, 1988, "Analyses and Observations of Wind Turbine Yaw Dynamics." Seventh ASME Energy Technology Conference, New Orieans, LA.

Hansen, A. C. and X. Cui, 1989, "Analyses and Observations of Wind Turbine Yaw Dynamics." ASME Journal of Solar Energy Engineering. Vol. 111, No. 4. pp. 367371.

Hansen, A. C. and X. Cui, 1989, "Recent Progress in the Prediction of Yaw Dynamics." Eighth ASME Wind Energy Symposium, Houston, TX.

Hansen, A. C. and X. Cui, 1989, "Yaw Dynamics of Horizontal Axis Wind Turbines: Second Annual Report." Solar Energy Research Institute, SERI Technical Report, STR-217-3476.

Hansen, A. C. and A. D. Wright, 1991, "A Comparison of Combined Experiment Flap Load Predictions by the FLAP and YawDyn Codes." Tenth ASME Wind Energy Symposium, Houston, TX.

Schnepp, R., 1991, "Wind Turbine Aerodynamic Loads from Blade Bending Moments." Master of Science Thesis, University of Utah.

Siedschlag, N., 1987, "Yaw Dynamics of Horizontal Axis Wind Turbines." Master of Science Thesis, University of Utah. 


\title{
Appendix A
}

\author{
Derivation of the Equations of Motion
}

\section{Major Assumptions}

It is necessary to make a number of assumptions to keep the model simple enough to satisfy all of the project objectives. The more important assumptions are listed below.

\section{Structural dynamics}

- There are a maximum of $\mathrm{B}+1$ degrees of freedom. $\mathrm{B}$ is the number of blades. The teetering rotor system has only two degrees of freedom (teeter and yaw).

- Yaw angles can be arbitrarily large.

- Flap angles remain small such that these approximations are valid: $\operatorname{Sin} \beta=\beta, \cos \beta=1$.

- The blades are completely rigid. All flap motion is confined to rotation about the flap hinge axis. This axis remains in the plane of rotation of the rotor. That is, the pitch and twist angles of the blade are neglected when considering the blade deflections. (Pitch and twist are not neglected in the aerodynamic analysis.)

- Blade lead-lag (in-plane) vibrations are neglected.

- Blade torsional vibrations are neglected.

- The blades are slender such that the mass moments of inertia about the flap "hinge" or the edgewise root are equal and the moment of inertia about the pitch axis is negligible. $\left(\mathrm{I}_{\mathrm{b}}=\mathrm{I}_{\mathrm{L}}, \mathrm{I}_{\theta}=0\right)$

- Each blade has identical mass properties and the blades are placed at equally spaced azimuth intervals. However, the pitch angle of each blade can be specified independently.

- The tower is completely rigid and vertical. That is, there is no motion or tilt of the top of the tower.

- The rotor axis intersects the tower (yaw) axis. There is no rotor offset but there may be rotor tilt. The tilt angle is small such that standard small angle assumptions can be applied.

- When the rotor has a teetering hub, the blades are competely rigid and coupled such that the flap rate of blade \#1 is the negative of the flap rate of blade \#2. The flap angles differ in sign and by an offset (if there is rotor preconing).

\section{Aerodynamics}

- Blade element/momentum methods are used to determine "basic" induced velocities.

- The basic induced velocities are corrected for skewed wake effects but not unsteady aerodynamic effects.

- The Viterna flat-plate model is used to estimate static lift and drag coefficients at high angles of attack.

- The Gormont dynamic stall method is applied to find the lift coefficient. Drag coefficients are the static, two-dimensional values.

- A digital filter is used to "smooth" the angle of attack history for estimation of rate of change of angle of attack. The filtered angle of attack is not used in any other calculation.

- The same airfoil data is used for the entire blade. Thus thickness, Reynolds number and changing airfoil section effects are not considered. 
The equations were derived using Euler's Equations and the more general, threedimensional rigid body equations. Though this method tequires extensive algebraic manipulation, it also provides other information of value which is not readily available from Lagrange's method. The blade accelerations and blade root reaction forces are determined in the course of the derivation and useful elsewhere.

The commercially-available symbolic manipulation program called Mathematica $($ ) was used in all of the derivations. This program makes the extensive algebraic manipulations relatively simple and immune from human error. It also provides a convenient, if somewhat unconventional, method of documenting the derivation. The sections that follow were copied directly from the data files ("notebooks") used in the Mathematica calculations. They are quite simple to read and understand and provide a complete record of the assumptions and manipulations required to generate all of the equations of motion. 


\section{Acceleration of the blade center of gravity.}

The result of this calculation is used in subsequent derivations of the equations of motion. The text below, in Courier font, is a direct copy of the inputs and outputs of the Mathematica® program. Readers should consult the Mathematica reference book for details of the command syntax. However, most of the Mathematica language is apparent without referring to the Mathematica manuals.

All text enclosed in $\left({ }^{*} \ldots{ }^{*}\right)$ is a "comment" line for annotation of the Mathematica program. Braces $\{\ldots, \ldots, \ldots\}$ enclose components of a vector (though this is not the exclusive use of braces). All vectors are in Cartesian coordinate systems as identified in the comment sections of the program and the figures of Section 3.0.

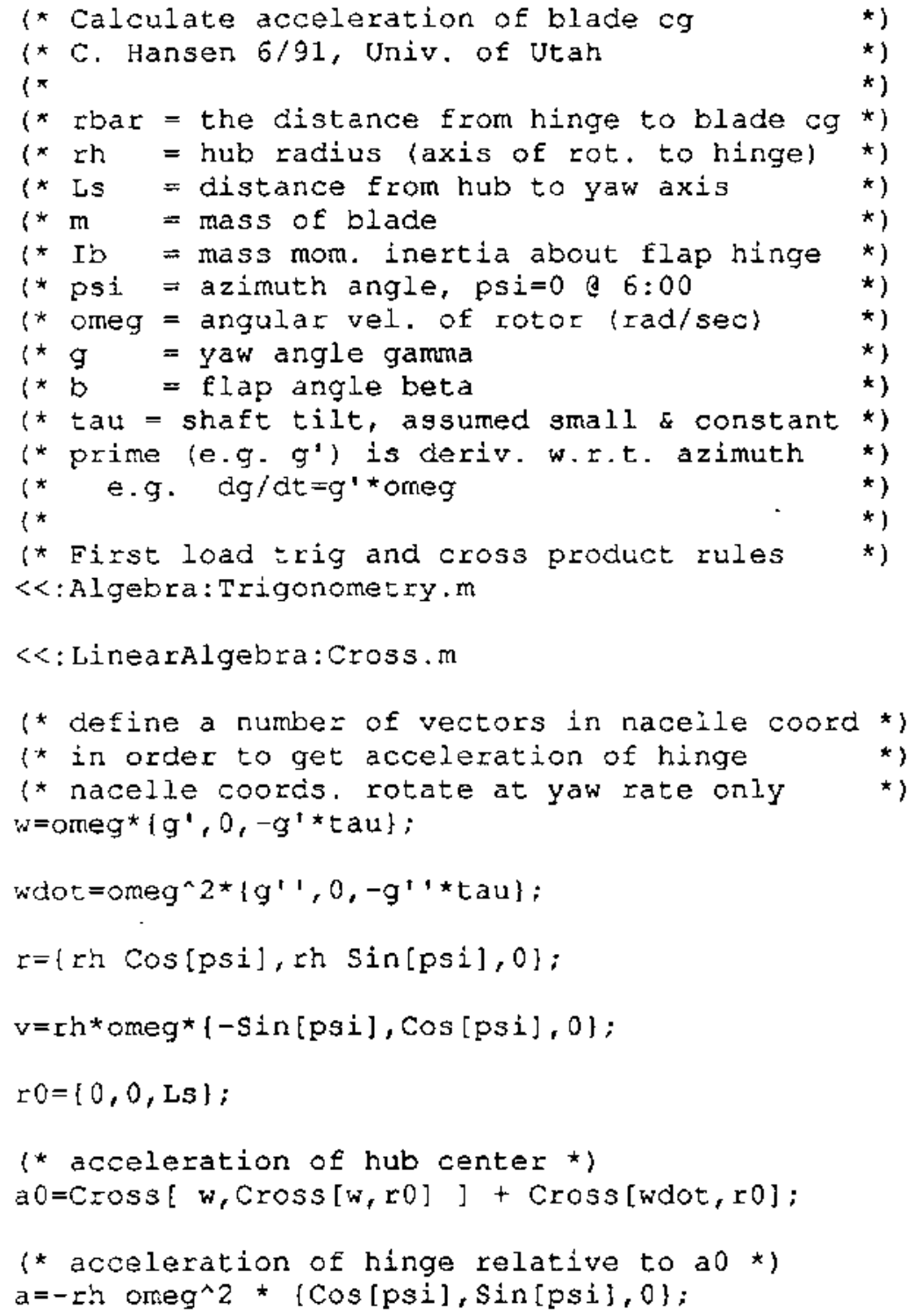




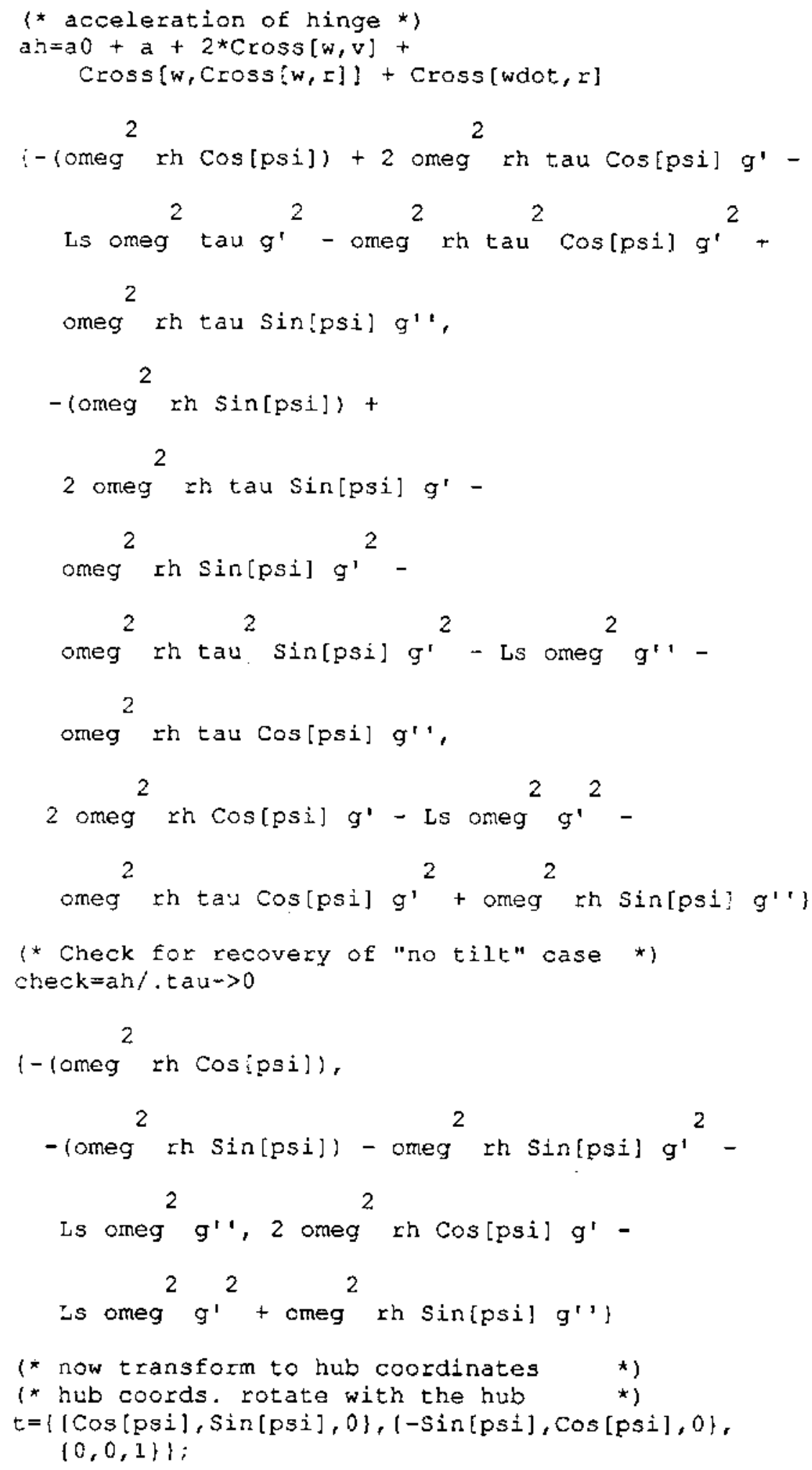




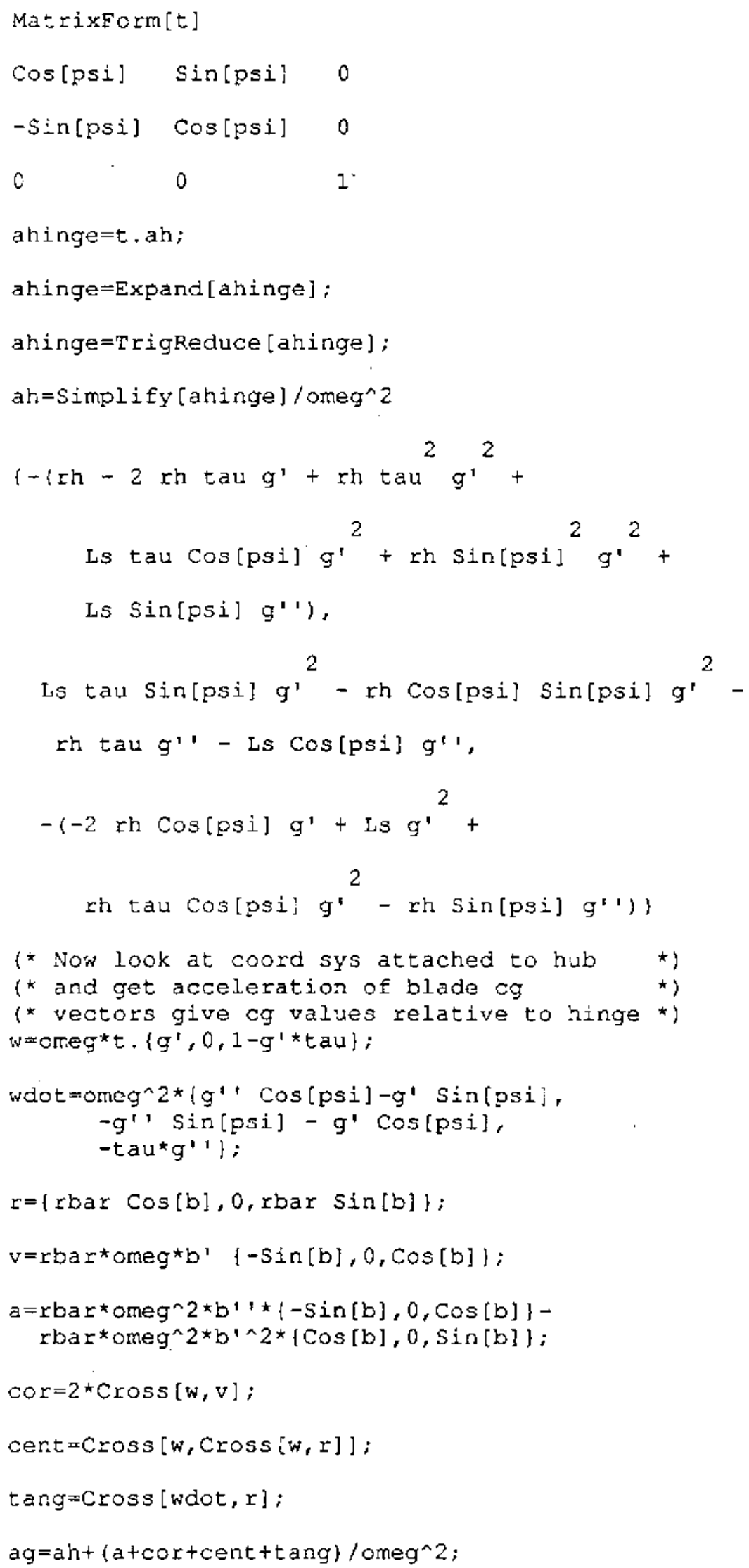




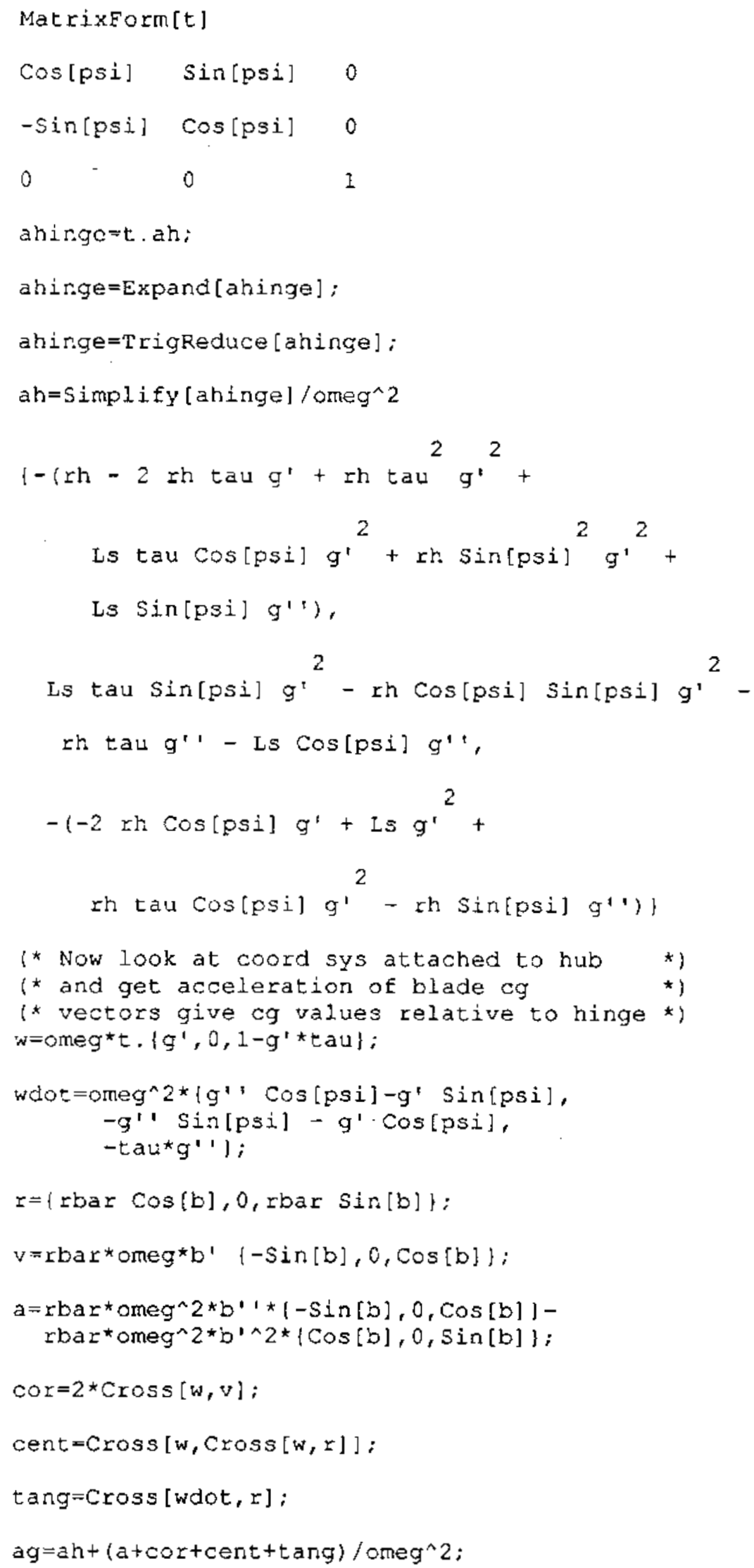




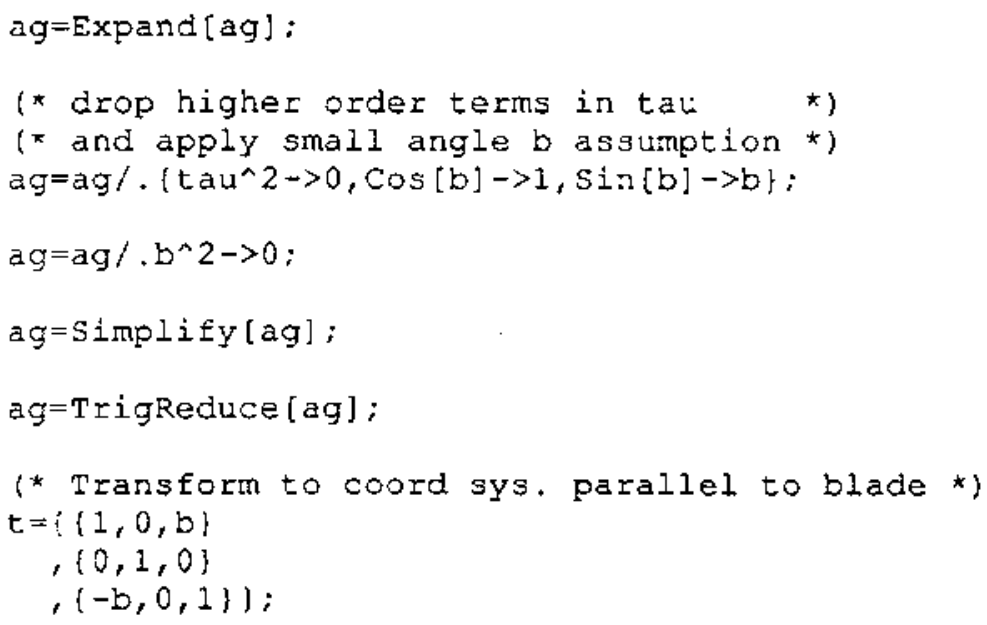


ag

l- rbar + rh -2 rbar tau $g^{\prime}-2$ rh tau $g^{\prime}$ -

2 b rbar cos[psi] $g^{\prime}-2$ b rh cos[psi] $g^{\prime}+$

2 rbar $\sin [p s i] b^{\prime} g^{\prime}+$ Ls b $g^{{ }^{2}}+$

Ls tau $\cos [p s i] g^{2}+\operatorname{rbar} \sin [p s i]^{2} g^{\prime}+$

$r h \sin \left[p s i{ }^{2} g^{\prime 2}+L s \sin [p s i] g^{\prime \prime}-\right.$

b rh $\sin$ (psi] g''),

- (2 rbar cos[psi] b' $g^{\prime}-$ Ls tau sin[psi] $g^{\prime}+$

rbax $\cos [p s i] \sin [p s i] g^{2}+$

rh $\cos [p s i] \sin [p s i] g^{\prime 2}+\operatorname{rbar} t a u g^{\prime \prime}+$

rh tau g' ${ }^{\prime \prime}+$ Ls cos[psi] g' ${ }^{\prime}+$

b rbar cos[psi] g''),

b rbar $+b$ rh +2 rbar Cos[psi] $g^{\prime}+$

$2 \operatorname{rh} \cos [p s i] g^{\prime}-\operatorname{Ls} g^{\prime 2}-b \operatorname{rbar} g^{2}-$

rbar tau $\cos [p s i] g^{\prime 2}-x h \operatorname{tau} \cos [p s i] g^{\prime}+$

$b \operatorname{rbar} \sin [p s i]^{2} g^{\prime 2}+b \operatorname{rh} \sin [p s i]^{2} g^{\prime}+$

rbar $b^{\prime \prime}+$ Ls b sin[psi] $g^{\prime \prime}+$

rbar Sin[psi] g''+rh Sin[psi] g''] 


\section{Flap Equation of Motion}

This Mathematica file derives the flap equation of motion. It employs the result of the previous calculation of the acceleration of the blade c.g.

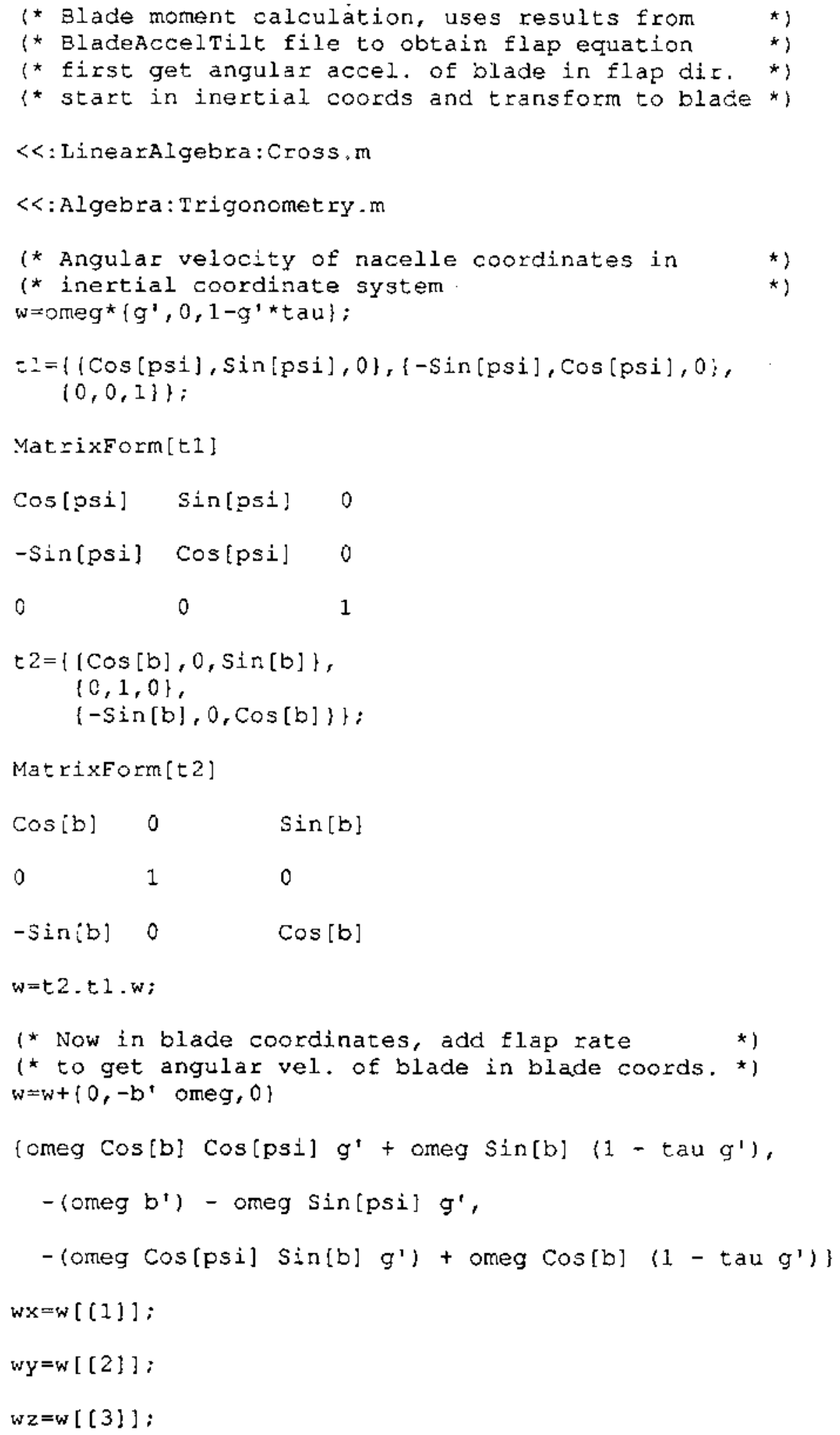




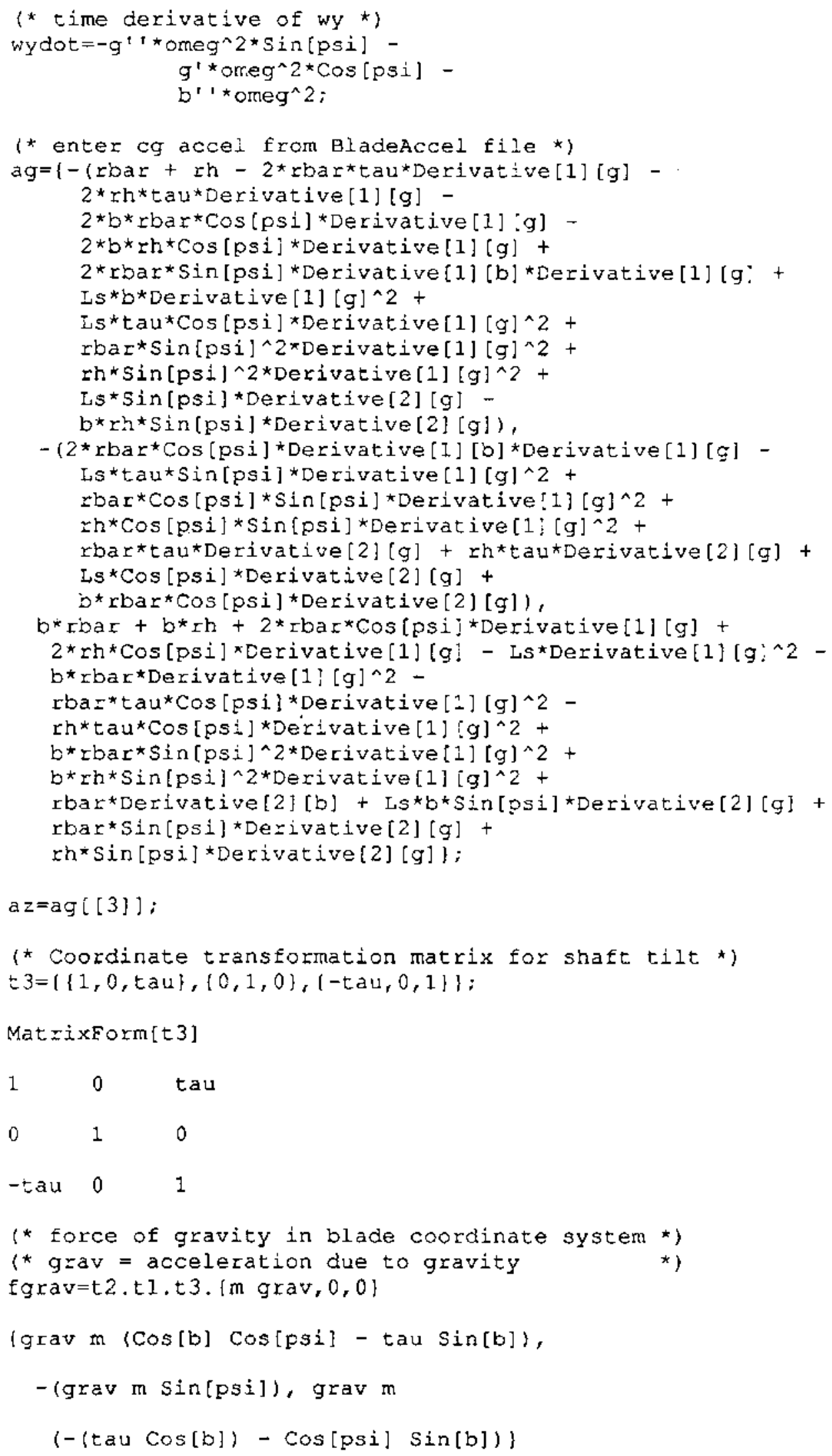




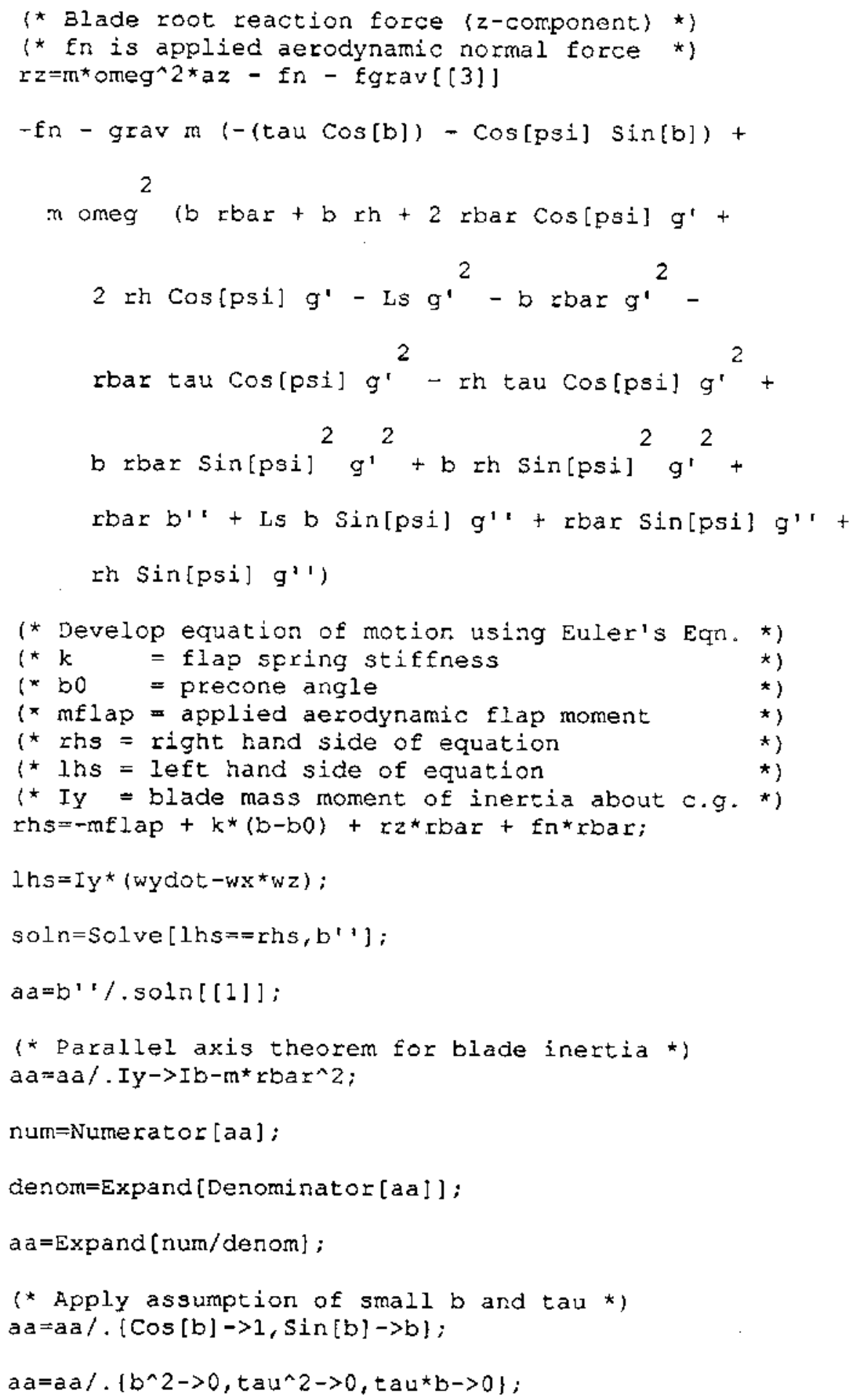




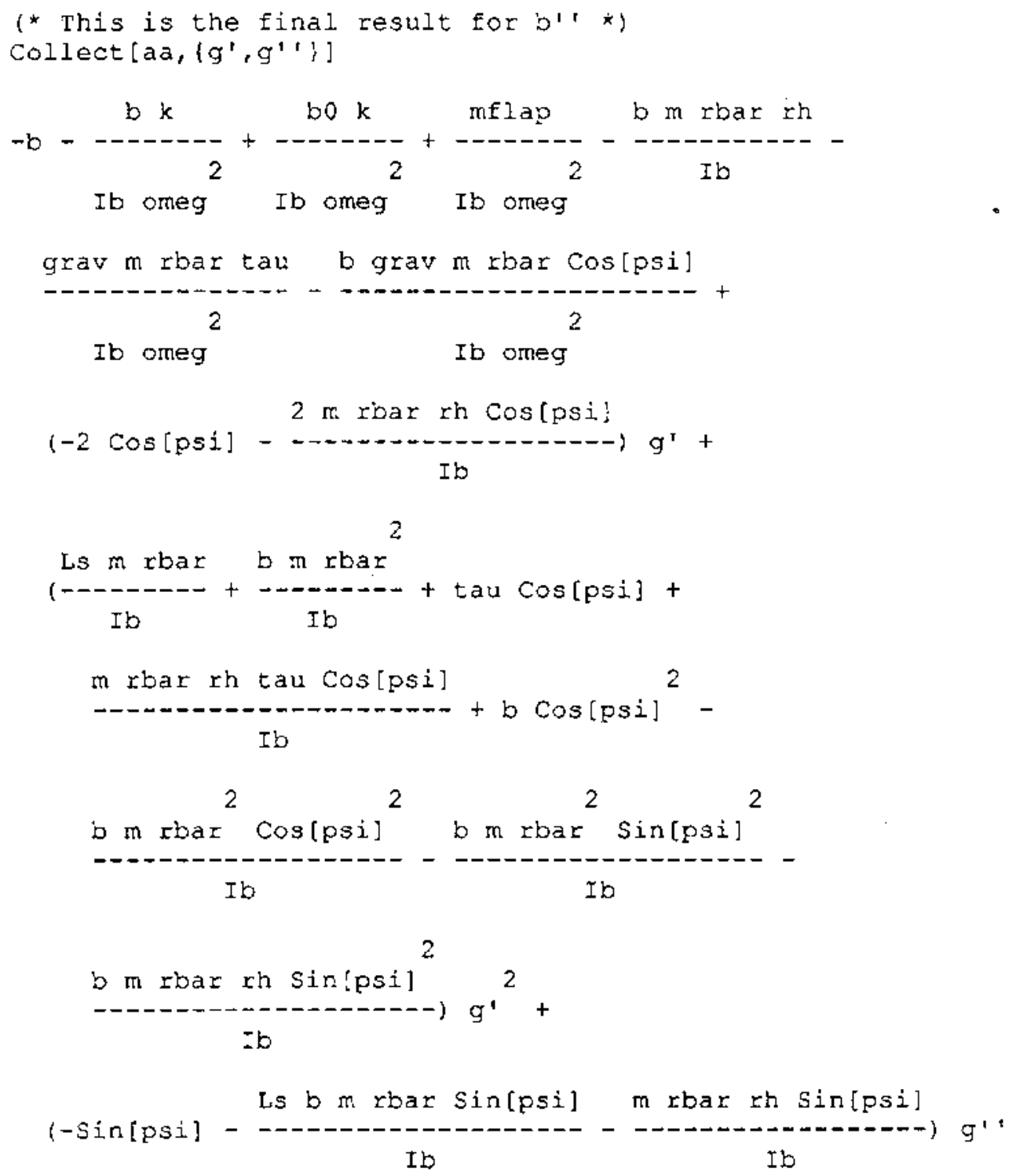


(* Check for tilt->0 *)

check $=$ Collect $\left[a a,\left(g^{\prime}, g^{\prime}\right)\right] / . t a u m>0$

$$
\begin{array}{cccc}
b k & b 0 k & \operatorname{mflap} & b \mathrm{~m} \mathrm{rbax} r h \\
-b- & 2 & 2 & \text { Ib }
\end{array}
$$

b grav m rbar cos [psi]

$$
2
$$

Ib omeg

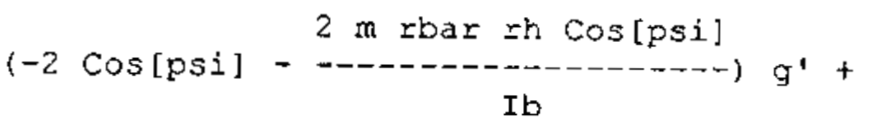

2

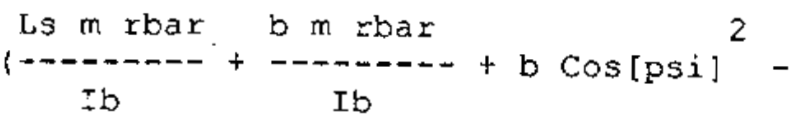

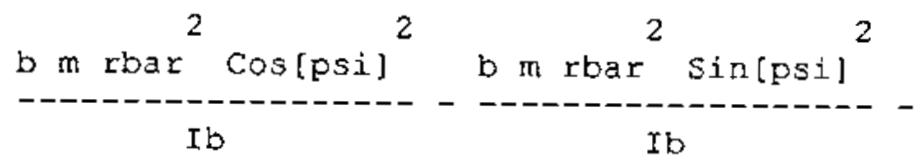

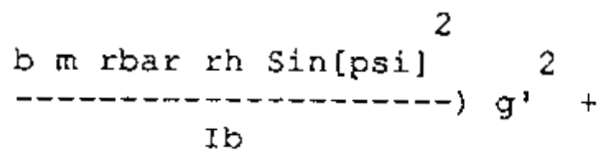

Ib

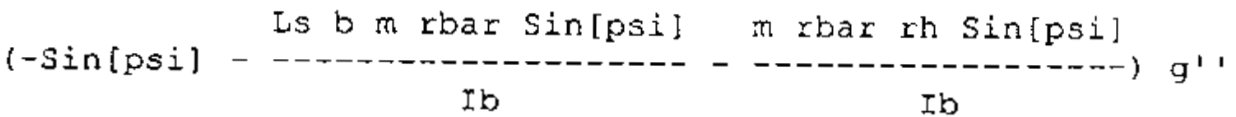




\section{Yaw Equation of Motion (Without Flap)}

In this section the yaw equation of motion is derived for the case when there is no blade flap motion. The flap rate is zero and the flap angle equals the precone angle. The blade root spring has infinite stiffness and is not used in this derivation.

The result obtained in this section applies to one blade. The complete yaw equation for an arbitrary number of blades is found by summing the equations for single blades (at the appropriate azimuth angles). The resulting equations are presented in Section 3 of this report.

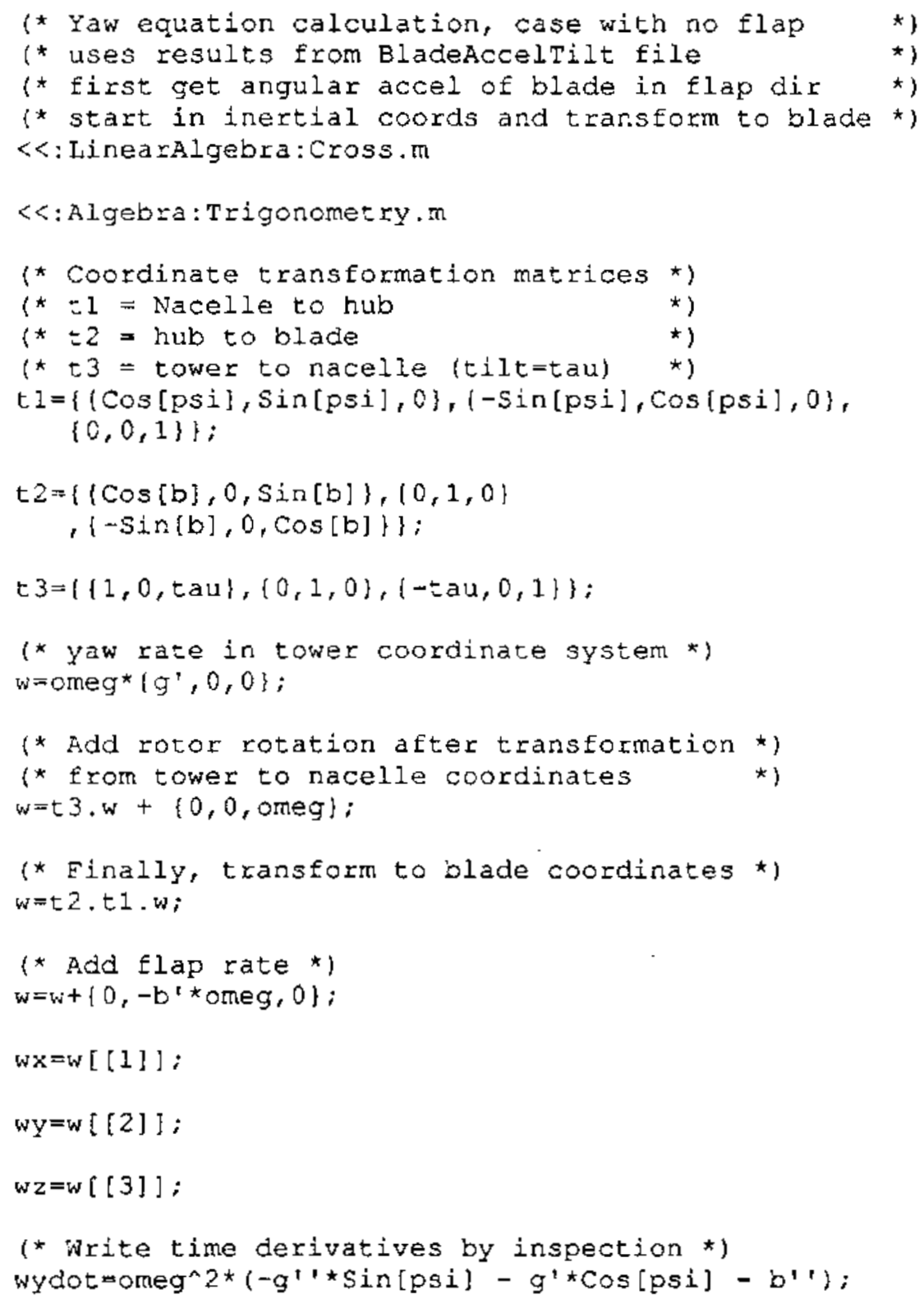




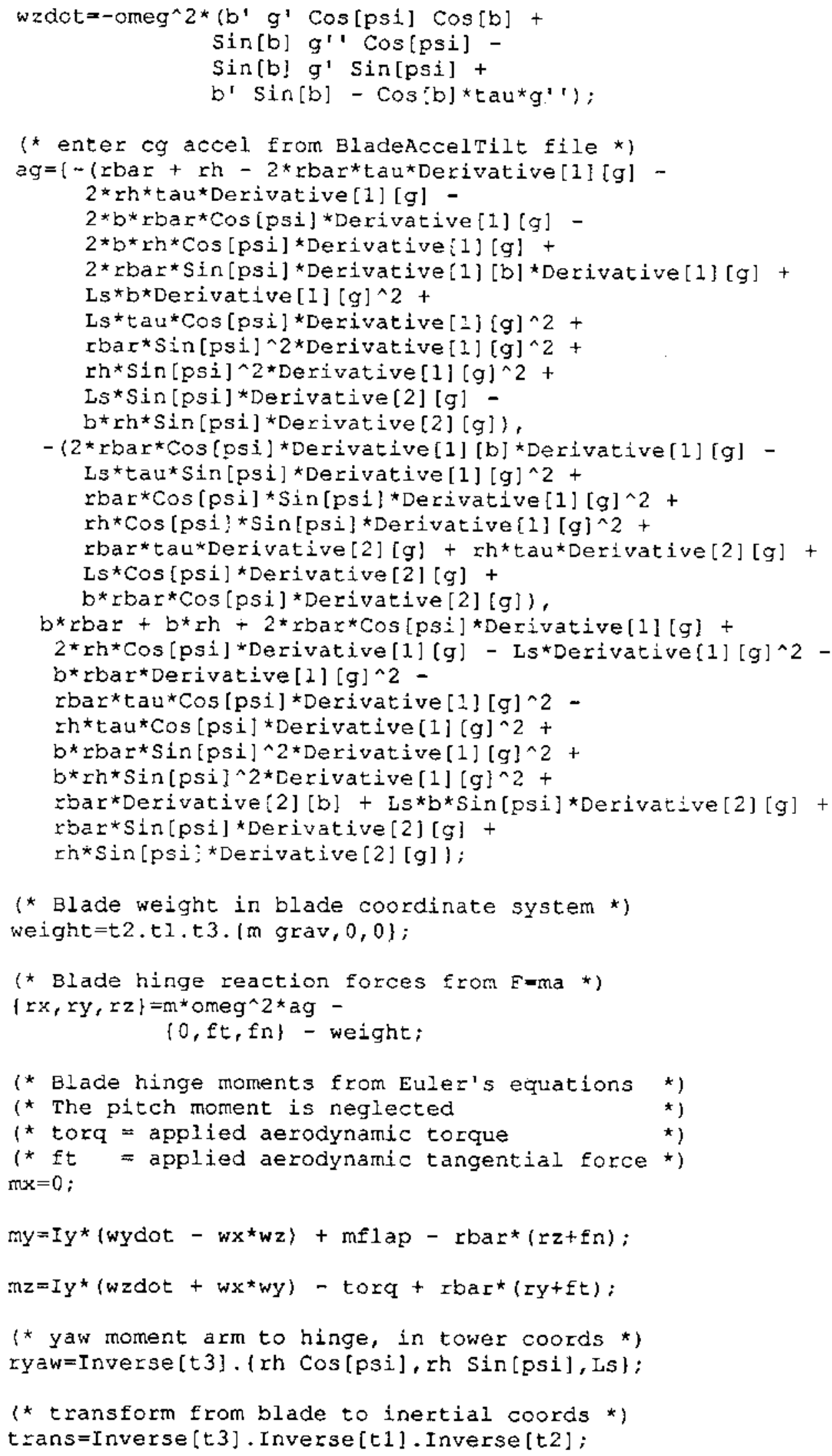




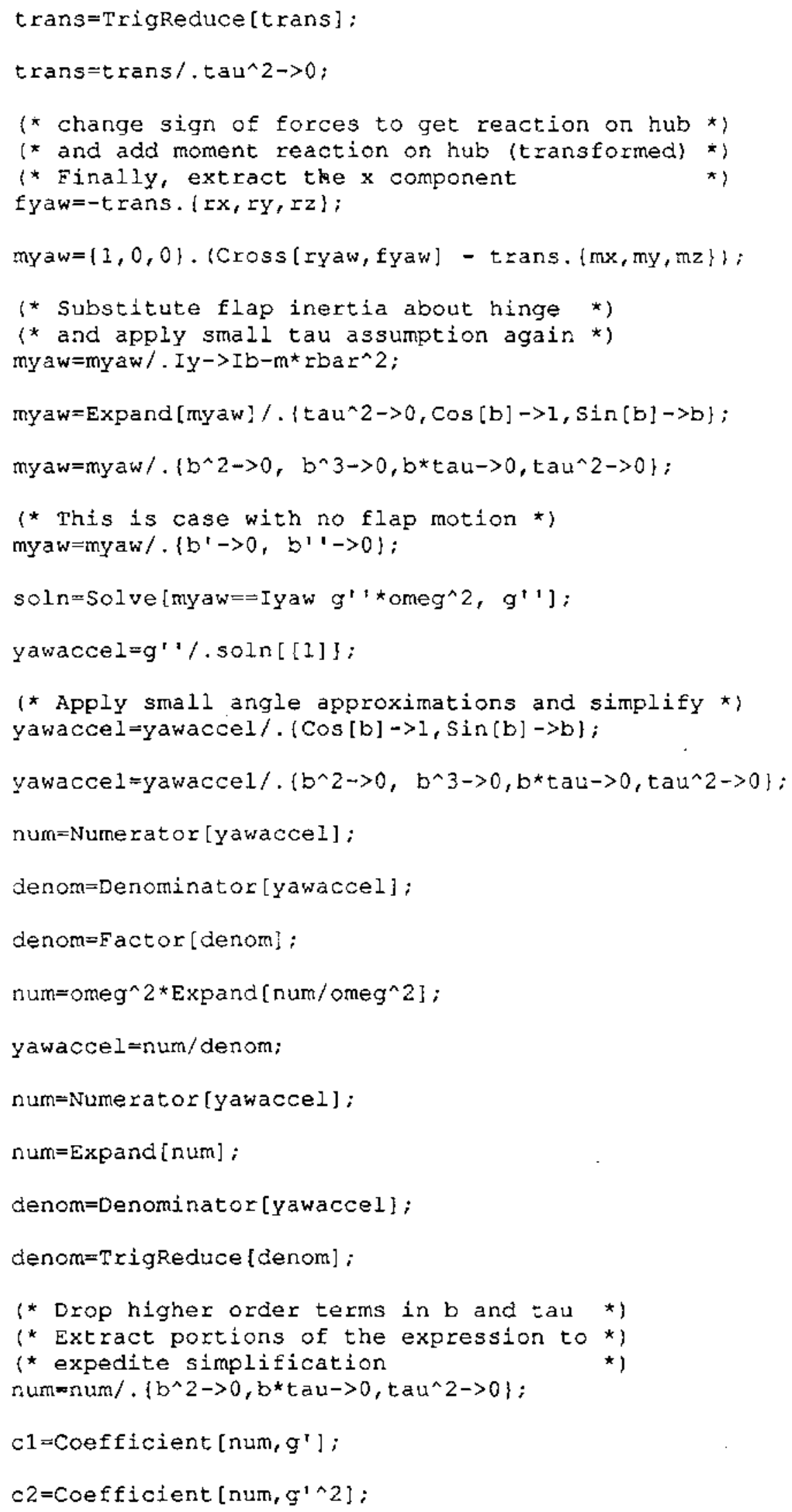




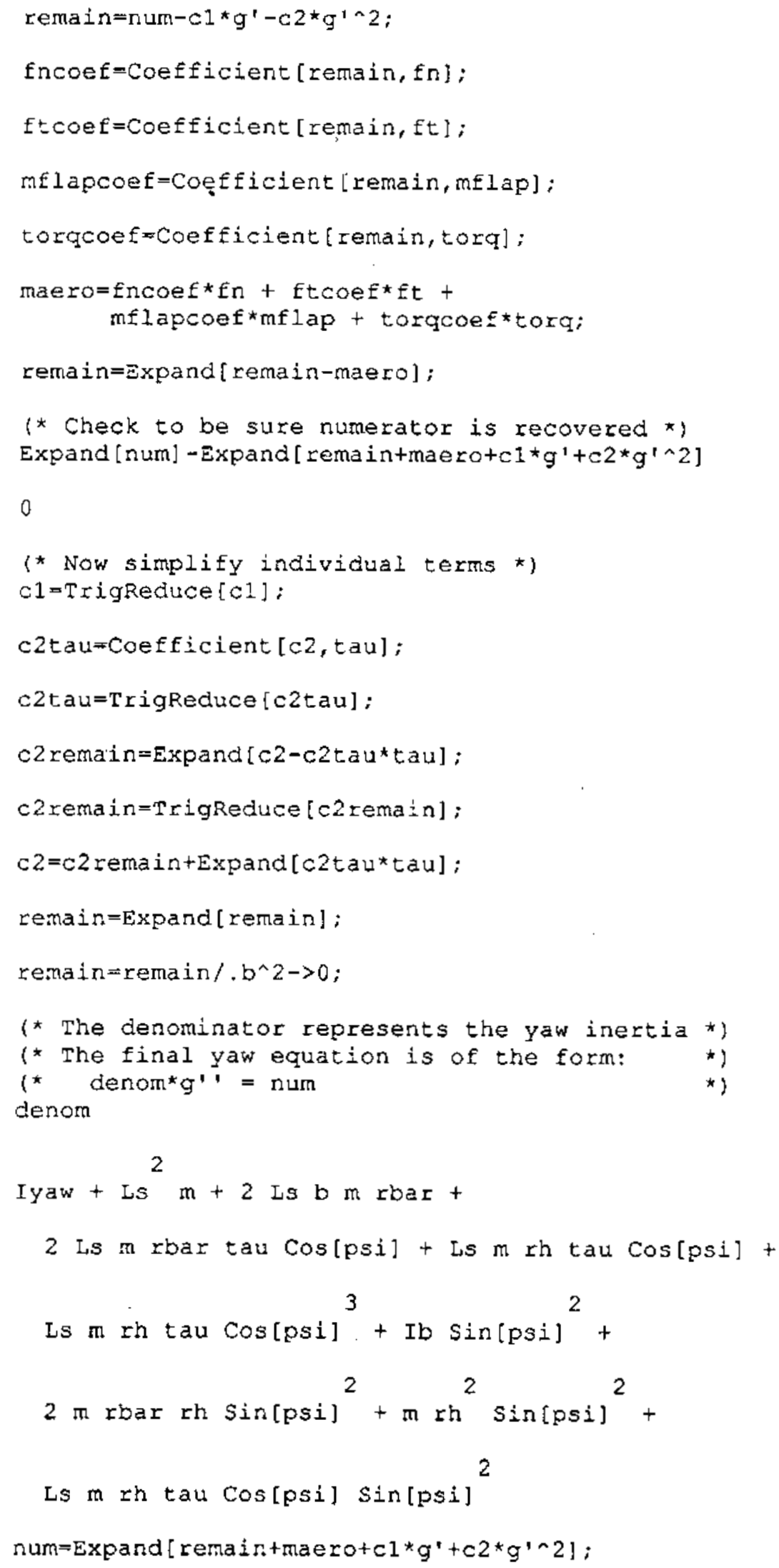




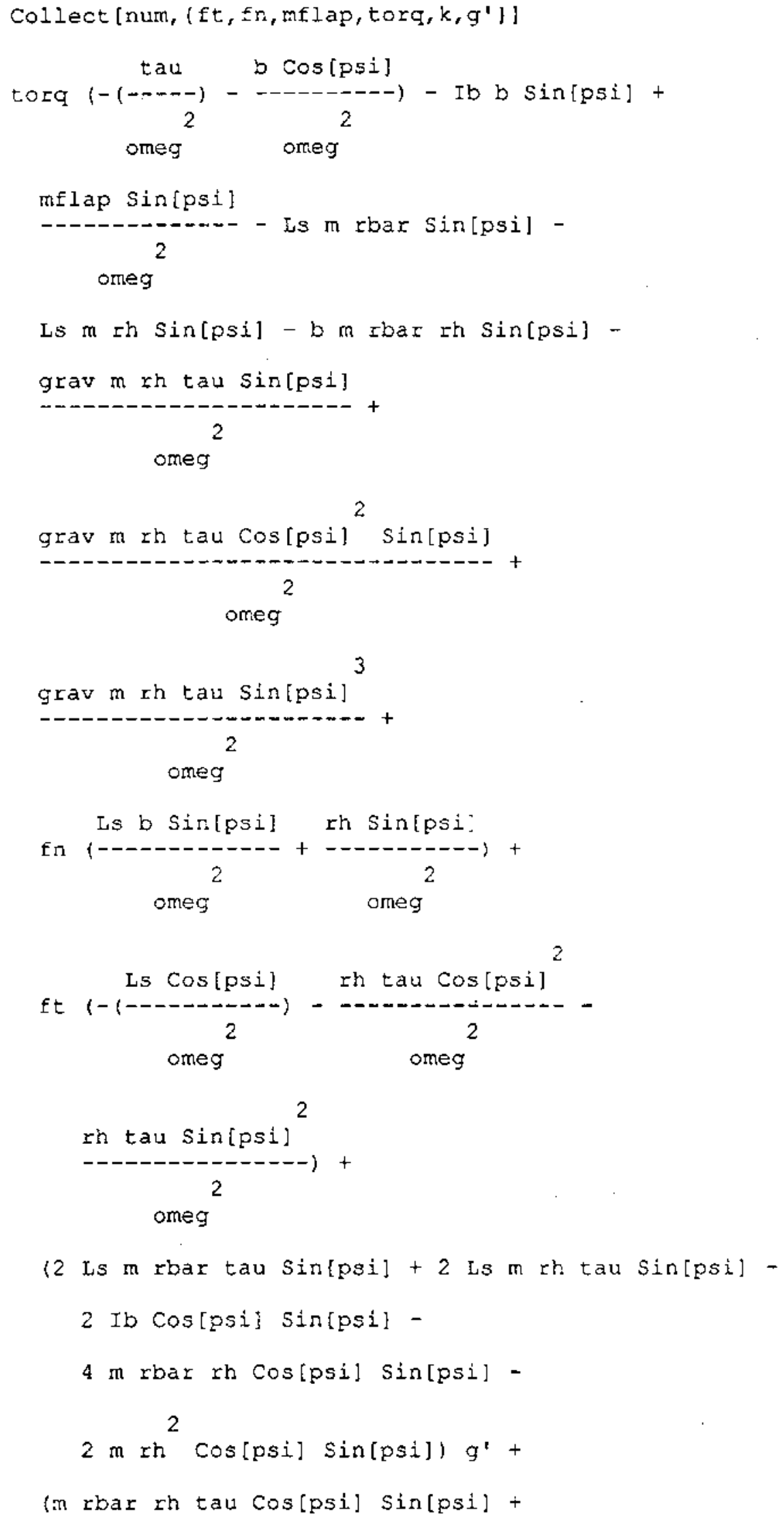




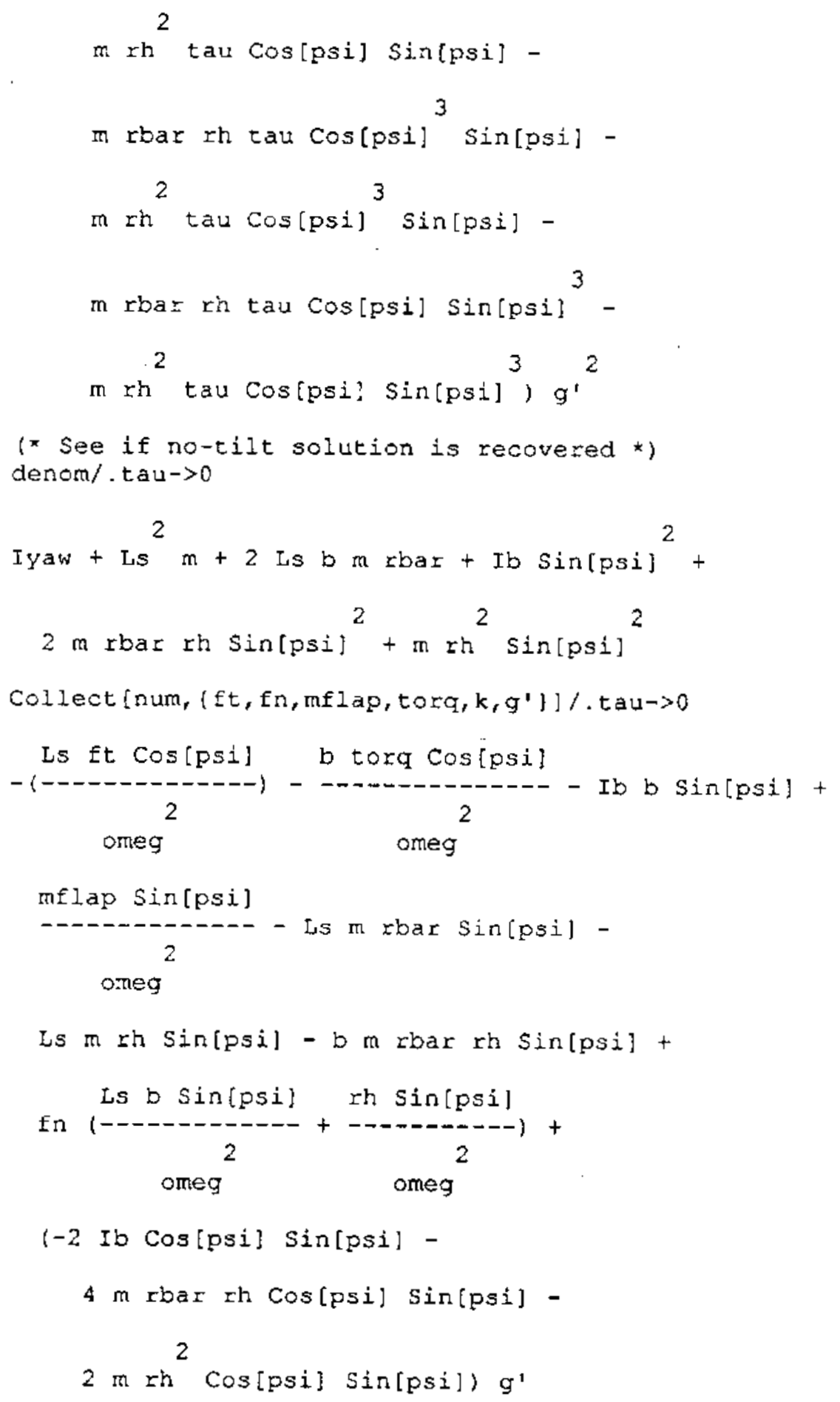




\section{Yaw Equation of Motion (Including Flap DOF)}

This section derives the fully coupled yaw equation of motion for the rigid hub, flapping rotor. Terminology and coordinate systems are the same as used in previous Mathematica files. As in the previous derivation, the complete yaw equation of motion is obtained by summing the result of this section over all blades.

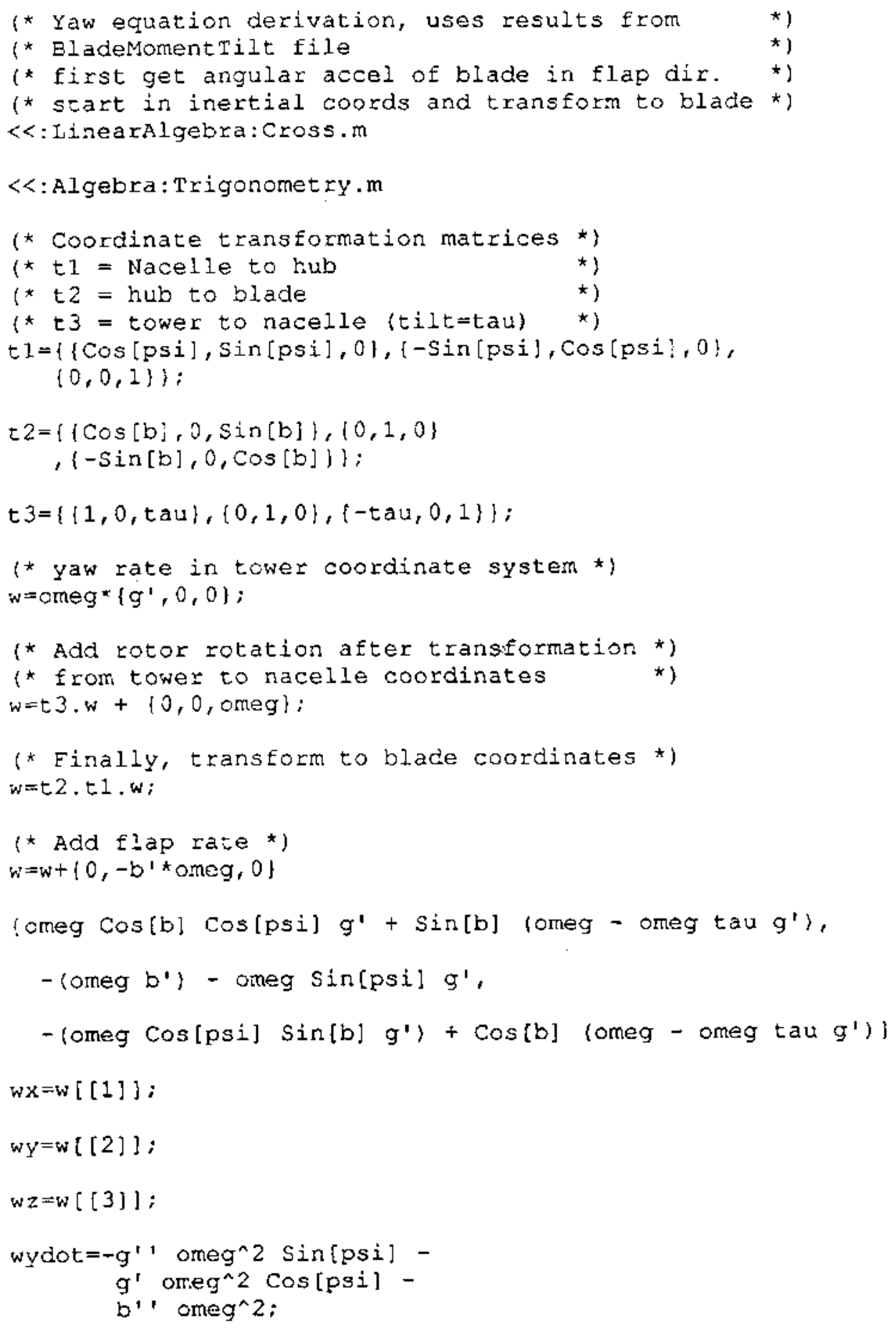




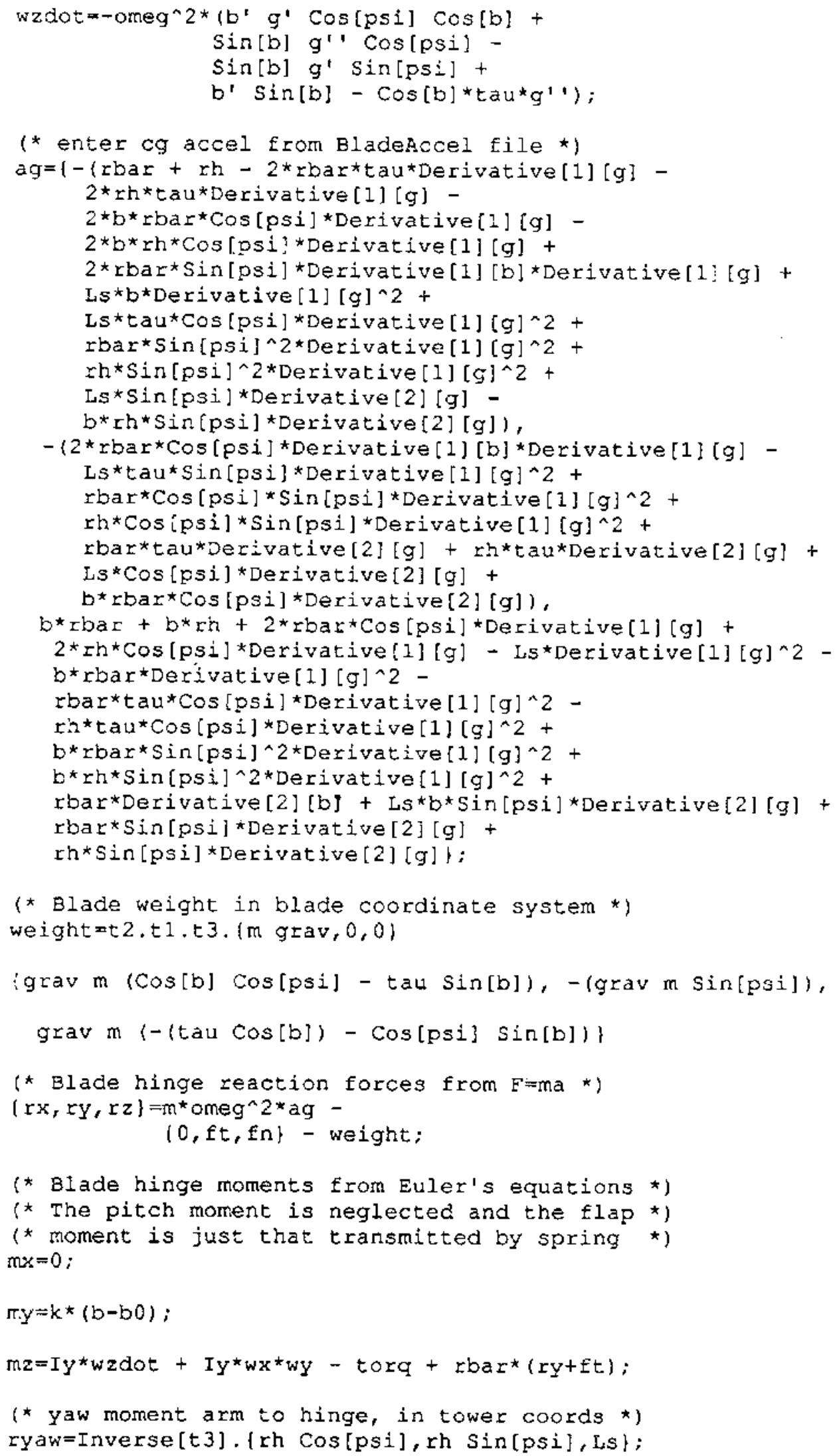




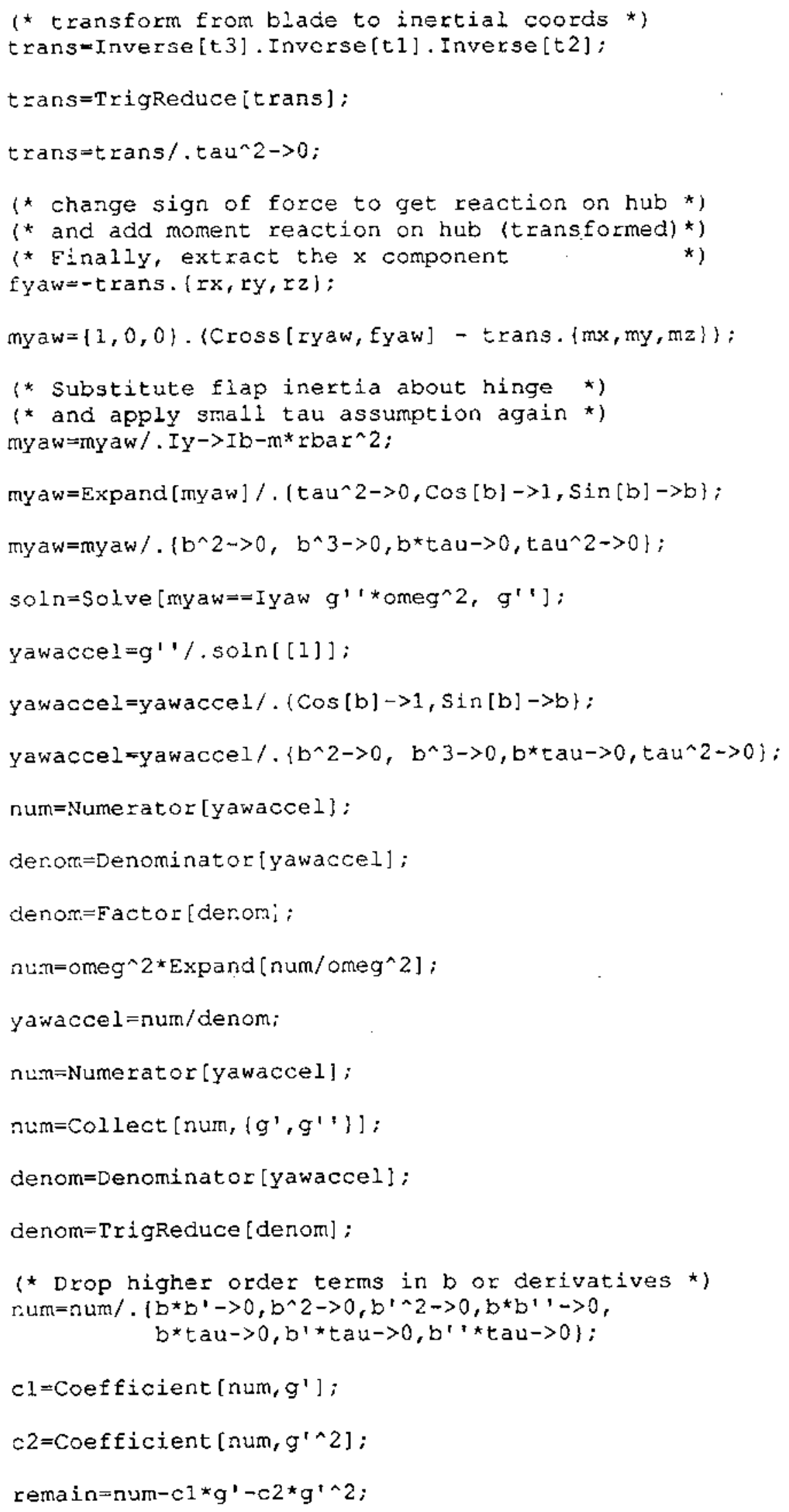




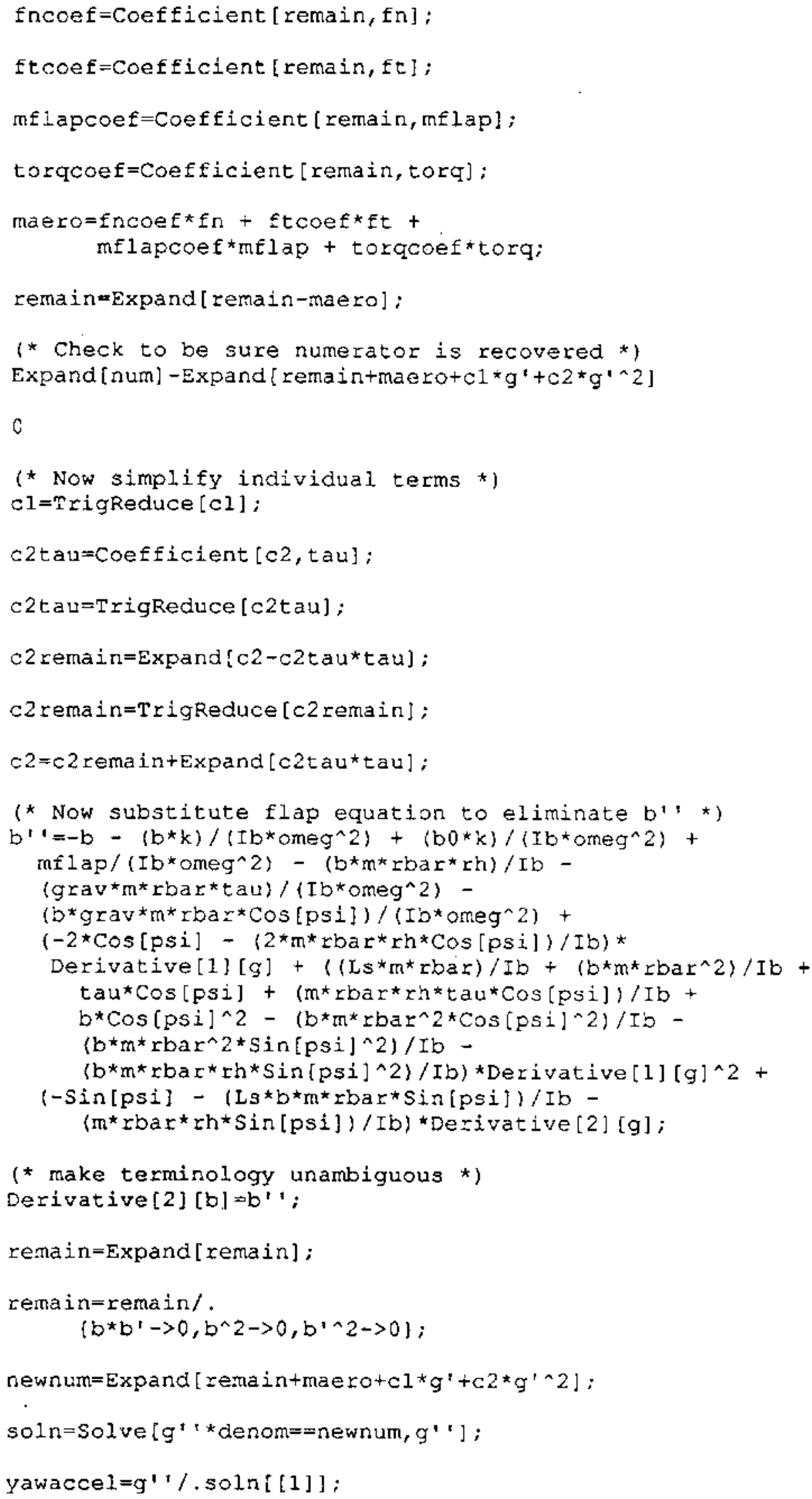




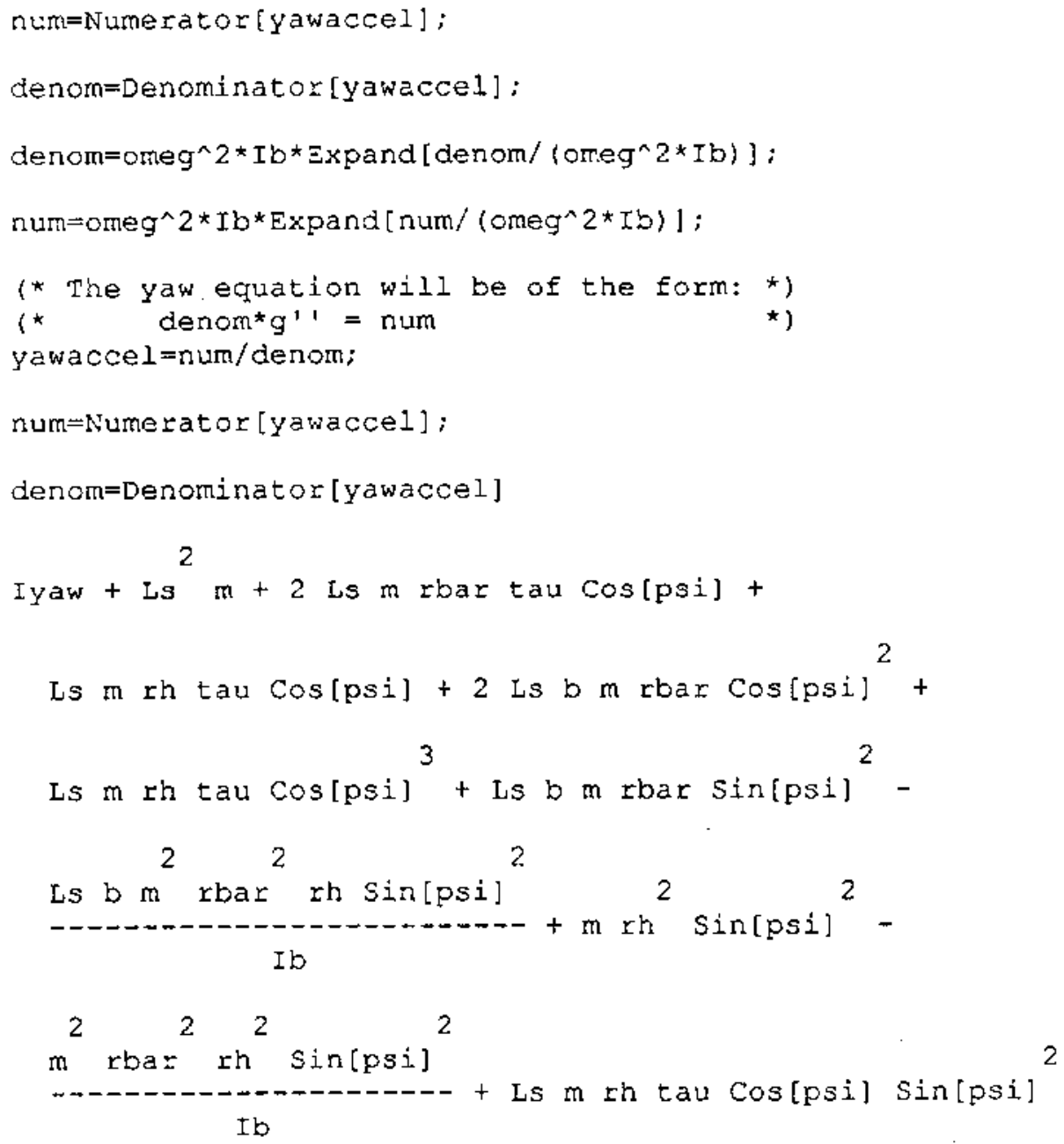




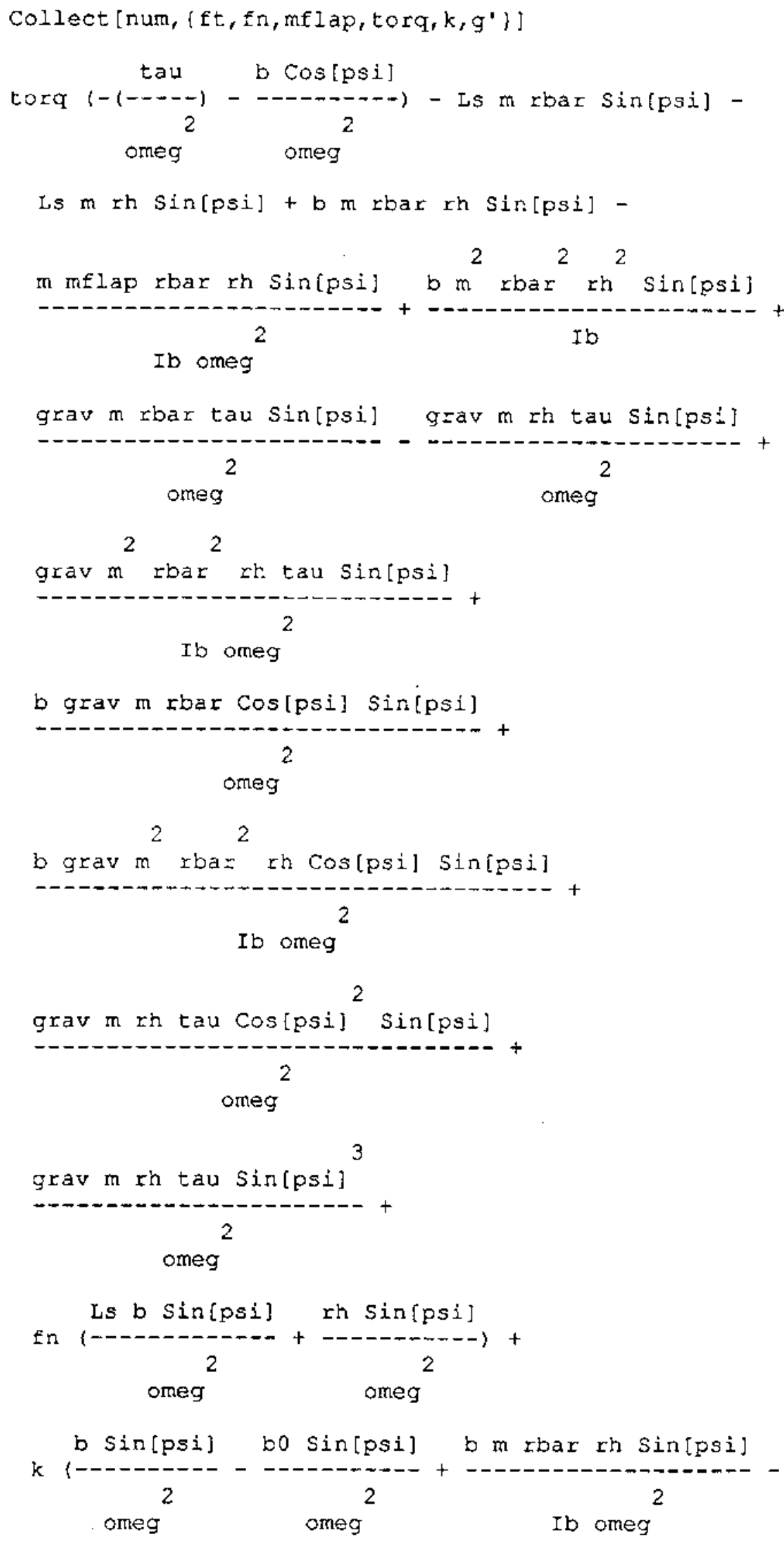


bo $m$ rbar rh $\sin [p s i]$
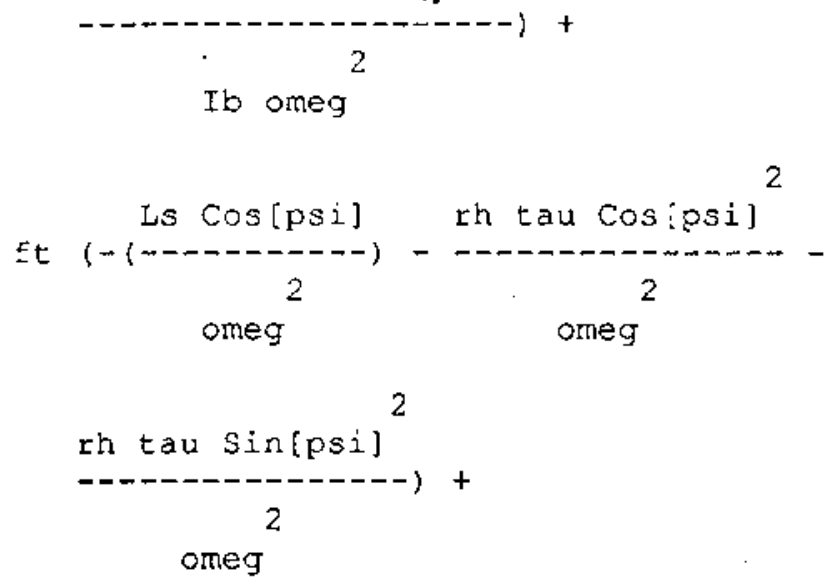

(2 Ls m rbar tau Sin[psi] + 2 Ls $\pi$ rh tau Sin[psi) -

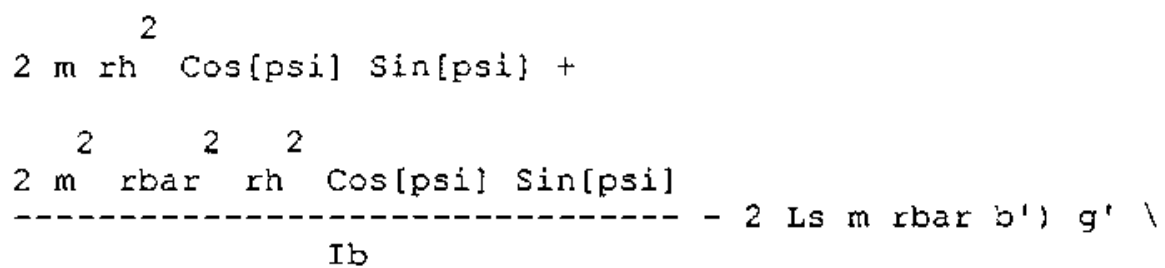




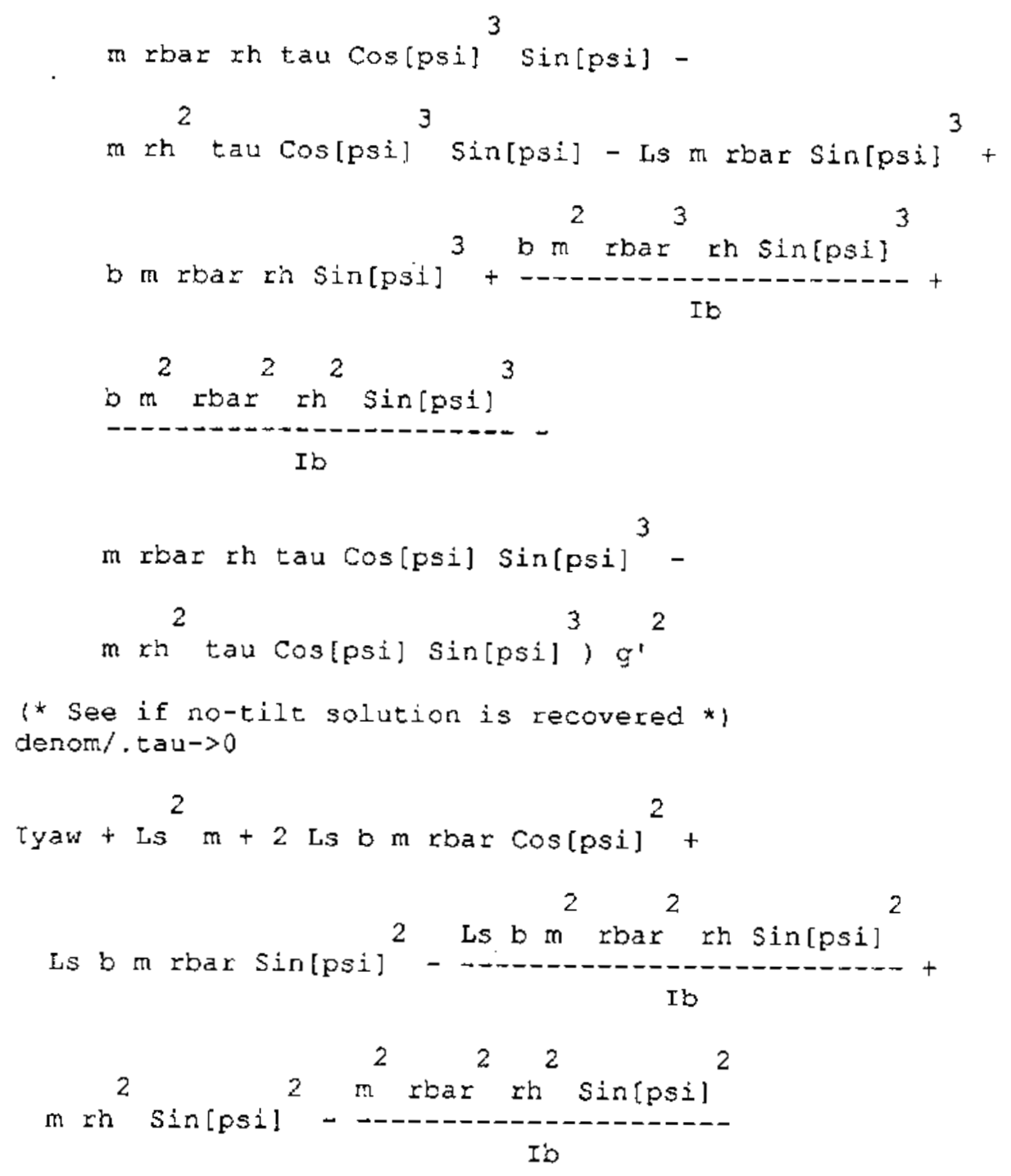




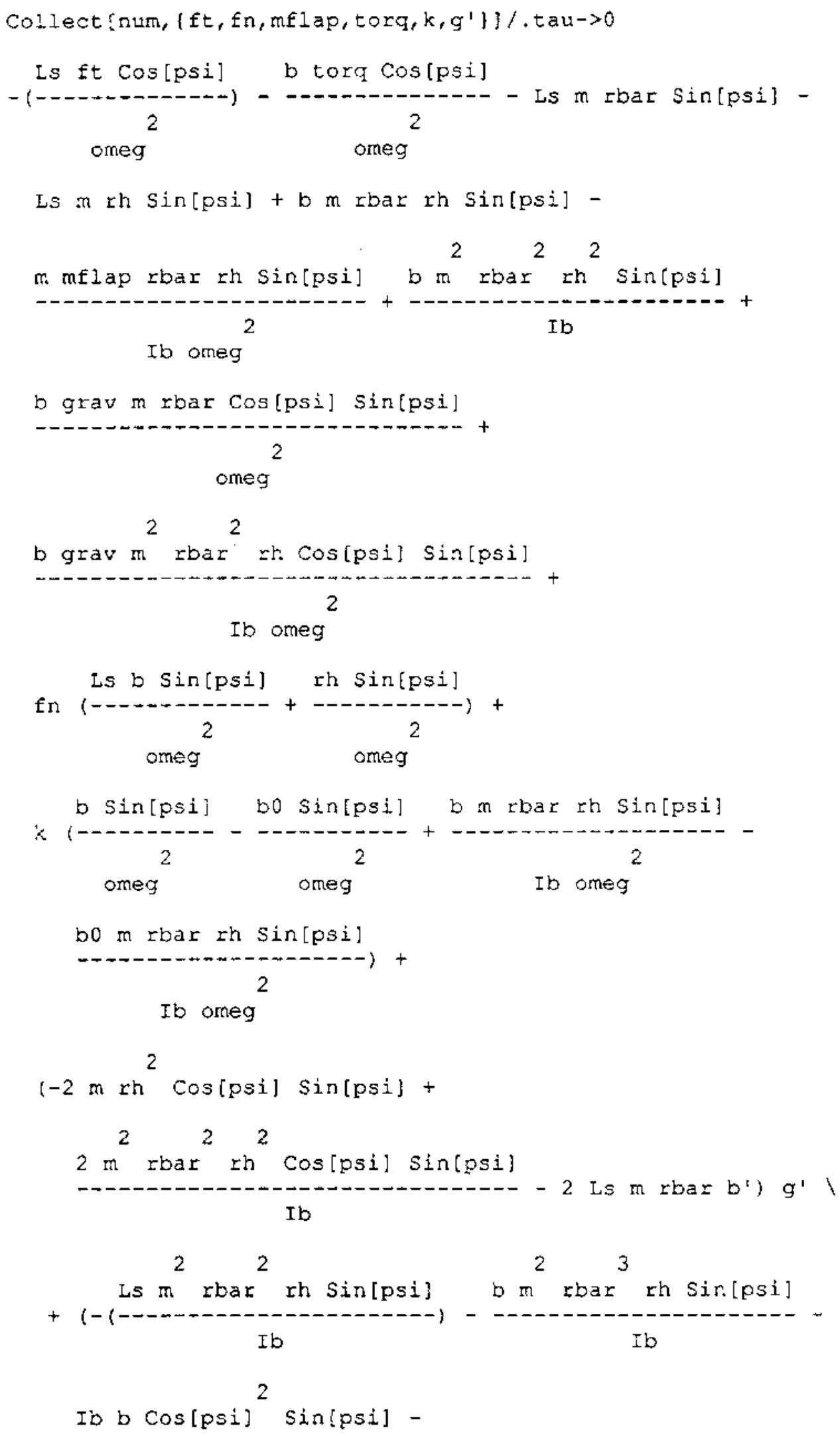




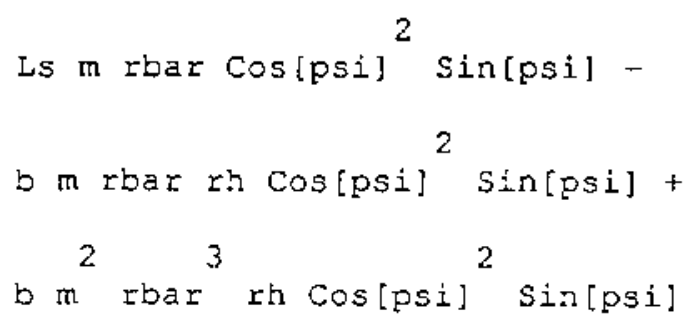




\section{Teetering Rotor Equations}

This section derives the yaw and teetering equations of motion for a simple teetering rotor. The blade is completely rigid and connected to the shaft with an idealized teeter hinge, with linear springs and dampers. The center of mass of the rotor is offset from the teeter axis by a length "sl", the undersling. This equation applies only to two bladed rotors and is complete as presented at the end of the derivation. To maintain consistency with previous equations, the blade mass and moment of inertia are for one blade only. This differs from the usual teetering rotor convention and results in equations containing terms such as $2 \mathrm{I}_{\mathrm{b}}$ instead of the more common $\mathrm{I}_{b}$.

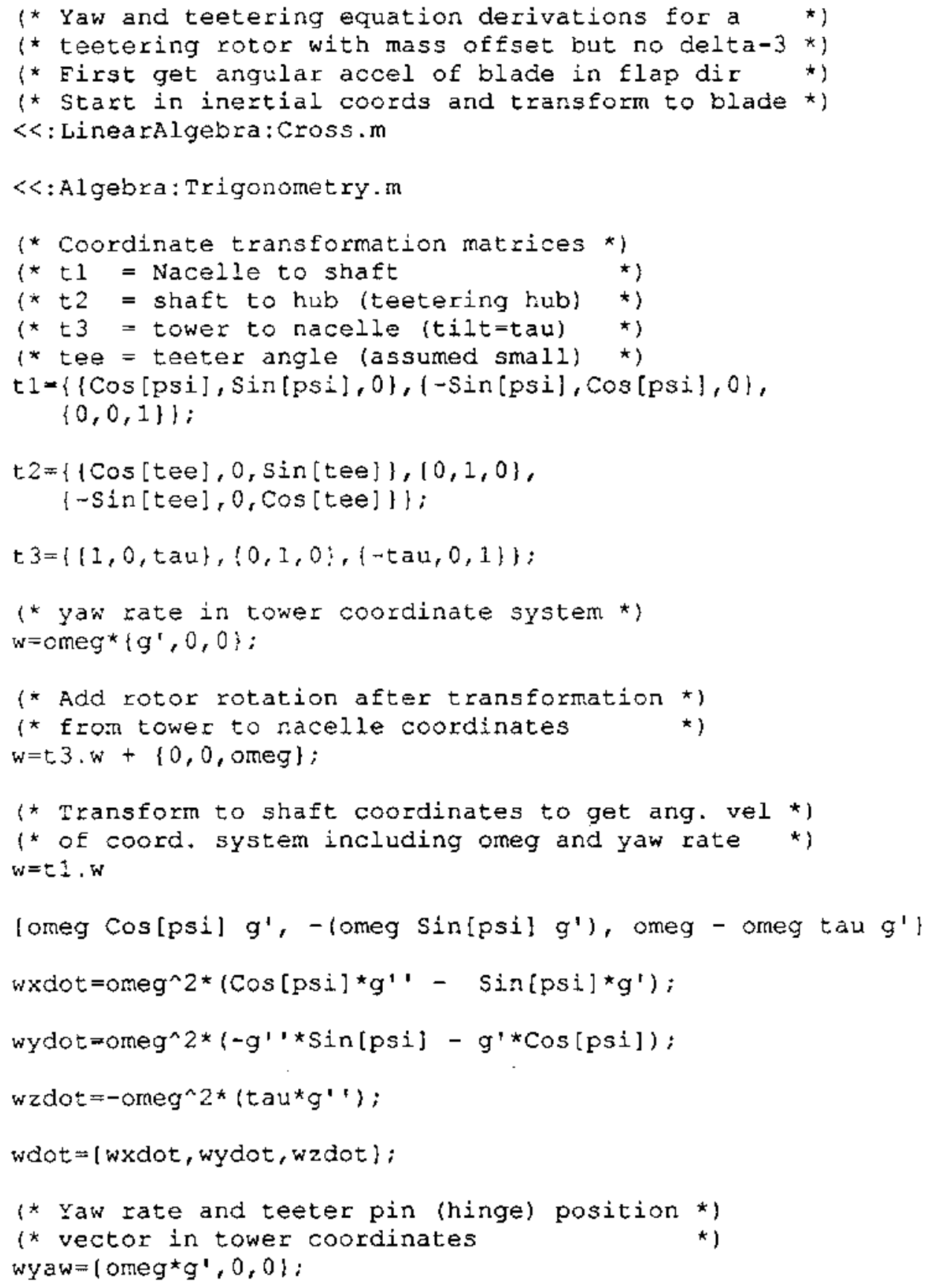




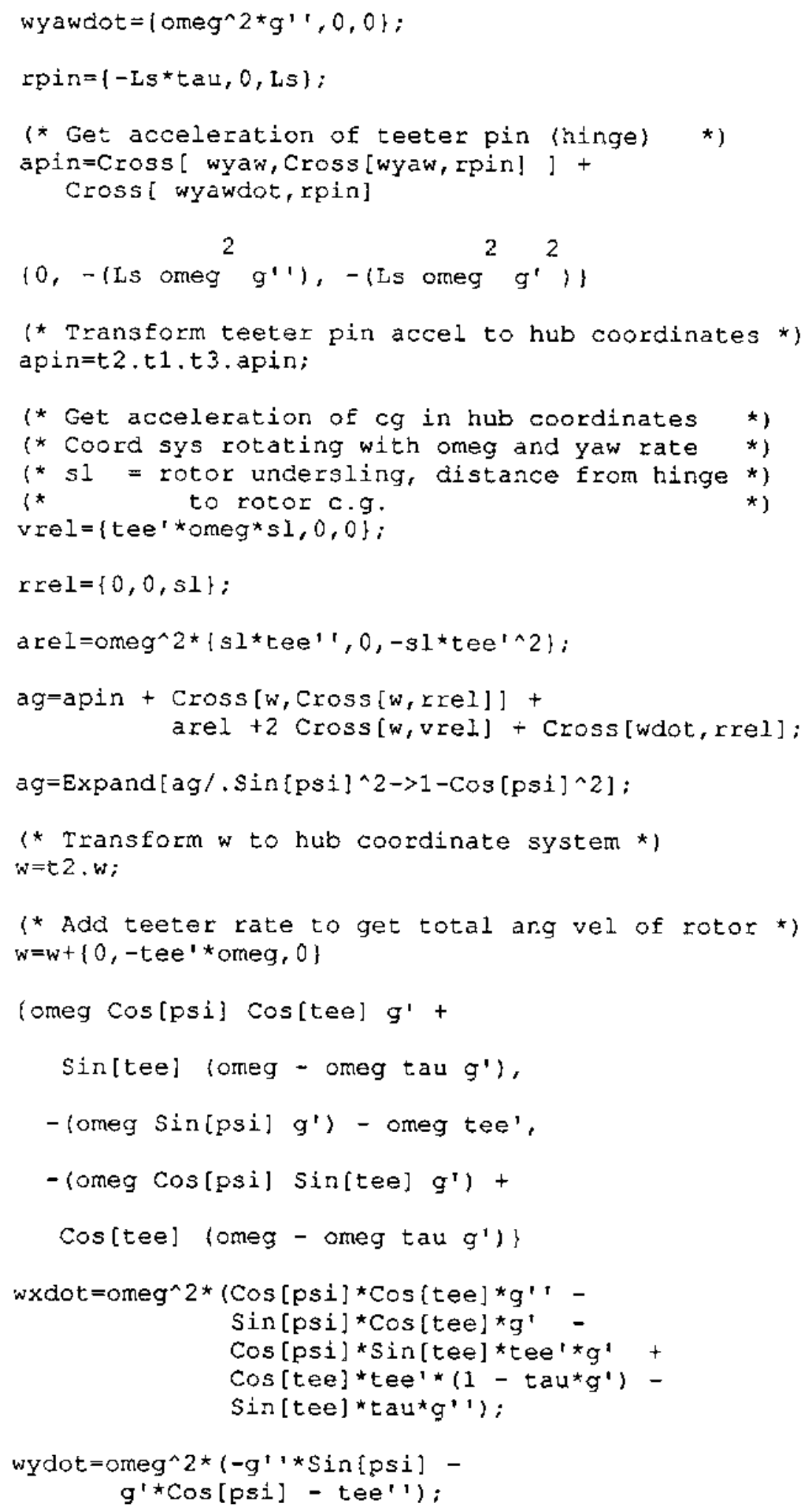




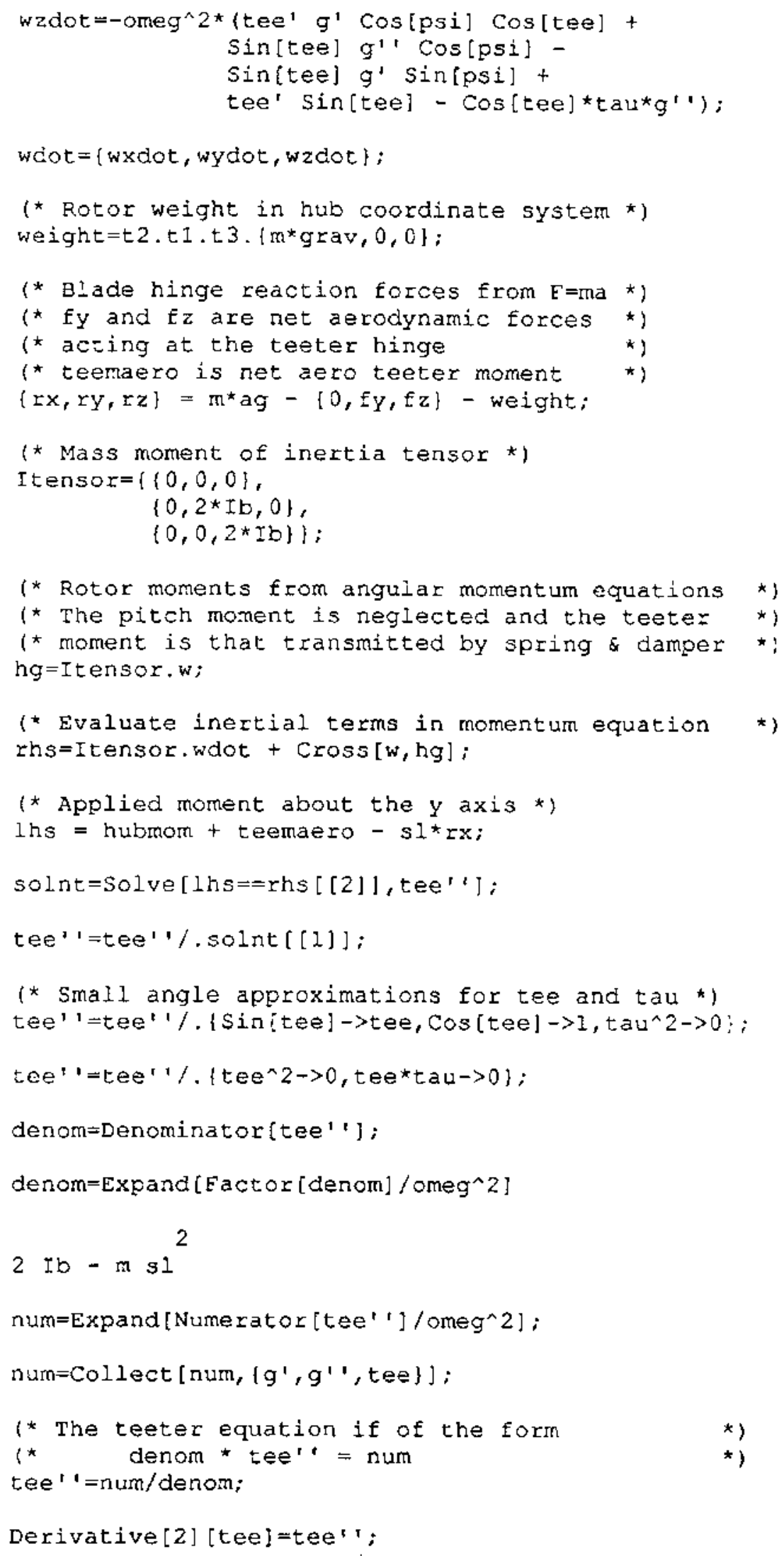




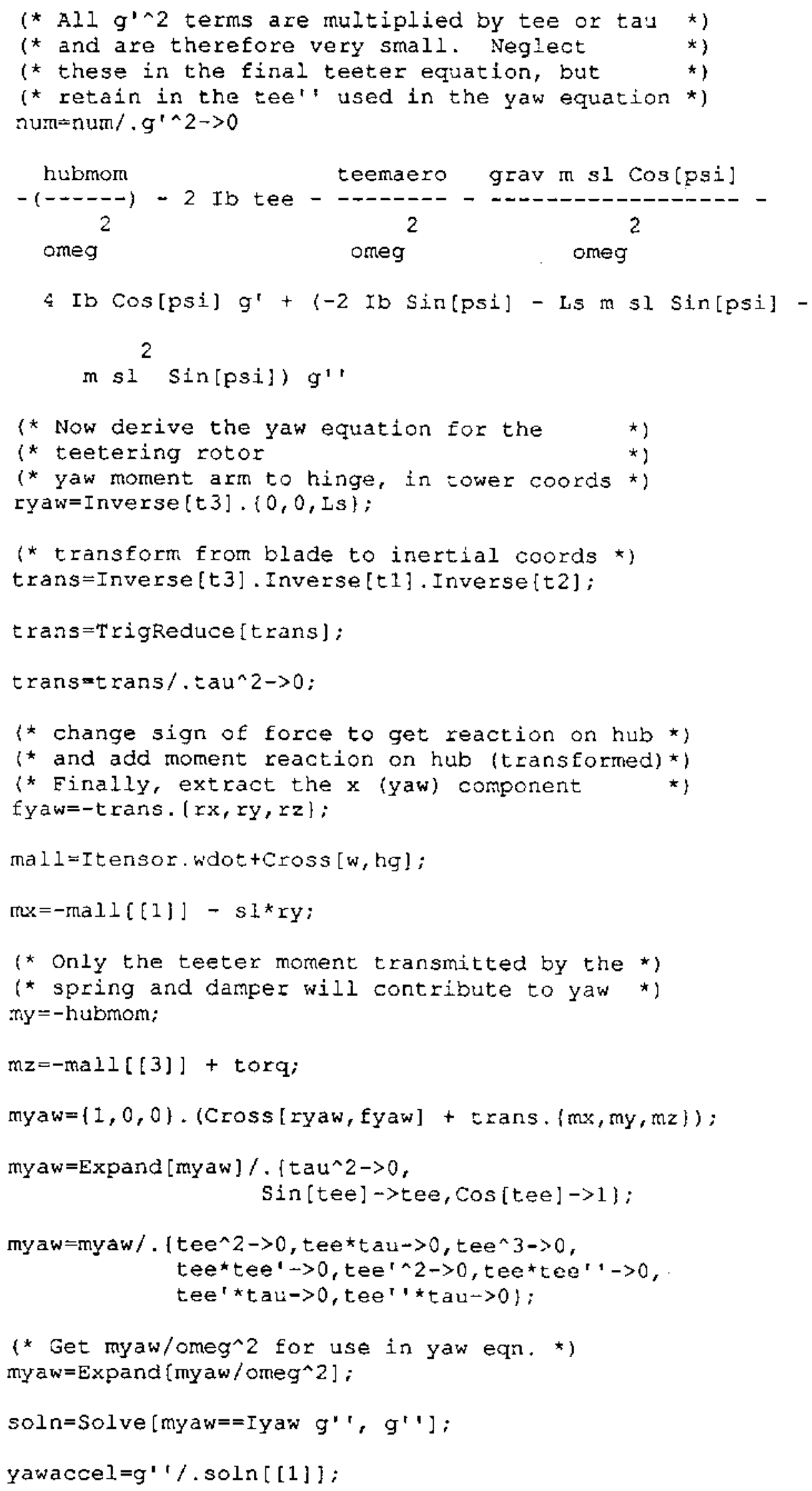




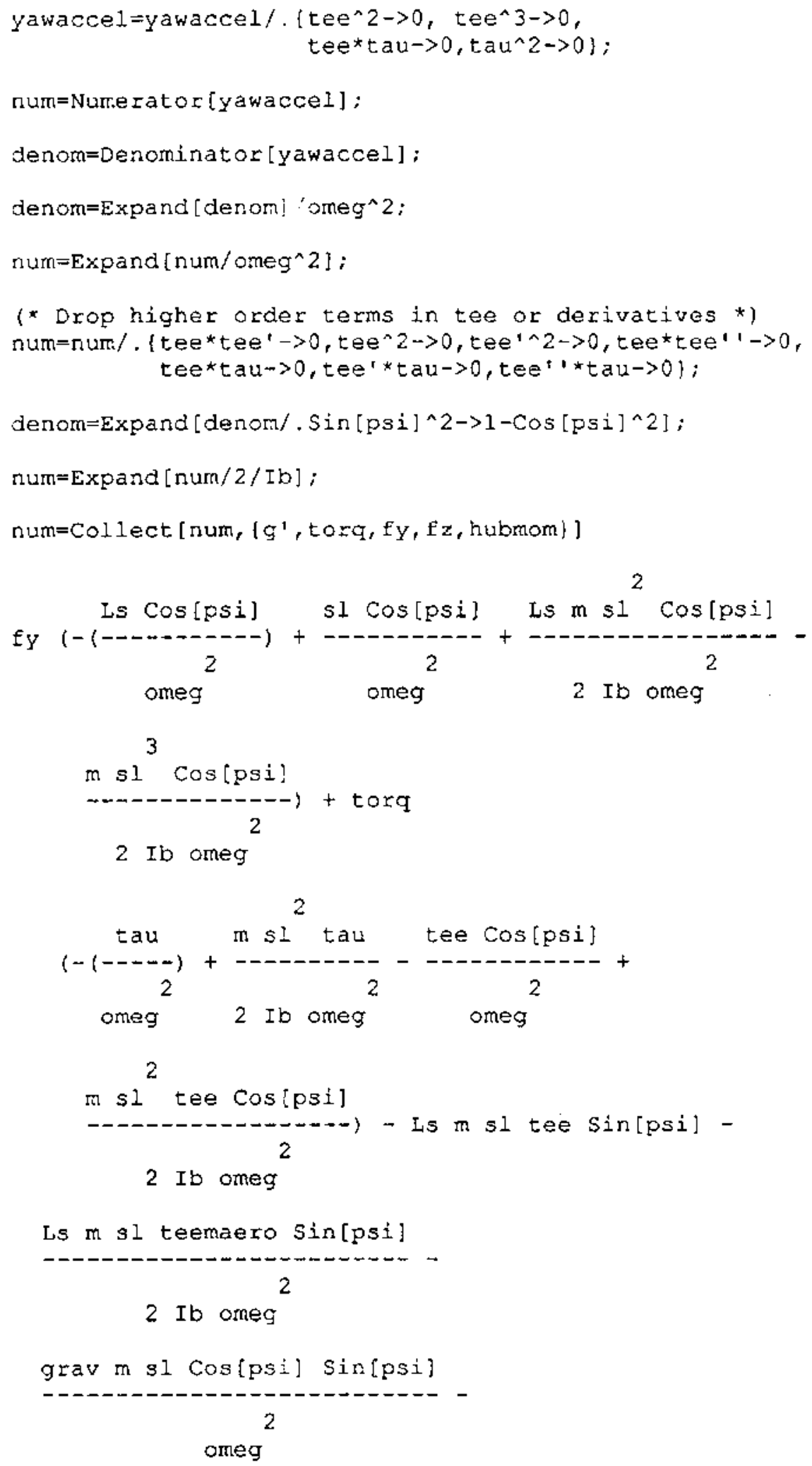




$$
\begin{gathered}
\text { Ls grav } \mathrm{m}^{2} \mathrm{si} \\
2 \\
2 \text { Ib omeg }
\end{gathered}
$$$$
\text { grav } m^{2} s^{3} \cos [p s i] \sin [p s i]
$$

$$
2
$$

2 Ib omeg
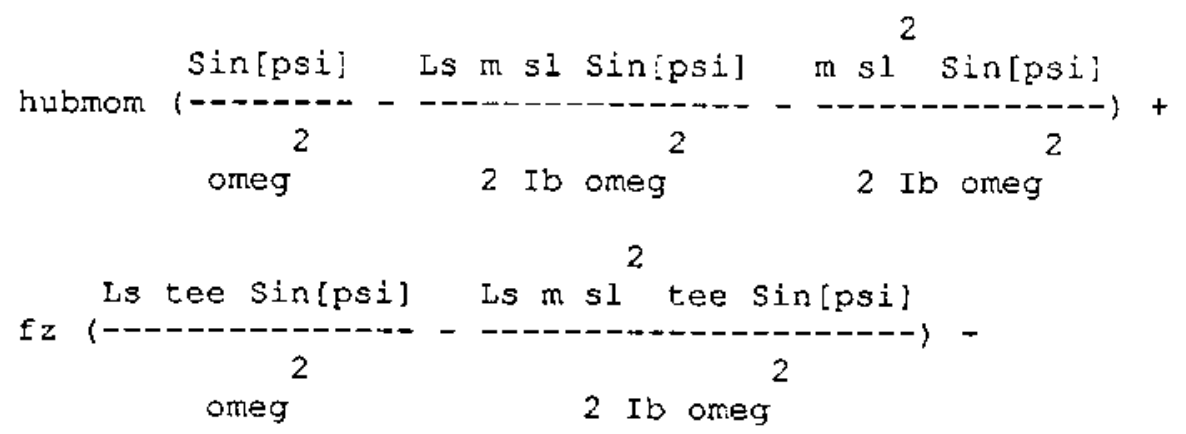

2 Ls $m$ sl Cos[psi] Sin[psi] $g^{\prime}+$

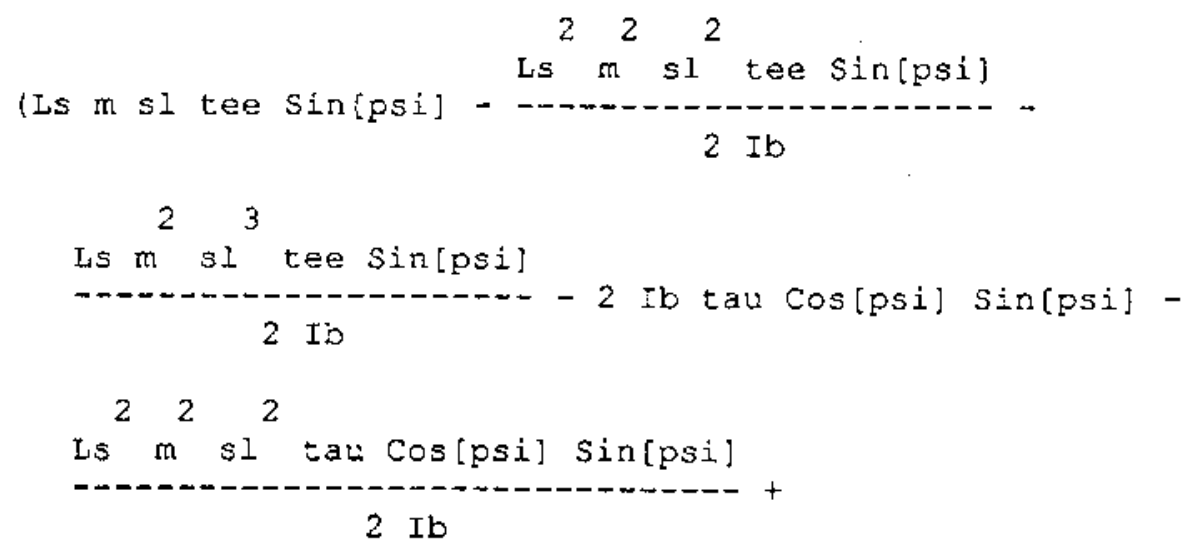




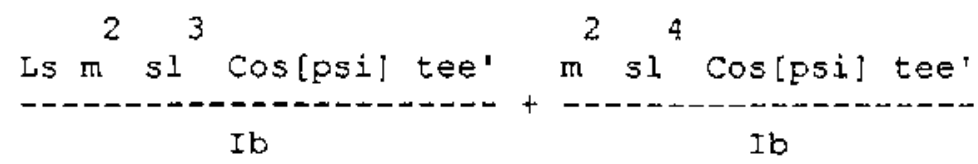

denom=denom $/ 2 / \mathrm{Ib}$;

Collect [denom, [Iyaw, Cos [psi]\}]

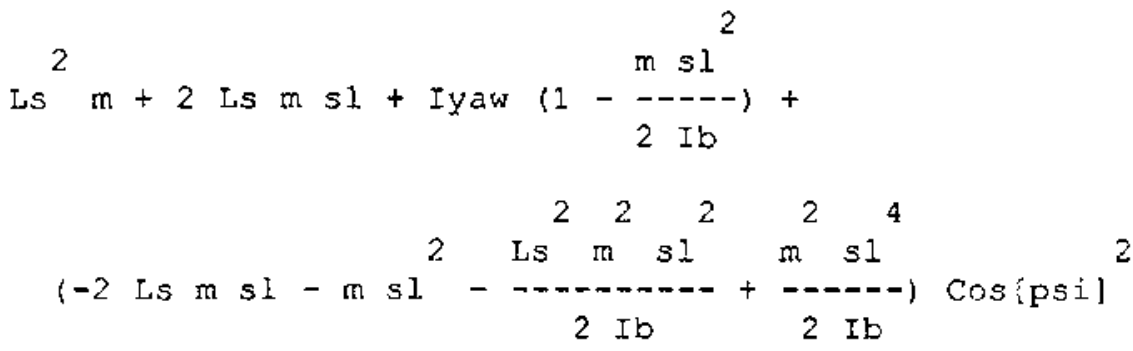




\begin{abstract}
Appendix B
Descriptions of the Combined Experiment, Mod-2 (Rigid Hub Wind-Tunnel Model) and ESI-80 Systems
\end{abstract}

This Appendix contains the wind turbine characteristics used in the YawDyn calculations presented in this report. The data were taken directly from a typical "YawDyn.opt" output file for each machine. The values were obtained from personal communications with SERI personnel.

\title{
SERI Combined Experiment Rotor
}

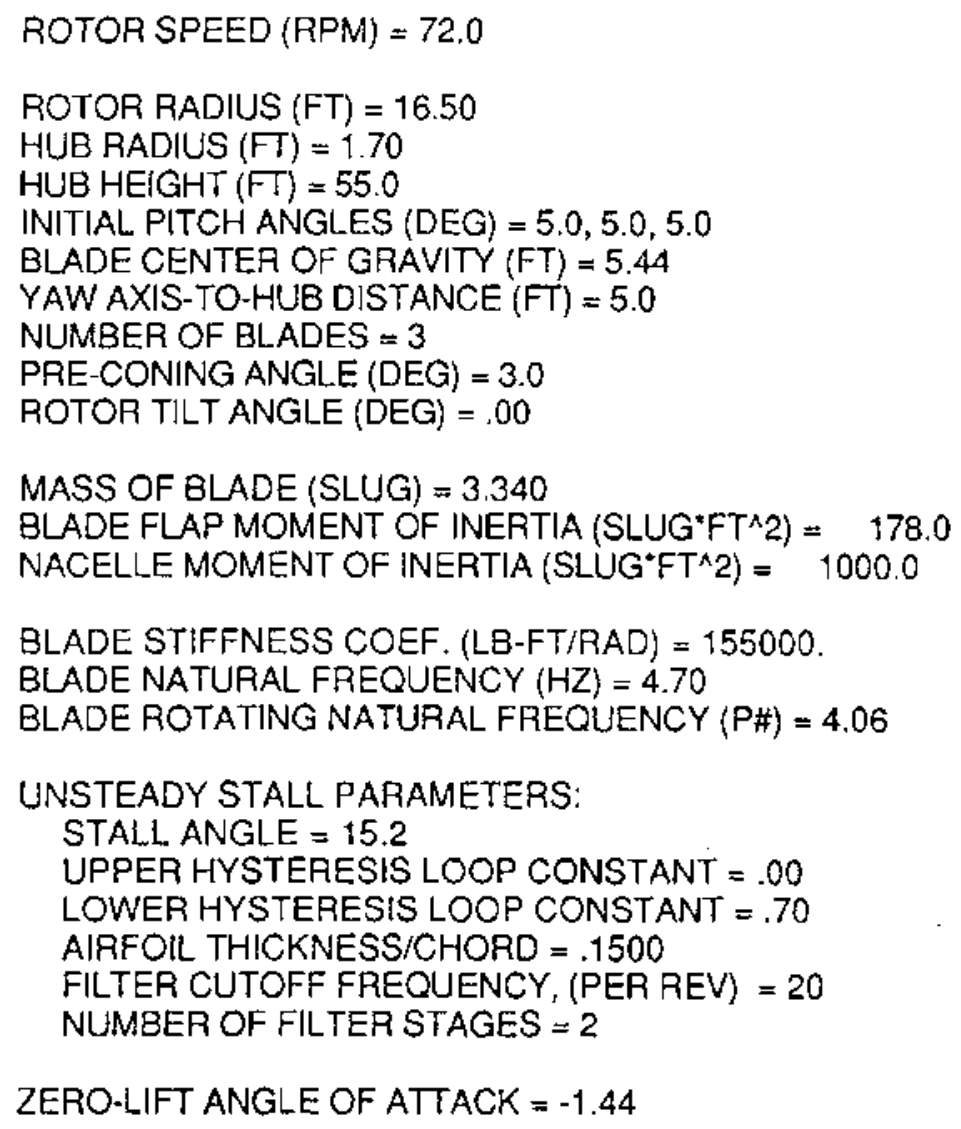


AIRFOIL CHARACTERISTICS:

$\begin{array}{cc}\text { ANGLE OF ATTACK(DEG) } & \text { LIFT COEF. } \\ -.01 & .1360 \\ 3.08 & .4410 \\ 6.16 & .7390 \\ 9.22 & .9210 \\ 12.22 & 1.0070 \\ 14.24 & 1.0290 \\ 15.24 & 1.0350 \\ 16.24 & 1.0070 \\ 18.20 & .8860 \\ 20.15 & .7840\end{array}$

AIRFOIL CHARACTERISTICS:

ANGLE OF ATTACK(DEG) DRAG COEF.

$\begin{array}{rr}. .01 & .0121 \\ 3.08 & .0133 \\ 6.16 & .0154 \\ 9.22 & .0373 \\ 12.22 & .0587 \\ 14.24 & .0891 \\ 15.24 & .1151 \\ 16.24 & .1548\end{array}$

Blade profile in $10 \%$ increments from root $(5 \%)$ to tip (95\%):

$\begin{array}{cc}\begin{array}{c}\text { TWIST ANGLE } \\ \text { (DEG) }\end{array} & \begin{array}{c}\text { CHORD } \\ (\mathrm{FT})\end{array} \\ 0.0 & \\ 0.0 & 1.50 \\ 0.0 & 1.50 \\ 0.0 & 1.50 \\ 0.0 & 1.50 \\ 0.0 & 1.50 \\ 0.0 & 1.50 \\ 0.0 & 1.50 \\ 0.0 & 1.50 \\ 0.0 & 1.50 \\ & 1.50\end{array}$

\section{ESI-80 Rotor}

ROTOR SPEED (RPM) $=60.0$

ROTOR RADIUS (FT) $=40.0$

HUB RADIUS $(F T)=3.00$

HUB HEIGHT $(F T)=80.0$

INITIAL PITCH ANGLES (DEG) $=0.0,0.0$

BLADE CENTER OF GRAVITY $(F T)=16.1$

YAW AXIS-TO-HUB DISTANCE $(F T)=6.79$ 
NUMBER OF BLADES $=2$

PRE.CONING ANGLE (DEG) $=7.0$

ROTOR TILT ANGLE (DEG) $=0.0$

MASS OF BLADE (SLUG) $=53.08$

BLADE FLAP MOMENT OF INERTIA (SLUG*FT^2) $=7350.0$

NACELLE MOMENT OF INERTIA (SLUG* ${ }^{*}{ }^{\wedge} 2$ ) $=500.0$

FREE TEETER ANGLE (DEG) $=6.00000$

FIRST TEETER STIFFNESS COEFF. (FT-LB/RAD) $=2.000000 \mathrm{E}+06$

SECOND TEETER STIFFNESS COEFF. $($ FT -LB/RAD $)=1.000000 E+08$

TEETER DAMPING COEF $(F T$-LB-S $)=20000.0$

ROTOR MASS OFFSET $(F T)=.180000$

UNSTEADY STALL PAFAMETERS:

STALL ANGLE $=14.0$

UPPER HYSTERESIS LOOP CONSTANT $=0.0$

LOWER HYSTERESIS LOOP CONSTANT $=.50$

AIRFOIL THICKNESS/CHORD $=.1500$

FILTER CUTOFF FREQUENCY, (PER REV) $=20.0$

NUMBER OF FILTER STAGES $=2$

ZERO-LIFT ANGLE OF ATTACK $=-4.00$

AIRFOIL CHARACTERISTICS:

ANGLE OF ATTACK(DEG) LIFT COEF.
$-4.0$
$-.0436$
.0
.4223
4.0
.8786
8.0
1.2557
12.0
1.4846
14.0
1.5217
15.0
1.5172
16.0
1.4957

AIRFOIL CHARACTERISTICS:

ANGLE OF ATTACK(DEG) DRAG COEF.

$\begin{array}{rr}-4.0 & .0125 \\ .0 & .0123 \\ 4.0 & .0147 \\ 8.0 & .0201 \\ 12.0 & .0290 \\ 14.0 & .0348 \\ 15.0 & .0380 \\ 16.0 & .0415 \\ 17.0 & .0453 \\ 18.0 & .0494\end{array}$


Blade profile in $10 \%$ increments from root $(5 \%)$ to tip (95\%):

$\begin{array}{cc}\begin{array}{c}\text { TWIST ANGLE } \\ \text { (DEG) }\end{array} & \begin{array}{c}\text { CHORD } \\ (F T)\end{array} \\ & \\ 1.0 & 1.00 \\ 2.0 & 2.20 \\ 1.9 & 2.50 \\ 1.8 & 2.90 \\ 1.5 & 2.70 \\ 1.2 & 2.50 \\ .8 & 2.20 \\ .1 & 2.00 \\ -.3 & 1.90 \\ -1.5 & 1.50\end{array}$

Mod-2, Bigid-Rotor Wind Tunnel Model

ROTOR SPEED (RPM) $=350.0$

ROTOR RADIUS $(F T)=7.5$

HUB RADIUS $(F T)=.00$

HUB HEIGHT (FT) $=10.0$

INITIAL PITCH ANGLES (DEG) $=.00 \& 0.00$

BLADE CENTER OF GRAVITY $(F T)=1.5$

YAW AXIS-TO-HUB DISTANCE $(F T)=-1.33$

NUMBER OF BLADES $=2.0$

PRE-CONING ANGLE (DEG) $=0.0$

ROTOR TILT ANGLE $(D E G)=0.0$

MASS OF BLADE (SLUG) $=.3285$

BLADE FLAP MOMENT OF INERTIA (SLUG*FT^2) = 2.2

NACELLE MOMENT OF INERTIA (SLUG*F'^2) = 2.0

BLADE STIFFNESS COEF. (LB-FT/RAD) $=22300.0$

BLADE NATURAL FREQUENCY $(H Z)=16.0602$

BLADE ROTATING NATURAL FREQUENCY $(\mathrm{P} \#)=2.929$

YAW STIFFNESS COEF. (FT-LB/RAD) $=0.0$

YAW AXIS FRICTION (FT-LB) $=0.0$

YAW AXIS DAMPING (FT-LB-SEC) $=0.0$

TOWER SHADOW COEFFICIENT $=0.0$

TOLERANCE FOR TRIM SOLUTION

CONVERGENCE TEST $=1.0 \mathrm{E}-02$

UNSTEADY STALL PARAMETERS:

STALL ANGLE $=18.0$

UPPER HYSTERESIS LOOP CONSTANT $=0.5$

LOWER HYSTERESIS LOOP CONSTANT $=0.5$

AIRFOIL THICKNESS $/$ CHORD $=0.15$

FILTER CUTOFF FREQUENCY, (PER REV) $=20.0$ 
NUMBER OF FILTER STAGES $=2$

ZERO-LIFT ANGLE OF ATTACK $=-1.3$

AIRFOIL CHARACTERISTICS:

$\begin{array}{cc}\text { ANGLE OF ATTACK(DEG) } & \text { LIFT COEF } \\ -10.26 & .7184 \\ -4.09 & -.2379 \\ .00 & .10 \\ 2.10 & .26 \\ 8.27 & .73 \\ 12.39 & 1.0570 \\ 16.43 & 1.1750 \\ 18.45 & 1.2165 \\ 20.45 & 1.2173 \\ 22.44 & 1.1938 \\ 24.42 & 1.1506 \\ 26.41 & 1.1164 \\ 30.24 & .660 \\ 35.27 & .7470 \\ 40.29 & .7861 \\ 45.29 & .8013 \\ 50.29 & .7790 \\ 60.00 & .70 \\ 70.00 & .55 \\ 80.00 & .37 \\ 90.00 & .10 \\ 100.00 & -.17 \\ 110.00 & -.40 \\ 120.00 & -.60 \\ 130.00 & . .80 \\ 140.00 & -.90 \\ 150.00 & -.80 \\ 160.00 & .60 \\ 170.00 & .80 \\ 180.00 & .00 \\ & \end{array}$

AIRFOIL CHARACTERISTICS:

$\begin{array}{cc}\text { ANGLE OF ATTACK(DEG) } & \text { DRAG COEF. } \\ -10.26 & .0368 \\ -4.09 & .0180 \\ .00 & .0149 \\ 2.10 & .0166 \\ 8.27 & .0310 \\ 12.39 & .0535 \\ 16.43 & .0981 \\ 18.45 & .1218 \\ 20.45 & .1522 \\ 22.44 & .2209 \\ 24.42 & .2209 \\ 26.41 & .2580 \\ 30.24 & .5060 \\ 35.27 & .6350 \\ 40.29 & .7540 \\ 45.29 & .8840\end{array}$




$\begin{array}{cc}50.29 & .9850 \\ 60.0 & 1.20 \\ 70.0 & 1.38 \\ 80.0 & 1.50 \\ 90.0 & 1.55 \\ 100.0 & 1.50 \\ 110.0 & 1.40 \\ 120.0 & 1.26 \\ 130.0 & 1.07 \\ 140.0 & .90 \\ 150.0 & .60 \\ 160.0 & .33 \\ 170.0 & .15 \\ 180.0 & .0\end{array}$

Blade profile in $10 \%$ increments from root $(5 \%)$ to tip (95\%):

$\begin{array}{cl}\begin{array}{c}\text { TWIST ANGLE } \\ \text { (DEG) }\end{array} & \begin{array}{l}\text { CHORD } \\ (\mathrm{FT})\end{array} \\ .0 & \\ .0 & .2250 \\ 4.50 & .2250 \\ 3.0 & .60 \\ 2.50 & .40 \\ 2.0 & .39 \\ .70 & .39 \\ .40 & .3850 \\ .30 & .37 \\ -2.50 & .36 \\ & .24\end{array}$




\title{
USER'S GUIDE \\ to the Yaw Dynamics Computer Program \\ YAWDYN
}

\author{
A.C. Hansen \\ $\mathrm{X}$. Cui \\ Mechanical Engineering Department \\ University of Utah \\ Salt Lake City, UT 84112 \\ User's Guide Date and Version \\ January, 1992 \\ Version 6 \\ Program date and version \\ YawDyn 5.4: 1/8/92
}

Prepared for the Solar Energy Research Institute under Subcontract No.

XL-6-05078-2 


\section{NOTICE}

This report was prepared as an account of work sponsored by the Solar Energy Research Institute, a Division of Midwest Research Institute, in support of its Contract No. DE-AC02-83-CH10093 with the United States Department of Energy. Neither the Solar Energy Research Institute, the United States Government, nor the United States Department of Energy, nor any of their employees, nor any of their contractors, subcontractors, or their employees, makes any warranty, express or implied, or assumes any legal liability or responsibility for the accuracy, completeness or usefulness of any information, apparatus, product or process disclosed, or represents that its use would not infringe privately owned rights. 


\section{TABLE OF CONTENTS}

Introduction....................................................

Disk Files Included with YawDyn $\ldots \ldots \ldots \ldots \ldots \ldots \ldots \ldots \ldots \ldots \ldots$

Nomenclature and Sign Conventions.............................5

Input Data File Description ,...................................

The YawDyn.Wnd Data File $\ldots \ldots \ldots \ldots \ldots \ldots \ldots \ldots \ldots \ldots \ldots \ldots \ldots$

Sample Input Data File ..................................... 19

User Operation at Run Time.................................. 20

Sample YawDyn.Opt File....................................22

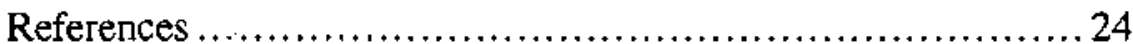




\author{
USER'S GUIDE \\ to the Yaw Dynamics Computer Program \\ YAWDYN \\ User's Guide Date and Version \\ January, 1992 \\ Version 6 \\ Program date and version \\ YawDyn 5.4: $1 / 8 / 92$
}

\title{
Introduction
}

This report is intended to provide information necessary to prepare inputs for the computer program YawDyn. YawDyn was developed with the support of the Solar Energy Research Institute Wind Research Branch for the analysis of the yaw motions and loads of a horizontal axis wind turbine with a rigid or teetering hub and two or three blades. In this document a detailed description of each of the program inputs and operating instructions will be given. A sample input file and output file are provided for testing the program operation. There is no discussion of the underlying theory or limitations of the models. That discussion is available in annual reports and joumal articles [see list of references]. In early reports on this program references were made to three independent programs, YAWDYN, SDOF, and UFLAP. The functions of all three programs are now combined into the single program which is the subject of this document, YawDyn.

This version of the User's Guide is current as of the date and version shown above. It is applicable to versions of the programs with the dates given on the cover page. Since the software development is continuing, and significant changes are continually being made to the programs, the reader should be certain the guide is appropriate to the program version that will be used.

\section{Disk Files Included with YawDyn}

Two files contain the source code for the YawDyn program. They are the main body of the program "YAWDYN.FOR" and a single Include file "YAWDYN.INC". The primary data input file is called "YAWDYN.IPT". If desired, a second input file called "YAWDYN.WND" can be read by the program. This file contains time-varying wind data (details are provided below). Up to three output files are created by YawDyn. The "YAWDYN.OPT" file is intended for printing a record of all the input conditions and a sample of the calculated results. File "YAWDYN.PLT" is tabular data intended for plotting results of the simulation with a variety of commercially available graphics packages. Finally, "YHARMON.IPT" is an optional output file intended for use with the program "YHARMON". YHarmon calculates the harmonic content (Fourier series) of the yaw and flap moments which are output by YawDyn.

\section{Nomenclature and Sign Conventions}

The analysis is directed toward a wind turbine with the general configuration shown in Figures 1 and 2 or 3 . The rotor can have 2 or 3 blades and the hub is rigid or teetering. The blade flap degree of freedom is modeled using an equivalent hinge and spring arrangement as shown in Figure 2 if the hub is rigid (not teetering). The teetering hub 
configuration is shown in Figure 3. Effects of undersling and the damping and stiffness characteristics of the teeter stop are included in the teetering model. The model assumes that all blades are identical in all respects except that each blade pitch angle is specified independently.

The definitions of yaw angle $(\gamma)$ and wind direction $(\delta)$ are shown in Figure 1 . (Note this is an unconventional definition of wind direction.) The yaw angle is the angle the rotor makes with the coordinate system, not with the instantaneous wind vector. Thus the yaw error (or difference between the compass rotor direction and wind direction) is $\gamma+\delta$. However, it is most common to use the program with the wind direction $\delta=0$. Then the yaw angle and the yaw error are the same. A positive vertical wind shear causes an increase in wind speed with height above ground. A positive horizontal wind shear causes an increase in wind speed with increasing coordinate y. See Figure 4.

The rotor can be downwind of the tower (positive $\mathrm{L}_{\mathrm{S}}$ in Figure 1 or FORTRAN variable $S L$ ) or it can be upwind (negative $L_{S}$ ). The blade hinge of the rigid hub can be of set an arbitrary distance from the axis of rotation. The location of the hinge defines both the structural hinge axis and the beginning of the aerodynamic surface of the blade. When a teetering rotor is modeled, the hub radius must be zero to place the teeter axis on the rotor axis of rotation.

The rotation of the rotor must be clockwise when viewed looking in the downwind direction. If the rotor to be analyzed actually tums in the counterclockwise direction, the user must be careful interpreting the sign conventions. It is best to consider the position of the blade when it is advancing into the region of increased relative wind speed (due to yaw angle or wind shear) and adjust the signs of the yaw angle and wind shears to be appropriate to this condition.

An example may clarify this potentially confusing topic. In the example, consider a downwind rotor which spins counterclockwise when viewed from a position upwind of the machine. In this case the rotor angular velocity vector (using the right hand rule) is directed from the hub toward the yaw axis and the rotation is opposite that used in the program. Consider also that the wind speed is higher on the left side of the rotor than on the right (when looking downwind). This situation is sketched in the views labeled "actual situation" in Figure 5. It is not possible to run the program with a negative (counterclockwise) rotor $\mathrm{rpm}$, so other signs must be adjusted. With yaw and horizontal wind shear the blade will be advancing into the wind when the blade is vertical upwards $\left(\psi=180^{\circ}\right)$ and the yaw angle is negative. If the rotor spin were clockwise the advancing blade would be at $\psi=180^{\circ}$ when the yaw angle is positive, and the horizontal shear is negative. Thus the change in the sense of rotation requires a change in the sign of the yaw angle and the horizontal wind shear to achieve the same conditions for the blade. This is depicted in the views labeled "model equivalent" in Figure 5. To summarize, the actual situation in Figure 5 has counterclockwise rotor rotation, a negative yaw angle and positive wind shear. This is modeled with clockwise rotation, positive yaw, and negative horizontal shear. The goal at all times is to keep the orientation of the advancing blade correct. 


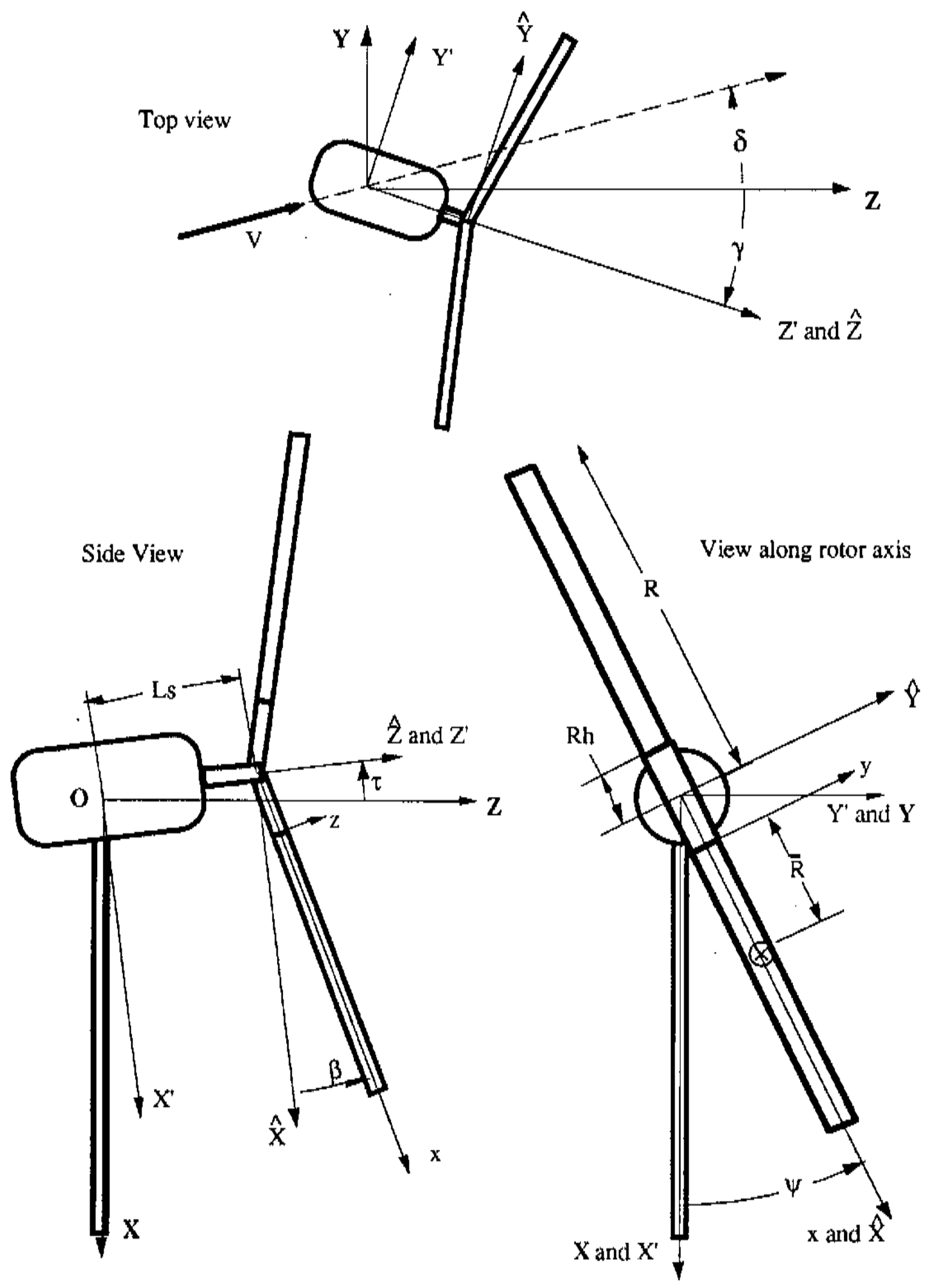

Figure 1. View of the HAWT defining selected terms and coordinates systems. The bold $\mathbf{X}, \mathbf{Y}, \mathbf{Z}$ axes are fixed in space while the $X^{\prime}, Y^{\prime}, Z^{\prime}$ axes are attached to the nacelle and yaw with the machine. The $\hat{X}, \hat{Y}, \hat{Z}$ system rotates with the shaft of the rotor and the $x, y, z$ system is attached to the blade, with Blade $\# 1$ in the direction of the positive $x$-axis. 


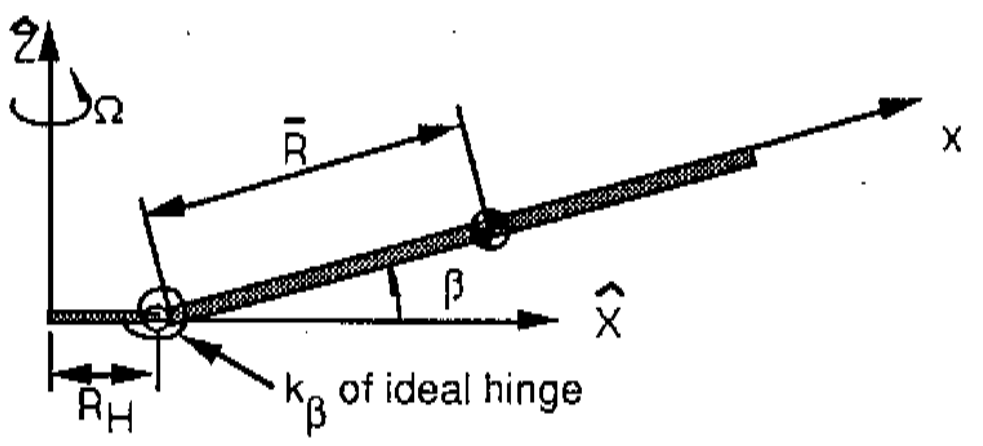

Figure 2. The equivalent hinge-spring model for the blade flap degree of freedom.

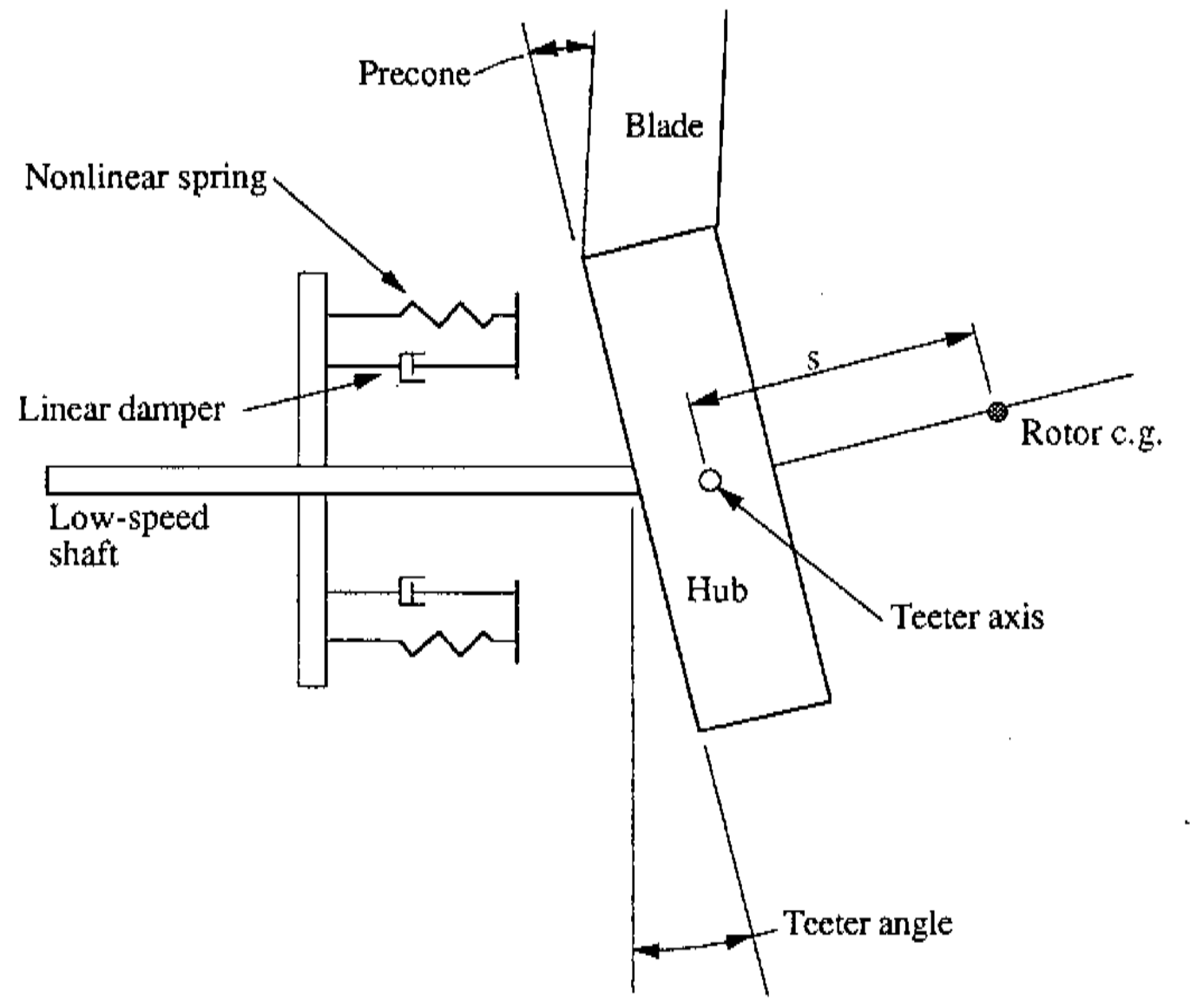

Figure 3. The configuration of the teetering hub model. The spring and damper are only active when the teeter deflection exceeds the angle TEE1. 

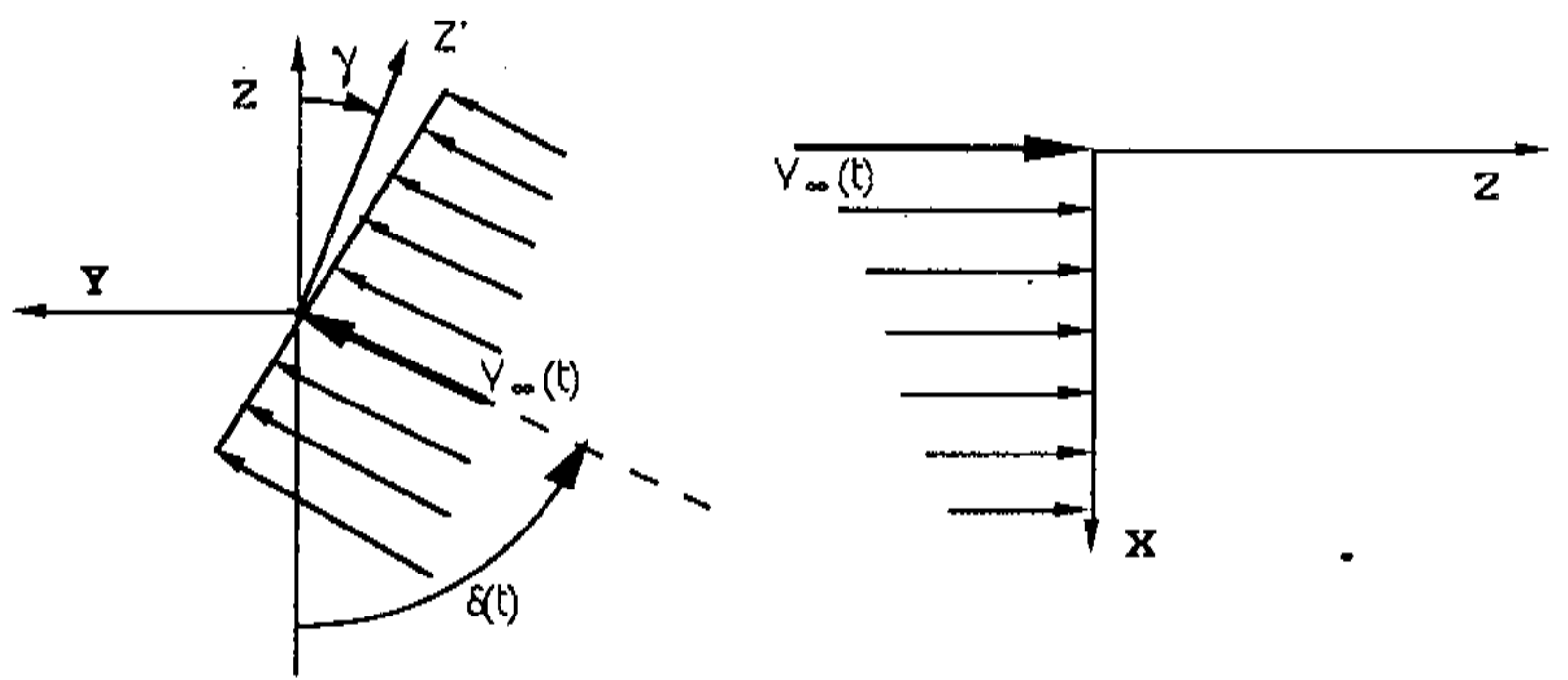

Figure 4. Wind shear models. Horizontal shear in left sketch, Vertical shear in right sketch. Note the wind direction $(\delta)$ and yaw angle $(\gamma)$ are both defined with respect to the $\mathbf{Z}$ axis. 
Actual Situation

View looking downwind

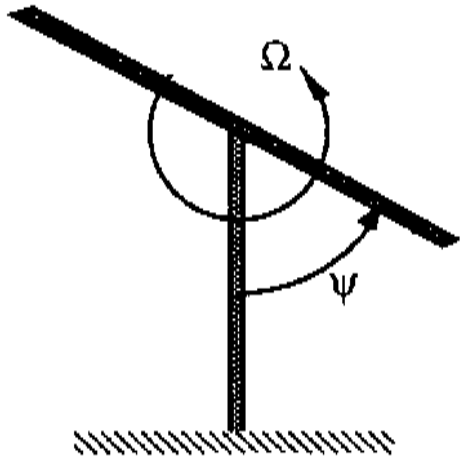

Top View

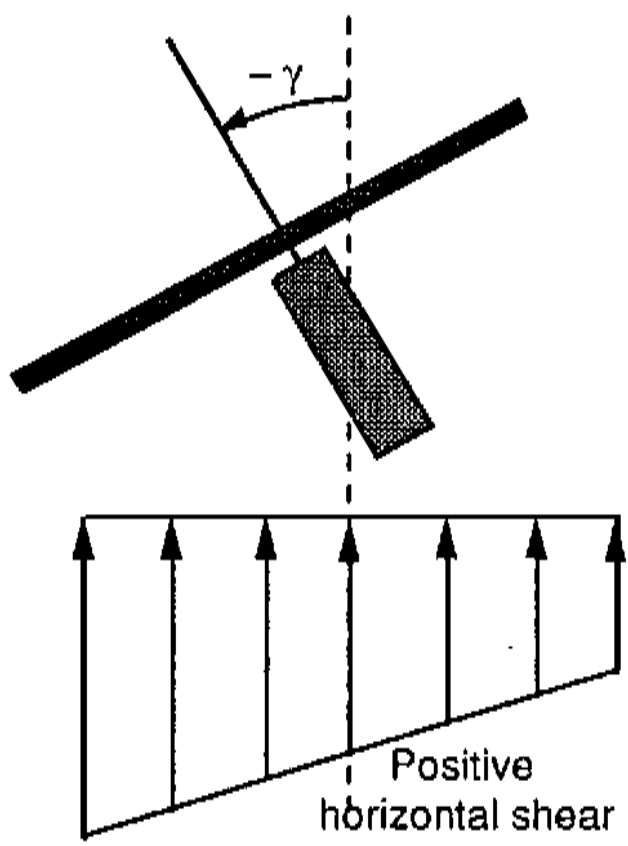

Model Equivalent View looking downwind

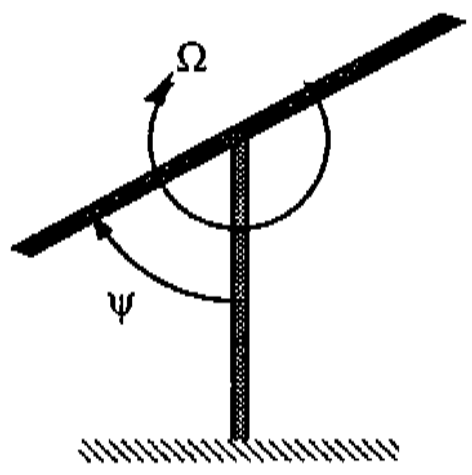

Top View

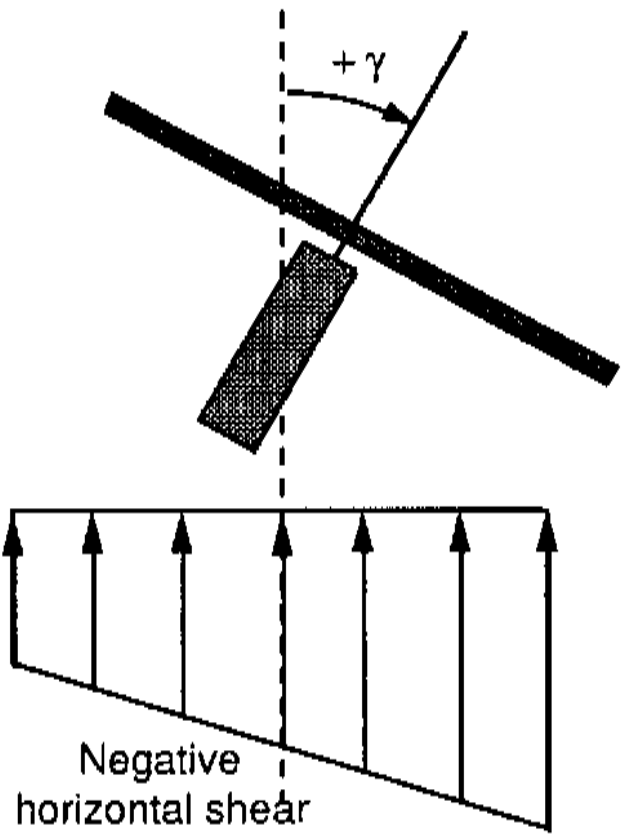

Figure 5. Views of example configuration with horizontal wind shear. Left half shows the actual configuration while the right side shows how that configuration can be modeled in YawDyn.

\section{Input Data File Description}

A sample input data file is given in Table 1. A text data file containing each of these items must be available in the directory or folder from which the program is run. The following is a description of each of the input variables. The formatting is list-directed (or free). There are no restrictions on the spacing of the values other than the order of the variables on a line, the order of the lines, and the presence (absence) of a decimal point in a floating point (integer) value. Values on one line should be separated by one or more spaces. Each 
line, except the first, can be terminated with a text string to identify that line. Each line must terminate with a return character. Each line must contain all of the variables specified for that line in the table below. Omission of a value, even if it is not used by the program in that particular run, will result in an execution error.

In the description that follows, the units for each parameter are listed for the program as it is used at the University of Utah. However, it is quite simple to change the program to run in SI units. The value of the gravitational constant, GRAV, must be changed in the program source code to GRAV $=9.81$. Then the units input to the program must all be consistent with the SI system (kg, m, sec, N, deg or rad as listed below, etc.).

The last three values on the second input line control the output of data to the CRT and data files. The volume of data makes it undesirable to print a record of all variables at all times and blade locations during a simulation. Instead, one particular revolution and one blade element are selected for tabulation of the most detailed information. Also, the time steps in the simulation are generally shorter than that needed for data output. The program will decimate the output if desired. The value IPRINT specifies the decimation factor.

\begin{tabular}{|c|c|c|c|c|}
\hline Line & Position & Name & Units & Description \\
\hline 1 & 1 & TITLE & -- & $\begin{array}{l}\text { Any character string ( }<80 \text { characters) to identify the } \\
\text { system being analyzed. This also serves as an aid to } \\
\text { identifying the contents of the data file. }\end{array}$ \\
\hline 2 & 1 & IYAWC & -- & $\begin{array}{l}\text { A flag to determine whether the simulation is for fixed } \\
\text { or free-yaw operation. If IYAWC }=0 \text {, the system is } \\
\text { rigidly fixed, if IYAWC }=1 \text {, then the system is } \\
\text { constrained by the yaw torsional spring. If the spring } \\
\text { stiffness (see line } 5 \text { ) is zero then the system is free- } \\
\text { yawing. }\end{array}$ \\
\hline 2 & 2 & MREV & -- & $\begin{array}{l}\text { Identifies the rotor revolution for which detailed data } \\
\text { will be sent to the YawDyn.opt and the YHarmon.ipt } \\
\text { files. }\end{array}$ \\
\hline 2 & 3 & NSEE & -- & $\begin{array}{l}\text { The blade element number for which detailed data will } \\
\text { be sent to the YawDyn.plt and YawDyn.opt files. The } \\
\text { value ranges from } 1 \text { for the inboard element to } 10 \text { for } \\
\text { the tip element. }\end{array}$ \\
\hline 2 & 4 & IPRINT & -- & $\begin{array}{l}\text { The decimation factor for data output. Typically, } \\
\text { printing every } 5 \text { th to } 10 \text { th time step will provide output } \\
\text { data with adequate resolution. }\end{array}$ \\
\hline 3 & 1 & FDOF & -- & $\begin{array}{l}\text { A flag to determine whether the flap degree of freedom } \\
\text { is included in the calculations. If FDOF=1, flap } \\
\text { motion and its effect on yaw loads are calculated. If } \\
\text { FDOF }=0 \text {, the flap angle is held constant at the precone } \\
\text { value and the flap rate is zero. (In the terminology of } \\
\text { early reports, program becomes SDOF when FDOF }=0 \\
\text { and it becomes the original YawDyn program when } \\
\text { FDOF=1.) }\end{array}$ \\
\hline
\end{tabular}


32 ITETER -.

33

IWND :--

34

DELTAT sec

35

IHARM --

41

YI

42

$\mathrm{BM}$
A flag to determine whether the rotor has a rigid or teetering hub. If ITETER $=0$ the hub is rigid, if ITETER $=1$ the hub is teetering. If the hub is teetering then the flap DOF becomes the teeter DOF and the blade is completely rigid. If ITETER $=1$ then FDOF must be 1 also.

A flag to identify the source of wind speed and direction data. If IWND $=0$, then the operating conditions such as wind speed, wind direction, pitch angle and wind shears are read from later lines in this data file. These constant values will be used for all calculations. If you wish to allow the winds or pitch angles to vary with time, then the desired values must be stored as a time series in file YawDyn.Wnd. If IWND $=1$, then the operating conditions are read from the data file YawDyn.Wnd. This makes it possible to run the program using actual values of operating conditions from test data or artificial time series of wind conditions.

This is the time interval for the sampled wind data in the file YawDyn.Wnd. The interval must be greater than the time step used in the integration (see variable 'SECTOR' below). During program execution the file is read as simulated time increases. The values of operating conditions at time $t_{1}$ are used for simulated time $t_{1}<t_{1}<t_{1}+$ DELTAT. Thus DELTAT should be kept small to avoid large step changes in the winds. A value of DELTAT must be given, whether YawDyn.Wnd is used or not. But if IOPT $=0$, the value of DELTAT is ignored, thus any value can be used. See the text in a later section of this document for more details on the YawDyn.Wnd file.

A flag which determines whether the output file YHarmon.ipt is created. Enter a value of 1 if you want the file created, 0 if you do not. The Yharmon.ipt file is used by another program, YHARMON.FOR, to calculate the harmonic content of the yaw moment and flap moment during one selected revolution of the rotor (the MREVth revolution).

slug- $\mathrm{ft}^{2}$ Mass moment of inertia of the main frame, nacelle and hub about the yaw axis. YI represents the total moment of inertia of all the yawing mass except the blades.

slugs The mass of one blade. Even when the rotor has a teetering hub the mass of just one blade should be entered in this location. 
BLINER slug-ft ${ }^{2}$ The blade mass moment of inertia about the flap axis (through the hinge point). If the rotor is teetering then BLINER represents one-half the moment of inertia of the entire rotor (including hub and any concentrated masses) about the teeter axis.

$5 \quad 1 \quad$ FS

$\mathrm{ft}-\mathrm{lb} / \mathrm{rad}$ The torsional spring constant of the equivalent flapping hinge spring at the blade root. This value is named $\mathrm{k}_{\beta}$ in the reports and literature and in Figure 2 above. When FDOF $=0$ or when ITETER $=1$, this value is not used (but it still must be present in the input file).

YAWSTF ft-lb/rad The torsional spring constant of the yaw drive or yaw brake system. This variable can be used to represent an equivalent stiffness of the yaw control system and the tower. The value must always be present in the data file, but is only used when "free yaw" is simulated. If the actual system stiffness is very high, then the system should be run in "fixed yaw".

This line is included only if the system has a teetering rotor. If the hub is rigid, skip to line 6.

5a 1 TEE1 deg

For a teetering rotor only, the teeter angle at which the first contact with the teeter "stop" is made. No mechanical teeter moment is applied at the hub if the absolute value of the teeter angle is less than TEE1. For teeter angles greater than TEE1, a nonlinear spring and a linear damper are active. See Figure 3 for a sketch of the teetering hub configuration.

5a $2 \quad$ SPRNG1 ft-lb

The first (linear) coefficient in the quadratic equation which describes the teeter spring or "stop". The moment applied by the teeter spring is given as $M=\operatorname{SPRNG} 1 \cdot \delta+\mathrm{SPRNG} 2 \cdot \delta^{2}$

Where $\delta$ is the spring deflection in radians and the sign is chosen appropriately for the direction of deflection.

5a 3 SPRNG2 $\mathrm{ft}$-lb The second coefficient in the equation which describes the teeter spring. SPRNG1 and SPRNG2 determine the shape of the parabolic spring which represents the teeter stop.

5a 4 TDAMP ft-lb-sec The coefficient of the linear teeter damping. The damper is active for all teeter angles greater than TEE1. The teeter moment (ft-lbs) due to mechanical damping on the teeter axis is TDAMP multiplied by the teeter rate in radians/sec

5a 5 SLING ft

The offset of the center of gravity of the rotor from the teeter axis. SLING is positive if the $\mathrm{c} . \mathrm{g}$. of the rotor is 
downwind of the teeter axis. Shown as "s" in Figure 3 .
61
$\mathbf{R}$
$\mathrm{ft}$
The rotor radius.
62
RB
$\mathrm{ft}$
63
RH
$\mathrm{ft}$
The distance along the blade from the hinge axis to the blade center of gravity.
This value affects the dynamic and aerodynamic analysis. It is the distance from the axis of rotation to the hinge axis (the hub offset). It also represents the distance to the first airfoil section on the biade. The aerodynamic analysis is only performed for blade elements outboard of the hub offset. To maintain the correct flap velocities, RH must be zero for the teetering rotor.

$\begin{array}{llll}6 & 4 & \mathrm{HH} & \mathrm{ft} \\ 6 & 5 & \mathrm{~B} & -- \\ 6 & 6 & \mathrm{PC} & \mathrm{deg} \\ 7 & 1 & \mathrm{VB} & \mathrm{ft} / \mathrm{sec} \\ 7 & 2 & \mathrm{VX} & \mathrm{ft} / \mathrm{s}\end{array}$

7

3

RPM

rpm

7

4

HSHR --
Hub height of the rotor above the ground.

Number of blades, $B \geq 2$, except the teetering rotor must have $B=2$.

Blade precone angle.

Horizontal wind speed at the hub (see IWND above for conditions when this value is used by the program).

Vertical component of the wind speed. (The $\mathbf{X}$ component, thus a positive value is a wind blowing down toward the ground.) This value is assumed constant in time and uniform over the rotor disc in the present analysis. Normaily the average value in flat terrain is zero, but in complex terrain the vertical wind can be very significant.

Rotor rotation speed.

A measure of the horizontal wind shear across the rotor disc. The value is typically -1 . $<$ HSHR $<+1$. and represents the wind speed at the $3 / 4$ radius on one side of the rotor, minus the wind at the $3 / 4$ radius on the opposite side of the rotor, divided by the hub wind speed. That is,

HSHR $=\frac{\left.\text { (Wind speed at } y=+\frac{3}{4} R\right)-\left(\text { Wind speed at } y=-\frac{3}{4} R \text { ) }\right.}{\text { Wind Speed at hub }(y=0)}$

A linear variation of wind speed across the disc is used for shear in the horizontal direction.

A measure of the vertical wind shear across the rotor disc. The value can assume two meanings, depending upon the value of ISHR (see next item). If linear shear is requested, then VSHR is defined in a manner identical to HSHR (except, of course, it applies to 
variations in the vertical direction). If power-law shear is requested, the value of VSHR is the exponent in the power law relationship. Typical values would then be 0.1 to 0.2 .

$7 \quad 6 \quad$ ISHR $\quad--$

A flag to indicate the type of vertical wind shear used in the calculations. If $I S H R=1$, then linear vertical shear is used. If ISHR=2, then power-law vertical shear is used. The horizontal wind shear is always linear shear.

$77 \quad$ VELDEF -

A measure of the strength of the velocity deficit in the wake of the tower (tower shadow). The value is the amplitude of the fractional decrease in hub wind speed at the center of the tower shadow. Typical values are 0.05 to 0.2 .

8 SL 1 f $\quad$ The distance from the yaw axis to the center of the hub (the vertex of the rotor cone). A positive value is used for a downwind rotor, a negative value for an upwind rotor.

ft-lb-sec The linear yaw damping coefficient. The yaw moment (ft-lbs) due to mechanical damping on the yaw axis is AV multiplied by the yaw rate in radians/sec.

$\begin{array}{llll}8 & 3 & \mathrm{AF} & \mathrm{ft}-\mathrm{lb}\end{array}$

The sliding friction moment. A constant yaw moment due to friction. Note this is not a friction coefficient.

$8 \quad 4 \quad$ TILT $\quad \mathrm{deg}$

The tilt angle of the rotor axis of rotation. The sign convention is not consistent with the coordinate system. That is, positive tilt is a rotation about the negative $\mathrm{Y}^{*}$-axis.

9 1-B PITCH deg The pitch angles of each blade. The values need not be the same for all blades, but a value must be entered for each blade.

$101 \quad \mathrm{Q}(3) \quad \operatorname{deg}$

Initial yaw angle for the solution. When a yaw drive stiffness is specified and the program is run for "free yaw", this angle also specifies the undeflected position of the torsion spring.

$102 \mathrm{Q}(4) \quad \mathrm{deg} / \mathrm{sec}$ Initial yaw rate for the solution.

$11 \quad 1-2 \mathrm{~B} \quad \mathrm{QP}$ array ${ }^{\circ} \& \%$ Initial flap angle and flap rate for each blade. (For a teetering rotor, only the values for blade \#1 must be input.) The first value is the flap angle for blade \#1. The second value is the flap rate for blade $\# 1$, and so on. These values are important to the efficient convergence to a rotor trim solution. It is suggested that when a rotor is analyzed for the first time, the flap angles should all equal the precone angle and the flap rates should all be zero. This will resulk in a slow but 

121
$N$
$--$
131
SECTOR --
132
TOLER deg
Number of rotor revolutions which will be calculated in the solution. This value determines the total run time of the simulation according to the relation Total Time $=\mathrm{N}^{*} 60 / \mathrm{RPM}$.

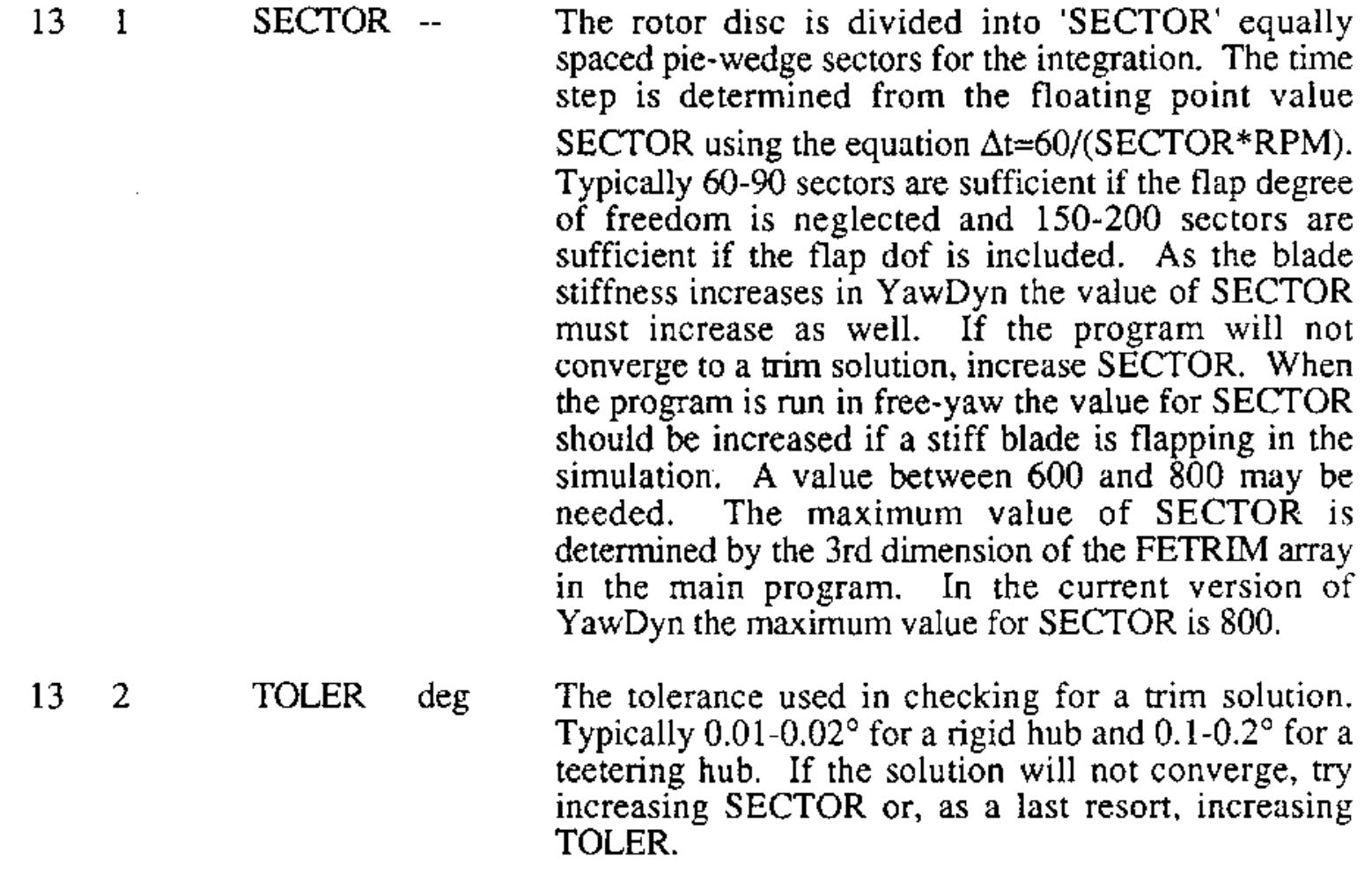

accurate convergence to a trim solution. When the trim solution is found, the values of flap and flap rate are output to the CRT. These values can be used in subsequent runs of the program to significantly reduce the time required to find the trim solution. When $F D O F=0$, these values are ignored by the program.

133 RHO slug/ $\mathrm{ft}^{3}$ Ambient air density.

141 ALPHAS deg This line of input pertains to the dynamic and static stall characteristics of the airfoil. The first parameter is the static stall angle of attack of the airfoil.

142 DSTLHI -.

The parameter in the Gormont dynamic stall model which determines the size of the upper hysteresis loop. The nominal value is 0.5 . If dynamic stall should not be included in the analysis, this parameter must equal zero.

143 DSTLLO

The parameter in the Gormont dynamic stall model which determines the size of the lower hysteresis loop. The nominal value is 0.5 . If dynamic stall should not be included in the analysis, this parameter must equal zero. 

144
THICK --
$\begin{array}{llll}15 & 1 & \text { CFILT } & \mathrm{P}\end{array}$
The thickness/chord ratio of the airfoil. This parameter is used only in the Gormont dynamic stall model. A typical value is 0.15 .
The use of dynamic stall requires digital filtering (smoothing) of the angle of attack time-history to achieve an accurate estimate of the rate-of-change of angle of attack. This parameter sets the cutoff frequency $(-3 \mathrm{~dB})$ of the lowpass filter. The value should be as large as is consistent with "smooth" angle of attack behavior (but less than SECTOR/5), A typical value is 30 (30 times the rotor rotation frequency). Low values will result in phase errors in the blade flap and subsequent errors in the yaw moments.

152 NFILT --

1611 NCL --

162 NCD --

163 ALPHAL deg

$\begin{array}{llll}171 & 1 & \text { AL } & \text { deg }\end{array}$

$\begin{array}{llll}17 & 2 & \mathrm{CL} & --\end{array}$

$\begin{array}{ccccc}-2 & 1 & \mathrm{AD} & \operatorname{deg} \\ -- & 2 & \mathrm{CD} & -.\end{array}$

${ }^{1}$ Line numbers 17 through $16+\mathrm{NCL}$

${ }^{2}$ Line numbers $17+\mathrm{NCL}$ through $16+\mathrm{NCL}+\mathrm{NCD}$
The number of stages of the digital filter. Allowed values are 1,2 , or 3 . Two stages typically are sufficient.

The number of points tabulated to specify the lift coefficient curve for the airfoil. The maximum value of NCL is 30 .

The number of points tabulated to specify the drag coefficient curve for the airfoil. The maximum value of NCD is 30 .

The zero-lift angle-of-attack of the airfoil.

The angle of attack for the first point in the lift coefficient table.

The lift coefficient corresponding to the angle of attack entered on this line. NCL lines such as this are entered to completely specify the lift coefficient vs. angle of attack curve. Flat-plate values of $\mathrm{CL}$ and $\mathrm{CD}$ are calculated by the program when the angle of attack is outside the range supplied in this table. Care must be taken to verify the complete CL-Alpha curve w

The angle of attack for the first point in the drag coefficient table.

The drag coefficient corresponding to the angle of attack entered on this line. NCD lines such as this are entered to completely specify the drag coefficient vs. angle of attack curve. 
The blade is described in terms of 10 equally spaced elements. The last ten lines of the input data file provide the twist and chord distribution for the ten elements. The first value of TWIST is the twist angle at the inner-most element $(r / R=0.05)$. The last line of the input file contains the twist and chord of the blade tip element $(\mathrm{r} / \mathrm{R}=0.95)$.

-2 CHORD $\mathrm{ft}$. The blade chord of the first (inner) blade element.

Integration of the equations of motion is accomplished using an explicit, forward stepping technique called the Adams-Bashforth method. There is no internal self-testing to determine if the step size (determined by the variable SECTOR) is small enough to give accurate results. Hence, the user must verify that SECTOR is large enough. The simplest way to do this is to run the same data set with increasing numbers of sectors until the results no longer change. Of course there is incentive to keep SECTOR as small as possible because the total run time of the simulation is proportional to the number of time steps. Generally, as the stiffness of the blade hinge increases the size of the time step must decrease to maintain accuracy.

When a free-yaw rotor with blade flapping is modeled, the system typically has a "stiff" set of equations. That is, there is a large difference between the high flap frequency and the low yaw motion frequency. YawDyn will be more stable when a large value of SECTOR (perhaps 800) is used for these free-yaw cases. This is particularly the case when the blade is quite stiff (say, for example 3 or $4 p$ ). These very stiff systems will tend to show numerical instability when the yaw motion is very slow. For example, when a rotor is released from rest with a yaw error, the solution will proceed smoothly as the rotor yaws to align with the wind. But after the rotor is aligned and the yaw rate becomes small, then numerical problems may appear. This should not be a problem as that phase of rotor operation can easily be handled by running a fixed-yaw simulation.

${ }^{2}$ Line numbers $17+\mathrm{NCL}+\mathrm{NCD}$ through $26+\mathrm{NCL}+\mathrm{NCD}$ (the last ten lines of the file) 


\section{The YawDyn.Wnd Data File}

If the parameter IWND in line 3 of the YawDyn.ipt file is equal to 1, the program will look for a tabular time series of operating conditions in file YawDyn. Wnd. (If IWND $=0$, then the file YawDyn. Wnd need not be present.) In the current version of the program the values of wind speed at the hub (VB), wind direction (DELTA), horizontal wind shear (HSHR), and vertical wind shear (VSHR) are entered in tabular form as a function of time (TDATA). Read statements which access this file are found in two locations in the program. The first is in the main program, and the second is in subroutine GETWND. Both statements are of the form READ $(11, *)$ TDATA, VB, DELTA, HSHR, VSHR. If desired, the list of variables read in can be shortened or extended to meet particular requirements. All that is required is to change these two READ statements. One parameter which might be added is blade pitch. If that is done one additional change may be required. Since the program retains pitch angles for each blade independently, the YawDyn. Wnd file must contain pitch data for each blade [i.e. $\operatorname{READ}\left(11,{ }^{*}\right)$ TDATA, VB, ... ( $P I T C H(I), I=1, N B)]$. However, if all pitch angles are the same, one value may be read and program lines must be added to equate the pitch of each blade to the data value. That is,

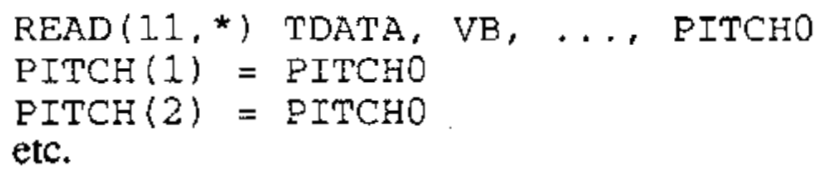

It can be seen from the READ statement that the tabular values can be separated by spaces in the YawDyn.Wnd file, all values read in for a particular time TDATA must be on one line, and each line must end with a return character. 
Table 1.

Sample Input Data File for the SERI Combined Experiment Wind Turbine

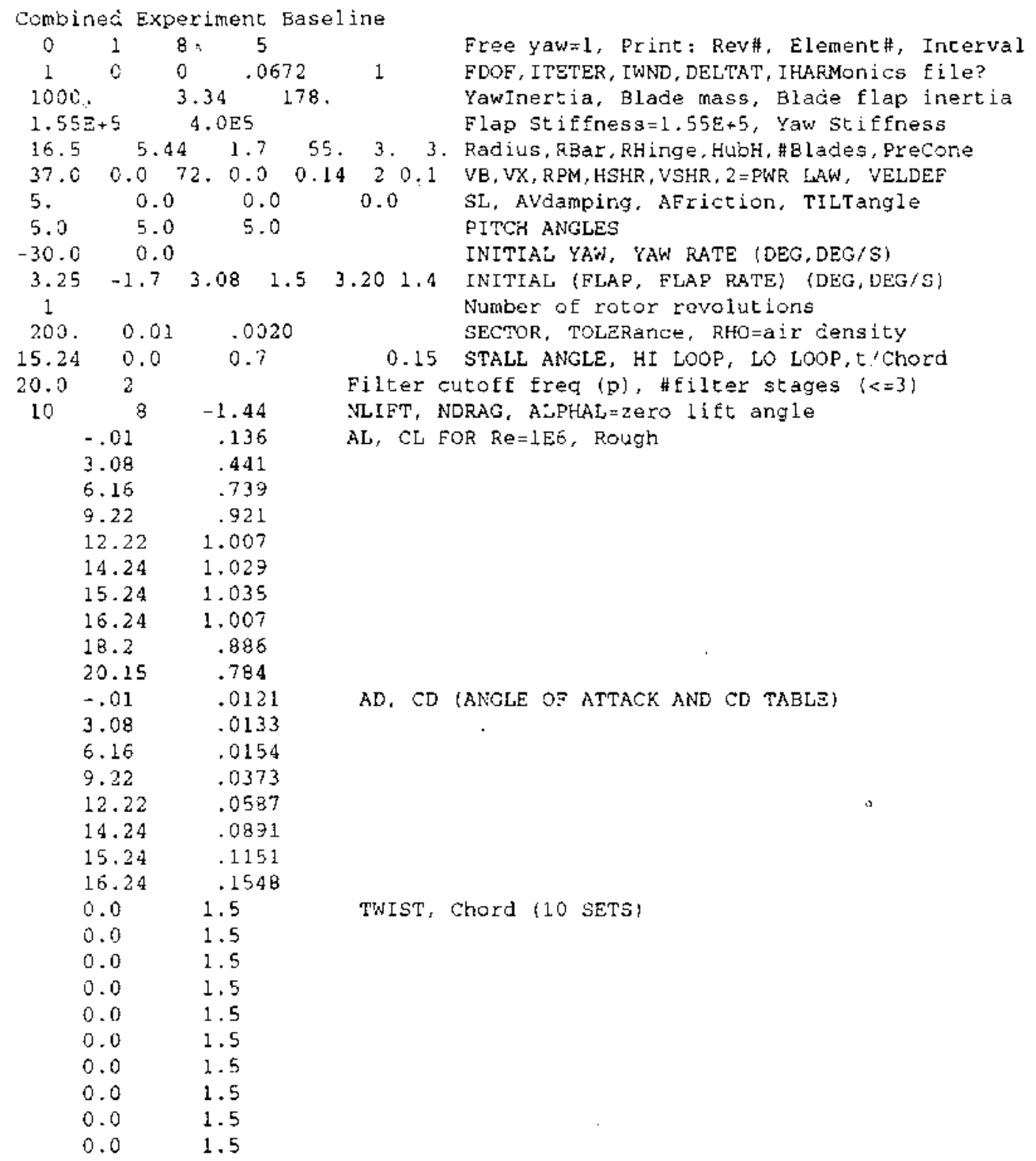




\section{USER OPERATION AT RUN TIME}

No user input is required after the program is run. The CRT will display information on the status of the calculations and a few statements about the run conditions so that the calculations can be interrupted if the desired conditions are not being run. The lines below are typical of what will be seen as the program executes. The courier font is used for information that will be sent to the CRT. Annotations are shown in the Helvetica font.

The search for the trim solution will continue until all "RMS ERROR" values are less than the "TOLER" value from the input file. If 30 trim revolutions are run before the solution converges to a trim condition the calculation will be aborted. If this occurs, use of different initial conditions, a larger value of SECTOR, or a larger tolerance on the trim criteria should be tried.

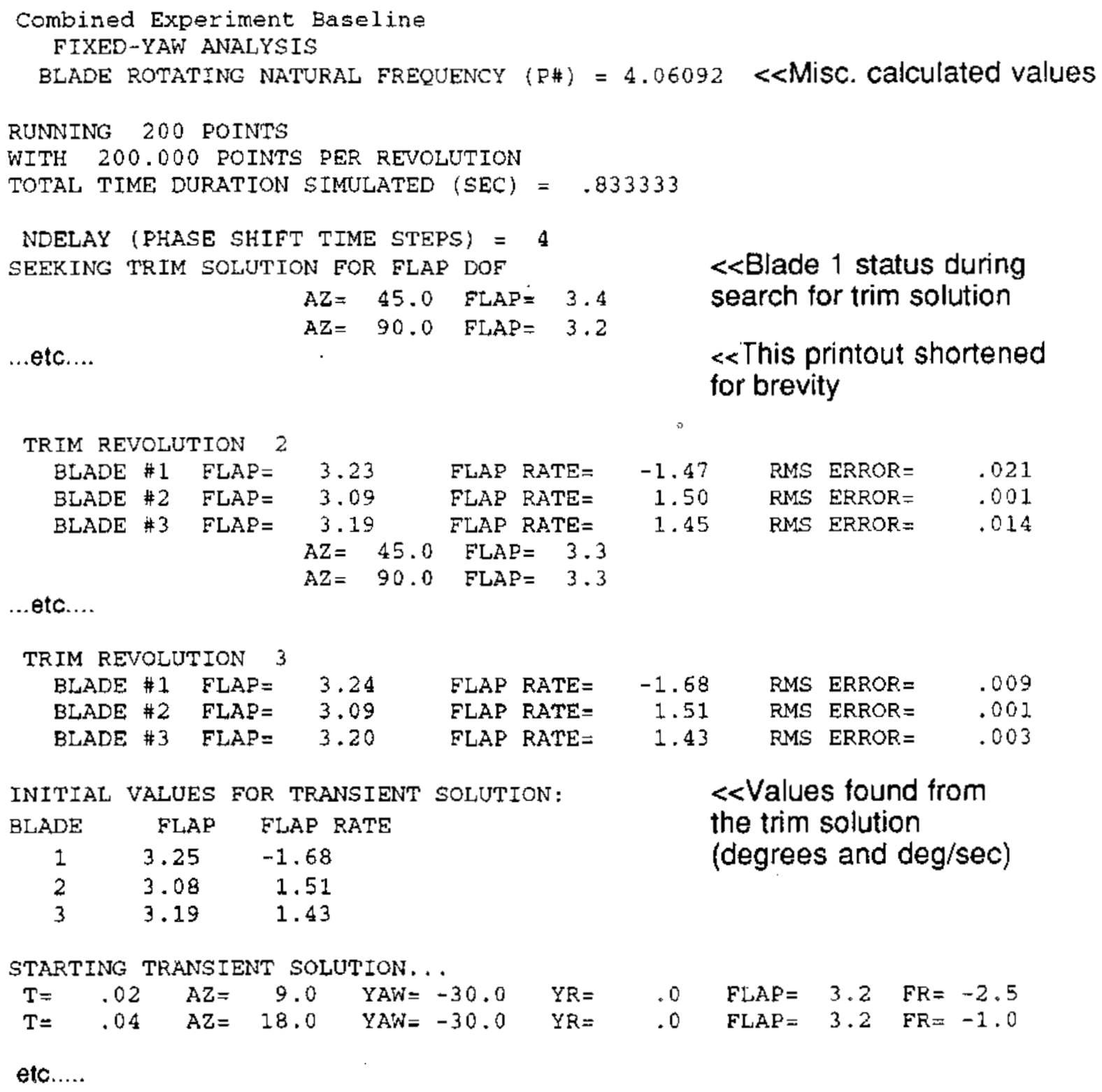


The output file YawDyn.opt is intended for printing a summary of the simulation conditions and some representative results. More detailed results are provided in the YawDyn.Plt file. In all files and displays the blade flap angle is the angle, $\beta$, which the blade makes with the plane of rotation. If a teetering rotor is being modeled, the teeter angle is the flap angle minus the precone angle.

The output file YawDyn.Plt is useful for plotting predictions as a function of time. The columns in the table are separated by tabs to allow the file to be read by many graphics software packages written for desktop computers. The first line of the file gives column headings, also separated by tabs. If your graphics package will not permit reading of the column headings in this way, delete the first (and only) line of text. 


\section{Sample YawDyn.Opt file from Program YawDyn \\ Using input file given in Table 1.}

\section{FILE YAWDYN.OPT:}

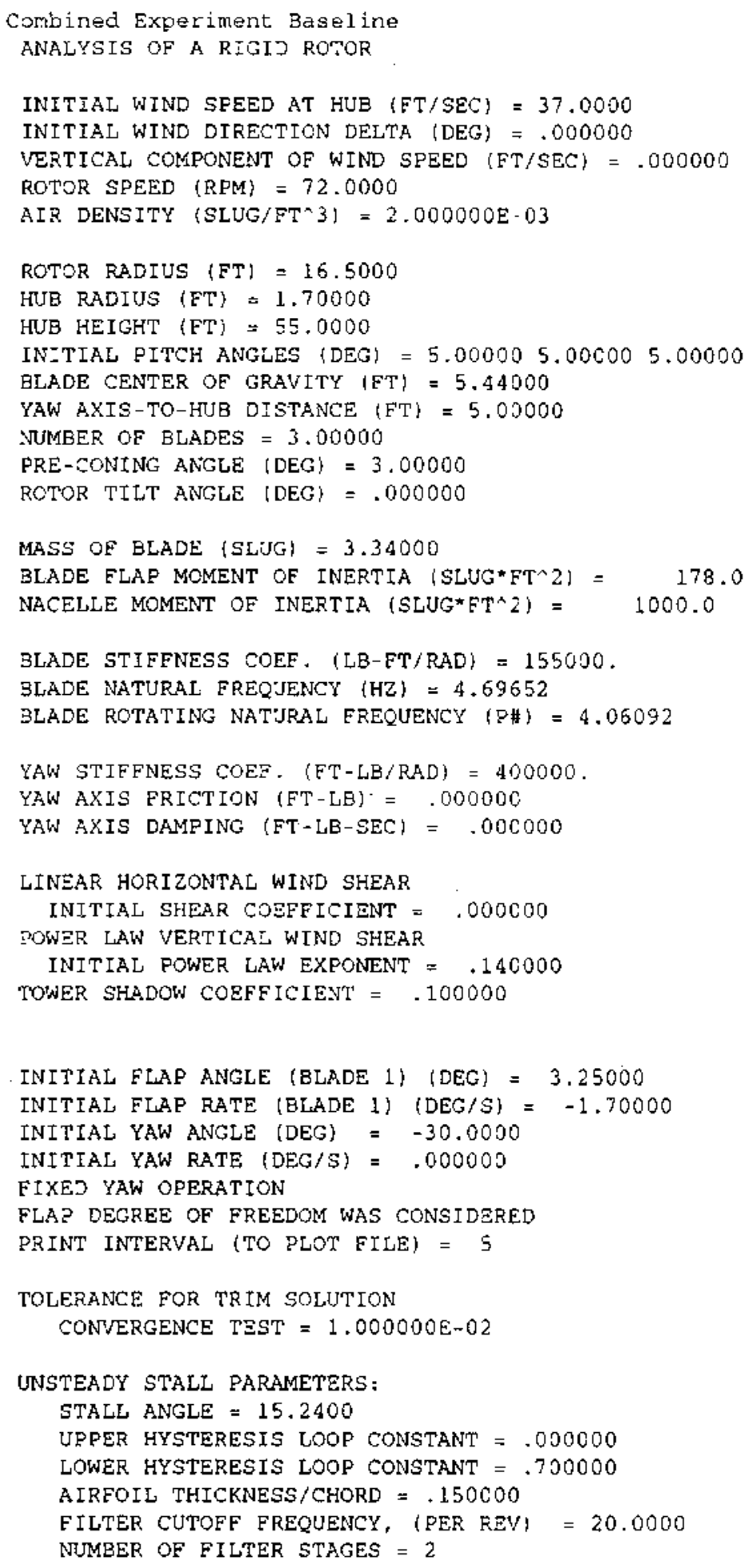




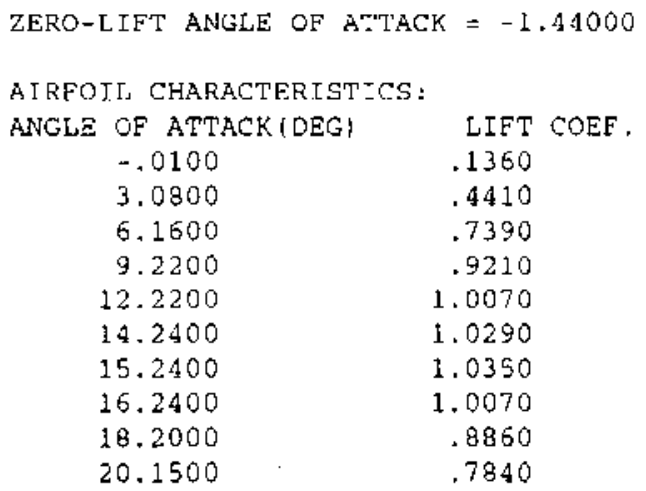

AIRFOIL CHARACTERISTICS:

$\begin{array}{cc}\text { ANGLE OF ATTACK (DEG) } & \text { DRAG } \\ -.0100 & .0121 \\ 3.0800 & .0133 \\ 6.1600 & .0154 \\ 9.2200 & .0373 \\ 12.2200 & .0587 \\ 14.2400 & .0891 \\ 15.2400 & .1151 \\ 16.2400 & .1548\end{array}$

$\begin{array}{cc}\begin{array}{c}\text { TNIST ANGLE } \\ \text { (DEG) }\end{array} & \begin{array}{c}\text { CHORD } \\ \text { (FT) }\end{array} \\ .0000 & 1.5000 \\ .0000 & 1.5000 \\ .0000 & 1.5000 \\ .0000 & 1.5000 \\ .0000 & 1.5000 \\ .0000 & 1.5000 \\ .0000 & 1.5000 \\ .0000 & 1.5000 \\ .0000 & 1.5000 \\ .0000 & 1.5000\end{array}$

FLAP MOMENT IS THE BIAADE DEFLECTION TIMES THE SPRING STIFFNESS. VAW MOMENT IS APPLIED AERODYNAMIC MOMENT

DATA FOR CYCLE NUMBER 1, BLADE ELEMENT 8

\begin{tabular}{|c|c|c|c|c|c|c|c|}
\hline PSI & YAW & YAWMOMENT & FLA PMOMENT & ALPHA & CL & $\mathrm{CD}$ & A \\
\hline $\mathrm{DEG}$ & DEG & $F T-L B$ & $\mathrm{FT}-\mathrm{EB}$ & $D E G$ & & & \\
\hline 1.8 & -30.0 & $-.2134 E+03$ & $0.6550 \mathrm{E}+03$ & 5.99 & .647 & .015 & .250 \\
\hline 3.6 & -30.0 & $-.1933 E+03$ & $0.6317 \mathrm{E}+03$ & 6.16 & .618 & .015 & .247 \\
\hline 5.4 & -30.0 &.$-: 7145+03$ & $0.6068 E+03$ & 6.42 & .603 & .017 & .241 \\
\hline 7.2 & -30.0 & $-.2477 \Xi+03$ & $0.5793 E+03$ & 6.73 & .602 & .019 & .235 \\
\hline 9.0 & -30.0 & $-.1231 \Xi+03$ & $0.5512 \mathrm{E}+03$ & 7.03 & .608 & .022 & .228 \\
\hline$=0.8$ & -30.0 & $-.9739 \varepsilon+02$ & $0.5227 \mathrm{E}+03$ & 7.31 & .631 & .024 & .222 \\
\hline$=2.6$ & -30.0 & $-.7220 \Omega+02$ & $0.4950 \mathrm{E}+\mathrm{C} 3$ & 7.52 & .673 & .025 & .217 \\
\hline$: 4.4$ & -30.0 & $-.5 i 45 E+02$ & $0.4702 E+03$ & 7.65 & .750 & .026 & .214 \\
\hline 16.2 & -30.0 & $-.4003 \Xi+02$ & $0.4485 E+03$ & 7.69 & .830 &.$C 2 \overrightarrow{6}$ & .211 \\
\hline 28.0 & -30.0 & $-.2092 \mathrm{E}+01$ & $0.4352 \mathrm{E}+\mathrm{C} 3$ & 7.73 & .832 & .027 & .208 \\
\hline 19.8 & -30.0 & $0.3718 \Omega+02$ & $0.4286 \mathrm{E}+03$ & 7.78 & .835 & .027 & .205 \\
\hline
\end{tabular}

(center portion of table deleted for brevity) 


$\begin{array}{llllllll}340.2 & -30.0 & 0.1387 \mathrm{E}+01 & 0.7720 \mathrm{E}+03 & 6.89 & .730 & .021 & .243 \\ 342.0 & -30.0 & -.5126 \mathrm{E}+02 & 0.7724 \mathrm{E}+03 & 6.90 & .738 & .021 & .242 \\ 343.8 & -30.0 & -.9991 \mathrm{E}+02 & 0.7705 \mathrm{E}+03 & 6.92 & .746 & .021 & .241 \\ 345.6 & -30.0 & -.1442 \mathrm{E}+03 & 0.7668 \mathrm{E}+03 & 6.93 & .756 & .021 & .240 \\ 347.4 & -30.0 & -.1809 \mathrm{E}+03 & 0.7616 \mathrm{E}+03 & 6.88 & .768 & .021 & .240 \\ 349.2 & -30.0 & -.2096 \mathrm{E}+03 & 0.7551 \mathrm{E}+03 & 6.74 & .774 & .020 & .242 \\ 351.0 & -30.0 & -.2297 \mathrm{E}+03 & 0.7466 \mathrm{E}+03 & 6.55 & .762 & .013 & .244 \\ 352.8 & -30.0 & -.2429 \mathrm{E}+03 & 0.7370 \mathrm{E}+03 & 6.34 & .750 & .017 & .248 \\ 354.6 & -30.0 & -.2522 \mathrm{E}+03 & 0.7249 \mathrm{E}+03 & 6.13 & .736 & .015 & .251 \\ 356.4 & -30.0 & -.2530 \mathrm{E}+03 & 0.7111 \mathrm{E}+03 & 5.97 & .721 & .015 & .253 \\ 358.2 & -30.0 & -.2472 \mathrm{E}+03 & 0.6949 \mathrm{E}-03 & 5.90 & .713 & .015 & .253\end{array}$




\section{References}

Cui, X. Hansen, A.C. and Siedschlag, N., 1988, "Yaw Dynamics of Horizontal Axis Wind Turbines: First Annual Report". SERI Technical Report SERI/STR-217-3309.

Hansen, A.C. and Cui, X., 1989, "Yaw Dynamics of Horizontal Axis Wind Turbines: Second Annual Report". SERI Technical Report SERI/STR-217-3476.

Hansen, A.C. and Cui, X, "Analyses and Observations of Wind Turbine Yaw Dynamics". ASME Journal of Solar Energy Engineering, Vol 111, November, 1989. Also presented at the ASME Energy Technology Conference, New Orleans, LA, January, 1988. 


\section{Listing of the YawDyn Program and Subroutines}

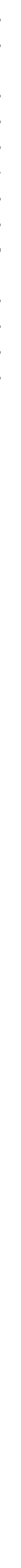

VERSICN $5.4,1 / 8 / 92$

YANDYN CALCLLATES YAW ANGLE, YAW RATE, YAW MOMENT FLAP ANGLE, ELAP RATE AND ELAF MONENT FOR EACH BLADE DE A 2 OR 3-BLADED HAWT. OPTIONS ARE AVAILABLE FOR OPERATING IN EIXED YAN OR WITHOUT FLAP CEGREE OF FREEDOM, A TEETERING ROTOR OPTION IS ALSO AVAILABLE. WHEN THE PEETERING ROTOR IS ANALYZED, THE YODEL HAS ONLY TWO DEGREES OE FREEDOM-MHE TEETER ANGLE AND THE YAW ANGLE. THAT IS, THE BLADE STIFFNESS AND EREOUENCY ARE NO LONGER CONSIDERED. BUT EROVISIONS ARE INCLUDEJ FOR SPRING AND DAMPER TEETER STOPS.

WHEN TEETERING MOT:ON IS CALCULATED, THE TERMS FLAP AND TEETER ARE EQUIVALENT FOR THE NUMBER 1 BLADE (EXCEPT THAT THE FLAP ANGLE AND THE TEETER ANGLE DIFEER BY THE CONSTANT PRECONE)

EILE YAWDYN. INC MUST BE PRESENT WHEN THIS PROGRAM IS COMPILED

FILE YAWDYN. IPT CONTAINS THE HAWT DATA AND INITIAL CONDITIONS.

FILE YAWDYN.OPT IS AN OUTPUI SUMMARY (ECHO OF INPUT DATA AND DETAZLS OF CALCULATIONS DURING ONE REVOLUTIONI.

FILE YAWDYN.PLT IS AN OUTPUT TABLE FOR THE ENTIRE RUN.

FILE YAWDYN.WND CONTAINS ACTUAL OFERATING PARAMETER OATA SUCH AS WIAD VECTOR, SEE SUBROUTINE GETWND FOR DETAILS

ALL I/O UNITS ARE FEET, SLUGS, SECONCS, POUNDS FORCE AND DEGREES. UNI'TS CAN BE CHANGED TO SI EQUIVALENIS BY CIANGING VALUE OF THE CONSTAN" NAMED 'GRAV' AND USING CONSISTENT UNITS

EILE YHARMON, IFT CAN BE CREATED CONTAINING YAW AND TLAP MOMENT RESULTS FOR ONE SELECTED KEVOLUTION OF THE ROTOR.

THIS FILE IS USED EY PROG!AM YLARMON.FOR TO CALCULATE GARMONIC CONTENT OF THE YAN AND FLAP LOADS.

WRIT:EN BY XUDONG CUI ANC CRAIG HANSEN,

UNIVERSITY OF UTAH, MECHANICAL ENGINEERING DEPARTMENT

REVISION RECORD (AFTER 5/90):

5/24/90 CHANGE ALL VELOCITIES TO NONDIMENSIONAL FORM $V /\left(R E V S^{\star} R\right), Q(2) / R E V S$ AND $Q(4) / R E V S$

$6 / 9 / 90$ SKEWED WAKE INFLOW MOLEL MODIFIED.

8/5/90 REMOVED PITCH AND ROLL MOMENTS FROM THE INE SOW MODEL

8/24/90 REMOVED BLADE PITCH AND EDGE MOMENTS OE INERTIA RE-ARRANGED INPUT FILE (VERSION 3.3)

10/10/90 RETURNED TO USING INCLUDE FILE FOR COMMON STMTS (3.4)

2/28/91 REMOVED MANY AERO SUBROUTINES TO YAWSUBS.F

3/01/91 V3.5, CHANGED RUNGE-KUTTA (RK) TO SECOND ORDER PRED.-CORRECTOR (PREDCOR) TO SIMPLIFY THE PROGRAM. NO CHANGE IN RESULTS.

3/16/91 INCORPORATED ALL ASPECTS OF THE 'YAWTEETER' CODE AND YAWDYN V3.5 INTO A SINGLE PROGRAM WITH THE NEW TEETER INPUT DATA REQUIRED WHEN TEETER USED $\{4.0\}$.

6/14/91 NEW YAW DOF EQN. IMPLEMENTED (PREVIOUS VERSIONS HAD ERROR WHICH AFFECTED COJPLED FREE YAW/FLAP VERSION ONLY) ALSO FIXED BLG IN CALL F2 (..,PSIA) \{THE MISSING A\} (5.0)

6/26/91 INCORPORATED SHAFT TILT INTO CORRECTED EQNS OF MOTION. INCORPORATED FULL FREE-YAW TEETER EQUATIONS AND MOVED INTERACTIVE INPUT TO YAWDYN. IPT FILE. $(5.21$

8/30/91 FIXED BUG IN YAW MOMENT OUTPUT (RIGID ROTOR NITH FLAP ONLY) CHANGED FM TO FMYM IN YAWM CALCULATION (SUB. AERO) MINOR COSMETIC CHANGES AND RECOMBINATION WITH ALL 
SUBROLTINES TO CREATE THE EINAL FORM OF YAWDYN. (5.3) 1/08/92 CIANGEJ FIAA MOMENT WHICH IS OUTPUT FOR TEETER ROTCR $(5.4)$

PARTIAL LIST OF VARIAELES:

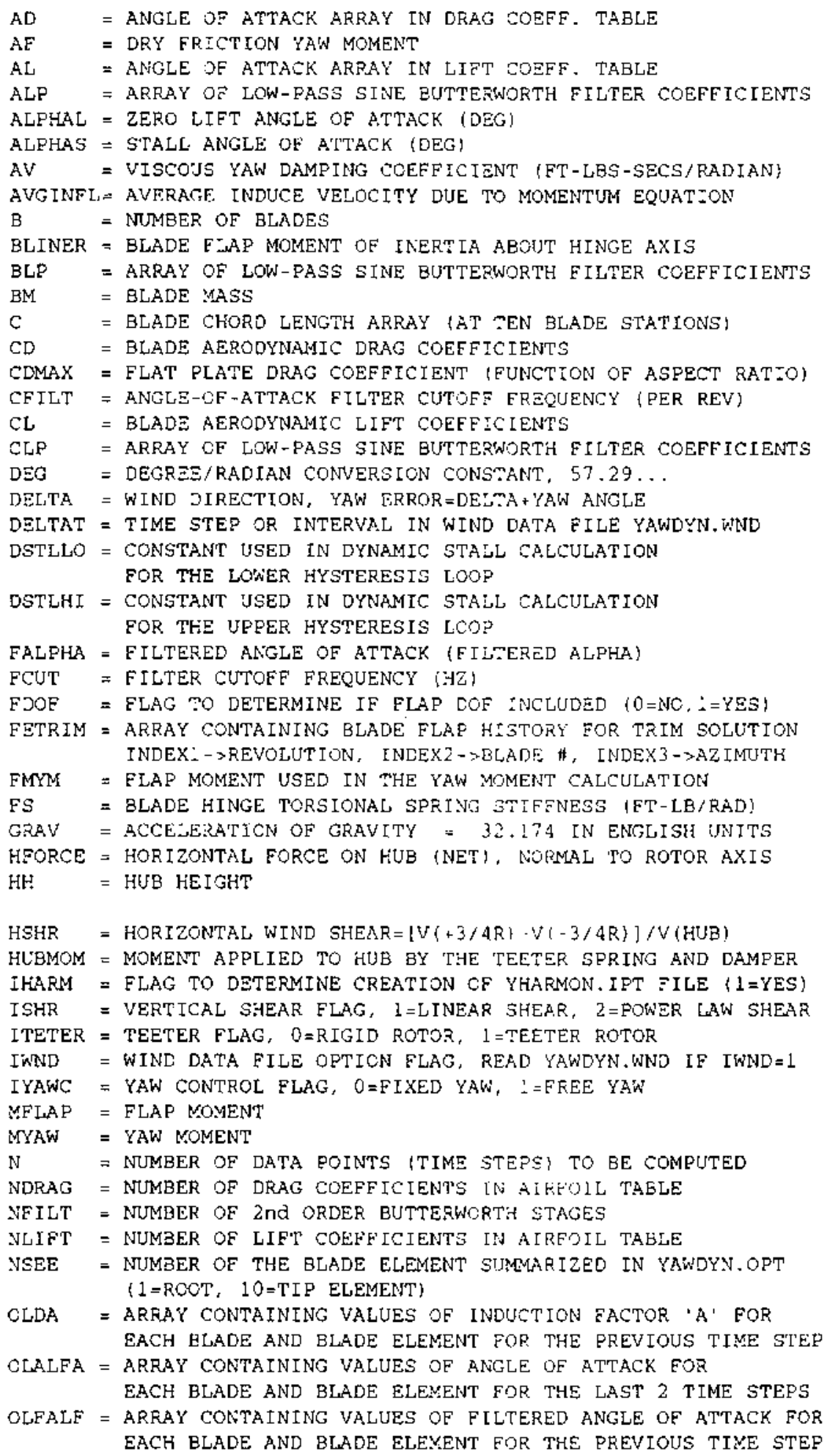




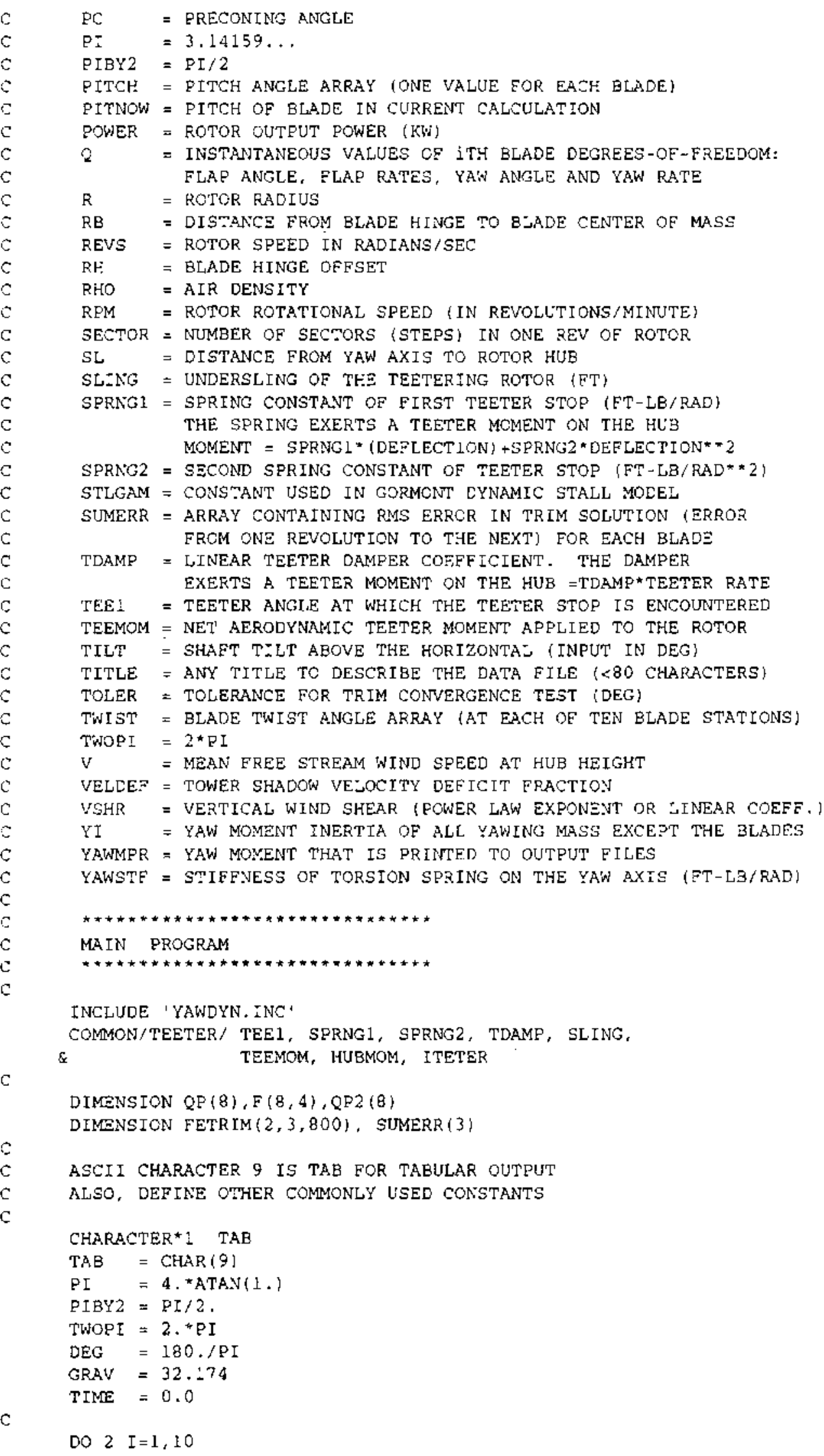




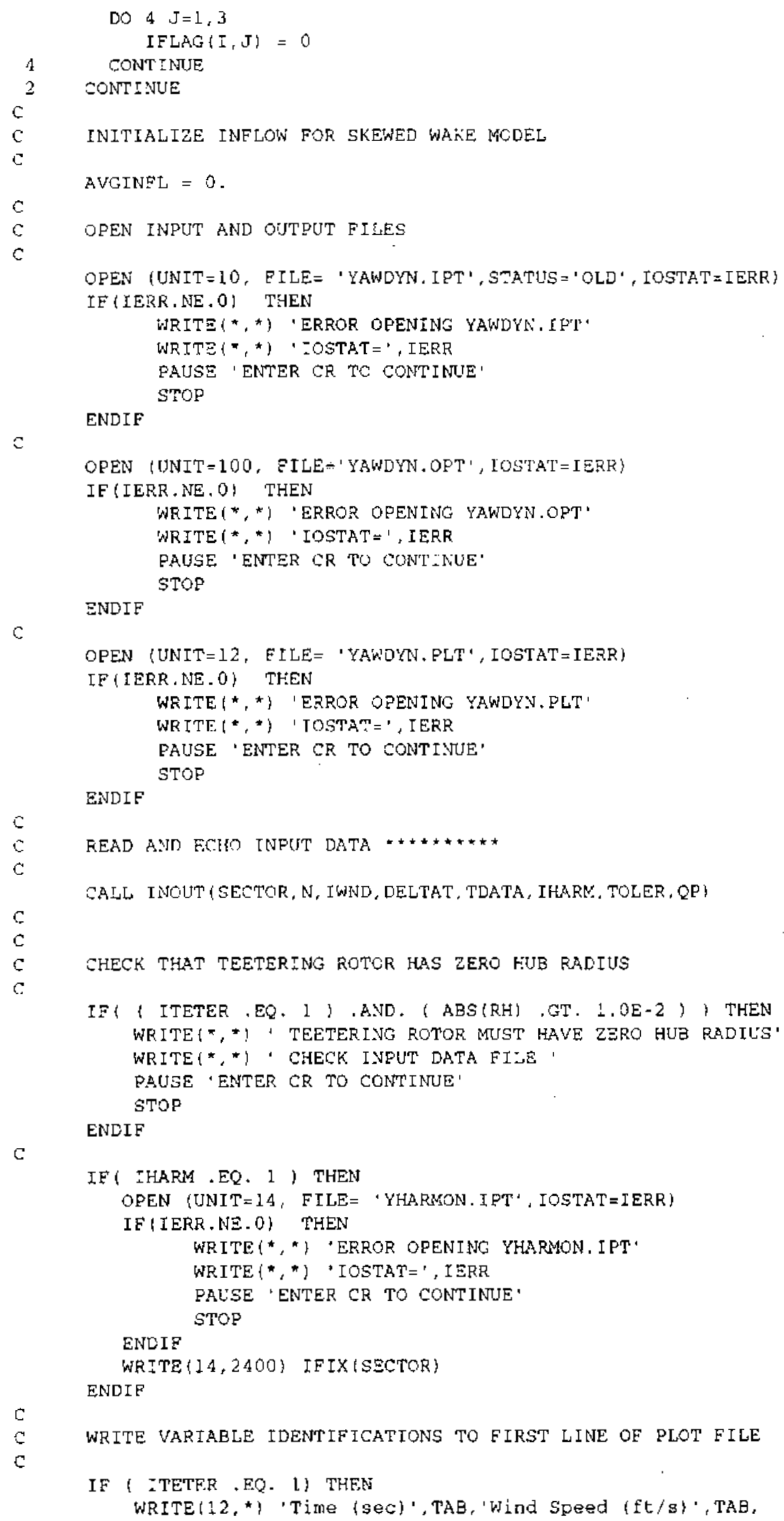




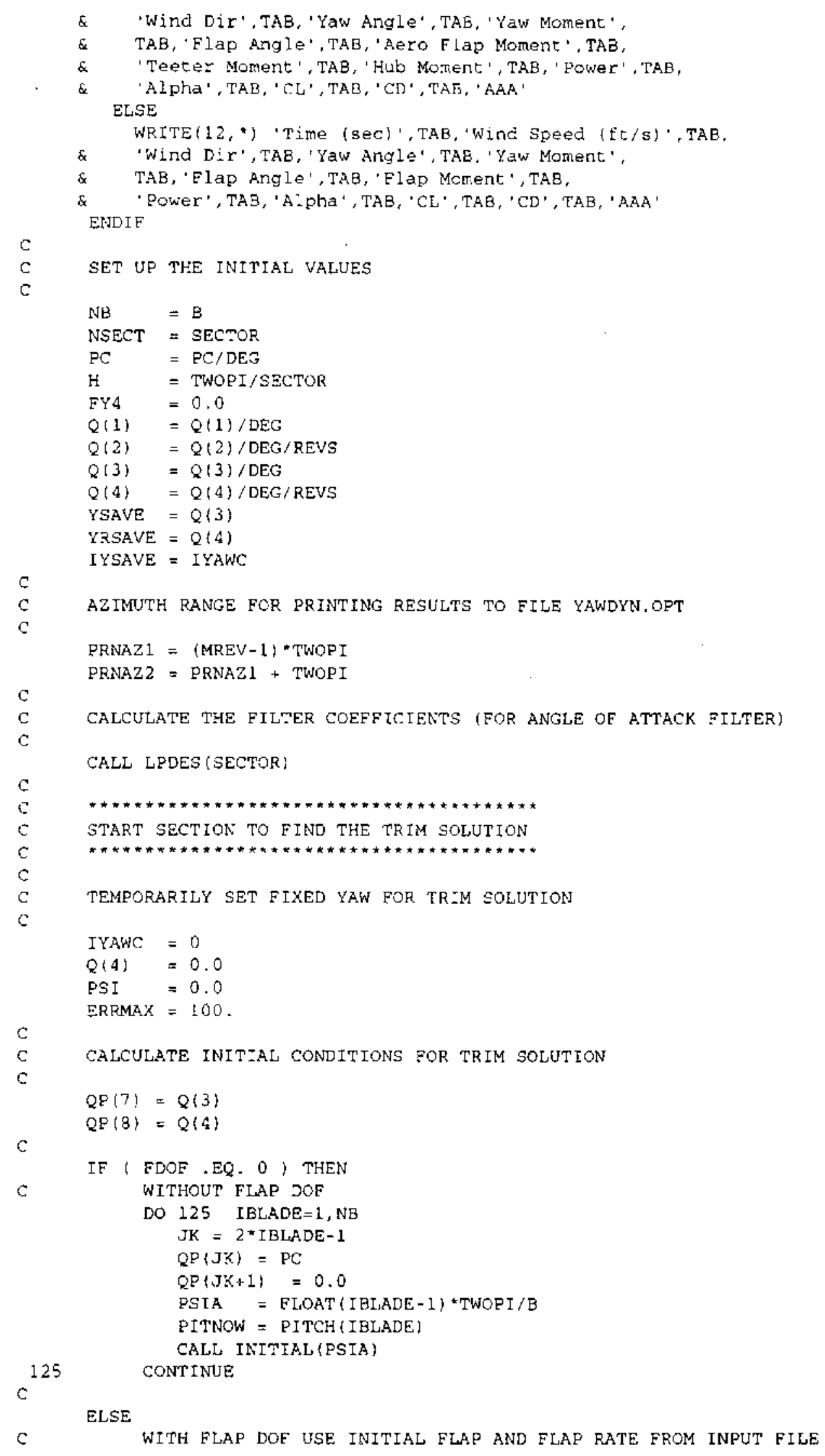




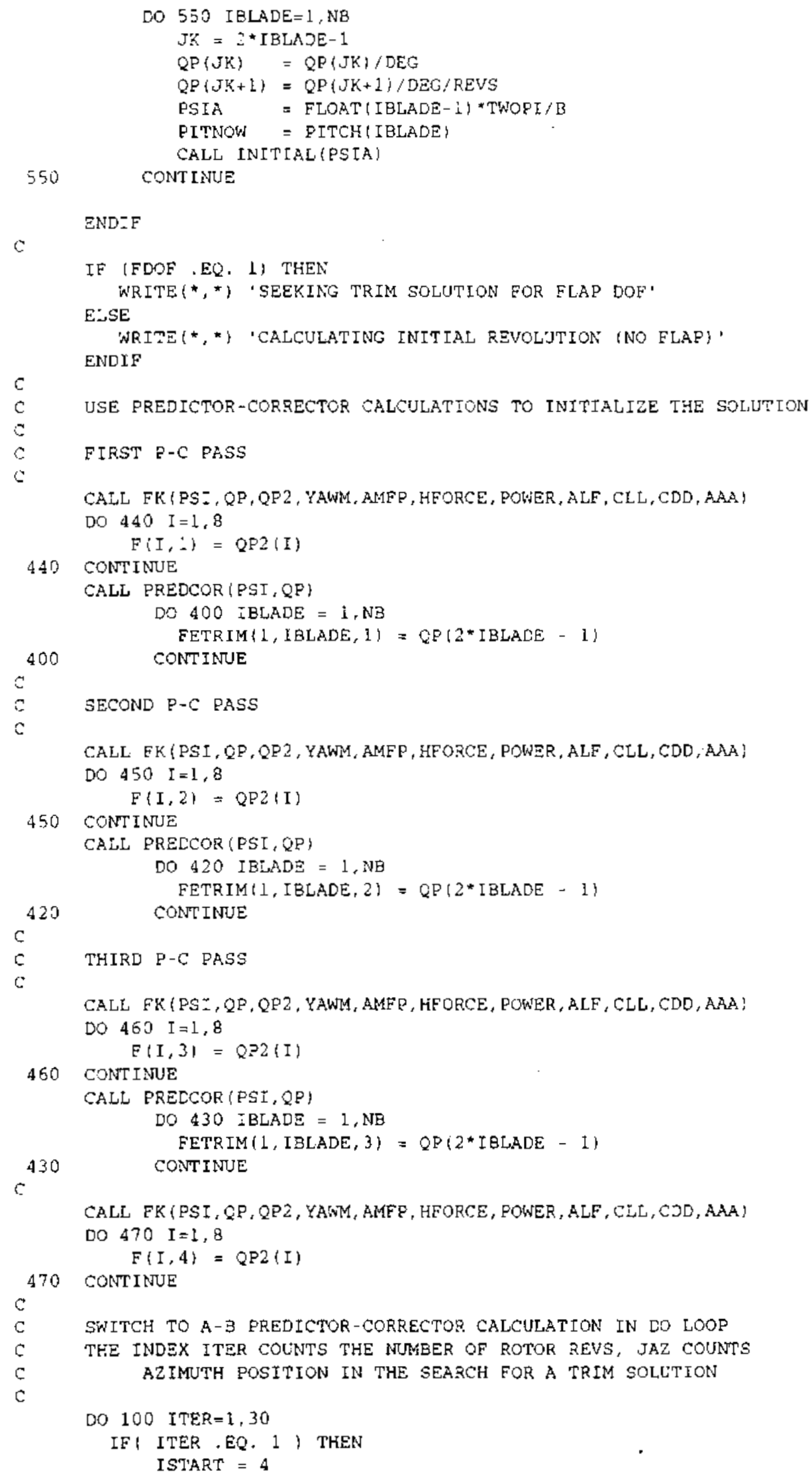




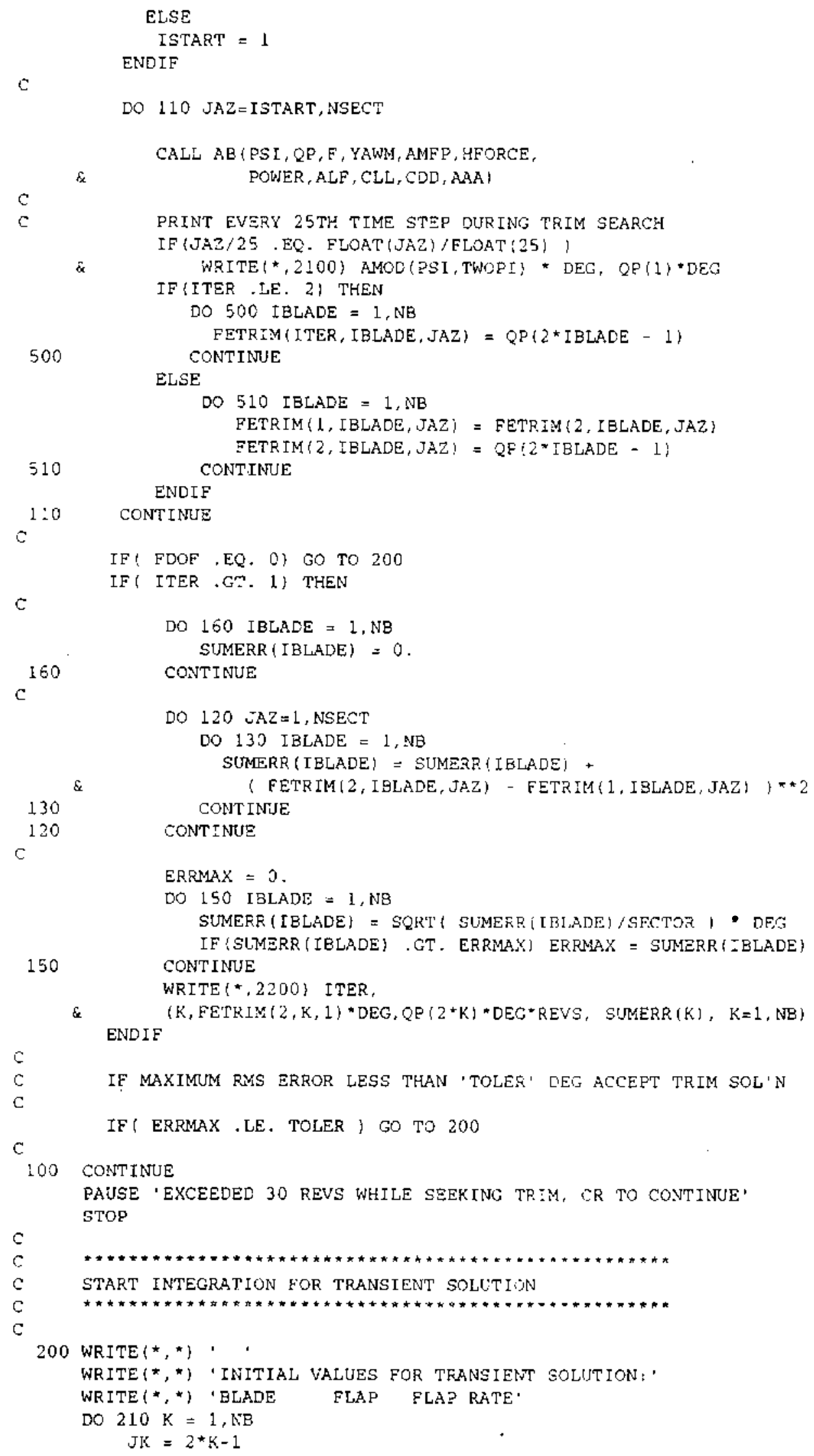

C 


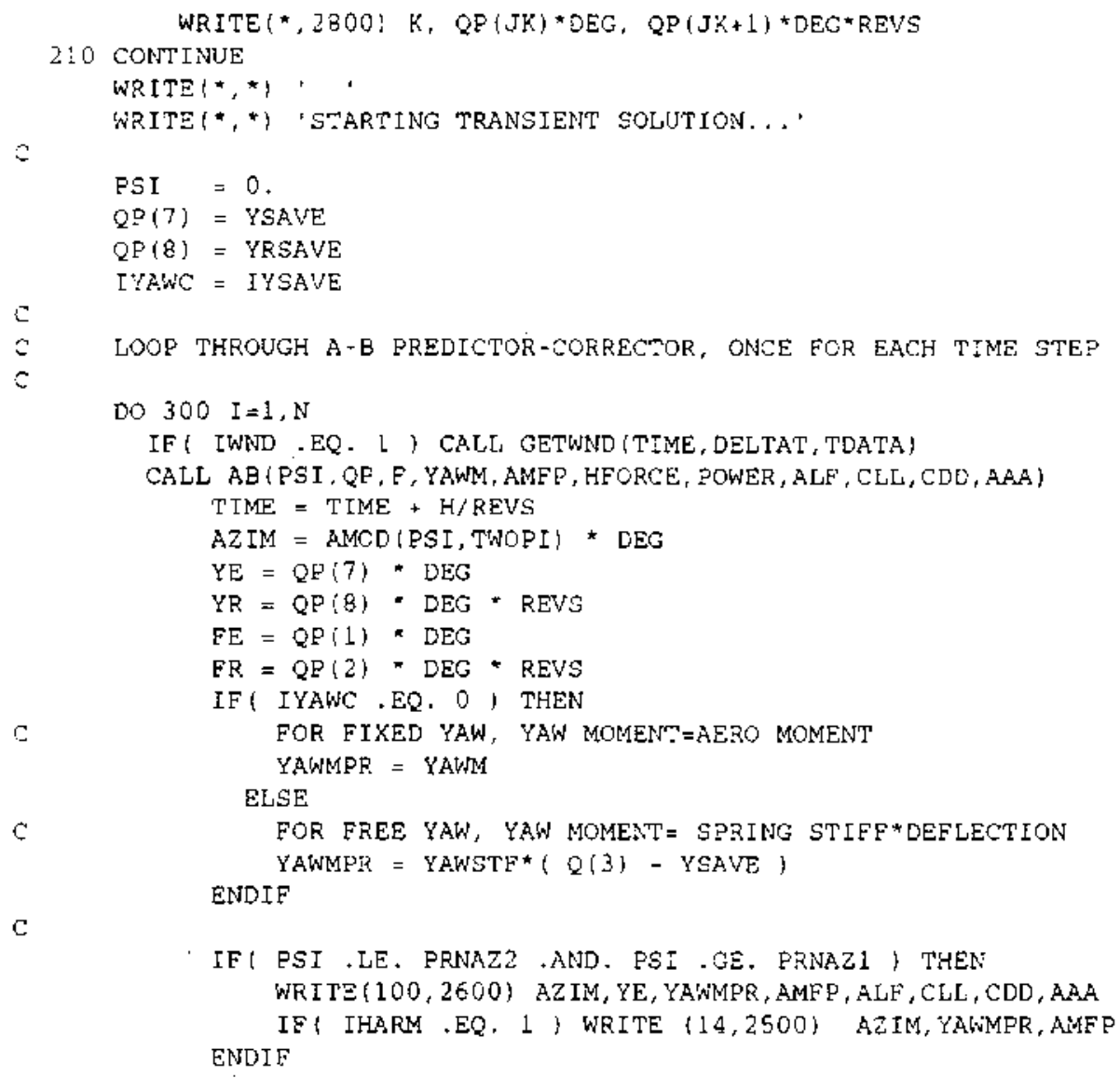

$\mathrm{C}$

IF! PSI , LE. PRNAZ2 , AND. PSI . GE. PRNAZ1 , THEN WRI TE $(100,2600)$ AZIM, YE, YANMPR, AMFP, ALF, CLL, CDD, AAA ENDIF IE IHARM .EQ. 1$\}$ WRITE $(14,2500)$ AZIM, YAWMPR, AUFP

G

IF (I/IPRINT .EQ. FLOAT(I)/FLOAT (IPRINT) I THEN WRITE $(*, 2000)$ TIME, AZIM, YE, YR, EE, ER 
IF( IWNL .EQ. 1 , CLOSE (11)

CLOSE $\{12\}$

IF I IHARM .EQ. 1 , CLOSE (14)

CLOSE $\{100\}$

PAISE 'EINISHED, ENTER CR TO CONTINUE'

$c$

STOP

END

$\mathrm{C}$ 
c

C

C $F 21=F M / A 2-\left\{E 1^{*} Q\{4\}+E 2 j^{*} Q(4)-E 3-E 4-E 5^{*} \operatorname{SPSI} F Y 4\right.$

RETURN

END

$\mathrm{C}$

AERO CALCULATES YAW MOMENT, H FORCE, POWER, FLAP MCMENT

SUBROUTINE AERO (YAW, YAERO, HFORCE, POWER, AMFP, PST, $\varepsilon$ PK, PK2, ALF, CLL, LDD. AAA)

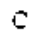

INCLUDE - YAWDYN. INC

COMMON/TEETER/TEEL, SPRNG1, SPRNG2, TDAMP, SIING, \& TEEMOM, HUBMOM, ITFTER

C

DIMENSION PK (B), PK2(8)

C

$\begin{array}{ll}Q(3) & =\text { PK }(7) \\ Q(4) & =\text { FK }(8) \\ \text { YAWM } & =0 . \\ \text { YAERO } & =0 . \\ \text { HLOHCE } & =0 . \\ \text { POWER } & =0 . \\ \text { FY } & =0 . \\ \text { F2 } & =0 .\end{array}$

C

AVGINFL1 $=\mathrm{C}$.

DO 10 IBLADE $=N B, 1, \cdot \cdot 1$

$J=I B L A D E * 2-1$

$K=J+1$

$Q(1)=E K(J)$

$Q(2)=P K(K)$

PSIA = PSI + FUOAT $(I B L A D E-1) *$ TWOPI/B

SPSIA = SIN (PSIA)

CPSIA $=\operatorname{COS}($ PSIA)

PITNOW = PITCH:IBLADE!

C

CALL BLDFM(FN, FT, FM, ZM, AVEL, PSIA, ALF, CLL, CDD, AAA)

C

AVGINEL1 = AVGINFL1 + AVF.I.

HFORCE = HFORCE + F* CPSIA

POWER $\quad=$ POWER + (F\%*RH + ZM $)$

$\mathrm{C}$

THE FLAP MOMEN' TRANSMITTED BY THE SPRENG CONTRIBUTES $\because$ THE RIGID ROTOR YAW MOMENT

IE ( (FDOF .EQ. 1) , AND. (ITETER . EQ. O) ) THEN FMYM $=$ ES $*(Q(1)-P C)$

ELSEIF ( (FDOE, EQ. C) A.ND. IITETER.EQ. O। THEN 


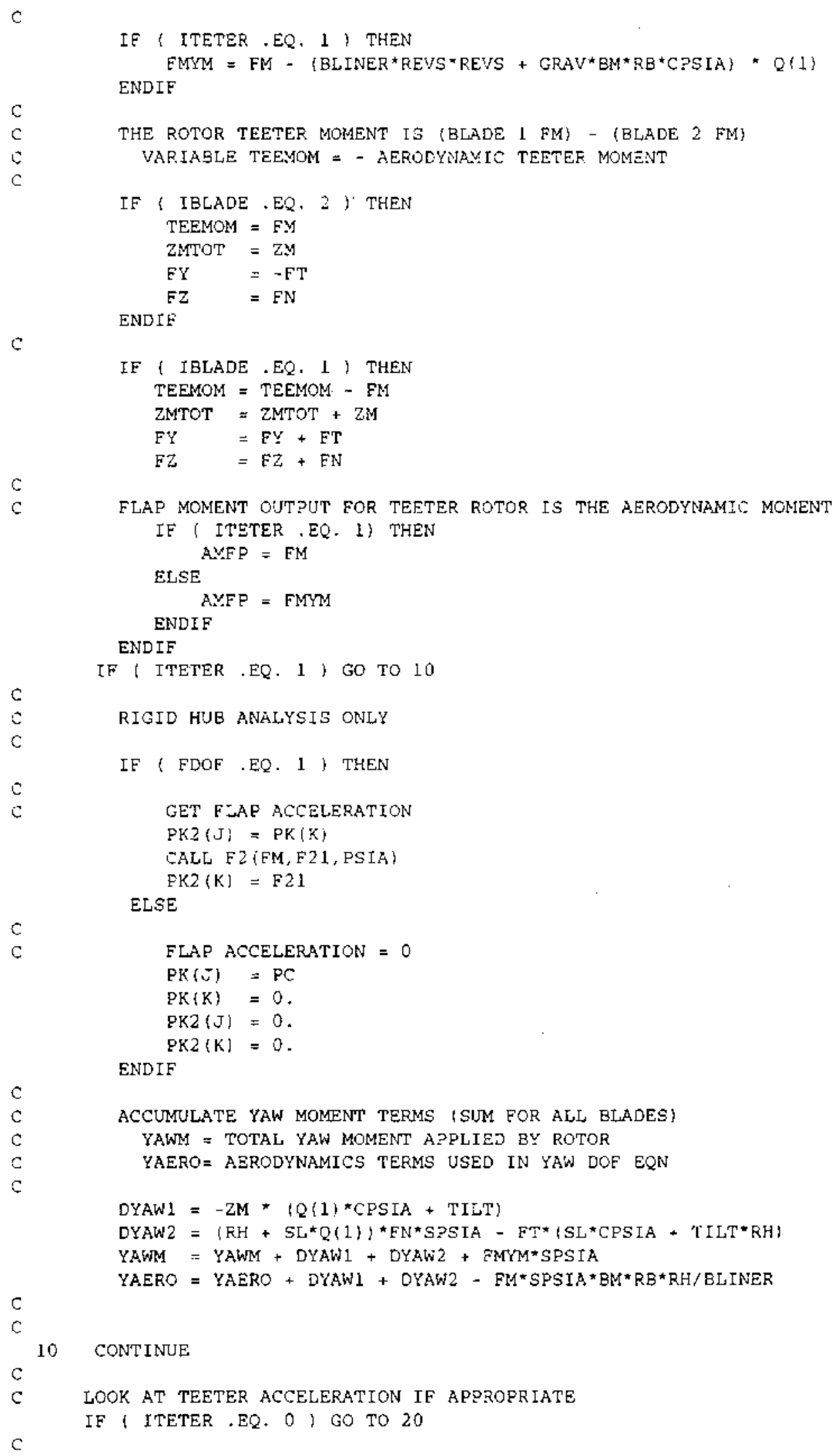

FLAP MOMENT OUTPUT EOR TEETER ROTOR IS THE AERODYHAMIC MOMENT IE ( ITETER , EQ . 1) THEN

C 
GET THE TEETER ACCELERATION FROM THE NE'T MOMENT AND THE APPROPRIATE AERODYNAMIC YAW MOMENT

$$
\begin{aligned}
& Q(1)=\mathrm{PK}(1) \\
& Q(2)=\mathrm{PK}(2)
\end{aligned}
$$$$
\operatorname{PK} 2(1)=\operatorname{PK}\{2\}
$$

TEETER $=Q(1)-\mathrm{PC}$

CPSI $=\operatorname{COS}\{$ PSI $\}$

YAERO $=-E{ }^{*}\{S L-S L I N G) * C P S I+E Z * S L * T E E T E{ }^{*}{ }^{*} I N\{P S I\}$

CALL F2 \{FM, F21, PSI

$P K 2(2)=E 21$

YAW MOMENT RESULTS FROM THE HCRIZCNTAL FORCE, TORQUE, AND THE TEETER STOP MOMENT (HUBMOM) (USED FOR FIXED YAW ONLY)

YAWM $=$ YAERO + FUBMOM * SIN (PSI)

POWER OUTPUT IN KILOWATTS

20 FOWER = FOWER * REVS *.001356

AVGINFL $=$ AVGINF II $/ 3$.

RETURN

END

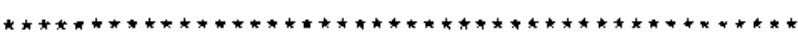

BLDFM CATCULATES THE FORCES AND MOMLNTS FOR

THE BLADF. AT AZTMUTH ANGLE PSL .

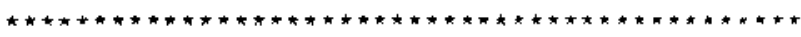

SUBROUTINE BLDFM \{FN, FT, FM, ZM, AVEL, PSI, ALE, CSL, CDD, AAA

INCLUDE 'YAWDYN. INC'

$E N=0$.

FT $=0$.

$Y M=0$.

$Z M=0$.

AVEL $=0$.

AVEL IS THE INDJCED VELOCITY IN NORMAL

DIRECTION EY US:NG MOMENTUM EQUATION.

VELD CALCULATE THE VELOCITIES RELATIVE TO THE ROTOR DISK

CALL VELO (SDEL, CDEL, ANGELW, NTEST)

$c$

DO $10 \mathrm{~J}=1,10$

$\mathrm{X}=(\mathrm{J} * .1-.05){ }^{*} \mathrm{R}-\mathrm{RH}$

IF( X .LE, 0.$)$ GO TO 10

CALI VEL (VY, VZ, $X, E S I)$

CALL VIND (A, J, X, PSI, VY, VZ, VN, VT)

VNORM $=V N /(1,-A)$

AVEL $=$ AVEL + VNORM*A*COS $(Q(1\}) * T W O P{ }^{*} \times *(0.1 * R)$

C

IF ( NTEST ,EQ, 1 ) THEN

CALL VNMOD (VN, $X, A, A X Y, P S I$, SDEL, CDEL, ANGELW\}

ELSE

$A X Y=A$

ENDIE 


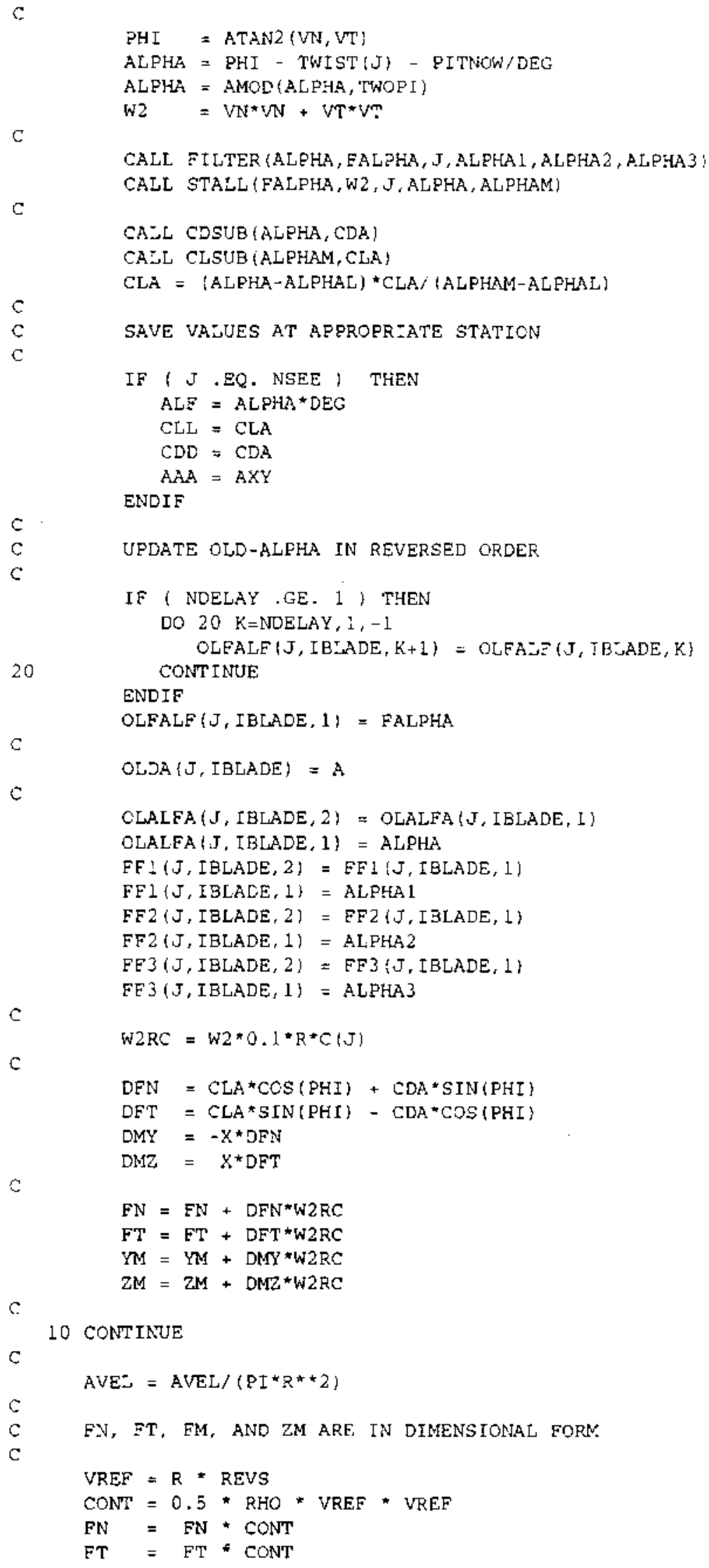




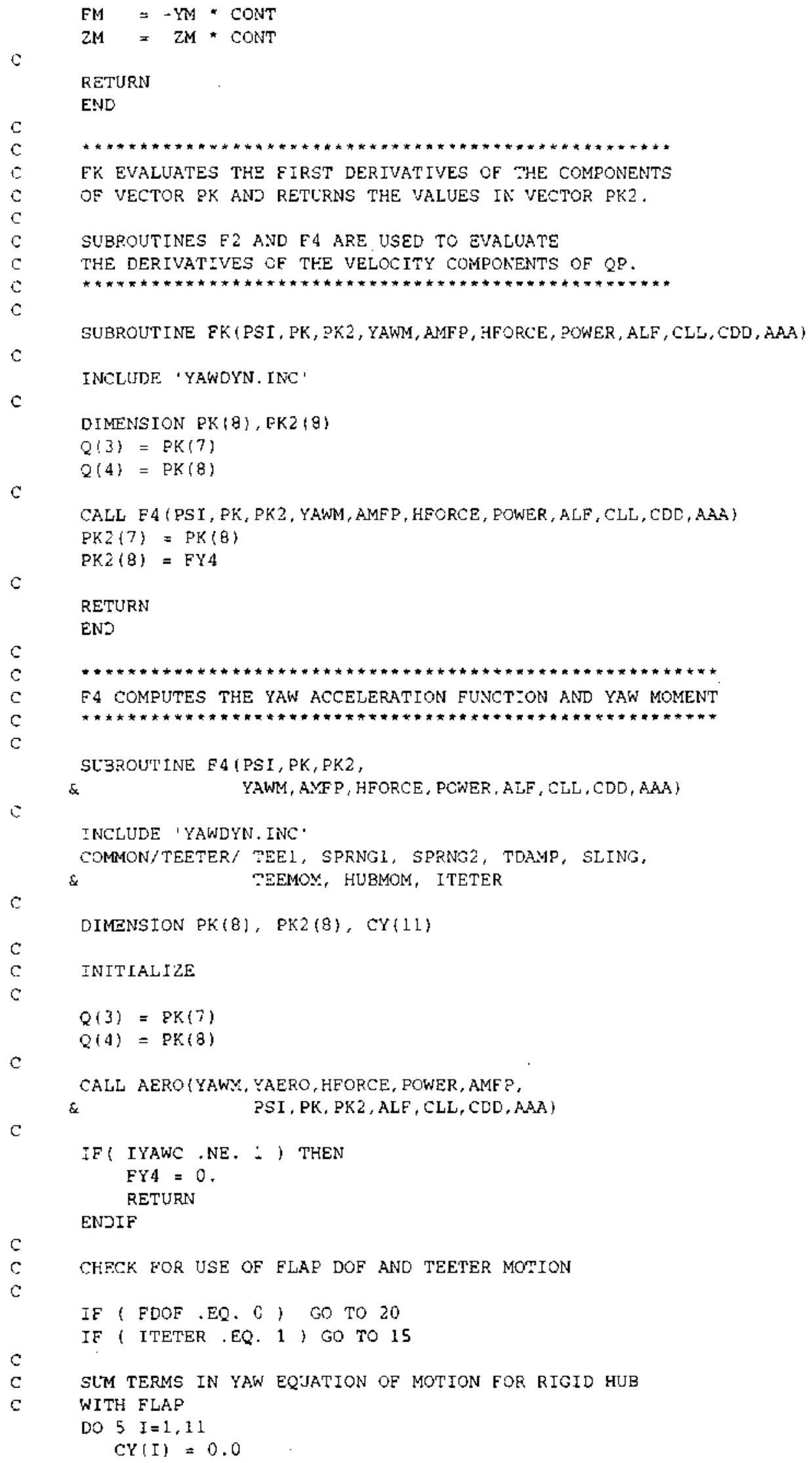

$c$

CALL AERO \{YAWN, YAERO, HFORCE, POWER, AMF?,

$\mathrm{C}$ \& PSI , PK, PK2, ALE, CLL, CDD, AAA)

IE ( IYAWC .NE. : ) THEN

FY4 $=0$. RETURN

ENDIF

c

CHFCK FOR USE OF FLAP DOF AND TEETER MOTION

IF ( FDOF.EQ. C) GO TO 20

IF ( ITETER.EQ. 1 ) GO TO 15

$\mathrm{C}$

SLM TERMS IN YAW EQJATION OF MOTION FOR RIGID GUB WITH FLAP

DO $5 I=1,11$

$C Y(I)=0.0$ 


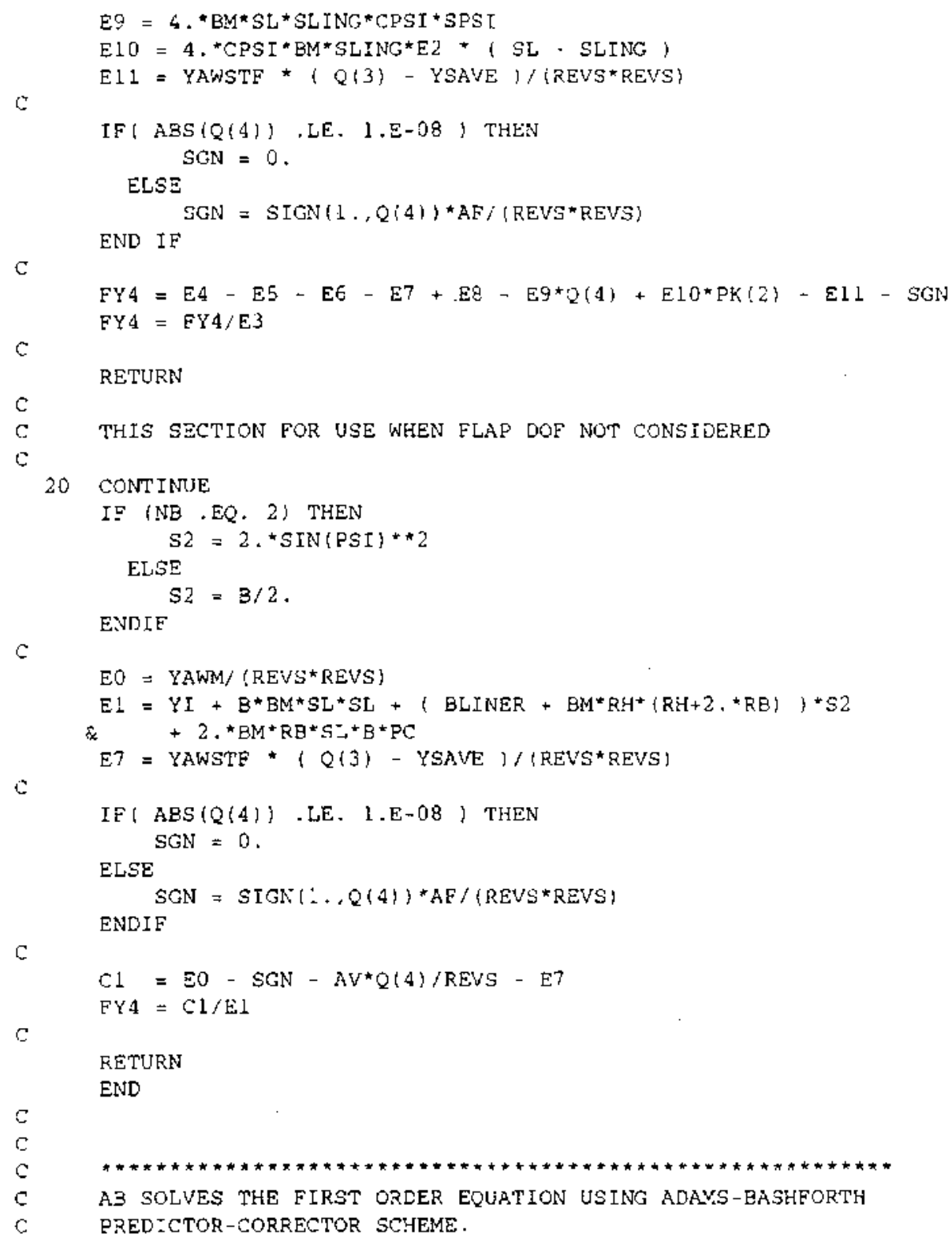

THE MATRIX F CONTAINS THE FREVIOUS FOUR SETS OF DERIVATIVE FUNCTIONS EVALUATED BY SUBROUTINE FK. 'THE DEGREES-OF-FREEDCM CONTAINED IN VECTOR QP ARE ADVRNCED

ONE STEP SIZE, H, AND THE MATRIX F IS SHIFTED TO STORE THE NEN SET OF DERIVATIVE FUNCTION VALUES DURING EACH RUN OF AB.

VARIABLES :

CK = CORRECTOR TERM (LOCAL TO THIS SUBROUTINE)

PK = PREDICTOR TERM (PASSED TO FK FOR EVALUATION OF FLNCTION $E$ )

QP = SOLJTEON VECTOR (BETA1, BETA1', BETA2, BETA2', BETA3, BETA 3' , GAMMA, GAMMA')

$\vec{F}=$ RHS OF 8 GOVERNING EQUATLONS, EVALUATED AT LAST FOUR TIME STEPS (THE LAST FCUR VALUES OF PK2।

PK2 = PK PRIME MOST RECENT VALUE OF F, THAT IS, THE DERIVATIVE OF PK BETA1 ', BETA1 ' ' BETA2', BETA2 ' ', BETA3', BETA3 ' ', GAMMA', GAMMA ' ' 
SUBROUTINE AB (PG , QP, F, YAWM, AMFP, HFORCE, POWER, ALF, CLL, CLD, AAA )

C INCLUDE 'YAWDYN. INC'

COMMON/TEETER/ TEEL, SPRNGL, SPNNG2, TDAMP, SLING,

\&

TEEMOM, HUBMOM, ITET $\Xi R$

$c$

DINENSION $Q P(8), F(3,4), P K(8), C K(8), \operatorname{PK} 2(8)$

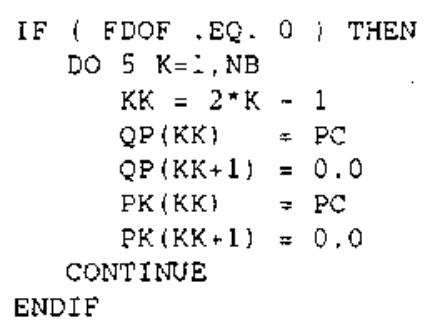
ENDIF

C

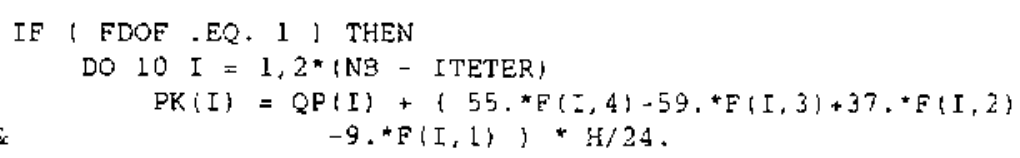


$F(I, 4)=\operatorname{PK} 2(I)$

CONTINUE

RETURN

END

$C$

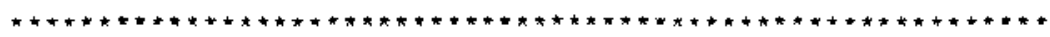

PREDCOR SOLVES THE EQUATION USING PREDICTOR-CORRECAOR METHOD

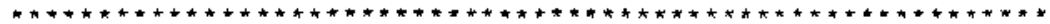

THE NEW WAUUES ARE RETURNED IN QP. SUBROUTINE

FK IS USED TO EVALUATE THE DERIVATIVE FUNCTIONS.

SUBROUTINE FREDCOR (PSI, QP)

INCLUDE 'YAWDYN. INC'

COMMON/TEETER/ TEE1，SPRNG1，SPRNG2，TLAMP, SLING,

$\mathrm{C}$ TEEMOM, HUBMOM, ITETER

c

DIMENSION QP $(8), \mathrm{QK}(8), \mathrm{QK}(8), \mathrm{TK}(8)$

IF ( FDOF .EQ. 0 ) THEN

DO $30 \mathrm{~K}=1, \mathrm{NB}$

$K K=2^{*} K-1$

$\mathrm{QP}(K K)=\mathrm{PC}$

30

$\mathrm{QP}(\mathrm{KK}+1)=0.0$

ENDIF

$\mathrm{C}$

C

PREDICTOR CALCULATION

CALL FK IPSI, QP, TK, YAWM, AMFP, HFORCE, POWER, ALF, CLL, CDD, AAA)

DO $10 I=1,2 *(\mathrm{NB}$ - ITETER $)$

$P K(I)=Q P(I)+T K(I) * H$

10 CONTINUE

IF ( ITETER .EQ. 1 ) THEN

$P K(3)=2 \cdot{ }^{*} P C-P K(1)$

$\mathrm{PK}(4)=-\mathrm{PK}(2)$

ENDIE

DO 15 I $=7,8$

$\mathrm{PK}(I)=\mathrm{QP}(I)+\mathrm{TK}(I) \cdot \mathrm{H}$

15 CONTINUE

c

C

CORRECTOR TERM

$P S I=P S I+H$

CALL FK (PSI, PK, QK, YAWM, AMFP, HFORCE, POWER, ALF, CLL, CDD, AAA)

DO $20 I=1,2 *(\mathrm{NB}$ - ITETER $)$

2C CONPINUE

$Q P(I)=Q P(I)+(Q K(I)+T K(I)) * H / 2$.

DC $25 I=7,8$

25 CONTINUE

$\mathrm{C}$

c

FORCE TEETER MOTION FOR ELADE 2 IF TEETERING ROTOR

IF (ITETER . EQ. 1 , THEN

$Q P(3)=2 . * P C-Q P(1)$

ENDIF

$Q P(4)=-Q P(2)$

RETURN

END

C SUBROUTINES USED IN YAWDYN

C ACH $6 / 5 / 91$ 


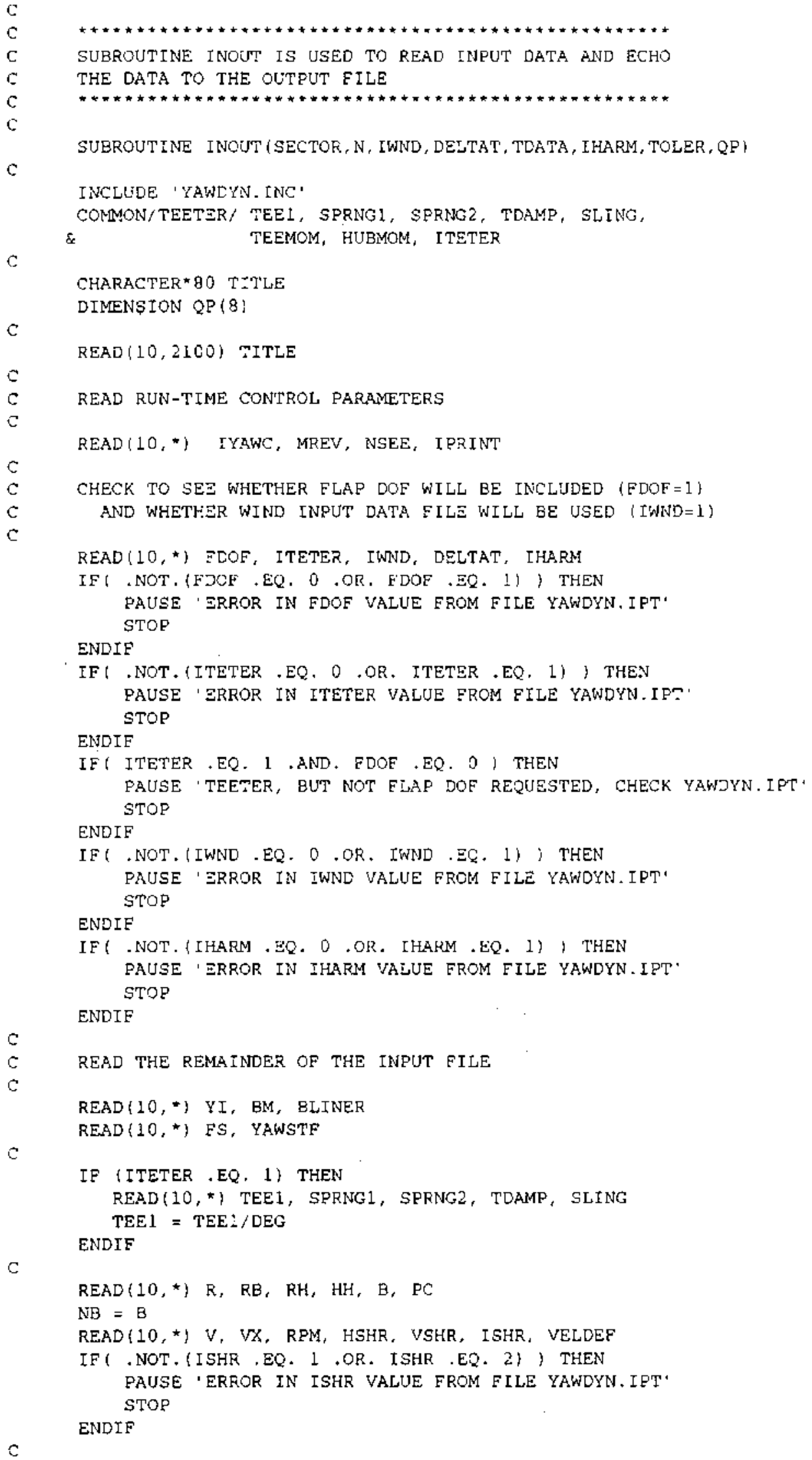




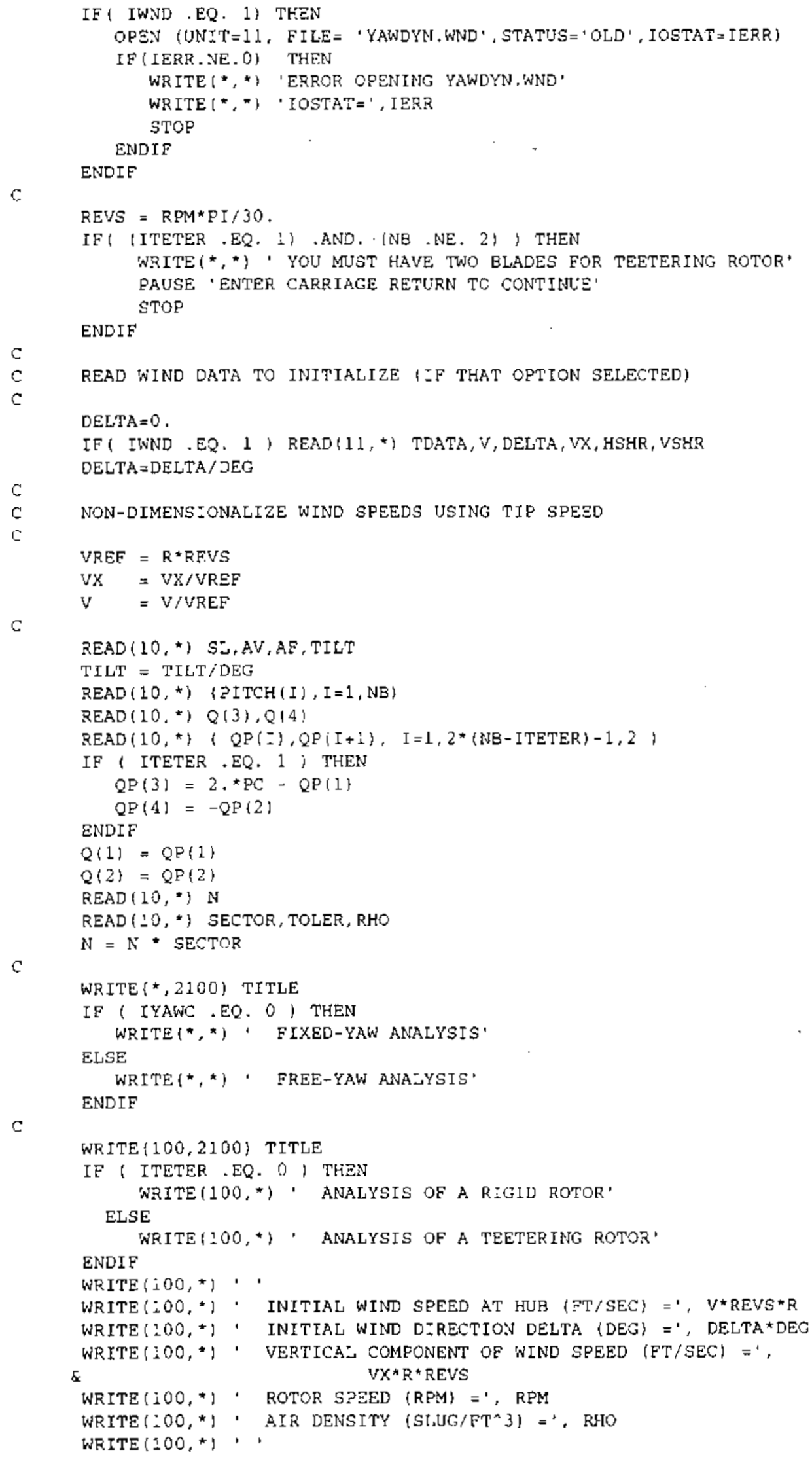




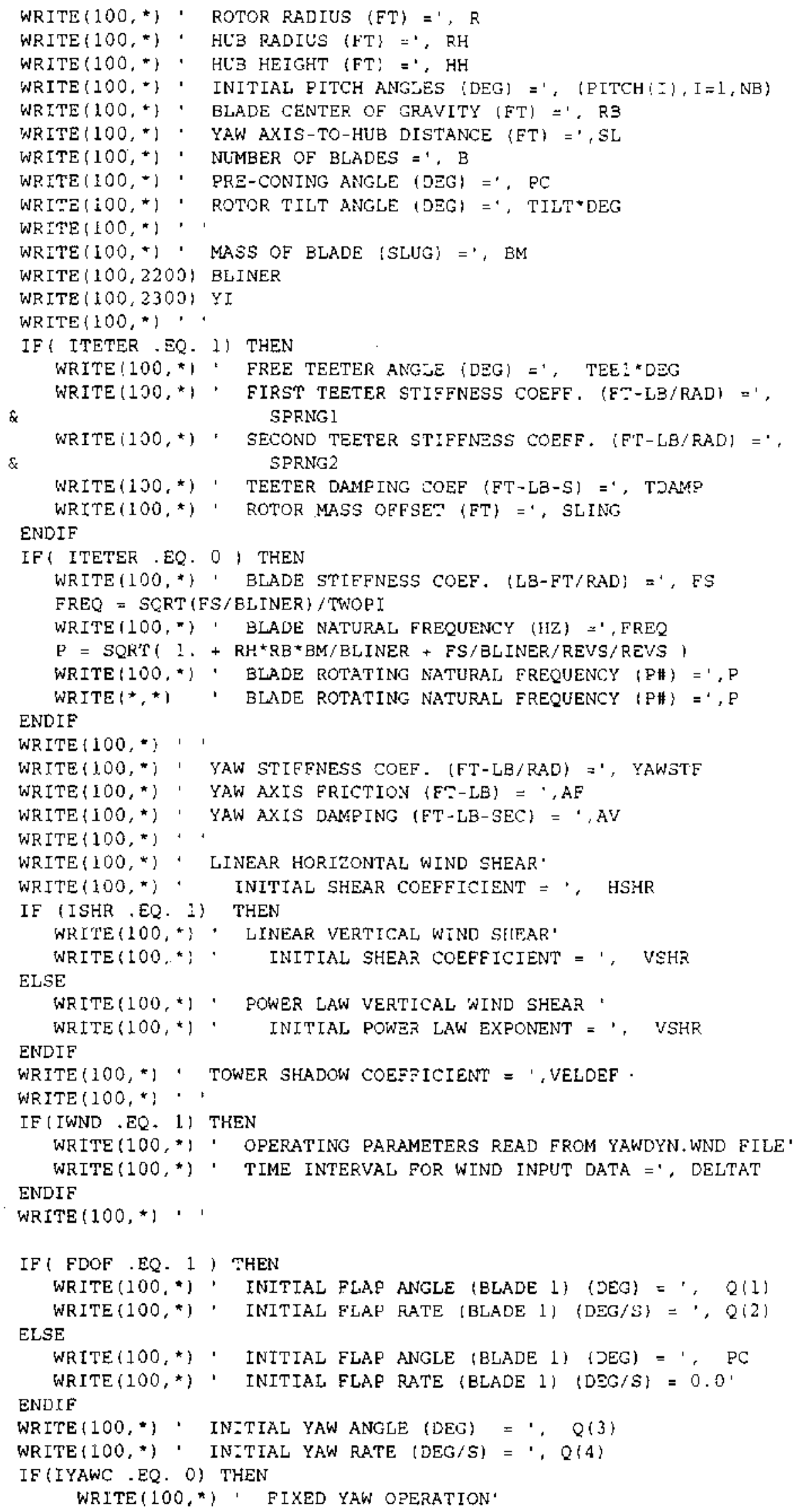




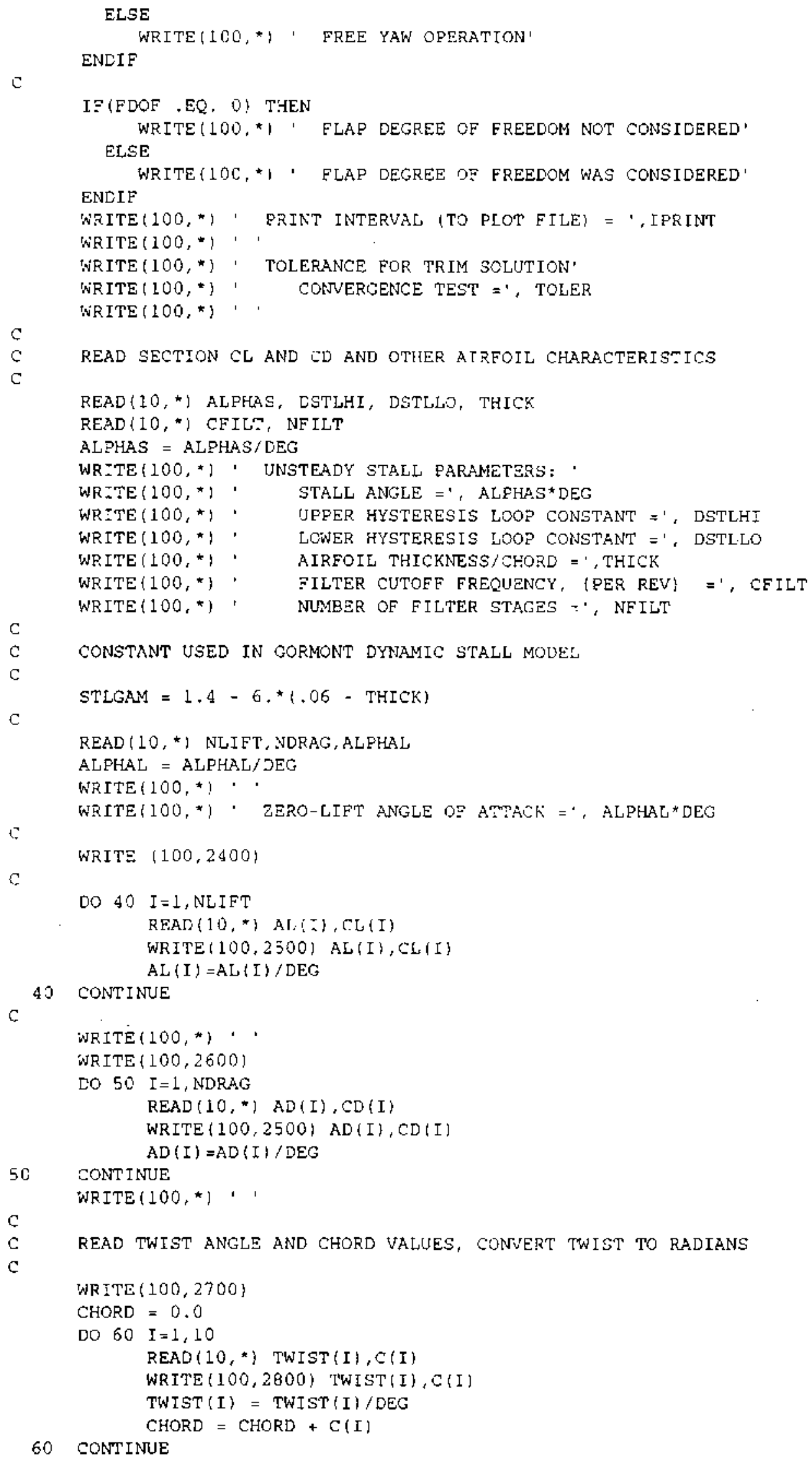


$c$

$c$

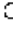

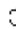

CLOSE $(10)$

WRITE $(100, *)$,

IF ( (FDOF .EQ. 1) .AND. (ITETER , EQ. O ) THEN WRITE $(100, ")$, FLAP MOMENT IS THE BLADE DEELECTYON',

$\varepsilon$ - TIMES THE SFRENG STIEFNESS'

ELSEIF ( (FDOF .EQ. 1) AND. (IMETER .EQ. 1) ) THEN WRITE $(100, *)$ ' FLAP MOMENT IS THE APPLIED AERODYNAMIC',

$\varepsilon$

ELSE

WRITE $(100, *)$ ' FLAP MOMENT IS THE APPLIED AERODYNAMIC, ,

ENDIF

¿

IF I IYAWC,EQ. I I THEN

WRITE $(100, *)$ ' YAW MOMENT IS YAW DEELECTION*VAW STIFFNES3

ELSE

WRITE $(100, *)$ " YAW MOMENT IS APPLIED AERODYNAMIC MOMENT" ENDIE

WRITE $\{100,2900\}$ MREV, NSEE

WRITE $(100, *)$,

WRTTE $\{*, *) \cdot$

WRITE $(*, *)$ 'RUNNING $1, N$, ' POINTS

WRITE (*, *) 'NITH ', SECTOR,' POINTS PER REVOLUTION'

TOFF $=$ TWOPI *N/SECTOR/REVS

WRITE $(*, *)$ 'TOTAL TIME DURATION SIMUSATED (SEC) $=1$, TOEF

WRITE $(*, *) \cdot$

2100 FORMAT (A)

2200 FORMAT (' BLADE FLAP MOMENT OF INERT:A (SLUG*ET^2) =',F10.1)

2300 FORMAT (" NACELLE MOMENT OF INERTIA (SLUG*FT^2) $=1, F: 0.1$ \}

2400 gCRMAT $(/, \cdot$ AIRFOIL CHARACTERISTICS:,$/, '$ ANGLE OF ATTACK(DEG)

\& LIFT COEF , )

2500 EORMAT $(4 \mathrm{X}, \quad 510.4,8 \mathrm{X}$, FIO.4)

2600 FORMAT $(/, '$ AIRFOIL CHARACTERISTICS:,$/, \cdot$ ANGLE OF ATTACK(DEG)

\& DRAG COEF.

2700 FORMAT $/ /$, TWIST ANGLE CHORD $* / *$, (DEG) (ET) $1 / 1$

2800 FORMAT $(1 \mathrm{X}, \mathrm{F} 10,4,4 \mathrm{X}, \mathrm{F} 7.4)$

2900 FORMAT $/ / \cdot$ DATA FOR CYCLE NUMBER', I3,', BLADE E'JEMEN: 1, I2, \& $1^{\circ}$ PSI YAW YAWMONENT',3X,

\& 'FLAPMOMENT ALPHA CL CD A'/

$\&$ DEG DEG FT-LB FT-LB

C

RETURN

END

SUBROUTINE INIMIAL

CALCULATES INIMIAJ VALUES OF ANGLE OF ATTACK FOR ONE BLADE TO INITIALIZE THE FJLTER 'MEMORY'

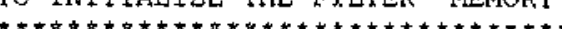

SUBROUTINE INITIAL (PSI) 


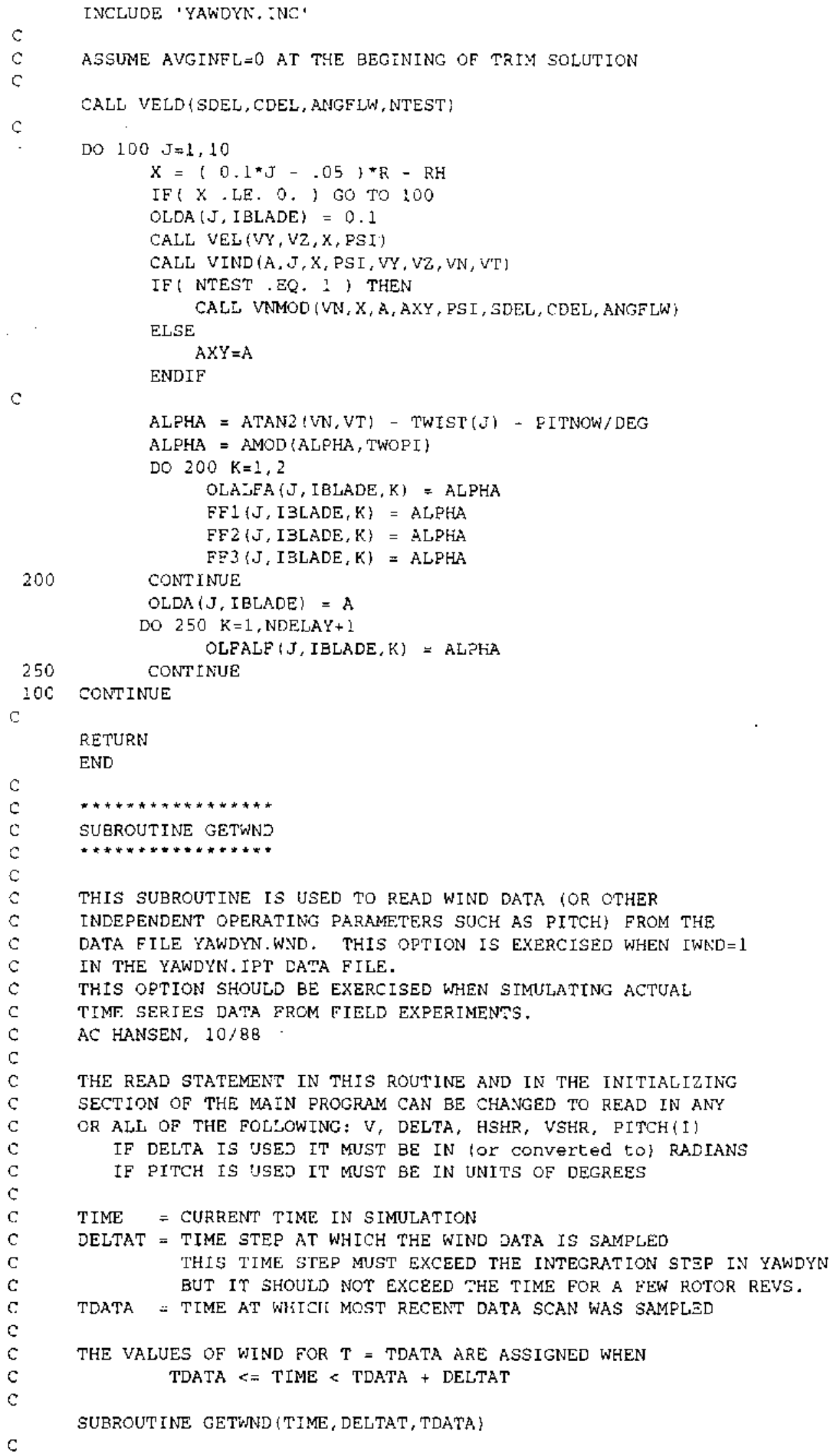




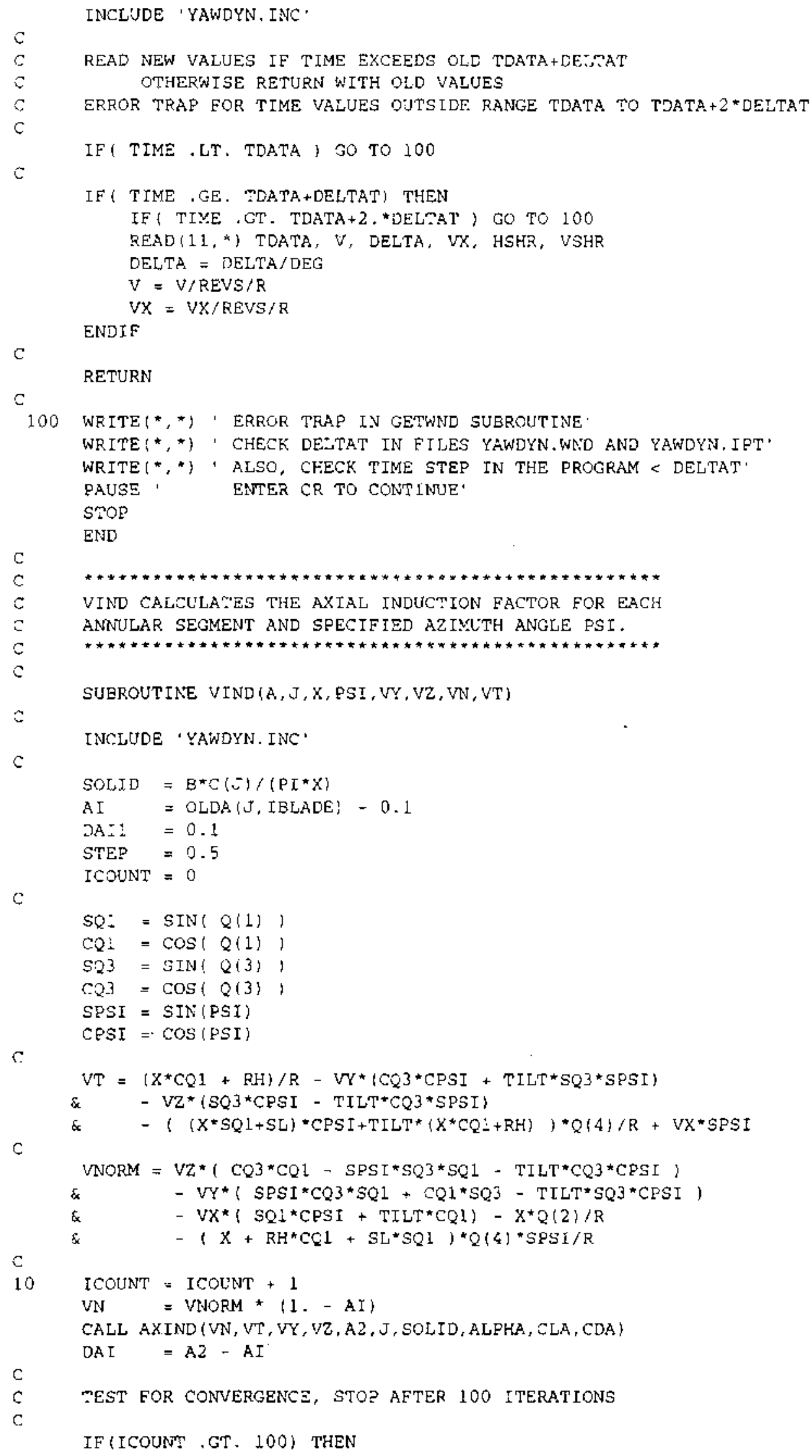




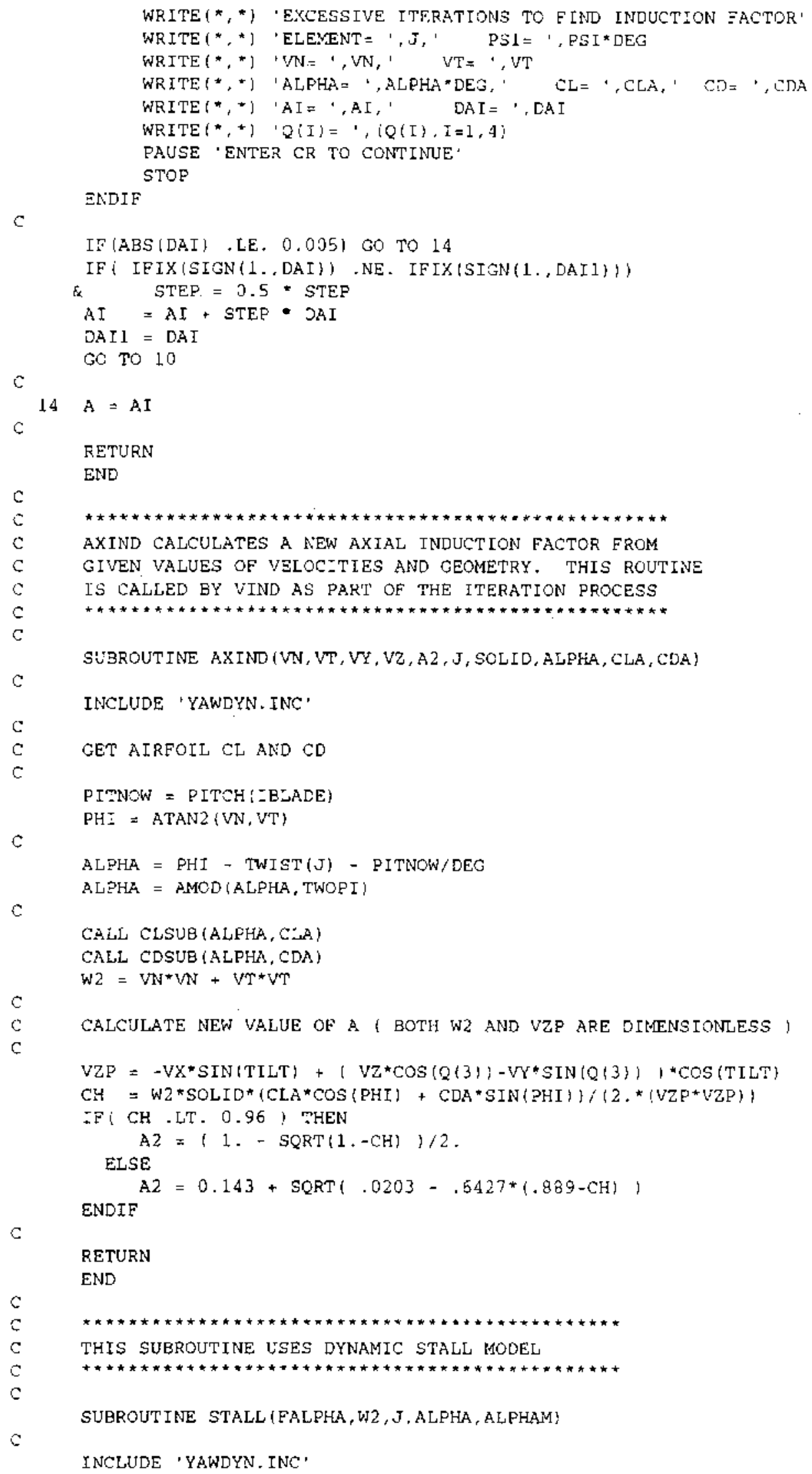


C

C

$G$

$\mathrm{c}$

$c$
JSING PREVIOJS ANGLE OF ATTACK TO CALCULATE ALDOT

TO COMPENSATE THE DELAY DUE TO EILTER

IF ( NDELAY .EQ. 0 ) THEN

ELSE

ALDOT $=\mid$ FALPHA - OLFALE $\{J, I B L A D E, 1\} \mid * R E V S / H$

NDELAYI = NDELAY + 1

ALDOT $=$ (OLFALF (J, IBLADE, NDE:AY)

ENDTF

- OLFALF (J, IBLADE, NDELAY1) ) * REVS/H

IF ( ALPHA . GE. ALPHAS) IFLAG (J, IBLADE) $=1$

IF $\{$ ALFHA . LT. ALPHAS .AND. ABS \{RLDOT) ,LE. I.E-6,

$\& \quad \operatorname{IFLAG}(\mathrm{J}$, 「BLADE $)=0$

IF( IFLAG (J, IBLADE) .EQ. 1 , THEN

USING DYNAMIC STALL MODEL

$\mathrm{UR}=\operatorname{SQFT}\{$ W2 $\} *$ REVS*R

$S T=C(J) * A L D O T /(2 * * U R)$

$S T=\operatorname{SQRT}(A B S(S T)) * \operatorname{SIGN}\{1 \ldots$ ALDOT $)$

IE ( ALDOT*ALPHA. .GT. 0.) THEN $X K=D S T L H I$

ELSE

$X K=$ DSTLLO

ENDIF

USE UNFILTERED ANGLE OF ATMACK

ELSE

ALPHAM = ALPHA $-X K * S T L G A M * S T$

NOT USING DYNAMIC STALL

ALPHAM $=$ ALPHA

ENDIF

RETURN

END

LOWPASS BUTTERWCRTH DIGITAL FILTER DESIGN SUBROUTINE SOURCE FILENAME LPDES

TTE SUBROUTUNE WTLT RETURN THE C REALIZATION OF A MULTIPLE SECTION LOWFASS FILTER. TFE KEh SECTION HAS THE FOLLOWING TRANFER EUNCTION:

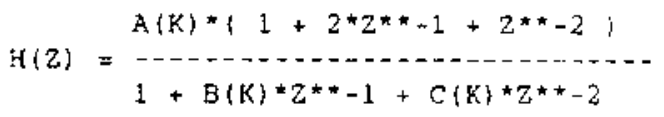

IHUS, IF $F(M)$ AND $G(M)$ ARE THE INPUT AND OUTPUT OF THE Kth SECTION, THE FOLLOWING DIFFERENCE EQUATION IS SATISFIED:

$G(M)=A(K) *(F\{M)+2 * F(M-1)+F(M-2),-B(K) * G(M-1) * C(K) * G(M-2)$

REFERENCE: DIGITAL SIGNAL ANALYSTS, SAMUEL D. STEARNS, HAYDEN BOOK COMPANY, INC. ROCHELLE EARK NEW JERSEY, APPENDIX C 


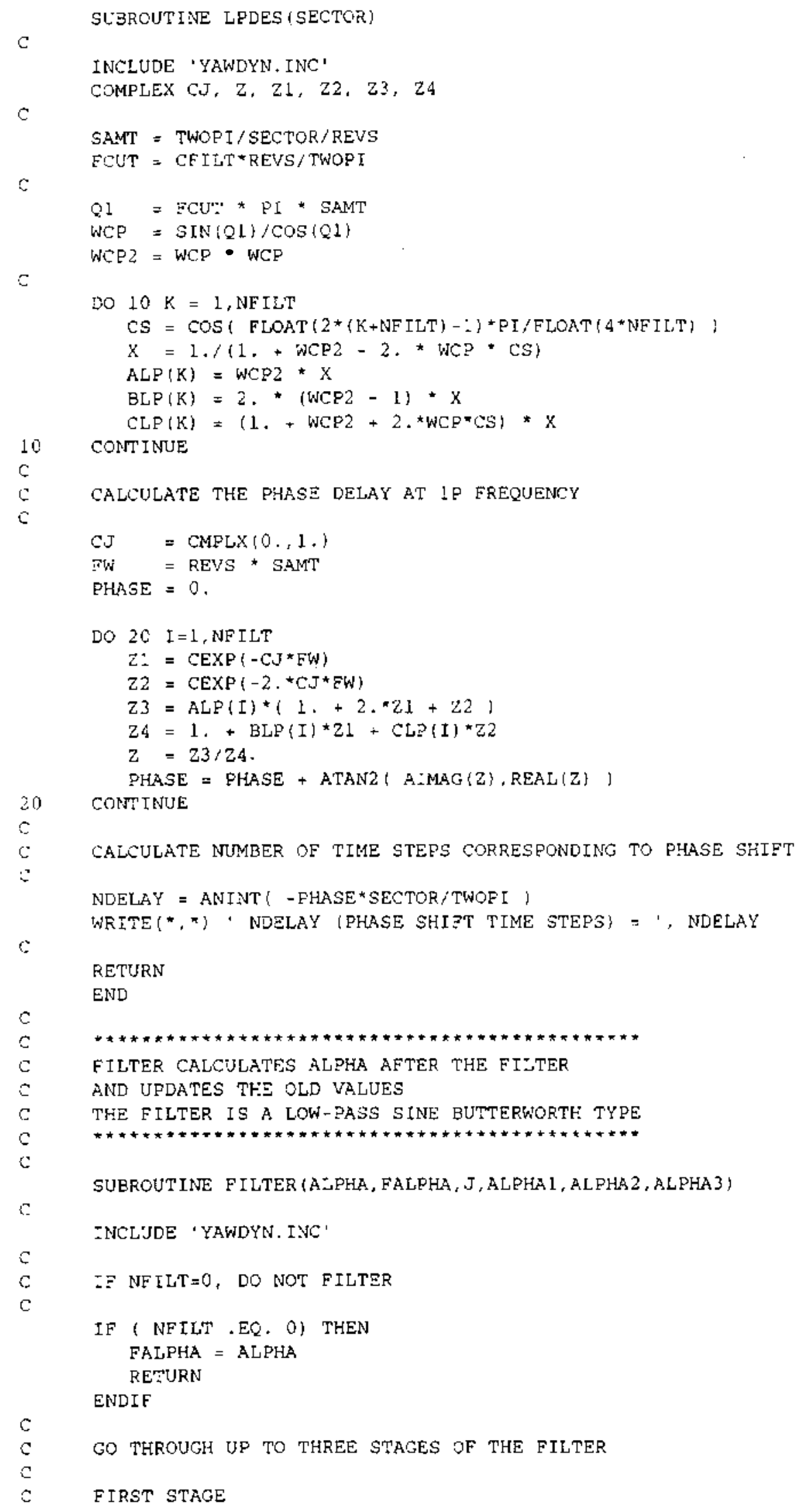




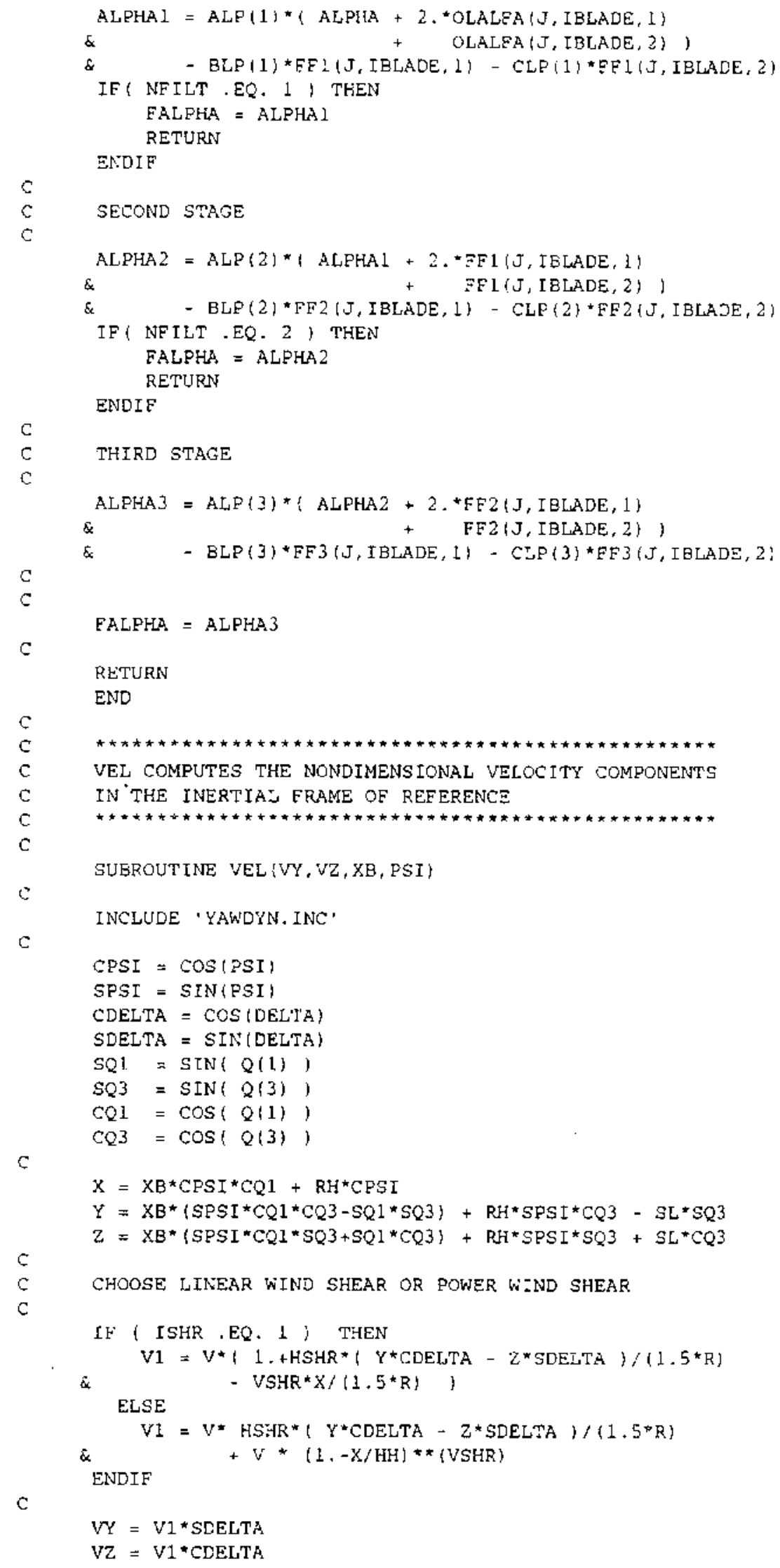


$c$

$\mathrm{C}$

TLRN

END

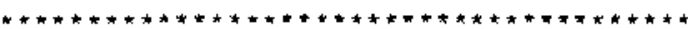

VESD CALCULATE THE VELOCITIES RELATIVE

TO TFE ROTOR DISK

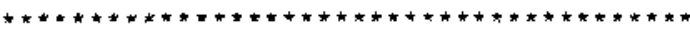

SUBROUTINE VELE (SDEL, CDEL, ANGFLW, NTEST)

INCLUDE 'YAWDYN. INC'

VELOCITIES AT ROTCR HUB IN XYZ COOR.

$V_{Y} 1=V^{*} \operatorname{SIN}(D E L T A)$

$V Z 1=V * \cos (D E L T A)$

CTILT $=\operatorname{COS}(T I L T)$

STILT $=\operatorname{SIN}\{T I L T\}$

$\mathrm{CQ3}=\cos (Q(3))$

$\mathrm{SQ} 3=\operatorname{SIN}(Q(3))$

$\mathrm{VYZ1}=\mathrm{VZ1} 1 \mathrm{CQ} 3-\mathrm{VY} 1 * \mathrm{SQ} 3$

C

VELOCITIES IN ROTOR DISK COOR.

VELI $=V X * C T I L T+V Y Z 1 * S T I L T$

VEL2 $=V Y{ }^{*} \mathrm{CQ} 3+V Z I^{*} \mathrm{SQ}^{3}+\mathrm{SL}^{\star} \mathrm{Q}(4){ }^{*} \mathrm{CTILT} / \mathrm{R}$

VEL3 $=-V{ }^{*}$ STILT + VYZI ${ }^{*}$ CTLLT

$\mathrm{C}$

$\operatorname{VEL}=\operatorname{SQRT}(\operatorname{VEL} 1 * \star 2+V E L 2 * * 2)$

$c$

IF \& VEL , GE, 1,OE-6, THEN

NTEST $=1$

SDEL $=$ VEL $2 / V E L$

CDEL = VEL1/VEL

ANGFLW = ATAN2 $\{$ ABS (VEL $3-A V G I N F L)$, VEL 1 ELSE

ENDIF

NTEST $=0$

C

RETURN

END

VNMOD APPLIES THE SKEWED WAKE CORRECTION

TO THE NORMAL VELOCITY COMPONENT.

TO THE NORAL VELOCITY COMPONENT.

SUBROUT INE VNMOD (VN, XB, A, AXY, PSI, SDEL, CDEL, ANGFLW)

INCLUDE ' YAWDYN. INC

SANG $=$ SIN (ANGFLW $)$ 
C

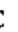

$=V N *\left(1--^{-A X Y}\right\} /(1-A)$

RETURN

END

$\mathrm{C}$

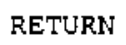


C

FUNCTION GETCD - 5 TNTERPOLATION ROUTINE POR AIRFOIL CD

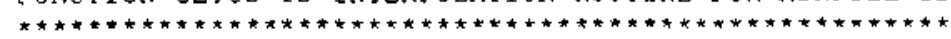

FUNCTION GETCD (ANG)

$c$

$c$

INCLUDE 'YAWDYN. INC'

GETCD $=-999$.

IF( ANG .LT. AD(1) .OR. ANG .GT, AD (NDRAG) ) RETURN

$\mathrm{c}$

CALE LOCATE (AD, NDRAG, ANG, N)

$P=(A N G-A D(N)) /(A D(N+1)-A D(N))$

$\operatorname{GETCD}=C D(N)+P *(C D(N+1)-C D(N)\}$

RETURN

END

$\mathrm{C}$

$+* * *+4$

SUBROUTINE LOCATE FINDS PCSITION IN AIRFOIL TABLE

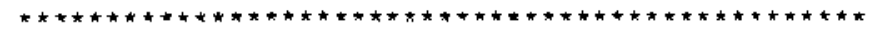

SEE 'NUMERICAI RECIPES' BY PRESS, ET AL, PAGE 89.

SIMPLIFIED TO WORK ONLY WITH MONOTONLCALLY INCREASING

VALUES OF XX IN THE TABLE. ACH $10 / 88$.

VARIABLES :

$X X$ = ARRAY (ANGLES OF ATTACK IN TABLE)

$\mathrm{N}$ = NIMBER OF ENTRIES IN THE TABLE

$\mathrm{X}$ = GIVEN VALUE OF ANGLE OF ATTACK

$J \quad=$ INDEX FOSITION OF $X \quad$ IN TABLE

$J$ IS SUCH THAT $X$ IS BETWEEN XXIJ) AND $X X(J+1)$

SUBROUTINE LCCATE (XX,N, X, J)

DIMENSION $\mathrm{XX}\{\mathrm{N}\}$

$J L=0$

$\mathrm{JU}=\mathrm{N}+1$

$10 \mathrm{IF}(\mathrm{JU}-J \mathrm{U}) . \mathrm{GT}, 1)$ THEN

$J M=(J U+J L) / 2$

IF( X .GT. XX (JM) , THEN

$J L=M$

ELSE

ENDIF

$\mathrm{JU}=\pi \mathrm{M}$

GO TO 10

ENDIF

$J=J L$

RETURN

END

c

c

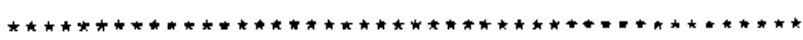

FUNCTION FPL CALCULATES PLATE LIFT COEFFICIENT

USING THE VITERNA METHOD

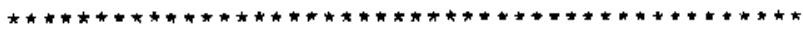

EUNCTION FPL (ANG)

INCLUDE 'YAWDYN. INC'

C 
A1 $=\operatorname{CDMAX} / 2$.

$S S I=S I N(A L(N L I F T))$

$S C O=\cos (A L(N L I F T))$

$\mathrm{A})=(\mathrm{CL}(\mathrm{NLIFT})-\mathrm{CDMAX} * \mathrm{SSI} * \mathrm{SCO}) * \mathrm{SSI} / \mathrm{SCO} / \mathrm{SCO}$

FIND PROPER QUADRANT AND ASSIGN CL THE ASSIGNNENTS ARE AS EOLLOWS:

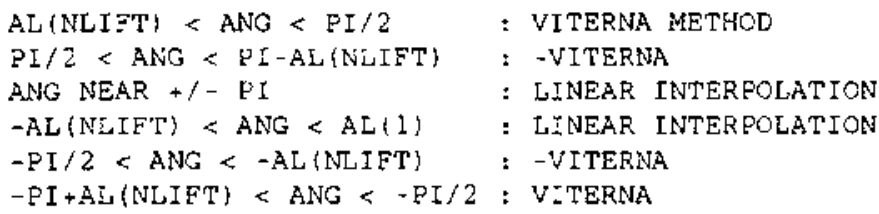

IF( ANG .GT. AL(NLIET) .AND. ANG .LE. PIBY2) THEN SANG = STN (ANG) COSANG $=\cos ($ ANG) 
c

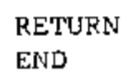




\section{Listing of the YawDyn.Inc include file}

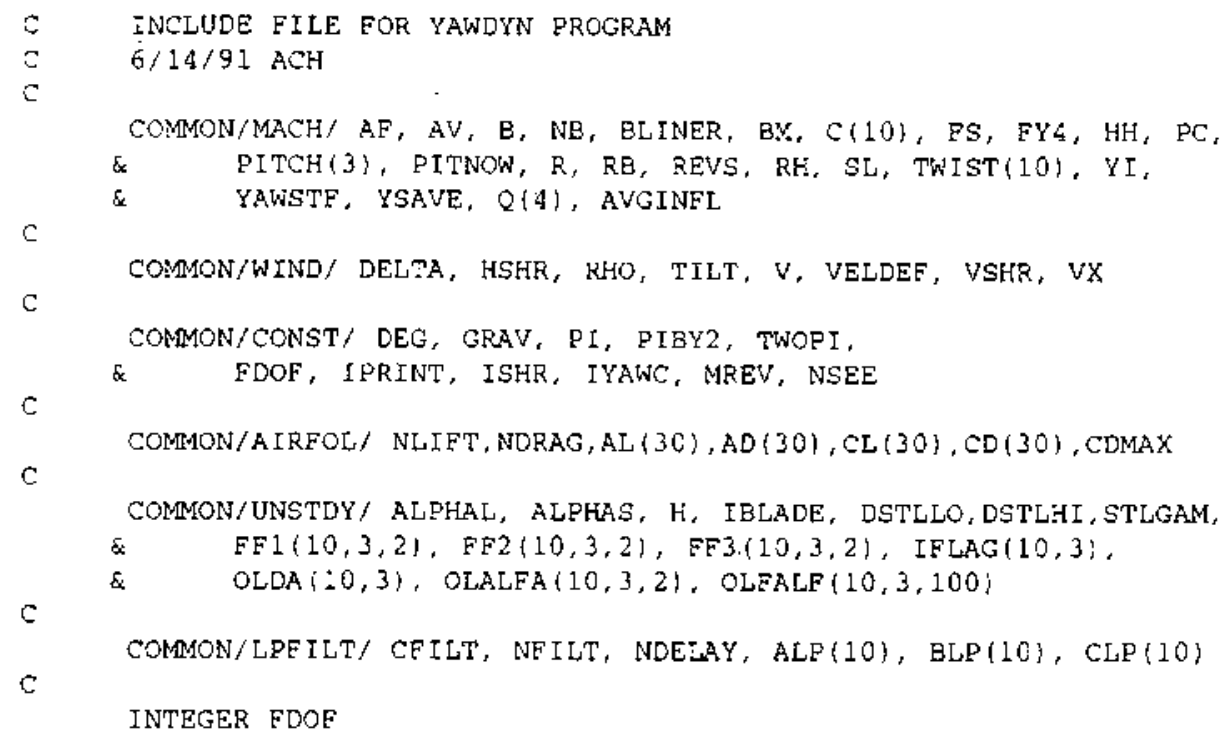




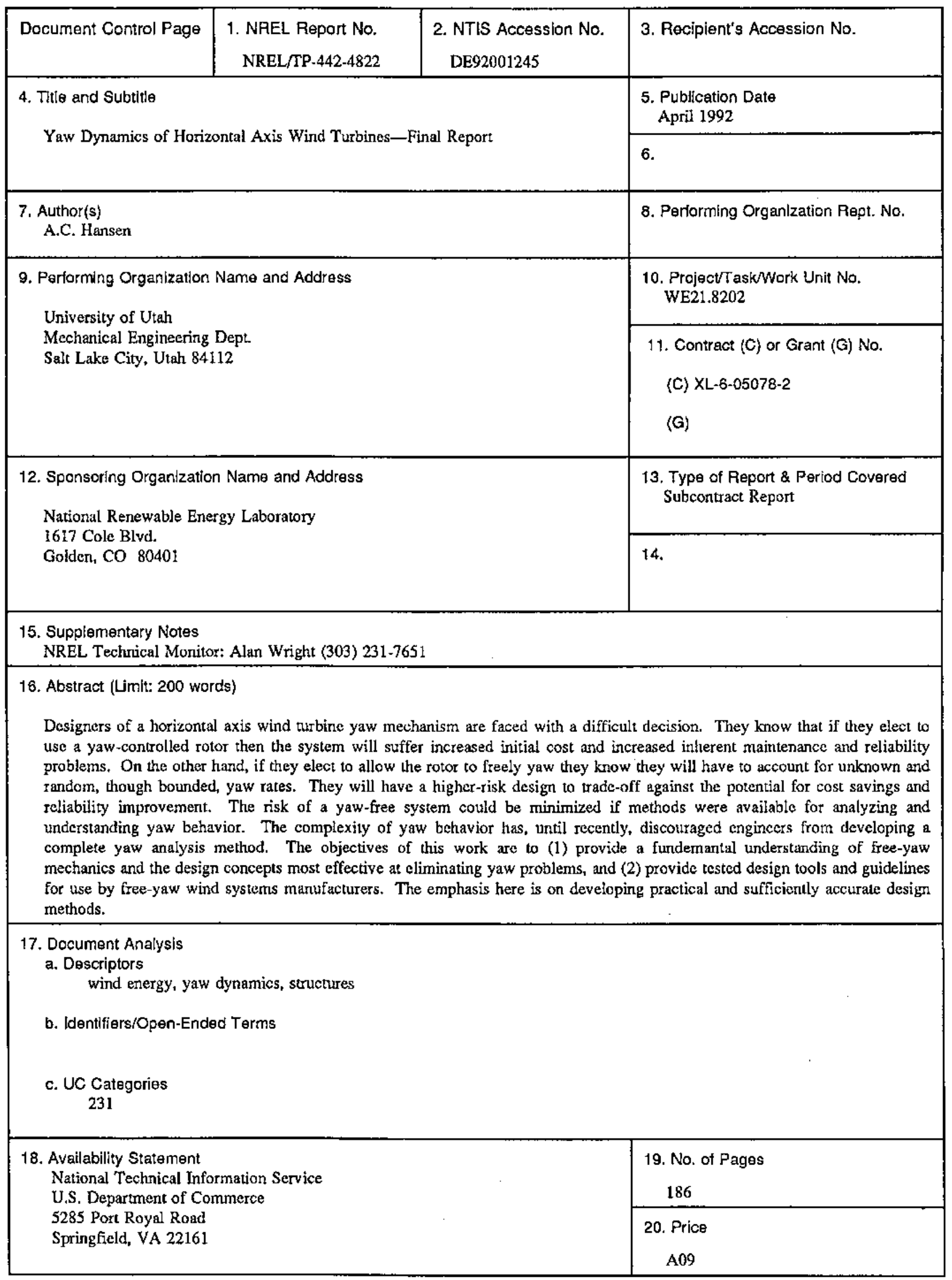

Form No. 0069E (6-30-87) 